\title{
Geostatistical three-dimensional modeling of the subsurface unconsolidated materials in the Göttingen area:
}

The transitional-probability Markov chain versus traditional indicator methods for modeling the geotechnical categories in a test site

\section{Dissertation}

Submitted as a partial fulfilment for obtaining the "Philosophy doctorate (Ph.D.)" degree in graduate program of the Applied Geology department, Faculty of Geosciences and Geography, Georg-August-University School of Science (GAUSS), Göttingen

Submitted by (author):

Ranjineh Khojasteh, Enayatollah

Born in Tehran, Iran 


\title{
Geostatistical three-dimensional modeling of the subsurface unconsolidated materials in the Göttingen area:
}

The transitional-probability Markov chain versus traditional indicator methods for modeling the geotechnical categories in a test site

\author{
Dissertation \\ zur Erlangung des mathematisch-naturwissenschaftlichen Doktorgrades \\ " Philosophy doctorate (Ph.D.)" \\ der Georg-August-Universität Göttingen \\ im Promotionsprogramm Angewandte Geoliogie, Geowissenschaften / Geographie \\ der Georg-August University School of Science (GAUSS) \\ vorgelegt von \\ Ranjineh Khojasteh, Enayatollah
}

aus (geboren): Tehran, Iran

Göttingen , Frühling 2013 
Betreuungsausschuss: Prof. Dr. Ing. Thomas Ptak-Fix, Prof. Dr. Martin Sauter, Angewandte Geologie, Geowissenschaftliches Zentrum der Universität Göttingen

Mitglieder der Prüfungskommission: Prof. Dr. Ing. Thomas Ptak-Fix, Prof. Dr. Martin Sauter, Angewandte Geologie, Geowissenschaftliches Zentrum der Universität Göttingen

Referent: $\quad$ Prof. Dr. Ing. Thomas Ptak-Fix

Korreferent: $\quad$ Prof. Dr. Martin Sauter

ggf. 2. Korreferent:

weitere Mitglieder der Prüfungskommission:

1- - PD. Dr. Eckehard Holzbecher;

2- - Dr. Raimon Tolosana Delgado;

3- - Dr. Pavel Propastin;

4- - J/Prof. Dr. Sonja Philipp.

Tag der mündlichen Prüfung: $\quad$ 27.Juni. 2013 


\section{Dedicated to}

My beloved parents and brothers

....and all who devoted their best belongings and loves to my movement, success, and prosperity

who all greatly share all my achievements

, and to my beautiful land 


\section{Abstract:}

Having a plenty of geotechnical records and measurements in Göttingen area, a subsurface three-dimensional model of the unconsolidated sediment classes was required. To avoid the repetition of the long expressions, from this point on, these unconsolidated materials which vary from the loose sediments to the hard rocks has been termed as "soil", "category", "soil class" or "soil category". These sediments which are intermediate between the hard bed-rock and loose sediments (soils) were categorized based on the geotechnical norms of the DIN 18196.

In this study, the aim was to evaluate the capabilities of the application of geostatistical estimation and simulation methods in modeling the subsurface heterogeneities, especially about the geotechnical soil classes. Such a heterogeneity modeling is a crucial step in a variety of applications such as geotechnics, mining, petroleum engineering, hydrogeology, and so on. For an accurate modeling of the essential continuous parameters, such as the ore grades, porosity, permeability, and hydraulic conductivity of a porous medium, the precise delineation of the facies or soil category boundaries prior to any modeling step is necessary. The focus of this study is on a three-dimensional modeling and delineation of the unconsolidated materials of the subsurface using the geostatistical methods. The applied geostatistical methods here consisted of the pixelbased conventional and transition-probability Markov chain-based geostatistical methods. After a general statistical evaluation of different parameters, the presence and absence of each category along the sampling boreholes was coded by new parameters called indicators. The indicator of a category in a sampling point is one (1) when the category exists and zero (0) when it is absent. Some intermediate states can also be found. For instance, the indicator of a two categories can be assigned to 0.5 when both the categories probably exist at that location but it is unsure which one exactly presents at that location. Moreover, to increase the stationarity characteristic of the indicator variables, the initial coordinates were transformed into a new system proportional to the top and bottom of the modeled layer as a first modeling step. In the new space, to conduct the conventional geostatistical modeling, the indicator variograms were calculated and modeled for each category in a variety of directions. In this text, for easier reference to the semivariograms, the term variogram has been applied instead. 
Using the indicator kriging, the probability of the occurrence of each category at each modeling node was estimated. Based on the estimated probabilities of the existence of each soil category from the previous stage, the most probable category was assigned to each modeling point then. Moreover, the employed indicator variogram models and indicator kriging estimation parameters were validated and improved. The application of a less number of samples were also tested and suggested for similar cases with a comparable precision in the results. To better reflect the fine variations of the categories, the geostatistical simulation methods were applied, evaluated, and compared together. The employed simulation methods consisted of the sequential indicator simulation (SISIM) and the transition probability Markov chain (TP/MC). The conducted study here suggested that the TP/MC method could generate satisfactory results especially compared to those of the SISIM method. Some reasons were also brought and discussed for the inefficiency of the other facies modeling alternatives for this application (and similar cases).

Some attempts for improving the TP/MC method were also conducted and a number of results and suggestions for further researches were summarized here. Based on the achieved results, the application of the TP/MC methods was advised for the similar problems. Besides, some simulation selection, tests, and assessment frameworks were proposed for analogous applications. In addition, some instructions for future studies were made.

The proposed framework and possibly the improved version of it could be further completed by creating a guided computer code that would contain all of the proposed steps.

The results of this study and probably its follow-up surveys could be of an essential importance in a variety of important applications such as geotechnics, hydrogeology, mining, and hydrocarbon reservoirs. 


\section{Zusammenfassung:}

Das Ziel der vorliegenden Arbeit war die Erstellung eines dreidimensionalen Untergrundmodells der Region Göttingen basierend auf einer geotechnischen Klassifikation der unkosolidierten Sedimente. Die untersuchten Materialen reichen von Lockersedimenten bis hin zu Festgesteinen, werden jedoch in der vorliegenden Arbeit als Boden, Bodenklassen bzw. Bodenkategorien bezeichnet.

Diese Studie evaluiert verschiedene Möglichkeiten durch geostatistische Methoden und Simulationen heterogene Untergründe zu erfassen. Derartige Modellierungen stellen ein fundamentales Hilfswerkzeug u.a. in der Geotechnik, im Bergbau, der Ölprospektion sowie in der Hydrogeologie dar.

Eine detaillierte Modellierung der benötigten kontinuierlichen Parameter wie z. B. der Porosität, der Permeabilität oder hydraulischen Leitfähigkeit des Untergrundes setzt eine exakte Bestimmung der Grenzen von Fazies- und Bodenkategorien voraus. Der Fokus dieser Arbeit liegt auf der dreidimensionalen Modellierung von Lockergesteinen und deren Klassifikation basierend auf entsprechend geostatistisch ermittelten Kennwerten. Als Methoden wurden konventionelle, pixelbasierende sowie übergangswahrscheinlichkei tsbasierende Markov-Ketten Modelle verwendet.

Nach einer generellen statistischen Auswertung der Parameter wird das Vorhandensein bzw. Fehlen einer Bodenkategorie entlang der Bohrlöcher durch Indikatorparameter beschrieben. Der Indikator einer Kategorie eines Probepunkts ist eins wenn die Kategorie vorhanden ist bzw. null wenn sie nicht vorhanden ist. Zwischenstadien können ebenfalls definiert werden. Beispielsweise wird ein Wert von 0.5 definiert falls zwei Kategorien vorhanden sind, der genauen Anteil jedoch nicht näher bekannt ist. Um die stationären Eigenschaften der Indikatorvariablen zu verbessern, werden die initialen Koordinaten in ein neues System, proportional zur Ober- bzw. Unterseite der entsprechenden Modellschicht, transformiert. Im neuen Koordinatenraum werden die entsprechenden Indikatorvariogramme für jede Kategorie für verschiedene Raumrichtungen berechnet. Semi-Variogramme werden in dieser Arbeit, zur besseren Übersicht, ebenfalls als Variogramme bezeichnet. 
Durch ein Indikatorkriging wird die Wahrscheinlichkeit jeder Kategorie an einem Modellknoten berechnet. Basierend auf den berechneten Wahrscheinlichkeiten für die Existenz einer Modellkategorie im vorherigen Schritt wird die wahrscheinlichste Kategorie dem Knoten zugeordnet. Die verwendeten Indikator-Variogramm Modelle und Indikatorkriging Parameter wurden validiert und optimiert. Die Reduktion der Modellknoten und die Auswirkung auf die Präzision des Modells wurden ebenfalls untersucht. Um kleinskalige Variationen der Kategorien auflösen zu können, wurden die entwickelten Methoden angewendet und verglichen. Als Simulationsmethoden wurden "Sequential Indicator Simulation" (SISIM) und der "Transition Probability Markov Chain" (TP/MC) verwendet. Die durchgeführten Studien zeigen, dass die TP/MC Methode generell gute Ergebnisse liefert, insbesondere im Vergleich zur SISIM Methode. Vergleichend werden alternative Methoden für ähnlichen Fragestellungen evaluiert und deren Ineffizienz aufgezeigt.

Eine Verbesserung der TP/MC Methoden wird ebenfalls beschrieben und mit Ergebnissen belegt, sowie weitere Vorschläge zur Modifikation der Methoden gegeben. Basierend auf den Ergebnissen wird zur Anwendung der Methode für ähnliche Fragestellungen geraten. Hierfür werden Simulationsauswahl, Tests und Bewertungsysteme vorgeschlagen sowie weitere Studienschwerpunkte beleuchtet.

Eine computergestützte Nutzung des Verfahrens, die alle Simulationsschritte umfasst, könnte zukünftig entwickelt werden um die Effizienz zu erhöhen.

Die Ergebnisse dieser Studie und nachfolgende Untersuchungen könnten für eine Vielzahl von Fragestellungen im Bergbau, der Erdölindustrie, Geotechnik und Hydrogeologie von Bedeutung sein. 


\section{Table of contents}

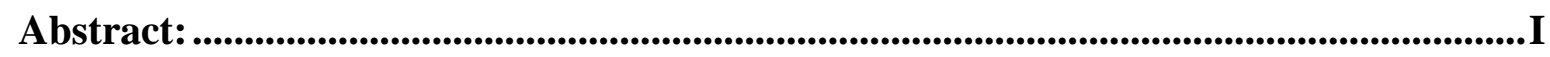

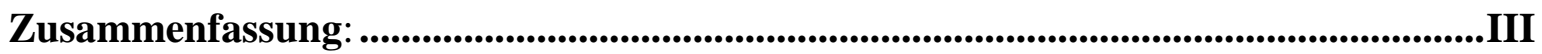

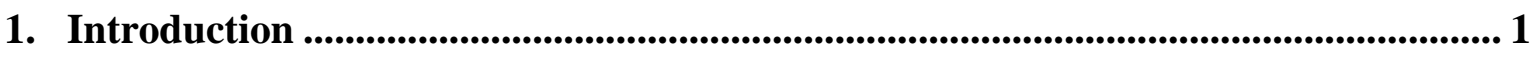

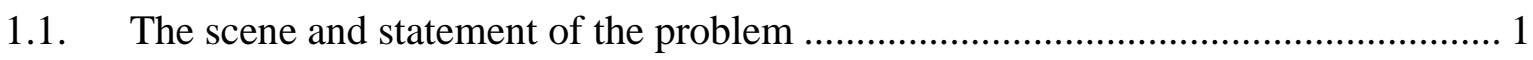

1.2. An introduction to modeling and its applications in earth science problems ............ 2

1.2.1. Definition and the categorization of the models.............................................. 2

1.2.2. The importance and necessity of the three-dimensional modeling for

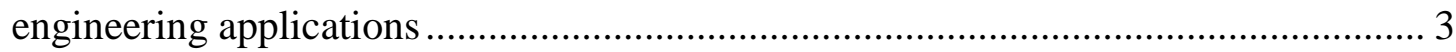

1.3. An overview to the three-dimensional subsurface modeling project in Göttingen area 7

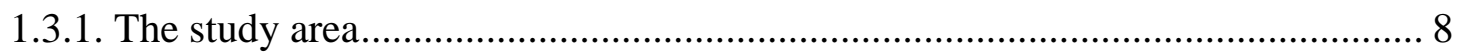

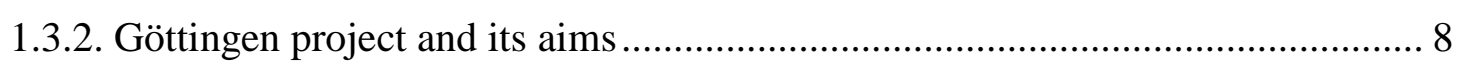

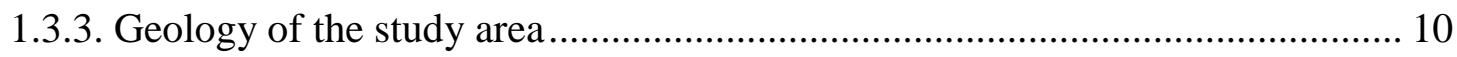

1.3.4. Sampling and samples evaluations ............................................................... 12

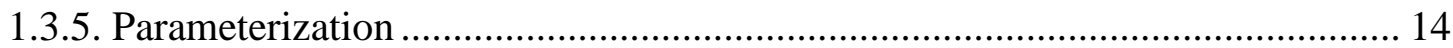

1.4. An introduction to the geostatistical modeling methods (a comparison of different

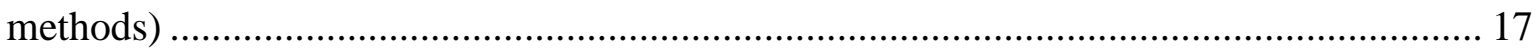

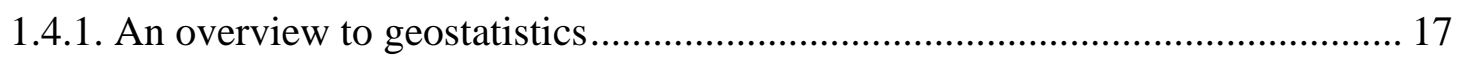

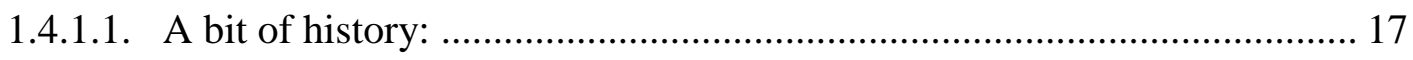

1.4.1.2. The estimation problem and geostatistics:............................................. 17

1.4.2. Some basic concepts in geostatistics ........................................................... 19

1.4.3. Kriging and geostatistical simulation basics ................................................ 31

1.4.4. A comparison of some geostatistical modeling (estimation/simulation)

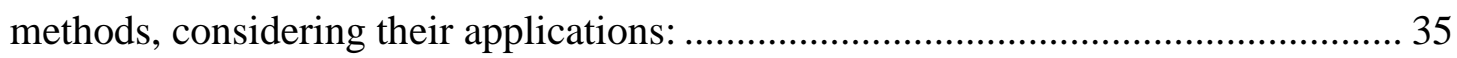

1.4.5. Summary and highlights of the compared methods ....................................... 36

\section{The general workflow of the geostatistical subsurface modeling in Göttingen test}

\section{site45}

2.1. The investigation site and data 47

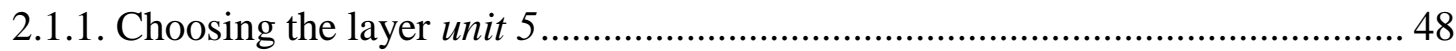


2.1.2. Grid transformation 49

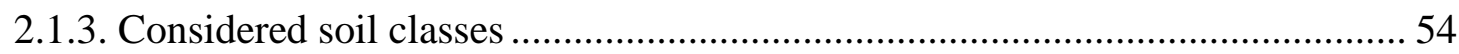

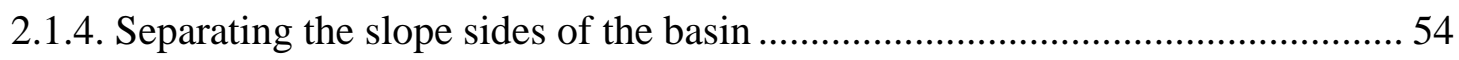

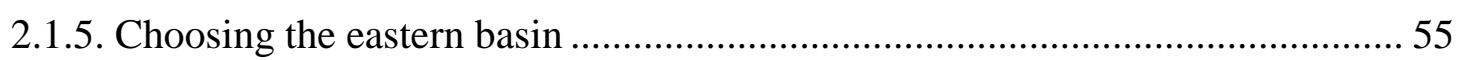

2.1.6. Summary statistics of the data-sets in Göttingen test site ................................ 56

3. Indicator kriging (IK) analysis in the Göttingen test site .................................... 62

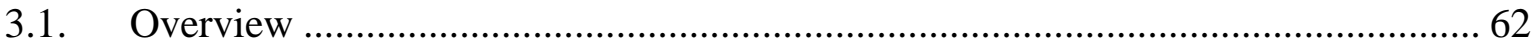

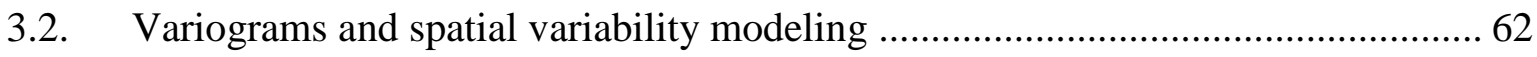

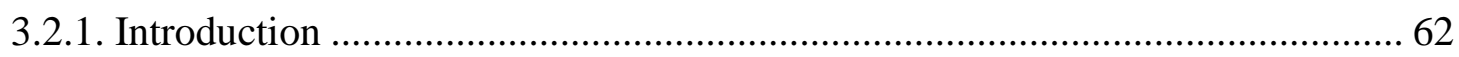

3.2.2. The place of interpretations in variogram modeling ..................................... 66

3.2.3. The validation of the indicator variogram models......................................... 68

3.3. The indicator kriging (IK) analyses for the Göttingen test site ............................. 73

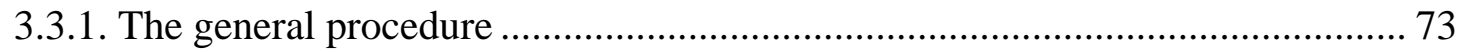

3.3.2. The effect of using a less number of samples on the estimations..................... 78

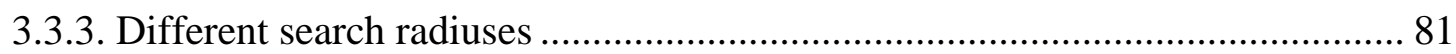

3.4. Models of the soil categories from the indicator kriging …................................ 81

\section{Sequential indicator simulation (SISIM) of the geotechnical soil classes in}

Göttingen test site ........................................................................................................................................... 85

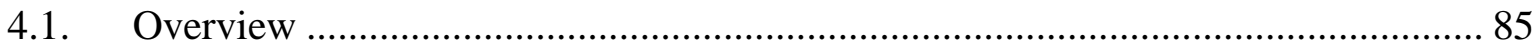

4.2. SISIM for the geotechnical soil classes of the Göttingen project ........................... 86

4.3. Checking the realizations of the sequential indicator simulation (SISIM) method

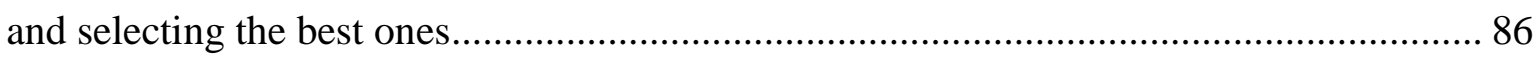

4.3.1. Criteria for checking the goodness of the simulation results.......................... 87

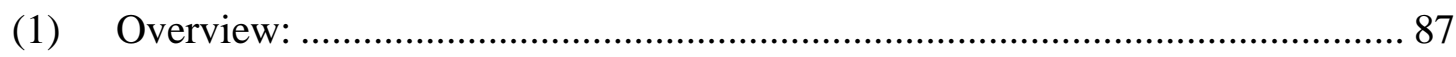

(2) Honoring the input data and histogram reproduction for the realizations of the

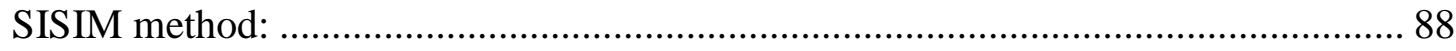

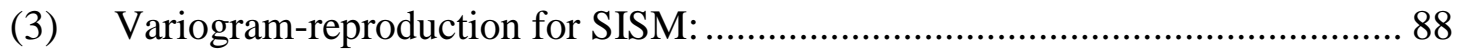

4.3.2. The transition-probabilities- reproduction of the SISIM realizations: .............. 95

4.4. Three-dimensional sections of the selected SISIM realization: ............................ 97

\section{Transition-probability Markov chain (TP/MC) method for modeling subsurface} heterogeneities in Göttingen pilot area, layer 5 .................................................................. 103

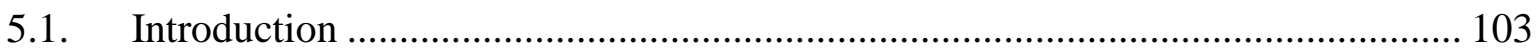




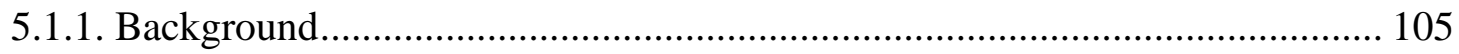

5.1.2. Transition probability-based indicator geostatistics ..................................... 106

5.1.3. The modeling stages in the TP/MC technique............................................. 113

5.1.4. Markov chain models of transition-probabilities.......................................... 115

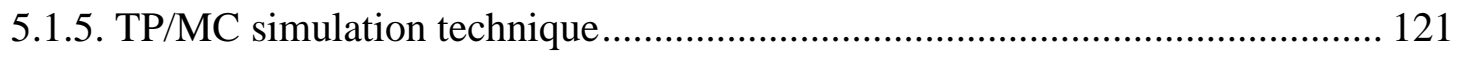

5.2. Transition-probability Markov chain (TP/MC) geostatistical modeling of

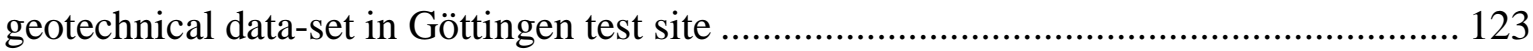

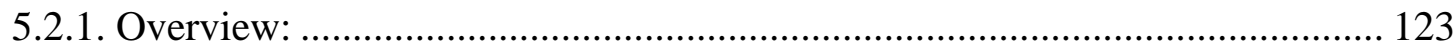

5.2.2. Some points about using post-quenching phase in TSIM program of the T-

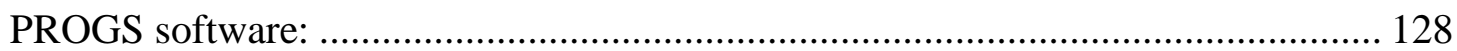

5.3. Evaluating the TP/MC simulation results and their underlying models................ 128

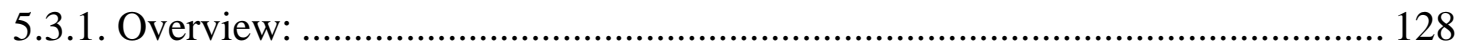

5.3.2. Honoring the input (conditioning) data values at their locations or data

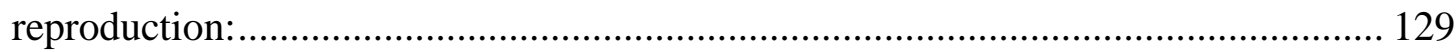

5.3.3. Histogram- (or proportions-) reproduction:................................................. 133

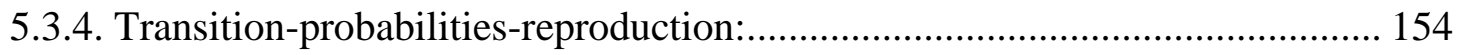

5.3.5. Variograms-reproduction of the transition-probability Markov chain

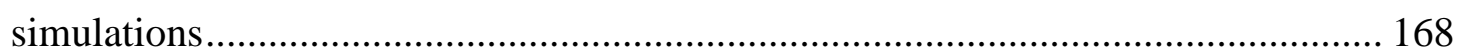

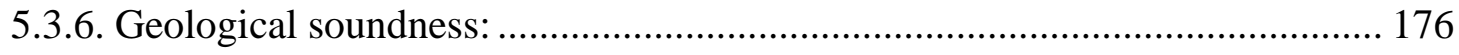

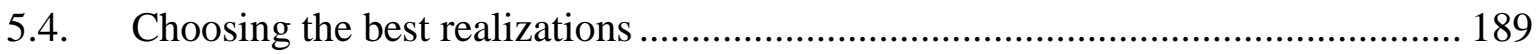

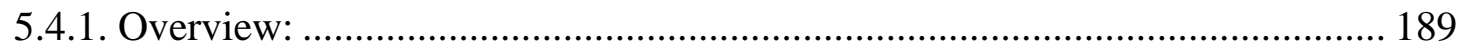

5.5. Some attempts to improve the TSIM algorithm ................................................ 191

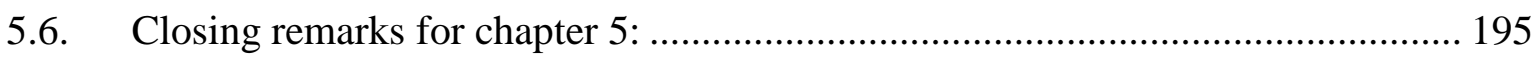

6. Comparison of different geostatistical simulation methods based on their results196

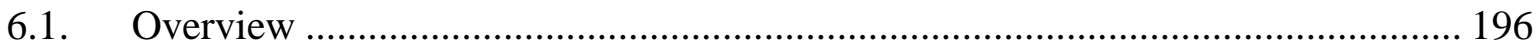

6.2. Evaluations of the geostatistical simulation methods........................................ 196

6.2.1. Based on the (geo-) statistical factors............................................................. 196

6.2.2. Evaluations based on geological acceptability: ........................................... 201

6.2.3. Evaluations based on the speed of the algorithms and the ease of their

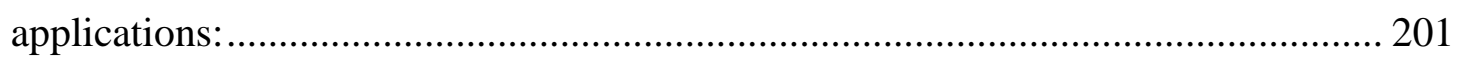

6.3. A number of practical points on modeling steps in this research ......................... 202

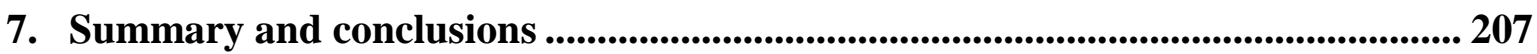


7.1. Synopsis

7.2. Evaluations of the geostatistical realizations and simulation methods

7.3. Some suggestions, comparisons, and conclusions inferred from this study.......... 214

7.4. Suggestions for further research

\section{List of figures}

Figure 1.1 Location map of the study area (translated from Wagner et al. 2007). 8

Figure 1.2 Schematic geological section through the Leine-Valley, Göttingen (Wagner et al. 2007, p. 4, modified from Meischner 2002).

Figure 1.3 Geological section of the study zone, with WE length of 6900 and 20 times vertical exaggeration (Nix et al. 2009, p. 1).

Figure 1.4 Exploded view of the central section of the 3D subsoil model (view from southeast, tilted, extension E/W: $6900 \mathrm{~m}$, extension N/S: $1700 \mathrm{~m}$, vertical exaggeration 15x (Nix et al. 2009, p. 1). For Description of the model units, see section 1.3.4.

Figure 1.5 The stationarity of means for a regionalized variable, (A): referring to a stationary mean, (B): to a non-stationary mean with a trend, and (C): non-stationary case (Hattermann 2011, p. 17).

Figure 1.6 An example of the case of the presence of areal trends has been depicted here. In such cases, each well does not capture the full range of variability. In this example, well A faces mostly high values while the low values are observed in well B (from Gringarten and Deutsch 2001, p. 514).

Figure 1.7 Vertical semivariogram with a zonal anisotropy in which the variogram does not reach its expected sill (Deutsch 2002, p. 121).

Figure 1.8 A typical variogram that can be applied in spatial visibilities modeling. The dots show the sample variogram and the solid curve represents the model variogram. The straight thin solid lines project the important variogram parameters of range (a) and sill (c). 
Figure 1.9 Anisotropic variograms; (A) variogram with the same sill and (B) linear variogram with different slopes (Sarma 2009, p. 83).

Figure 1.10 Samples of elliptical anisotropy; (A) main axes follow the direction of the co-ordinate axes and (B) main axes do not follow the direction of the co-ordinate axes (Sarma, 2009 p. 84).

Figure 1.11 An illustration of the subsurface estimation problem in environmental applications. $\mathrm{W}_{1}$ to $\mathrm{W}_{5}$ represent the drilling locations for getting subsurface samples or measurements.

Figure 1.12 The definition of the simulation grid and cell indexes for coupled Markov chain methods in equation (1-26) (Figure adapted from Elfeki and Dekking 2001, p. 574).43

Figure 2.1 General workflow of geostatistical modeling stages of Göttingen test site...... 46

Figure 2.2 Southeast thee-dimensional view of the study area and the drilled boreholes locations applied in this modeling, with 20 times exaggeration in the vertical direction (Wagner et al. 2007).

Figure 2.3 Horizontal sample variograms of the four geotechnical soil categories without vertical (Z) co-ordinate transformation (i.e. in original $\mathrm{Z}$ system), plotted in three directions; red representing the NS direction, blue representing the EW direction, and black representing the Omni-directional variogram. The horizontal solid black line represents the expected sills of each sample variogram.

Figure 2.4 Location map representing the mode clusters (soil classes) of the Pleistocene layer in pilot zone in eastern and western basin of Göttingen soils project area (488 boreholes) excluding too deep samples (from the deep holes). Colors show the mode cluster (most observed) soil class in each borehole.

Figure 2.5 Location map representing the mode clusters (classes) of the Pleistocene layer in pilot zone in Eastern basin of Göttingen soils project area (188 boreholes) excluding too deep samples (from the deep holes). Colors show the mode cluster in each borehole. 52 
Figure 2.6 Various gridding systems for geostatistical modeling in different geological scenarios (Falivene et al. 2007, p. 203).

Figure 2.7 A schematic illustration of solifluction materials, their location, and formation (Solifluction [Solifluction or Frost Creep (example)]. Copyright 2000-2001. Photograph (Image). Index of Teacher, Geology 12, Photos, Belmont Secondary School. Web.)........ 55

Figure 2.8 The weighted histogram of the soil classes derived from the input data after de-clustering

Figure 2.9 Scatter-plot representing the occurrence of soil clusters along the Xcoordinate.

Figure 2.10 Scatter-plot representing the occurrence of soil clusters along the Ycoordinate.

Figure 2.11 Scatter-plot representing the occurrence of soil clusters along the $\mathrm{Z}_{\text {relative }}{ }^{-}$ coordinate.

Figure 2.12 Scatter-plot of the top versus bottom elevation surfaces elevations of the study layer.

Figure 2.13 Scatter-plot of the top elevation versus Pleistocene layer thickness (the study layer).

Figure 2.14 -plot of the bottom elevation versus Pleistocene layer thickness (the study layer).

Figure 3.1 Experimental and model indicator variograms of geotechnical soil classes 1 to 4, in horizontal and vertical directions (left-side and right-side graphs, respectively). Red, purple, green, gray, and blue solid lines in horizontal variograms represent N-S, NE-SW, E-W, SE-NW, and Omni-directional variograms, respectively. In the vertical variograms, red lines represent the sample vertical variograms. The black dashed lines show the model variograms for both vertical and horizontal variograms.

Figure 3.2 Experimental and model indicator variograms of geotechnical soil classes 1 to 4, in horizontal and vertical directions (left-side and right-side graphs, respectively). Blue 
solid lines in horizontal variograms represent Omni-directional variograms. In the vertical variograms, red lines represent the sample vertical variograms. The black solid lines show the model variograms for both vertical and horizontal variograms.

Figure 3.3 Histogram of the indicator kriging (IK) results for the soil class estimations in terms of the proportions of each soil category in estimated model. The class which held the highest probability was assigned to the estimation grid of the model in each estimation point. 75

Figure 3.4 Indicator variograms of the indicator kriging (IK) results for the soil class estimations created by assigning the most probable soil classes to each estimation point. The graphs on the left and right side represent the horizontal and vertical indicator variograms, respectively. Black lines represent the model variograms while the red and blue lines demonstrate the indicator variograms of the estimated model. The red and blue lines on the left side show the indicator variograms of the horizontal estimation model along the NS and EW directions respectively. The red lines on the left graphs represent the vertical variograms of the estimated IK model

Figure 3.5 Sample and model indicator variograms of the soil categories in horizontal (left side graphs) and vertical directions (right side graphs). Sample variograms were calculated by the half of the existing boreholes. Red lines represent the sample variograms and the black lines represent the corresponding model. 79

Figure 3.6 A perspective top view of the IK model including the lowermost surface (floor). The model represents a $2180 \mathrm{~m}$ distance in EW and a $1580 \mathrm{~m}$ distance in NS direction with $15 x$ exaggeration in the vertical direction.

Figure 3.7 A perspective tilted top view of the IK model with fence diagram sections. The model represents a $2180 \mathrm{~m}$ distance in EW and a 1580m distance in NS direction with $15 x$ exaggeration in the vertical direction.

Figure 3.8 A perspective top view of the IK model with fence diagram sections along the basin. The model represents a $2180 \mathrm{~m}$ distance in EW and a $1580 \mathrm{~m}$ distance in NS direction with $15 x$ exaggeration in the vertical direction. 
Figure 3.9 A perspective top-view of the IK model with fence diagram sections along the basin. The model represents a $2180 \mathrm{~m}$ distance in EW and a $1580 \mathrm{~m}$ distance in NS direction with $15 x$ exaggeration in the vertical direction.

Figure 3.10 A top view of the IK model showing the lowermost surface (floor) of the basin model. The model represents a $2180 \mathrm{~m}$ distance in EW and a $1580 \mathrm{~m}$ distance in NS direction with $15 x$ exaggeration in the vertical direction.

Figure 4.1 Checking the variogram-reproduction of realization 37 for SISIM method. Red and blue lines represent the simulation and black lines show the model variograms. In the left side graphs which represent the horizontal variograms, the red lines illustrate the variograms of the simulation in the NS, and the blue lines represent the simulation variogram in EW direction. The right-side graphs show the vertical variograms in which the red lines show the simulation variogram.

Figure 4.2 Checking the sequential indicator simulation variogram reproduction for realization numbers 37 and 61 . Colored lines represent the simulation and black lines show the model variograms. The left side graphs represent the horizontal variograms whereas the right side graphs are the vertical variograms. The red and purple lines in the horizontal variograms show the NS, and the dark and light blue lines in the horizontal variograms represent the EW directions, respectively. Red and purple lines in the vertical variograms show the vertical sample variograms of the realizations 37 and 61, respectively

Figure 4.3 Sequential indicator simulation (SISIM) variograms for 100 realizations (red and blue lines) in horizontal (left graph; red for EW, and blue for NS) and vertical directions (right graphs) versus model variograms (black lines).

Figure 4.4 The transition-probabilities of the SISIM realizations number 37 (points) and 61 (crosses), versus their corresponding Markov chain models for the horizontal direction of the existing soil classes in the study zone. The calculations have been conducted in .... 96

Figure 4.5 The transition-probabilities of the SISIM realizations number 37 (points) and 61 (crosses), versus their corresponding Markov chain models for the horizontal direction of the existing soil classes. 
Figure 4.6 A perspective top view of the realization 37, generated by the SISIM simulation method. The model represents a $2180 \mathrm{~m}$ distance in EW and a $1580 \mathrm{~m}$ distance in NS direction with $15 x$ exaggeration in the vertical direction.

Figure 4.7 A perspective bottom view of the realization 37, generated by the SISIM simulation method. The model represents a $2180 \mathrm{~m}$ distance in EW and a $1580 \mathrm{~m}$ distance in NS direction with $15 x$ exaggeration in the vertical direction.

Figure 4.8 A perspective top side view of the realization 37, generated by the SISIM simulation method. The model represents a $2180 \mathrm{~m}$ distance in EW and a $1580 \mathrm{~m}$ distance in NS direction with $15 x$ exaggeration in the vertical direction.

Figure 4.9 A perspective bottom side view of the realization 37, generated by the SISIM simulation method. The model represents a $2180 \mathrm{~m}$ distance in EW and a $1580 \mathrm{~m}$ distance in NS direction with $15 x$ exaggeration in the vertical direction. 100

Figure 4.10 A top view of the realization 37, generated by the SISIM simulation method. The model represents a $2180 \mathrm{~m}$ distance in EW and a $1580 \mathrm{~m}$ distance in NS direction with $15 x$ exaggeration in the vertical direction.

Figure 4.11 A top view of the realization 37, generated by the SISIM simulation method showing the lowermost surface (floor) of the basin. The model represents a $2180 \mathrm{~m}$ distance in $\mathrm{EW}$ and a $1580 \mathrm{~m}$ distance in NS direction with $15 \mathrm{x}$ exaggeration in the vertical direction.

Figure 4.12 A perspective top side fence-section view of the realization 37, generated by the SISIM simulation method. The model represents a $2180 \mathrm{~m}$ distance in EW and a $1580 \mathrm{~m}$ distance in NS direction with $15 \mathrm{x}$ exaggeration in the vertical direction 101

Figure 4.13 A perspective top side fence-section view of the realization 37 along the SN direction, generated by the SISIM simulation method. The model represents a $2180 \mathrm{~m}$ distance in $\mathrm{EW}$ and a $1580 \mathrm{~m}$ distance in NS direction with $15 \mathrm{x}$ exaggeration in the vertical direction.

Figure 4.14 A perspective top side fence-section view of the realization 37 along the EW direction, generated by the SISIM simulation method. The model represents a $2180 \mathrm{~m}$ 
distance in $\mathrm{EW}$ and a $1580 \mathrm{~m}$ distance in NS direction with $15 \mathrm{x}$ exaggeration in the vertical direction.

Figure 5.1 Transition-probabilities: $\mathrm{t}_{\mathrm{jk}}(\mathrm{h})=\operatorname{Pr}\{\mathrm{k}$ occurs at $\mathrm{x}+\mathrm{h} \mid \mathrm{j}$ occurs at $\mathrm{x}\}$ as a function of separation vector and not the position in a stationary condition. The blue table summarizes the numbers of transition (\#T) from each facies type to another one in matrix format, for the example of the facies column in the logs seen at the left side of the figure, for a specific separation vector of $\Delta h_{z}$. The way of counting the transition for different lag-spacing (red and green arrows here) has been illustrated in the left-side log column (left graph are modified from Carle 1999, p. 8). 104

Figure 5.2 an example of (auto- and cross-) transition-probabilities, and relevant Markov chain models for a two-category facies model of Channel and Not-Channel. Points represent the observed values, solid curves represent the Markov chain models, dashedlines stand for the proportions, and slopes show the estimated slopes from the mean lengths. The graph is taken from Carle and Fogg (1996, p. 459)

Figure 5.3 Transition-probabilities of the four geotechnical soil categories in vertical direction calculated from input data (dots) and their corresponding Markov chain model (solid lines) from transition rates method as a final fine-tuned model.

Figure 5.4 Transition-probabilities of the four geotechnical soil categories in horizontal direction calculated from input data (dots) and their corresponding Markov chain model (solid lines) from transition rates method as a final fine-tuned model.

Figure 5.5 a schematic illustration of how the input data may not be assigned to the simulation grid node: A, the sample exists but it is located outside the simulation grid; B, the existing sample is inside the simulation grid but the value is trimmed; and $\mathrm{C}$, samples exist and are inside the simulation grid but there are more than one samples inside the grid block but only the closest sample is assigned (the graph is taken from Leuangthong (2004, p. 133). 130

Figure 5.6 input data conditioning errors histogram of SISIM simulation method for realization number 20 . 131 
Figure 5.7 Horizontal transition-probabilities among the soil classes for realization 16 generated by TSIM without any quenching step method (dots) and corresponding Markov chain models (solid lines)

Figure 5.8 Vertical transition-probabilities among the soil classes for realization 16 generated by TSIM without any quenching step method (dots) and corresponding Markov chain models (solid lines).

Figure 5.9 Horizontal transition-probabilities among the soil classes for realization 19 generated by TSIM method with a one-step post-quenching phase (dots) and corresponding Markov chain models (solid lines).

Figure 5.10 Vertical transition-probabilities among the soil classes for realization 19 generated by TSIM method with a one-step post-quenching phase (dots) and corresponding Markov chain models (solid lines). 161

Figure 5.11 Horizontal transition-probabilities among the soil classes for realization 12 generated by TSIM method with a two-step post-quenching phase (dots) and corresponding Markov chain models (solid lines). 162

Figure 5.12 Vertical transition-probabilities among the soil classes for realization 12 generated by TSIM method with a two-step post-quenching phase (dots) and corresponding Markov chain models (solid lines). 163

Figure 5.13 Horizontal transition-probabilities among the soil classes for realization 19 generated by TSIM method with a four-step post-quenching phase (dots) and corresponding Markov chain models (solid lines). 164

Figure 5.14 Vertical transition-probabilities among the soil classes for realization 19 generated by TSIM method with a four-step post-quenching phase (dots) and corresponding Markov chain models (solid lines). 165

Figure 5.15 Horizontal transition-probabilities among the soil classes for realization 1 generated by TSIM method with a four-step post-quenching phase (dots), corresponding Markov chain models (solid lines), and realization 37 generated by SISIM method (cross symbols). 166 
Figure 5.16 Vertical transition-probabilities among the soil classes for realization 1 generated by TSIM method with a four-step post-quenching phase (dots), corresponding Markov chain models (solid lines), and realization 37 generated by SISIM method (cross symbols). 167

Figure 5.17 Indicator variograms of all 20 realizations generated by the TSIM simulation method without any quenching steps (colored lines), and their corresponding model variograms (black lines). The graphs on the left side represent the horizontal variograms (red for North-South, and dark blue for East-West directions), and the graphs on the right side represent the vertical variograms.

Figure 5.18 Indicator variograms of all 20 realizations generated by the TSIM simulation method with one post-quenching step (colored lines), and their corresponding model variograms (black lines). The graphs on the left side represent the horizontal variograms (red for North-South, and dark blue for East-West directions), and the graphs on the right side represent the vertical variograms.

Figure 5.19 Indicator variograms of all 20 realizations generated by the TSIM simulation method with two post-quenching steps (colored lines), and their corresponding model variograms (black lines). The graphs on the left side represent the horizontal variograms (red for North-South, and dark blue for East-West directions), and the graphs on the right side represent the vertical variograms.

Figure 5.20 A perspective top view of the realization 12, generated by the TSIM with two post-quenching steps simulation method. The model represents a $2180 \mathrm{~m}$ distance in EW and a $1580 \mathrm{~m}$ distance in NS direction with 15x exaggeration in the vertical direction. 180

Figure 5.21 A perspective side view of the realization 12, generated by the TSIM with two post-quenching steps simulation method. The model represents a $2180 \mathrm{~m}$ distance in EW and a $1580 \mathrm{~m}$ distance in NS directions with $15 \mathrm{x}$ exaggeration in the vertical direction.

Figure 5.22 A perspective side view of the realization 12, generated by the TSIM with two post-quenching steps method. The model represents a 2180m distance in EW and a $1580 \mathrm{~m}$ distance in NS directions with $15 \mathrm{x}$ exaggeration in the vertical direction. 181 
Figure 5.23 A perspective bottom view of the realization 12, generated by the TSIM with two post-quenching steps simulation method. The model represents a $2180 \mathrm{~m}$ distance in EW and a $1580 \mathrm{~m}$ distance in NS directions with $15 \mathrm{x}$ exaggeration in the vertical direction. 182

Figure 5.24 A perspective bottom view of the realization 12, generated by the TSIM with two post-quenching steps simulation method. The model represents a $2180 \mathrm{~m}$ distance in EW and a $1580 \mathrm{~m}$ distance in NS directions with $15 \mathrm{x}$ exaggeration in the vertical direction.

Figure 5.25 A perspective bottom view of the realization 12, generated by the TSIM with two post-quenching steps simulation method. The vertical slice 21 has been depicted. The model represents a $2180 \mathrm{~m}$ distance in EW and a $1580 \mathrm{~m}$ distance in NS directions with $15 x$ exaggeration in the vertical direction. 183

Figure 5.26 A perspective bottom view of the realization 12, generated by the TSIM with two post-quenching steps simulation method. The vertical slice 14 has been depicted. The model represents a $2180 \mathrm{~m}$ distance in EW and a $1580 \mathrm{~m}$ distance in NS directions with $15 \mathrm{x}$ exaggeration in the vertical direction.

Figure 5.27 A perspective bottom view of the realization 12, generated by the TSIM with two post-quenching steps simulation method. The vertical slice 7 has been depicted. The model represents a $2180 \mathrm{~m}$ distance in EW and a $1580 \mathrm{~m}$ distance in NS directions with $15 x$ exaggeration in the vertical direction.

Figure 5.28 A perspective bottom view of the realization 12, generated by the TSIM with two post-quenching steps simulation method. The vertical slice 2 has been depicted. The model represents a $2180 \mathrm{~m}$ distance in EW and a $1580 \mathrm{~m}$ distance in NS directions with $15 x$ exaggeration in the vertical direction. 184

Figure 5.29 A perspective fence-model bottom side view of the realization 12, generated by the TSIM with two post-quenching steps simulation method. The vertical slice 2 has been depicted. The model represents a $2180 \mathrm{~m}$ distance in EW and a $1580 \mathrm{~m}$ distance in NS directions with $15 x$ exaggeration in the vertical direction. 185 
Figure 5.30 a perspective fence-model bottom view of the realization 12, generated by the TSIM with two post-quenching steps simulation method. The vertical slice 2 has been depicted. The model represents a $2180 \mathrm{~m}$ distance in EW and a $1580 \mathrm{~m}$ distance in NS directions with $15 x$ exaggeration in the vertical direction.

Figure 5.31 a perspective fence-model top view of the realization 12 , generated by the TSIM with two post-quenching steps simulation method. The vertical slice 2 has been depicted. The model represents a $2180 \mathrm{~m}$ distance in EW and a $1580 \mathrm{~m}$ distance in NS directions with $15 x$ exaggeration in the vertical direction. 186

Figure 5.32 A perspective fence section bottom side view along the NS direction of the realization 12 , generated by the TSIM with two post-quenching steps simulation method. The vertical slice 2 has been depicted. The model represents a 2180m distance in EW and a $1580 \mathrm{~m}$ distance in NS directions with $15 \mathrm{x}$ exaggeration in the vertical direction 186

Figure 5.33 Another perspective fence section bottom view along the NS direction of the realization 12 , generated by the TSIM with two post-quenching steps simulation method. The vertical slice 2 has been depicted. The model represents a $2180 \mathrm{~m}$ distance in EW and a $1580 \mathrm{~m}$ distance in NS directions with $15 x$ exaggeration in the vertical direction.

Figure 5.34 A top view of the realization 16, generated by the TSIM without any quenching step simulation method. The model represents a $2180 \mathrm{~m}$ distance in EW and a $1580 \mathrm{~m}$ distance in NS directions with 15x exaggeration in the vertical direction.

Figure 5.35 A top view of the realization 19, generated by the TSIM with a quenching step simulation method. The model represents a $2180 \mathrm{~m}$ distance in EW and a $1580 \mathrm{~m}$ distance in NS directions with $15 x$ exaggeration in the vertical direction. 188

Figure 5.36 A top view of the realization 12, generated by the TSIM with two quenching step simulation method. The model represents a $2180 \mathrm{~m}$ distance in EW and a $1580 \mathrm{~m}$ distance in NS directions with $15 x$ exaggeration in the vertical direction. 188

Figure 6.1 Vertical indicator variograms of the generated realizations by the SISIM method of the 20-realization run (left-side red lines) and 100-realization run (right side red lines) and their corresponding models (black lines). 203 
Figure 6.2 Horizontal indicator variograms of the generated realizations by the SISIM method of the 20-realization run (left-side red lines) and 100-realization run (right side red lines) and their corresponding models (black lines).

\section{List of tables}

Table 1.1 Geotechnical unconsolidated sediments classification scheme for sedimants without organic components (Wagner, 2009).

Table 1.2 Geotechnical unconsolidated sediments classification scheme for sedimants with organic components (Wagner, 2009). 16

Table 1.3 Practical summary and comparison of some geostatistical methods based on main framework and the reasons of using them.

Table 1.4 Practical summary and comparison of some geostatistical methods based on the advantages of their applications pros and cons, researchers, where they have been applied and software.

Table 2.1 Summary statistics of the main two-dimensional parameters.

Table 2.2 Representative frequencies and proportions of the soil classes based on the observations in the boreholes with modifications using declustering and considering the representing volumes of each sample after grid transformation.

Table 3.1 Summary of the cross-validation of the indicator variograms, point-by-point and well-by-well indicator kriging (IK). 72

Table 3.2 Summary of the jackknifing of the estimation models.

Table 3.3 Frequencies, proportions, and percentages of the estimated soil classes by the IK method and assigning the most probable class to each estimation grid of the IK estimation model. 
Table 5.1 The table represents a summary of honoring (conditioning on input data) for various geostatistical simulation methods

Table 5.2 Comparison of selected realizations produced with different simulation methods for proportions reproduction using two different suggested histogram reproduction test methods

Table 5.3 Summary of chi-square statistics for different simulation methods applied in this study

Table 5.4 Illustration of the calculations frameworks of the chi-square test of the homogeneity of proportions, and deviation-rate, for the realization number 37 of the SISIM geostatistical simulation method. 154 


\section{Introduction}

\subsection{The scene and statement of the problem}

When a project engineer or technical manager is planning and assessing the future of a geotechnical site, mineral deposit, a hydrocarbon reservoir, or an aquifer, it is tremendously essential for him/her to identify the subsurface conditions thoroughly and as precisely as possible prior to any technical and practical decision. Though, only a minor portion of the total volume from the study zone is usually known having the limited available samples, while the rest parts are totally undetermined. However, the expert should assess and estimate the characteristics of the major unknown points of the model as well.

Suppose that the planning for a huge structure like a dam or a power plant is required. The geotechnical and hydro-geological characteristics of the underlying layers and materials of the foundation should be fully characterized for a proper engineering arrangement for these surveys. In the prediction of the fluid flows in rocks or sediments, either in the petroleum engineering, hydrogeology problems, geotechnical applications, or mining activities, a precise characterization of the porous media and their heterogeneities is undoubtedly a central issue. Another example could be the plan for the exploitation of an ore deposit for a mining project. The ore veins or layers, the gangues, the hydraulic characteristics of the porous media, the weakness surfaces, etc., are key parameters to be determined before deciding about the future and the plan of the mining activities. In all of the mentioned examples, the required characteristics are bounded in some geological limits and borders such as the boundaries of the layers or other geological bodies. Most of the required continuous parameters are rather consistent and similar inside the mentioned borders. Therefore, a precise characterization of the geological boundaries is the first and most important step in every geosciences modeling practice. However, due to the technical and economical limitations, compounded with the geological complexity and difficult access to the subsurface, this practice is considerably challenging.

In most of the cases, it is only feasible to get samples from the subsurface by means of drilling or other sorts of diggings. At times, some surveys can be performed to achieve indirect information from the subsurface. For instance, geophysical measurements can be made to map the underground physical characteristics and relate them to the geological 
features. Though, these measurements are not capable of representing the requested geological characteristics directly while they may contain a considerable amount of uncertainty. These were just a few examples of the difficulties to survey and model the subsurface. Hence, the central question here is how to estimate the required parameters and evaluate the probable underlying uncertainties having a limited set of data and information. Geostatistical methods make possible and simplify the integration of different sources of information, estimation of unknowns, and assessment of the uncertainties contained in the generated model(s) (Caers 2005).

\subsection{An introduction to modeling and its applications in earth science problems}

\subsubsection{Definition and the categorization of the models}

According to the online Schlumberger Oilfield Glossary ${ }^{1}$, a model can be defined as following:

"A representation of a physical property or entity that can be used to make predictions or compare observations with assumptions" is called a model.

Despite its great importance, the subsurface modeling is tremendously a challenging task because of a limited and sometimes indirect access to such a complex heterogeneous space as subsurface. In addition, the heterogeneity and complexity of different characteristics in the subsurface is often too high to be estimated by simplistic estimation methods such as linear interpolation, constant values within polygons, or even by standard well-behaved mathematical functions that easily (Chilès 1999). For instance, the properties of sedimentary bodies vary naturally over the space due to the processes responsible for their generation and evolution. Most of the geological phenomena responsible for the forming subsurface features are so sophisticated that their modeling by most simplistic methods is not sensible.

The most important benefit of making models for subsurface is that the models can act as gateways to integrate expert knowledge from different fields and aggregate data from different sources.

\footnotetext{
${ }^{1}$ Schlumberger Oilfield Glossary, Definition of "Model". Web

<http://www.glossary.oilfield.slb.com/en/Terms/m/model.aspx>. accessed online 2010.
} 
Most of the variations of the continuous attributes in the study zone are confined in discrete boundaries of the lithofacies, different soil types, etc. Therefore, there is an imperative demand to model the limits and borders of geo-bodies (e.g. lithofacies, soiltype bodies, and so on) prior to modeling and prediction of the other continuous characteristics and parameters. Another challenge could be that, these models should be constructed having the restricted information sources. Geostatistical methods provide various tools for consistent and precise modeling the complex subsurface heterogeneity as well as to evaluate the modeling uncertainty (Ranjineh Khojasteh 2002; Hengel 2007, pp. 13-14; Noppe 1994).

Models can be categorized into different groups based on different criteria, for example; deterministic versus stochastic, structure-imitating versus process imitating, forward versus inverse models, and object-based versus grid-based models (Farmer 2005; Falivene et al. 2007).

Deterministic models yield unique results for a given input because of the lack of randomness in the model whereas the stochastic models generate a set of probable results for the same input due to having random deviations (Falivene et al. 2007, p. 204; "Deterministic Model." BusinessDictionary.com accessed 2012).

Structure-imitating models simulate the patterns without paying attention to the processes responsible to their creation whereas the process-imitating models focus on the processes that create these patterns.

Forward models determine the output given the input while in the inverse models, the unknown input is determined having outputs (Falivene et al. 2007; Farmer 2005).

In object-based (Boolean) models, objects (with predefined geometries) are replaced in an extensive common background whereas in the grid-based models, the attributes are assigned to the grids or pixels (or voxels when the pixels have volume) (Falivene et al. 2007, p. 206).

\subsubsection{The importance and necessity of the three-dimensional modeling for engineering applications}

The conventional mapping methods were mainly based on manually drawing the facies and parameter boundaries (by interpretations or simple interpolations) in two-dimensional slices and connecting the boundaries to each other among different slices to get the final three-dimensional images (Deutsch 2002, p. 154).Such manually drawn modeling 
methods do not closely and analytically take the existing three-dimensional data structure into account. In addition, the conventional modeling approaches lack a clear and consistent modeling criterion (Falivene 2006, p. 49).

Nowadays, more advanced three-dimensional data acquisition tools, fast and powerful computers, computational techniques and software, and more powerful modeling techniques became available. Hence, the three-dimensional modeling with interactive powerful and criteria-based modeling tools adapted with the available problems can be applied. Such methods can better support the integration of all available data, expert knowledge, and known mathematical tools and rules to produce a more precise and realistic representations of subsurface (Deutsch 2002, p. 154; Falivene 2006, p. 49).

With the existence of more three-dimensional data sources such as well-bore data and three-dimensional seismic measurements as well as more improved modeling methods, the use of three-dimensional models are expanding. Such models provide better data integration and accuracy.

Some highlights of the importance of the three-dimensional models in the geotechnical and other applications can be summarized in the following points:

\section{a. Geotechnical modeling and foundations:}

The foundation of a structure such as a dam, a bridge, a building, railroad, etc., transmits the loads from the structures to the earth. After estimating the location and the amount of these loads, the geotechnical engineer should devise a plan to explore the subsurface soil types and bedrock characteristics as well as the geological features for evaluating the capacity of bearing the mentioned loads and the involved hazards and risks "Geotechnical Engineering." Wikipedia., 2012). Therefore, locating and determining the weak and bearing layers and their extent, geometry, and characteristics are the first critical steps in locating and designing the structures. It should again be emphasized that the mechanical, geotechnical, and hydrogeological characteristics of the underlying materials of the structures are considerably consistent and similar within the same geological and geotechnical categories. For example, a layer mainly consisting of coarse sediments can represent higher permeability that can fall in a specific geotechnical or geological category. Obviously, layers with similar conditions that fall in the same geotechnical category will show similar properties. Therefore, the delineation of these geo-bodies and the classifications of their geotechnical or geological categories are the most important steps before modeling their continuous characteristics. 


\section{b. Hydrogeology and groundwater aspects in geo-engineering problems:}

The layers located beneath a structure or their foundations which contain high hydraulicconductivity zones with enough thicknesses, can let higher water flows below and around the foundation and endanger the foundations. On the other hand, the layers with fairly low hydraulic conductivity zones can act as barriers that may prevent the foundation failures. Furthermore, the underground flows are highly dependent on the hydrogeological characteristics of the mentioned layers as well as their extent, thickness, geometry, and distributions (Marinoni 2003, p. 45). Probable swelling and shirking, or liquefaction phenomena of the layers beneath the structures or the seepage zones under the dams are among the other examples for the risks related to the hydrogeological and geological characteristics of the structure sites. Moreover, for the hydrogeological applications, the characteristics of the layers should also be determined. Therefore, to characterize the layer types and their texture is the first crucial step in hydrogeological/hydrological and their relevant geotechnical surveys. These examples highlight the importance of the threedimensional determination of the hydrogeological, geological, and geotechnical characteristics of engineering sites thoroughly and with enough details (Hamilton 2005; Lam et al. 1987).

Geostatistical methods offer a set of clear-cut quantitative tools for three-dimensionally delineation of the geotechnical category zones and estimate the required parameters and evaluate the risks and uncertainties involved in these problems.

\section{c. Plasticity and deformable materials and layers:}

Some materials which can represent a plastic deformation or those are too loose especially when they are thick-enough can be hazardous for the foundations. Evaporative or organic sediments are among the materials that can have such potential problems (Das 2011, pp. 14-14 to 14-22).

\section{d. Hazardous soils and quaternary sediments:}

Regarding the foundations, some characteristics of hazardous soils should be taken seriously. For example soil liquefaction that can cause serious hazards such as landslides or the problems during the earthquakes. The soil grain-size distribution, its composition and geological origin and condition, hydrogeological condition, and its density are among the key factors that can affect the susceptibility of a soil to liquefaction (Johansson, Web. 
14 Nov. 2011; "Liquefaction Potential of Cohesionless Soils Geotechnical Design Procedure.", 2007; Lade, 1998:242). Loose to moderately saturated granular soils with weak drainages are more prone to liquefaction. Silty sands or sands and gravels capped or containing seams of impermeable sediments are examples of such soils. In the existence of a loading, especially cyclic undrained loading like earthquake, the volume of the loose sands tend to shrink, which causes a raise in their porewater pressure and hence a decrease in shear strength, i.e. reduction in effective stress that can lead to a liquefaction. The most vulnerable deposits to liquefaction are young sands and silts with particles sized similarly (Holocene-age, well-sorted deposited sediments within the last 10000 years), in beds with thickness of some meters which are saturated with water. Such deposits usually occur along riverbeds, beaches, dunes, and accumulation zones of windblown silt (loess) and sand have been mounted up. Glacial sediments may contain substantial amount of the sediments like quick clay (in Pleistocene epoch) which can cause serious damages such as landslides. The mentioned points highlight the significance of the investigations and explorations of the texture, structure, combination, and the geometry of young (e.g. Holocene and Pleistocene) granular soils such as silty sands, sand and gravels, and clay bodies ("Soil Liquefaction." Wikipedia, 16 May 2012. Web. 21 May 2012).

The Leine river valley and its underlying sediments which contain riverbed and young sediments, especially considering its sediments composition, therefore deserve closer and more careful attention and investigations for the potential engineering risks. Regarding the fact that the Pleistocene structural layers of the Leine valley sediments represent a high variation in the types of the geotechnical and geological soil classes, the main focus of this study was on the Pleistocene sedimentary zone.

Some sediments in the layers beneath the project sites that probably contain special minerals with the deformation characteristics like swelling or shrinking in contact with water, such as sorts of clay minerals, can cause deformations in the underlying layers of the foundations and lead to serious hazards. All of the cited characteristics, which are reflected in the geotechnical and geological categorization of the sediments, and the interactions with the surrounding environment, should be taken into account in the engineering applications.

Considering the points mentioned above and various undiscussed reasons, a precise capable of uncertainty assessments three-dimensional subsurface model is a tremendously valuable and even indispensable tool for decision-making about locating, designing, and building the structures. 


\section{e. Urban development plans:}

A precise three-dimensional model of subsurface about the geology, geotechnical characteristics, and hydrogeology of an area, can act as a precious decision-making tool for urban development planning (e.g. Stoter, Jantien E., and Peter Van. Oosterom 2006; De-fu 2009).

\section{f. The distribution and geometry of the weak and bearing layers:}

In addition to the weak and hazardous zones, one also should identify the distribution and the geometry of the bearing layers and their bearing capacity when planning for the feasibility and the required provisions of making structures according to the expected loads and the importance of the structure (Gedeon 1992).

Bearing capacity of a layer is the capacity of a soil to support the loads applied to the ground. In other words, the bearing capacity is the maximum average contact pressure between the foundation and the soil which should not produce shear failure in the soil ("Bearing Capacity." Wikipedia, 05 Apr. 2012. Web. Apr. 2012).

\section{g. Further foreseen applications:}

Several further uses can be considered for the mentioned three-dimensional subsurface model with the defined categorization scheme. Among these possible applications that such a model can have, groundwater, agriculture, geothermics, and so on could be mentioned.

There are still lots of definitions and discussions regarding the mentioned geotechnical concepts that have not been referred here. Essentially, the attempt in the above section was only to highlight the importance of the three-dimensional modeling in geotechnical and hydrogeological investigations.

However, the main focus of this study is to make a three-dimensional geotechnical model of the subsurface by means of the geostatistical estimation and simulation methods.

\subsection{An overview to the three-dimensional subsurface modeling project in Göttingen area}




\subsubsection{The study area}

The study area is located near the city of Göttingen in Lower Saxony province (Niedersachsen), Germany. The center of the Göttingen project study area is located in the Göttingen Leine valley with the elevation of 140 to $150 \mathrm{~m}$ above the sea level and is divided into two nearly similar halves in the middle of the valley. Some individual districts and connected localities in the study area are extending up to $300 \mathrm{~m}$ above the sea level. The study area includes the most of the city of Göttingen (Wagner et al., 2007).

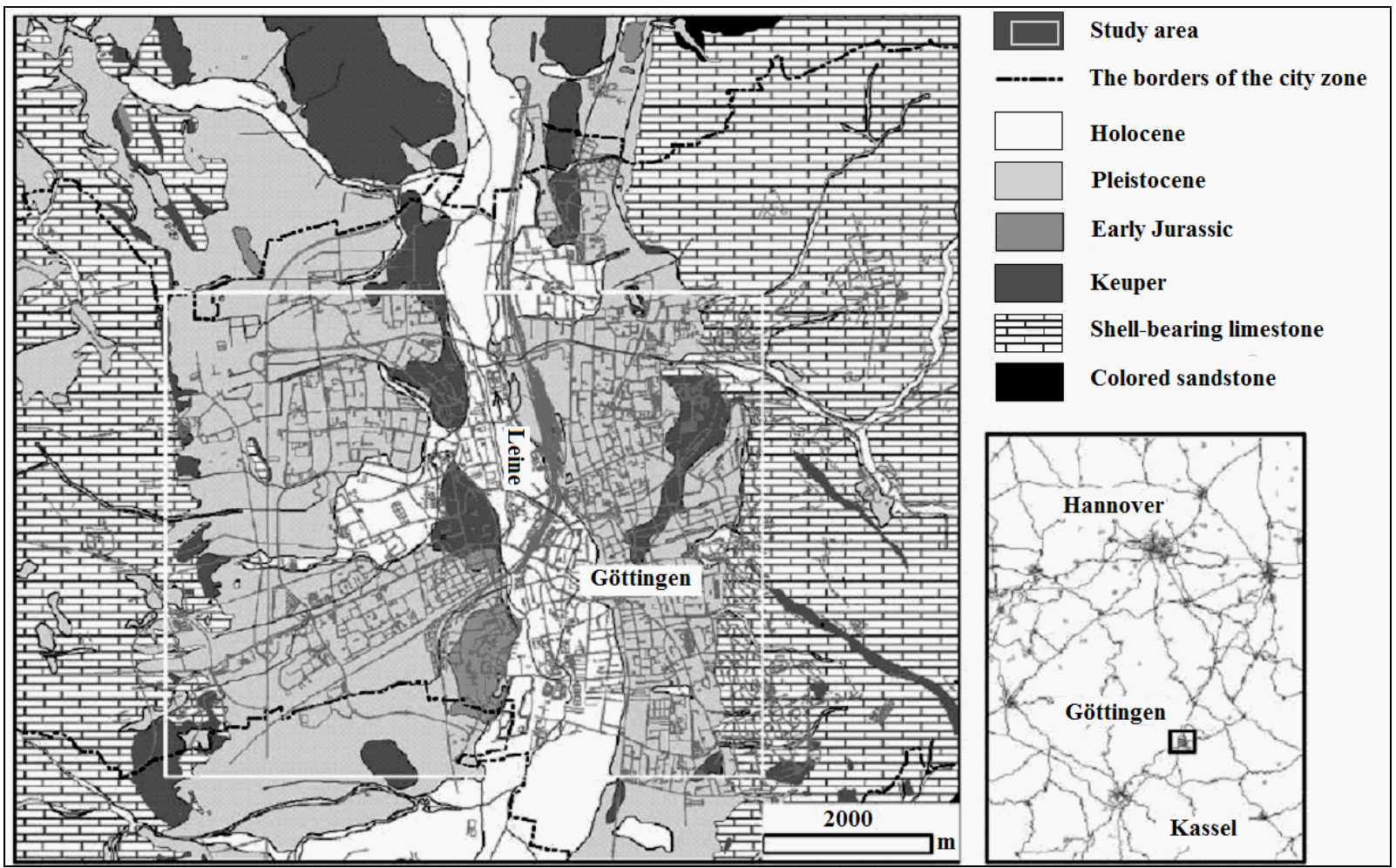

Figure 1.1 Location map of the study area (translated from Wagner et al. 2007).

For geostatistical analysis and modeling purposes which is the focus of this research, a part of the study area was selected as a test site to evaluate the capability and efficiency of the three-dimensional geostatistical modeling of geotechnical types and the comparison among different geostatistical modeling approaches with the emphasis on the transitionprobability Markov chain simulation and optimization methods.

\subsubsection{Göttingen project and its aims}


A high-quality geoscientific subsoil model is in the heart of the geotechnical surveys. Sustainable subsurface development and utilization, necessitates providing comprehensive information about cost-/benefit-/risk-analysis at the planning stage of infrastructure projects or similar (Nix et al. 2009, p.1).

Wagner el al. 2007 (p. 1) has mentioned some aims, challenges, details, and worth of the geotechnical investigations in such areas as following:

"At first view, urban areas do no offer favorable conditions for spacious geoscientific investigations and the three-dimensional visualization of the underground. A multiplicity of anthropogenic and quasi-natural replenishments buries geological outcrops. However, particularly in urban areas geological, hydro-geological and engineering-geological point and areal data are continually collected. This information is usually recorded independently and stored decentrally in variable archiving systems. Just by transferring the variable point and areal data into a 3D model of the urban underground, an overall evaluation becomes possible. Such a 3D model may serve as a database for point and areal data (drillings, profile sections etc.) and provides comprehensive geoscientific planning documents for several topics ranging from site investigation, groundwater exploration, rain water infiltration and flood protection to the estimated use of geothermal energy. In a cooperation project between the Department of Applied Geology of the University of Goettingen $\left(G Z G^{l}\right)$ and the State Authority of Mining, Energy and Geology $\left(L B E G^{2}\right)$, new methods for the design of $3 D$ geological and engineeringgeological models are developed. The application area covers the medium-deep underground of the city of Goettingen within the Leinetal-Graben. This complex geological structure is a result of Mesozoic extensional and compressive movements as well as complex salt tectonics. Quaternary sediments cover large parts of the investigation area with thicknesses varying from $5 \mathrm{~m}$ up to $60 \mathrm{~m}$ in subrosion depressions. Mesozoic rocks crop out at the graben margins. The concepts developed so far, cover the standardization and harmonization of point and areal data as well as the definition of geologically and engineering-geologically relevant modeling units. Within a pilot area, all basic data and $2 D$-sections of the modeling units were merged by Gocad to create a geological 3D model with technically describable basal planes of the modeling units."

Possibilities for data and parameters storage as well as their display are aimed to be presented in the example of the three-dimensional subsurface geotechnical Quaternary model of the city of Göttingen. The basis of three-dimensional model is more than 3,000 wells and geological, geomorphological and pedological maps. The developed 3D building models have illustrated the complex geological structure of the quaternary

\footnotetext{
1 „Geowissenschaftliche Zentrum der Universität Göttingen“= "The geosciences center of the University of Göttingen“.

${ }_{2}^{2}$ „Landesamt für Bergbau, Energie, und Geologie" = "The state office for mines, energy, and geology".
} 
substrate, characterized by Quaternary depositional conditions, salt tectonics and suberosion processes (Nix et al. 2009, p. 1).

\subsubsection{Geology of the study area}

The nearly mirror-constructed geological map (Figure 1.1) clearly shows the distinctive structure of the Leine-valley channel. The valley floor is covered by Quaternary unconsolidated rocks, rising only along the central axes that are hard rock beds. These "central bulges" containing clay, silt, and sand of the Keuper and lower Jurassic rocks are open (Wagner et al. 2007, p. 2). The valley sides are from the carbonate successions of the shell limestone and occasionally recorded from Keuper sandstone-built. Particularly, relevant for the recent development of the Göttingen area is the change of resistant rocks multi evaporative sequences (red, middle-Triasic (Muschelkalk (shell-bearing limestone), Middle Keuper) and lightly orderable rocks in the Mesozoic hard bed rocks (Figure 1.2)). At the first glance, simply structured Leine valley rifts (Figure 1.1) have proven a complex structure, which was formed by several processes (Figure 1.2)).

The three-dimensional model of the subsoil has been designed to represent the complex geological structure of the quaternary underground, shaped by the quaternary deposition circumstances, salt tectonics and subrosion processes (Nix et al. 2009, p. 1).

In Wagner et al. (2007), about the geology of the study area it has been mentioned that the trenches according to Arp et al. (2004) were originated through the extension movements in the upper Jurassic and particularly in the Cretaceous period. In the late Cretaceous period, there was a short compressional phase. The inverted trench structure was greatly disturbed by halo-tectonical movements and a distinct stockwerk tectonics. As it has been mentioned there, this is seen according to Grüger et al. (1994) and Meischner (2002) especially in allochthonous particles in steplike arrangement special ditches and till into the modern era active subrosion sinking (Wagner et al. 2007).

Quaternary unconsolidated sediments cover large areas of the valley floor and go up the slopes. The thickness of the Pleistocene and Holocene sediments range from 60 meters in the subrosion depressions to a few tens of centimeters in the valley sides. Subrosion depressions are underground erosions caused by water inflow leaching, e.g. by 
groundwater flow of the underlying rocks such as salts that can create voids leading to the overburden sinking (Wagner et al. 2007; Stefan et al. 1999-2012. Web. 21 May 2012).

The Pleistocene sequence is made of the glacial river deposits, structured loess, basin sediments and solifluction interglacial muds, spring limestones, and the peat and fossiliferous sediment basin deposits. These sediments are extensively found in the working area of often superficial parts overprinted by soil formation processes (Wagner et al. 2007, p. 3).

In the Holocene, minor river sediments, alluvial, and floodplain of stream loams were formed (Wagner et al. 2007, p. 3).

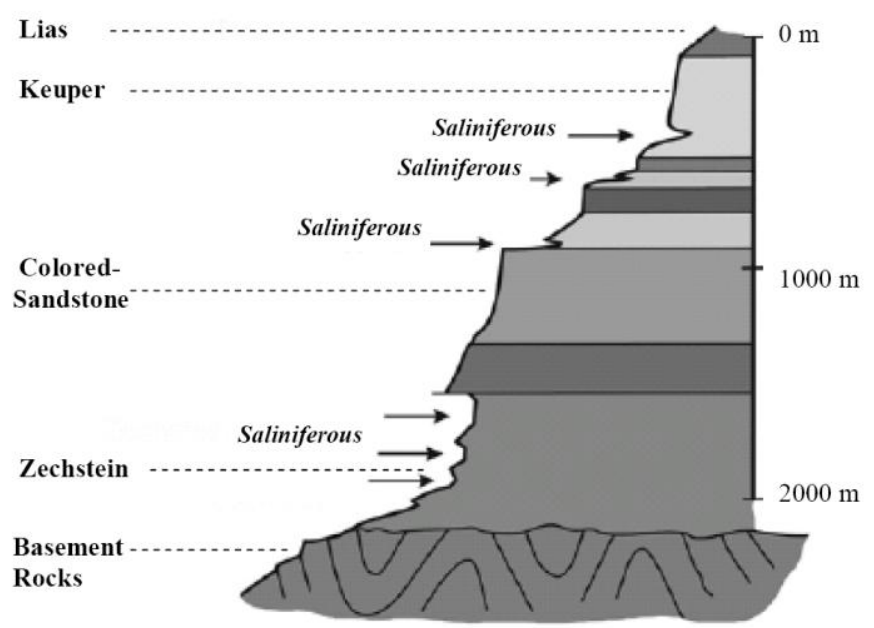

a)

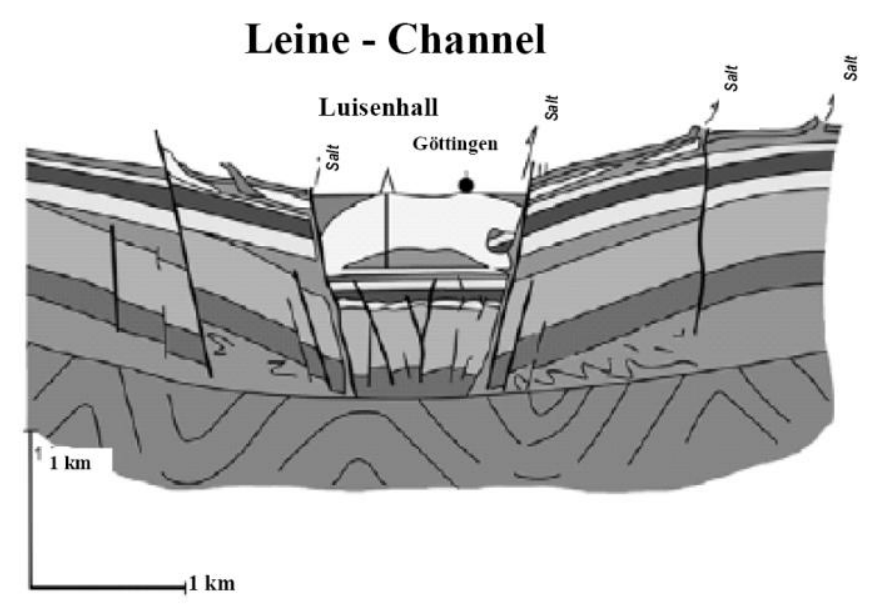

b)

Figure 1.2 Schematic geological section through the Leine-Valley, Göttingen (Wagner et al. 2007, p. 4, modified from Meischner 2002). 


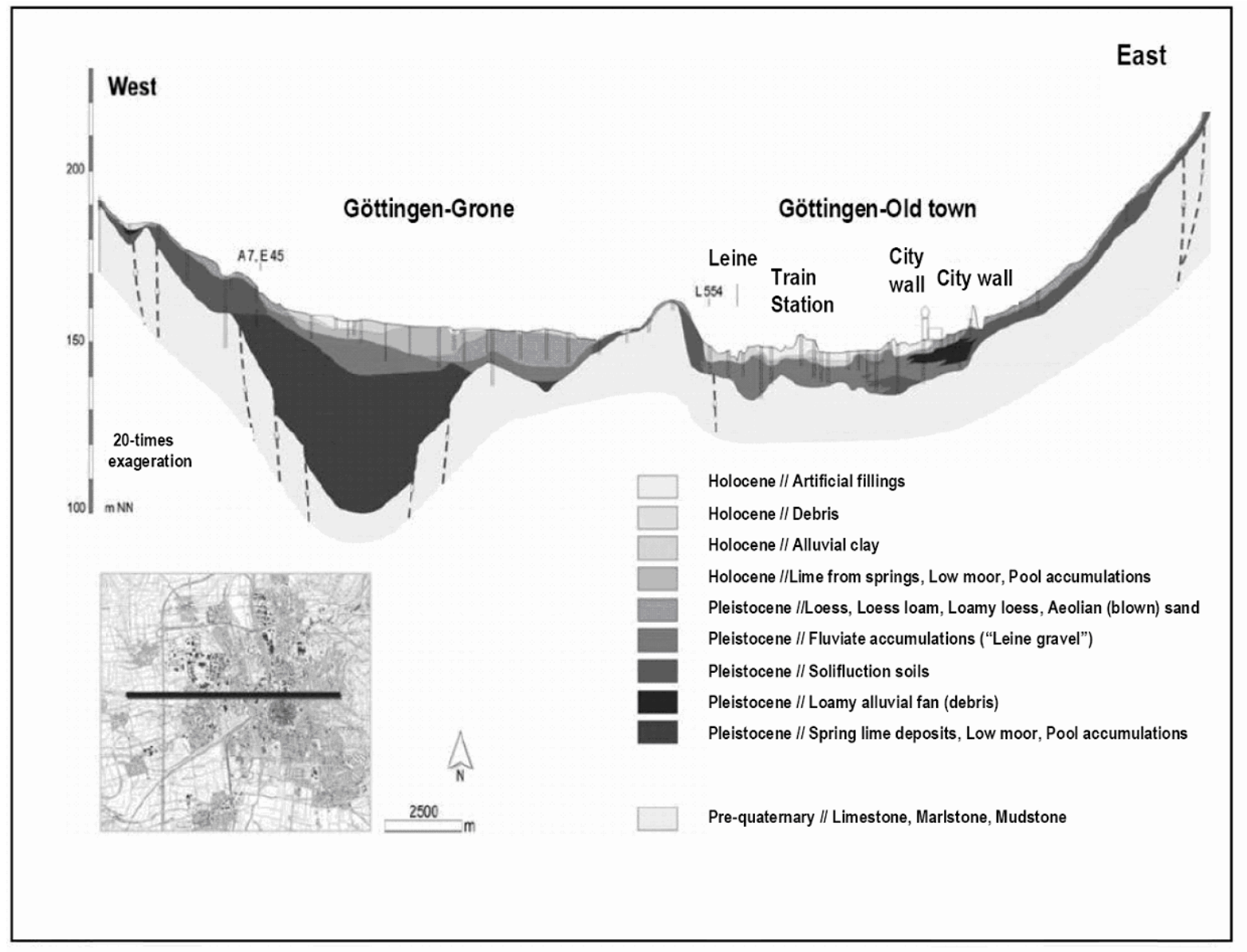

Figure 1.3 Geological section of the study zone, with WE length of 6900 and 20 times vertical exaggeration (Nix et al. 2009, p. 1).

\subsubsection{Sampling and samples evaluations}

The main base for the three-dimensional model of the Göttingen area was 3145 boreholes with different depths, raging from 1 to 244 meters, distributed over the study Göttinegn urban area.

All borehole data were error-checked, homogenized, adjusted for elevation according to the official 5 m-digital elevation model (DGM5), if necessary also digitized in the SEP3 format using the borehole database GeODin ${ }^{1}$ (Wagner et al. 2007). The model integrates a comprehensive set of map data including the geological map of Göttingen (GK25, sheet

\footnotetext{
${ }^{1}$ GeoDin software version 7 (smart data management) "is a modular data management solution enabling you to choose the optimal software solution for your for geological, environmental, geotechnical, monitoring and laboratory projects requirements." by fugro company. ( website: < http://www.geodin.com/software/> ).
} 
4425), 25 geo-engineering maps from diploma ${ }^{1}$ mapping projects, as well as geological, soil type and geomorphological small-scale maps (e.g. Schlie, 1989 and Wunderlich, 1959). In addition more than $30 \mathrm{EW}$ as well as more than $30 \mathrm{NS}$ cross sections was constructed to use the defined layer boundaries as pre-interpreted support during the $3 \mathrm{D}$ modeling.

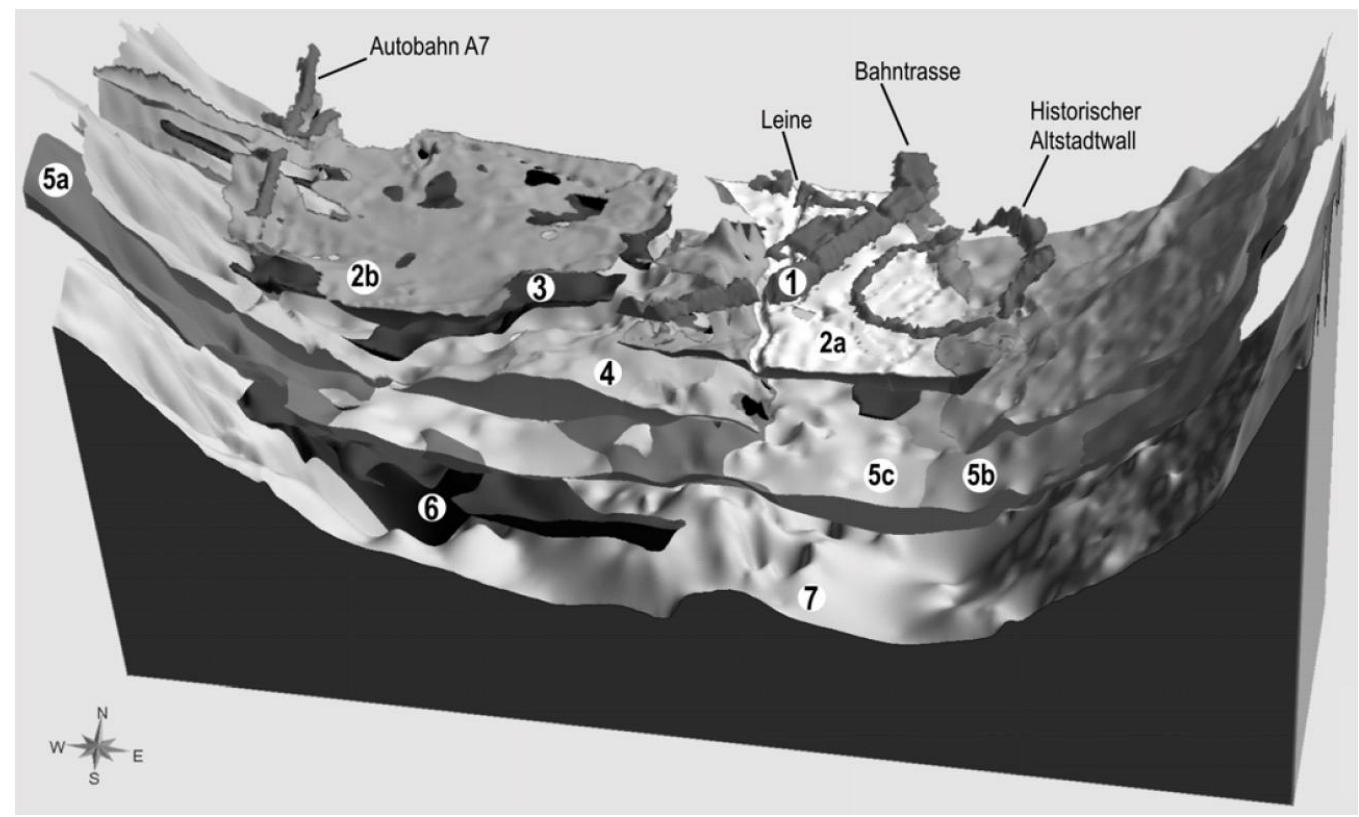

Figure 1.4 Exploded view of the central section of the 3D subsoil model (view from southeast, tilted, extension E/W: $6900 \mathrm{~m}$, extension N/S: $1700 \mathrm{~m}$, vertical exaggeration 15x (Nix et al. 2009, p. 1). For Description of the model units, see section 1.3.4.

Due to the complex spatial pattern of the Quaternary sediments the information had to be translated into a reduced set of structures that would preserve the main and relevant features and guarantee to keep the limited time frame for the modeling within the project. As a result the following model units were defined:

(1) Anthropogenic fillings,

(2a) floodplain sediments and stream loams of younger Holocene,

(2b) younger Holocene debris flows,

(3) Soft Holocene layers (lime from springs, peat, mud),

(4) Pleistocene loess, loamy loes, and flood loess,

(5a) Pleistocene solifluction soils,

(5b) Pleistocene debris,

(5c) Pleistocene river gravels,

\footnotetext{
${ }^{1}$ German diploma academic system is equivalent to M.Sc. in American academic system.
} 
(6) Pleistocene soft layers in subrosion basins,

(7) Pre-quaternary bed rock surface.

Figure 1.4 illustrates the location and distribution of the mentioned layers (Nix et al. 2009).

In this study, the layer 5 was targeted for modeling, because of its geotechnical importance and higher complexity. In addition, the anthropogenic fillings and the superficial sediments were not considered for the geostatistical modeling because they are rather being homogeneous.

\subsubsection{Parameterization}

Because of the uneven and sporadic distribution of measured soil physical or mechanical characteristics, a process was developed to geotechnically describe and estimate the properties of petrographic layers (Fritz et al. 2007). Basis of the classification was six major soil classes out of which four observed classes in the study zone of this dissertation were modeled here (see the summary of classification in Table 1.1 and Table 1.2).

This classification was formed in accordance with the DIN 18196 standard $^{1}$ based on the information from petrography, genesis, and soil layer group descriptions in the equidistant spacing of $0.5 \mathrm{~m}$ above this level are assigned to soil classes (Table 1.1 and Table 1.2). The distribution of soil classes within the three-dimensional sedimentary bodies was determined using a geostatistical indicator kriging (IK), sequential indicator simulation (SISIM), and transition-probability Markov chain simulation approaches using the software WinGslib version 1.5, and TPROGS version 2.1. Here, the probability of the existence of a soil class is estimated in each cell of the estimation model and the uncertainty of the estimation was quantified. Then in each estimation point of the model, the soil class which held the highest probability of occurrence was assigned to the estimation point. Geostatistical estimation was conducted for the distribution of soil classes 1-4 in the model units (5a, b, and c).

\footnotetext{
${ }^{1}$ German standard for geotechnical soil classifications based on grain size range, grain size distribution, plasticity characteristics, organic constituent characteristics, and genesis. For more details refer to Deutsche Institute Für Normung. "Erd Und Grundbau, Bodenklassifikation Für Bauteschniche Zwecke, DIN 18196." Erkundung Und Untersuchung Des Baugrunds: Normen. Vol. 13. Berlin [u.a.: Beuth, 1991. 363-66. Print.
} 
Table 1.1 Geotechnical unconsolidated sediments classification scheme for sedimants without organic components (Wagner, 2009).

\begin{tabular}{|c|c|c|c|c|}
\hline group & $\begin{array}{l}\text { geotech- } \\
\text { nical classes }\end{array}$ & petrology & $\begin{array}{l}\text { main components } \\
\text { (minor } \\
\text { components) }\end{array}$ & facies \\
\hline \multirow{3}{*}{$\begin{array}{l}\text { A } \\
\text { fine-grained } \\
\text { sediments } \\
\end{array}$} & \multirow[t]{3}{*}{3} & clay and silt & $\mathrm{T}, \mathrm{U}, \mathrm{(S})$ & clayey-silty limnic sediment \\
\hline & & pure clay & $\mathrm{T},(\mathrm{U}, \mathrm{S})$ & clayey limnic sediment \\
\hline & & pure silt & $\mathrm{U},(\mathrm{T}, \mathrm{S})$ & silty limnic sediments, loess \\
\hline \multirow{4}{*}{$\begin{array}{l}\text { B } \\
\text { mixed grain-size } \\
\text { sediments } \\
\text { (clay and/or silt } \\
\text { and/or sand) }\end{array}$} & 3 & $\begin{array}{l}\text { clay, silt and } \\
\text { sand }\end{array}$ & $\mathrm{T}, \mathrm{U}, \mathrm{S}$ & $\begin{array}{l}\text { clayey to silty floodplain } \\
\text { deposits, weathered loess }\end{array}$ \\
\hline & 2,3 & clay and sand & $\mathrm{T}, \mathrm{S},(\mathrm{U})$ & weathered loess \\
\hline & 3 & silt and sand & $U, S,(T)$ & loess \\
\hline & 2,3 & sand and silt & $\mathrm{S}, \mathrm{U},(\mathrm{T})$ & aeolian sand, sandy loess \\
\hline \multirow{2}{*}{$\begin{array}{l}\text { C } \\
\text { mixed grain-size } \\
\text { sediments (clay } \\
\text { and/or silt and/or } \\
\text { sand, minor } \\
\text { gravel) }\end{array}$} & $\begin{array}{l}2 \text { to } 3 \\
3\end{array}$ & $\begin{array}{l}\text { clay, silt and } \\
\text { sand, minor } \\
\text { gravel }\end{array}$ & $T, U, S,(G)$ & $\begin{array}{l}\text { displaced loess and sandy } \\
\text { loess, sandy floodplain } \\
\text { deposits }\end{array}$ \\
\hline & 3 & $\begin{array}{l}\text { clay and/ or silt, } \\
\text { minor sand and } \\
\text { gravel }\end{array}$ & $\begin{array}{l}T, U,(S, G) \\
T-U,(S, G)\end{array}$ & $\begin{array}{l}\text { displaced loess, marginal fine- } \\
\text { grained limnic sediment, } \\
\text { crumbled clay }\end{array}$ \\
\hline \multirow{4}{*}{$\begin{array}{l}\text { D } \\
\text { mixed grain-size } \\
\text { sediments } \\
\text { (clay and/or silt } \\
\text { and/or sand } \\
\text { and/or gravel, } \\
\text { minor blocks) }\end{array}$} & \begin{tabular}{|l|}
2,3 \\
2 to 3 \\
\end{tabular} & $\begin{array}{l}\text { clay, silt, sand } \\
\text { and gravel }\end{array}$ & $\mathrm{T}, \mathrm{U}, \mathrm{S}, \mathrm{G}, \mathrm{X}$ & $\begin{array}{l}\text { solifluction material, } \\
\text { weathered hardrock }\end{array}$ \\
\hline & 2,3 & $\begin{array}{l}\text { clay, sand and } \\
\text { gravel }\end{array}$ & $\mathrm{T}, \mathrm{S}, \mathrm{G},(\mathrm{U})$ & solifluction material \\
\hline & $\begin{array}{l}2 \text { to } 3 \\
3\end{array}$ & $\begin{array}{l}\text { clay, silt and } \\
\text { gravel }\end{array}$ & $\mathrm{T}, \mathrm{U}, \mathrm{G}, \mathrm{S})$ & $\begin{array}{l}\text { solifluction material, clayey- } \\
\text { silty fan deposit, displaced } \\
\text { loess }\end{array}$ \\
\hline & \begin{tabular}{|l|}
2,3 \\
2 to 3 \\
\end{tabular} & clay and gravel & $\mathrm{T}, \mathrm{G},(\mathrm{U}, \mathrm{S})$ & solifluction material \\
\hline \multirow{3}{*}{$\begin{array}{l}\text { E } \\
\text { mixed grain-size } \\
\text { sediments (silt } \\
\text { and/or sand } \\
\text { and/or gravel } \\
\text { and/or blocks) }\end{array}$} & $\begin{array}{l}2 \text { to } 3 \\
2,3 \\
\end{array}$ & $\begin{array}{l}\text { clay and gravel, } \\
\text { minor blocks }\end{array}$ & $U, G,(X)$ & $\begin{array}{l}\text { silty fan deposit, silty fluvial } \\
\text { gravel }\end{array}$ \\
\hline & 2 & $\begin{array}{l}\text { silt, sand, gravel } \\
\text { and blocks }\end{array}$ & $U, S, G, X$ & clayey and blocky fan deposit \\
\hline & \begin{tabular}{|l|}
2,3 \\
2 to 3 \\
\end{tabular} & $\begin{array}{l}\text { silt, sand and } \\
\text { gravel }\end{array}$ & $U, S, G,(T)$ & $\begin{array}{l}\text { silty-sandy fan deposit, silty- } \\
\text { sandy fluvial gravel }\end{array}$ \\
\hline \multirow{4}{*}{$\begin{array}{l}\text { F } \\
\text { coarse-grained } \\
\text { sediments (sand } \\
\text { and/or gravel } \\
\text { and/or blocks) }\end{array}$} & 1,1 to 2 & sand and gravel & $\mathrm{S}, \mathrm{G}, \mathrm{X},(\mathrm{T}, \mathrm{U})$ & $\begin{array}{l}\text { sandy fluvial gravel, sandy fan } \\
\text { deposit }\end{array}$ \\
\hline & $1,(2)$ & pure sand & $\mathrm{S},(\mathrm{T}, \mathrm{U})$ & fluvial sand \\
\hline & 1 & gravel and blocks & $\mathrm{G}, \mathrm{X},(\mathrm{T}, \mathrm{U}, \mathrm{S})$ & coarse fluvial gravel \\
\hline & 1 & pure gravel & $\mathrm{G},(\mathrm{T}, \mathrm{U}, \mathrm{S})$ & fluvial gravel \\
\hline
\end{tabular}

Abbreviations: T: clay; U: silt; S: sand; G: gravel; X: blocks 
Table 1.2 Geotechnical unconsolidated sediments classification scheme for sedimants with organic components (Wagner, 2009).

\begin{tabular}{|c|c|c|c|}
\hline group & $\begin{array}{l}\text { geote } \\
\text { ch- } \\
\text { nical } \\
\text { classe } \\
\mathrm{s}\end{array}$ & Petrology & facies \\
\hline \multirow{10}{*}{$\begin{array}{l}\text { G } \\
\text { organogenetic sediments } \\
\text { or sediments with organic } \\
\text { constituents }\end{array}$} & \multirow[t]{3}{*}{4} & $\begin{array}{l}\text { slightly organic fine-grained } \\
\text { sediments ( } h 1, h 2 \text {, org, pf or fau) }\end{array}$ & $\begin{array}{l}\text { holocene soil, flood plain } \\
\text { deposits, limnic sediment, } \\
\text { displaced loess }\end{array}$ \\
\hline & & $\begin{array}{l}\text { slightly organic coarse-grained } \\
\text { sediments ( } \mathrm{h} 1, \mathrm{~h} 2 \text {, org, pf or fau) }\end{array}$ & $\begin{array}{l}\text { holocene soil, flood plain } \\
\text { deposits }\end{array}$ \\
\hline & & $\begin{array}{l}\text { slightly organic mixed grain-size } \\
\text { sediments ( } h 1, h 2 \text {, org, pf or fau) }\end{array}$ & $\begin{array}{l}\text { holocene soil, flood plain } \\
\text { deposits, limnic sediment, } \\
\text { displaced loess }\end{array}$ \\
\hline & \multirow[t]{3}{*}{4} & organic fine-grained sediments $(\mathrm{h})$ & $\begin{array}{l}\text { holocene soil, flood plain } \\
\text { deposits, limnic sediment, } \\
\text { displaced loess }\end{array}$ \\
\hline & & $\begin{array}{l}\text { organic coarse-grained sediments } \\
\text { (h) }\end{array}$ & $\begin{array}{l}\text { holocene soil, flood plain } \\
\text { deposits }\end{array}$ \\
\hline & & $\begin{array}{l}\text { organic mixed grain-size sediments } \\
\text { (h) }\end{array}$ & $\begin{array}{l}\text { holocene soil, flood plain } \\
\text { deposits, limnic sediment, } \\
\text { displaced loess }\end{array}$ \\
\hline & \multirow[t]{4}{*}{4} & $\begin{array}{l}\text { fine-grained sediments with high } \\
\text { organic content (h4) }\end{array}$ & $\begin{array}{l}\text { holocene soil, flood plain and } \\
\text { swamp deposits }\end{array}$ \\
\hline & & $\begin{array}{l}\text { coarse-grained sediments with high } \\
\text { organic content (h4) }\end{array}$ & $\begin{array}{l}\text { holocene soil, flood plain and } \\
\text { swamp deposits }\end{array}$ \\
\hline & & $\begin{array}{l}\text { mixed grain-size sediments with } \\
\text { high organic content (h4) }\end{array}$ & $\begin{array}{l}\text { holocene soil, flood plain and } \\
\text { swamp deposits }\end{array}$ \\
\hline & & organic clay & flood plain and swamp depoits \\
\hline $\begin{array}{l}\mathrm{H} \\
\text { freshwater limestone }\end{array}$ & 4 & freshwater limestone & sinter terraces \\
\hline
\end{tabular}

Abbreviations: h1: very slightly organic; h2: slightly organic; h or h3: organic; h4: strongly organic

The spatial structures and continuities are usually stronger in the startigraphic directions such as the layering surfaces.

The classes 5, 6, and 7 refer to the organic sediments, artificial fillings, and hard rock respectively which have not been explained in Table 1.1 and Table 1.2.

To take the general stratigraphic continuity directions into account and improve the spatial continuity structure model, a common technique is to model the facies and reservoir attributes in the modeling grids proportional between the top ad bottom correlation surfaces (Deutsch 2002, p. 88).

For that reason, the coordinate transformation was applied in this study. More details about the grids transformation will be discussed in the next sections. 


\subsection{An introduction to the geostatistical modeling methods (a comparison of different methods)}

\subsubsection{An overview to geostatistics}

\subsubsection{A bit of history:}

In the older conception, geostatistics had the meaning of the application of statistics in the geology or perhaps more generally in the earth sciences. In this sense, statistics was applied in the earth sciences since long time ago (Myers, 2002). Bertil Matern (born 1917) a forestry scientist and statistician introduced the meaning of the spatial variation and the importance of the spatial dependence application especially in the forestry science. In the mid 60th and especially in the mid 70th, the new meaning of the geostatistics was introduced with relation to the works of Georges Matheron (1930-2000). Matheron was influenced by Daniel J. Krige, a mining engineer in South Africa, and the former attempts of the Lev Gandin (1921-1927) a Russian Mathematician in the Leningrad Hydrogeological Institute. Krige was working on the assessments of the new gold mines using a limited number of boreholes. Matheron had theorized the important geostatistical concepts such as the regionalized variables. He was closely applying the developed methods in mining and petroleum applications. Matheron named the optimal estimation method of the unknown points using the available observations as "kriging" in the honor of Krige as a pioneer in geostatistics. Matheron not only explained the basics of Geostatistics for estimation especially for mining applications but he also paid attention to the quality of estimations and such concepts as estimation variance (Myers 2002; Myers 2008; Isaaks et al. 1989; and "Danie G. Krige." Wikipedia, Mar. 2012. Web. Apr. 2012).

\subsubsection{The estimation problem and geostatistics:}

A major problem in subsurface modeling is to estimate the attributes in the unknown points having a limited set of sample data, which can be employed for different cases such as geotechnical and geological applications. This estimation goal was primarily addressed by hand-contouring (in two-dimensional slices), providing an insight into the trends and uncertainty in data (Deutsch 2002, p. 154). 
Conventional estimation algorithms of the interpolation family, consider several simplifying assumptions such as constant values considered inside polygons, triangulation and linear interpolation inside the triangles (representing continuous changes), inverse distance weighted interpolation methods, etc.

These methods, for example the inverse-distance interpolation, are usually based on the estimation of the target variables in the unknown positions by means of a linear combination of neighboring known values. For such estimations, each of the known values of the surrounding points receives specific weights. For instance in the inversedistance method, the weights of the values inside a search radius for the known points applied for this estimation, are inversely proportional to their distance to the estimation point:

$$
\mathrm{Z} *(\mathrm{u})=\sum_{\mathrm{i}=1}^{\mathrm{n}} \frac{1}{\mathrm{~d}_{\mathrm{i}}} \mathrm{z}\left(\mathrm{u}_{\mathrm{i}}\right)
$$

Where $Z^{*}(u)$ is the estimating function for the location $u, z\left(u_{i}\right)$ is one of the neighboring samples used for estimation, and $d_{i}$ is the distance of the data from the estimation point.

To decide which method is the best among the others, some criteria can be defined and applied such as comparison of the univariate statistics of the estimates and true values, univariate distribution of errors, and evaluating the bivariate distribution of estimated and true values. Isaaks and Srivastava (1989) have compared polygonal estimation, triangulation, local mean, and inverse distance in some case studies (Isaaks et al., 1989) and represented some features about the efficiencies of these methods such as the existence of a global bias and smoothing effect (i.e. less variation in estimations in comparison to those of the true values)in all these methods. These two effects were less evident in polygonal estimates. The more samples were used in the estimation, the more smoothing were observed. On the other hand, since the triangulation method showed a more symmetric errors distribution, the lowest standard deviation, and the lowest interquartile range of the estimations, it was suggested as the best estimation method among the mentioned techniques to infer a sensible guess for the unknown points (Isaaks et al. 1989, pp. 249-277).

Some attempts were then conducted to perform optimal estimations in some objective sense. For instance, Daniel Krige was interested in, two criterions of the unbiasedness (correct overall average) as well as minimizing error variance in the estimations (Khojasteh 2002). For this purpose, kriging as an estimation method was developed by Georges Matheron (1961/62) which uses a linear combination of the known neighboring 
samples consistent with the two mentioned criteria. The kriging is usually abbreviated as the BLUE (the Best Linear Unbiased Estimator).

A regionalized variable is a variable (such as litofacies, porosity, or permeability) that may take different values at different spatial locations (Deutsch 2002, p. 345).

The two different terms of estimation and simulation have been often addressed in this context.

An estimation technique like kriging makes a single best guess of the unknown using the assumed spatial relationship between the data and the unknown and is locally accurate since it minimizes the local errors independent of what the global map of estimates may look like. The estimated maps usually represent much smoother outputs (Caers 2000, pp. 815-816).

The geostatistical simulation methods on the other hand, offer mapping methods which create non-unique and non-smooth results (Caers 2000, pp. 815-816). These methods are globally accurate rather than locally accurate. The conditional geostatistical simulation methods generate a set of possible equi-probable realizations which are conditioned to the available observations while having very close global statistical behaviors (e.g. variograms) to those of the reality. Having multiple simulated realizations, one is also able to evaluate the global uncertainties and risks.

\subsubsection{Some basic concepts in geostatistics}

There are some basic terms and concepts in geostatistics which should be introduced prior to any geostatistical discussion. Some of them such as; random function, random variable, discrete and continuous random variables, regionalized variable, and stationarity assumptions were described very briefly here:

\section{(a) Random function (RF):}

Geostatistics is basically founded on the concept of Random Functions (RF), by which the set of unknown attribute values $z(u)$ at each location of $u$, is regarded as a set of spatially dependent random variables (RV) (Goovaerts 1997, p. 63). With the concept of 
the random function, one can recognize and model structures in the spatial variation of the attribute (Goovaerts 1997, p. 63).

A group of spatially distributed samples can be considered as a realization of a random function (Matheron 1963).

\section{(b) Random variable (RV):}

According to Goovaerts (1997, p. 63), a random variable can be defined as following:

"A variable, that can take a series of outcome values according to some probability distribution".

A random variable can be either a discrete or a continuous random variable.

\section{(c) Regionalized variable $(\operatorname{ReV})$ :}

A variable distributed in space (or time) that can take different values in different spatial locations is termed as a regionalized variable (Deutsch 2002, p. 345). Geologic facies types or geotechnical soil types in different points are examples of categorical regionalized variables.

According to the regionalized variable theory (RVT), a set of regionalized variables includes three different relationships; structural part or trend, correlated variation, and uncorrelated variation or noise. In the geostatistics the main focus is on modeling the correlated variation element of the regionalized variable (Hattermann 2011). 


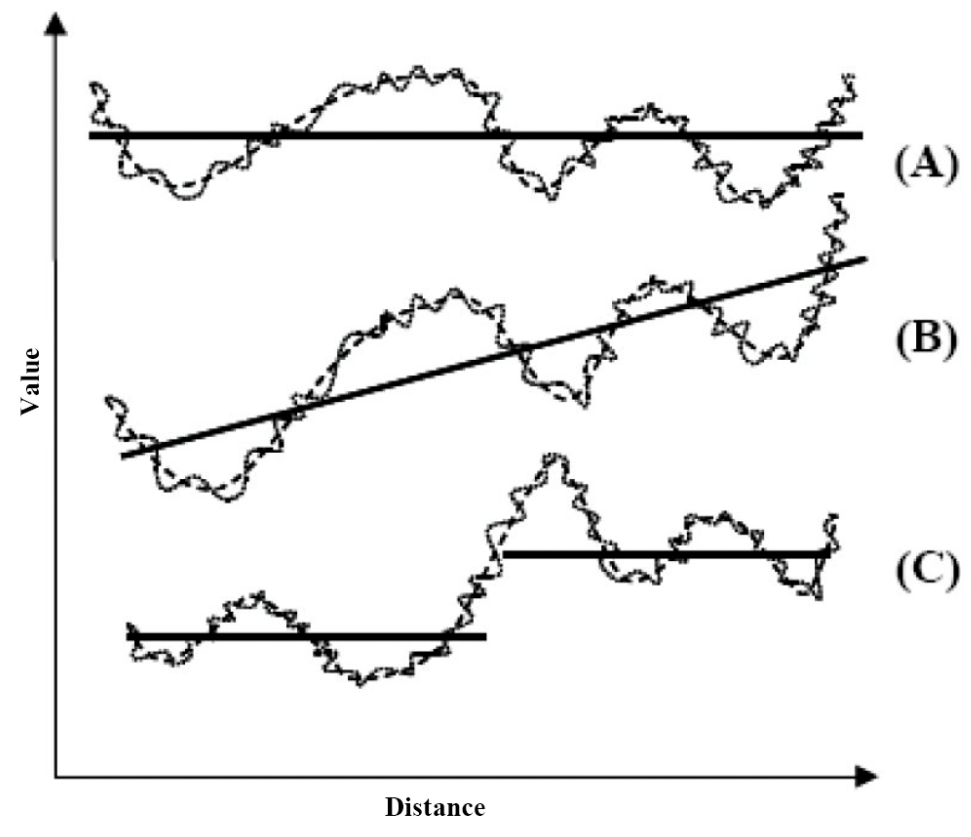

Figure 1.5 The stationarity of means for a regionalized variable, (A): referring to a stationary mean, (B): to a non-stationary mean with a trend, and (C): non-stationary case (Hattermann 2011, p. 17).

\section{(d) Stationarity assumptions:}

Stationarity is one of the most essential assumptions and decisions that should be considered for a random function in the geostatistical techniques. Stationarity implies that the statistics (such as mean, variance, covariance, and so on) is independent of the location of its calculation in the study area. For instance, a variogram can often be assumed stationary because it can be applied over the entire area which has been computed. The variogram in the circumstance of the stationarity, can be regarded a function of the separation vector (lag) between the pairs of points and independent of the location. It is the measure of spatial variability for separation vectors (Deutsch 2002, p. 346).

The stationarity is defined in terms of the first- and second-order moments of the samples random function, and stationarity degrees related to the special moments that stay unchanged in the study area (Hohn 1991, p. 23)

The expectation of the distribution function of a random variable $Z(u)$ at location $u$ is:

$$
E\{Z(u)\}=m(u)
$$

This expectation which is a first-order moment of the $Z(u)$ distribution can be dependent on $\mathrm{u}$. 
The four essential degrees of stationarity in geostatistics are; strict stationarity, secondorder stationarity, the intrinsic hypothesis, and quasi-stationarity. To define the stationarity assumptions easier, the concept of the spatial law of a random function can be introduced here. The spatial law of a random function can be termed as all observed distribution functions in all prospective locations of the study area. The referred stationarity assumptions have been explained briefly here as following:

1. Strict stationarity:

The spatial law between any pair of random variables $\left\{Z\left(u_{1}\right), Z\left(u_{2}\right) \ldots Z\left(u_{m}\right)\right\}$ and $\left\{Z\left(u_{1}+h\right), Z\left(u_{2}+h\right) \ldots Z\left(u_{m}+h\right)\right\}$ is invariant in the strict stationarity condition. In other words, the distribution function (of a random function) does not change in terms of the separation vector of $h$.

The strict stationarity assumption is not necessary in most of the geostatistical purposes since these applications often need only the first two moments (Hohn 1991, pp. 24-25).

\section{Second-order stationarity:}

The second-order stationarity requires:

(a) Invariant expectation:

$$
E\{Z(u)\}=m(u)=f i x
$$

(b) The covariance dependent only on the separation vector:

$$
C(h)=S \boldsymbol{Z}(u) \cdot Z(u+h) \rrbracket m^{2} \text { for each } u
$$

The stationarity of covariance $\mathrm{C}(\mathrm{h})$ is equal to the stationarity of the variance and semivariogram:

$$
\begin{aligned}
& \left.C(0)=E \mathbf{Z}(u)-m(u)^{2}\right]_{\frac{\gamma}{\mathbf{J}} \operatorname{Var} \boldsymbol{Z}(u)} . \\
& g(h)=E\{\mathbf{Z}(u+h)-Z(u)\} / 2=C(0)-C(h)
\end{aligned}
$$

Under the second-order stationarity conditions, the semivariogram or covariance can be chosen to measure the spatial auto- or cross-correlations (Hohn 1991, pp. 24-25).

3. The intrinsic hypothesis:

In the intrinsic hypothesis conditions, the expected first-moment and semivariogram values are invariant. Therefore, the intrinsic hypothesis is a reduced form of the second-order stationarity since it does not require the stationarity of the covariance 
but just the existence of a finite variance $\mathrm{C}(0)$. For most of the geostatistical applications, the intrinsic hypothesis is sufficient.

If the random variables and their functions do not conform to the conditions described above and therefore represent a kind of dependence of the functions on the location, they bear a kind of characteristics called 'trend' (Hohn 1991, pp. 24-25).

\section{Quasi-stationarity:}

The studied variables may in reality have a trend (or show a 'drift') over the study area. Although such a variable is not stationary in the insight of definitions mentioned above, a sort of local stationarity can be accepted in practice. This means that when the maximum applied distance in semivariogram or covariance calculation is much smaller relative to the trend range, the quasi-stationarity circumstance can be accepted (Hohn 1991, pp. 24-25).

\section{(e) Detecting the existence of a trend:}

Obviously, to identify the existence of a trend in regionalized variables, the most straightforward approach could be to plot the target variable against the distance in different directions and evaluate its general changes. To make a general trend clearer, the moving average or similar method can also be applied. In the case of the existence of a trend, a systematic change should be distinguished in the general variation of the values along the distance axes (similar to time-series analyses, e.g. Fahmy and Elsayed 2006; Smith 1989). As an example, suppose that there is a coal layer with variations in its thickness and the aim is to model and estimate the thickness of this layer. Generally, three types of variations in the thickness of the example layer could be identified; erratic, periodic, and systematic. Assume that, in general, the mentioned layer is getting thicker and thicker in a specific direction, let say from west to the east. However, in the local scale, the layer thickness shows some erratic changes while these changes have an algebraic sum of zero. The first part of the variations (i.e. the general systematic changes), are the trends of the investigated parameter. Therefore, plotting the thickness of the mentioned layer against the distance will represent how the thickness is increasing in the East-West direction in general, which can be modeled using a regression model. Subsequently, the thickness of the layer will be equal to the amount of the mentioned trend plus a local erratic variation: 


$$
Z(u)=m^{*}(u)+e
$$

where $\mathrm{Z}(\mathrm{u})$ stands for the amount of the required attribute (like thickness) in the location $\mathrm{u}, \mathrm{m}^{*}(\mathrm{u})$ is the estimated local average of that attribute, and $\mathrm{e}$ is the erratic term of the variation of the cited attribute or the error representing the difference between the estimation of general trend and the real values.

In the geostatistical methods like kriging, the local erratic variation term of the regionalized variable (i.e. $e$ equation (1-7)) can also be modeled.

All geological processes convey a sort of trend in distribution of petrophysical properties, such as fining or coarsening upward. Hence, the large-scale negative correlations indicative of a geologic trend is appeared as the variogram increase over the sill variance of $\mathrm{C}(0)=\sigma^{2}$ (Deutsch 2002, p. 117). From the above discussion, an important practical conclusion can be inferred that one of the best and most practical indicatives of the existence of a trend within the study zone is to plot the sample variogram and the theoretical sill, and evaluate the general behavior of the variogram plot relative to the theoretical sill. If the sample variogram increasingly exceeds the expected (theoretical) sill, the prevalence of a trend can be concluded. The expected sill can be considered as the variance of a data-set, i.e. $\mathrm{C}(0)=\sigma^{2}$, for the continuous variables, and $\mathrm{p}(1-\mathrm{p})$ for categorical variables where $\sigma^{2}$ is the continuous data variance and $\mathrm{p}$ is the proportion of a category in the categorical data. Another concept related to the trend is the areal trend that may cause a zonal anisotropy between the horizontal and vertical variograms. The concept of the zonal anisotropy has been discussed in the next sections. The areal trend can cause the vertical variograms to be unable to meet the full variability of the attributes. Therefore, the vertical variogram stay below the theoretical sill of the variogram. 


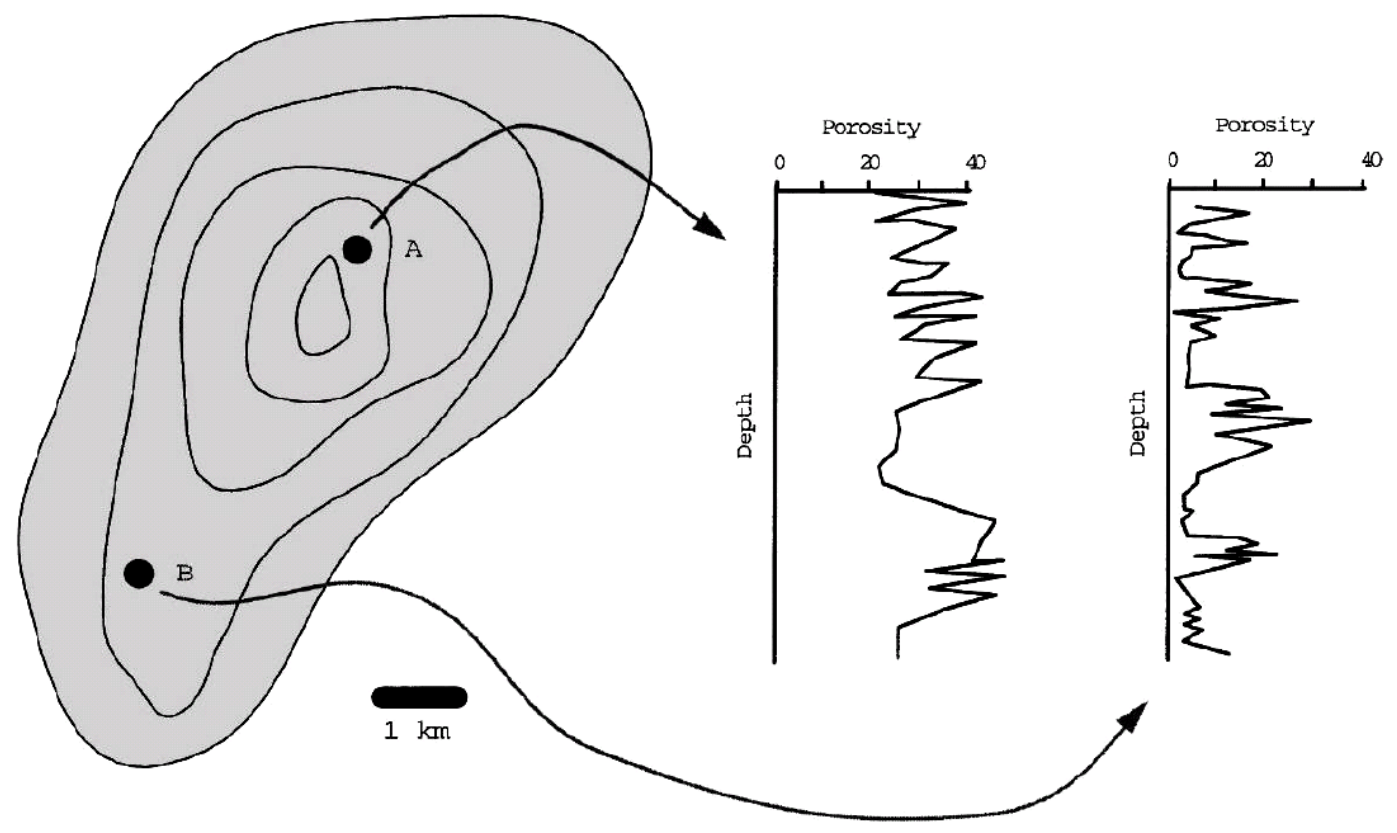

Figure 1.6 An example of the case of the presence of areal trends has been depicted here. In such cases, each well does not capture the full range of variability. In this example, well A faces mostly high values while the low values are observed in well B (from Gringarten and Deutsch 2001, p. 514).

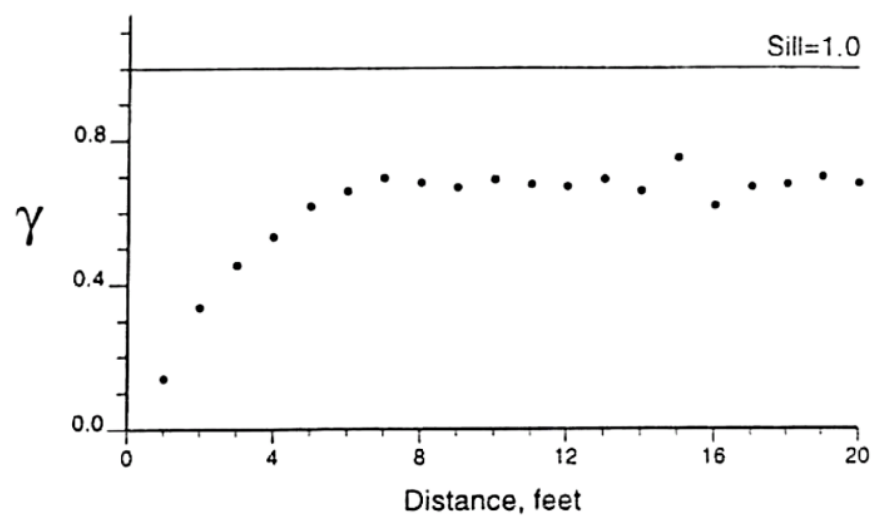

Figure 1.7 Vertical semivariogram with a zonal anisotropy in which the variogram does not reach its expected sill (Deutsch 2002, p. 121).

Figure 1.6 illiterates the effect of areal variability on the coverage of the variability of the attributes in the vertical direction (Deutsch 2002, pp. 121-122).

As discussed before, the first crucial stage in all geostatistical modeling procedures is to set the right model variables, to ensure, with an acceptable tolerance, that these characteristics can be modeled as a stationary variable along the study zone. In the case of the presence of a significant trend, the trend should be modeled and removed prior to any geostatistical modeling step. Finally, the trend can be added back to the estimations or 
simulation values over again. The action of removing the existing trends is called detrending. If a negligible trend is detected, the stationarity can be accepted and the regular geostatistical procedures can be followed (Deutsch 2002, p. 122).

The above discussions are more applicable to the continuous variables. Nevertheless, the same principles can be applied to the categorical variables modeling. As it will be explained in the next sections, the indicator geostatistical methods are usually applied in modeling the categorical variables. For such indicator geostatistical analyses, to check whether or not any trend exists, the proportions of the categories (e.g. facies or soil types) are considered instead of the variable means of the continuous variables. In other words, the proportions or existence probabilities of the categories are evaluated, their variations are modeled, and the unknown probabilities are estimated (or simulated). The trends in facies proportions usually exists and is likely in either areal or vertical directions and the trend models construction follows the same approach as for that of the continuous variables (Deutsch 2002, p. 186-187). Then the significant trends in proportions can be removed prior to the indicator geostatistical analyses. To have a rough evaluation of the probable existing trends in different parameters during this study, three approaches have been applied:

(a) Drawing the scatter-plots of each parameter against the distance.

(b) Plotting the location map of the boreholes which include the investigated parameters.

(c) Inspect the behavior of the indicator variograms for each soil category.

To achieve the best stationarity conditions, the zones and layers have been separated into the a few more similar and homogeneous sub-zones. For example, the deep holes were put aside and the slope sides of the basin were also considered separately.

\section{(f) Variograms and models:}

Modeling the regionalization is more beyond a simple smooth interpolation among the points. In order to do geostatistical modeling, it is necessary to explore the spatial variability in the existing data-set (Gringarten 2001). 


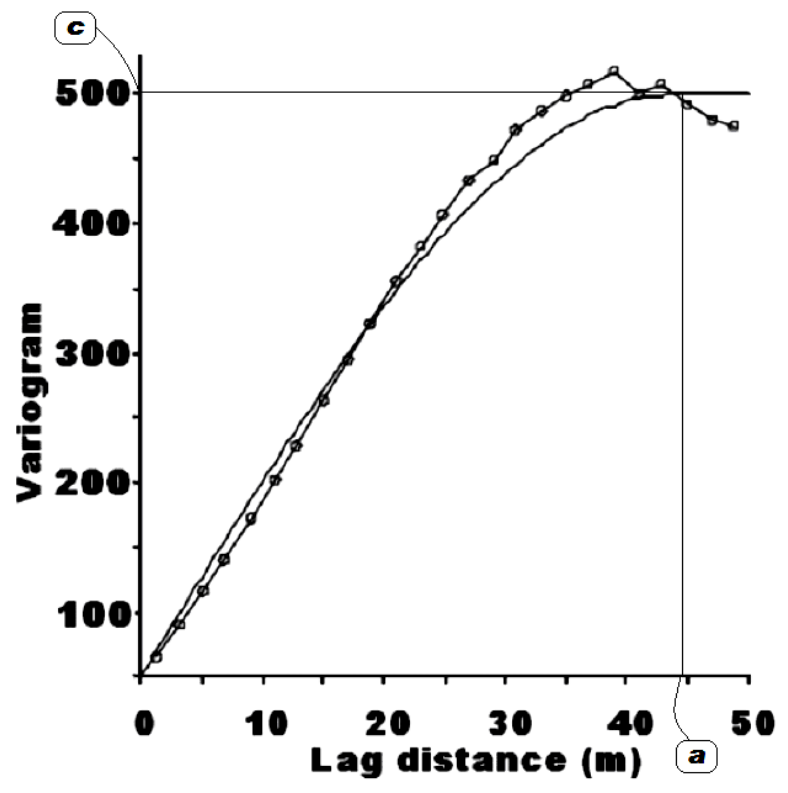

Figure 1.8 A typical variogram that can be applied in spatial visibilities modeling. The dots show the sample variogram and the solid curve represents the model variogram. The straight thin solid lines project the important variogram parameters of range (a) and sill (c).

Recalling the concept of the regionalized variable, the correlated stochastic component can be modeled in the next estimation or simulation stages. Obviously, any existing trends can be modeled and removed beforehand. Modeling the spatial variability structure of a regionalized variable can be conducted using various measures and methods such as twopoint statistics; including the covariograms or correlograms to measure the similarities, variograms or madograms as the measures of dissimilarities, and the multiple-point statistical methods that go beyond the two-point statistics in spatial variability modeling. These concepts have been elaborated in different sources and will not be addressed here except very briefly for variogram and its analytical models. A variogram which represents the degree of spatial dissimilarities for a regionalized variable can be expressed in mathematical terms as a mean variance of the pairs of the values separated with the separation vector of $h$, plotted for different separation vectors (Gringarten 2001). Similarly, this spatial correlation can be expressed among more than one variable using spatial cross-covariance functions (covariance of the variables with other variables) which are called covariograms. A typical variogram increases with the increase of the separation vector to a certain distance called range where the variogram will reach to a plateau called sill. The sill expectedly should be, in its full distance, nearly equal to the data 
variance for the continuous variable (i.e. $\gamma(0)=C(0)=\sigma^{2}$ ) and $p(1-p)$ for the indicator variograms. In this expression, p represents the proportion of the corresponding category.

A sample variogram of a regionalized variable therefore, can be defined as following:

$$
\left.\gamma^{*}(h)=\frac{1}{2 N(h)} \sum_{\alpha=1}^{N(h)} \mathbf{Z}\left(x_{\alpha}\right)-Z\left(x_{\alpha}+h\right)\right]^{2}
$$

where $\mathrm{N}(\mathrm{h})$ is the pairs number of the data locations which are separated by the vector $h$ (Isaaks 1989, p. 82). It should be considered that in most of the scientific sources, the term of "semivariogram" was applied for the above definition. However, for simplification reasons (as it has been used also in some other literature), it will usually be referred as "variogram" in this context.

Equally, if the indicator values (I), are replaced with regionalized variables of $\mathrm{Z}$ in the definition of the variogram, the sample indicator variogram will be expressed as following:

$$
\gamma_{I}^{*}(h)=\frac{1}{2 N(h)} \sum_{\alpha=1}^{N(h)}\left[\left(x_{\alpha}\right)-I\left(x_{\alpha}+h\right)\right]
$$

Such an indicator variogram is a valuable tool for modeling the spatial variability of indicator values in modeling categorical variables and estimating the local probability distribution functions. The act of fitting analytical models to the variograms is termed variography.

The above formulas provide a set of experimental values that reflect the spatial variability structure but neither in all distances nor in all directions. Hence, analytical models should be fitted to the experimental (or sample) variograms to; deduce the variogram values in all points, smooth out the effects of fluctuations, and ensure the positive definiteness property of variograms (Goovaerts 1997, p. 87; Deutsch 2002).

Positive-definiteness is an indispensable perquisite for solving the estimation equations of kriging to ensure that the kriging variance is positive and the estimation equations are solvable. Using specific standard mathematical variogram models to be fitted to the experimental variograms can ensure the positive-definiteness characteristic of the estimation models (Goovaerts 1997, p. 87; Deutsch 2002, p. 131). 
Pure nugget effect, spherical, exponential, Gaussian, and power models are the most frequently used basic variogram models. The mathematical definitions of the most important basic theoretical isotropic variogram models have been brought in different geostatistical resources. Yet, as an instance and one of the most important permissible mathematical variogram models, the equation for standardized isotropic spherical model has been brought here:

- Spherical model with a range of $a$ :

$$
g(h)=\operatorname{Sph}\left(\frac{h}{a}\right)=\left\{\begin{array}{l}
1.5 \cdot \frac{h}{a}-0.5 \cdot\left(\frac{h}{a}\right)^{3} \quad \text { if } \quad h<=a \\
1 \quad \text { otherwise }
\end{array}\right.
$$

To model the spatial variability of a regionalized variable, a composition of the mentioned models may offer a better fit. The first four models are bounded because they reach actually or practically to their sill (which was 1 in the above formula as their standardized sill). The real sill values can be considered in the models by multiplication of the sill values by the standardized models (Goovaerts 1997, p. 88).

The variograms may show different behaviors in different directions. This characteristic is recognized with the phenomenon of anisotropy (Sarma 2009; Goovaerts 1997). On the other hand, the structure of the data variations (e.g. variograms), does not change in different directions in an isotropic model. The variogram anisotropy can be of two major types; the geometric (also called elliptic), and zonal (also called stratified). In the geometric anisotropy, the variogram sills remain unchanged whereas their range changes in various directions. This sort of anisotropy can be illustrated with an anisotropy ellipse or ellipsoid and expressed by anisotropy ratio, for instance, in the case of twodimensional studies:

$$
b=\left(a_{1} / a_{2}\right)
$$

in which the $\mathrm{a}_{1}$ stands for the variogram range in one principal anisotropic direction and $\mathrm{a}_{2}$ is the variogram range in another principal anisotropic direction for a bounded variogram or:

$$
b=\left(\text { slope }_{1} / \text { slope }_{2}\right)
$$

for an unbounded variogram (Sarma 2009). 
With the anisotropy ratio, by a simple transformation, all the geostatistical equations can be reformulated based on the equations of the isotropic case.

Figure 1.9 illustrates the case of the geometric anisotropy in two variograms.
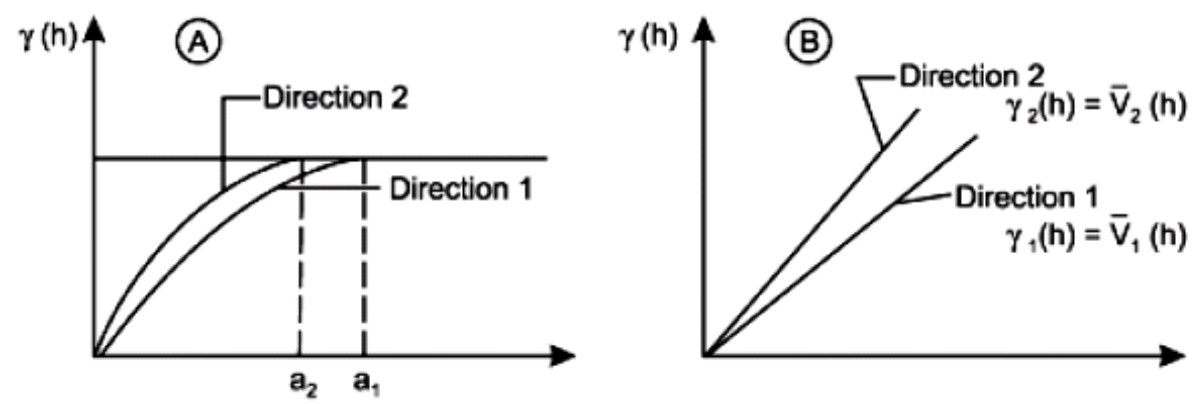

Figure 1.9 Anisotropic variograms; (A) variogram with the same sill and (B) linear variogram with different slopes (Sarma 2009, p. 83).
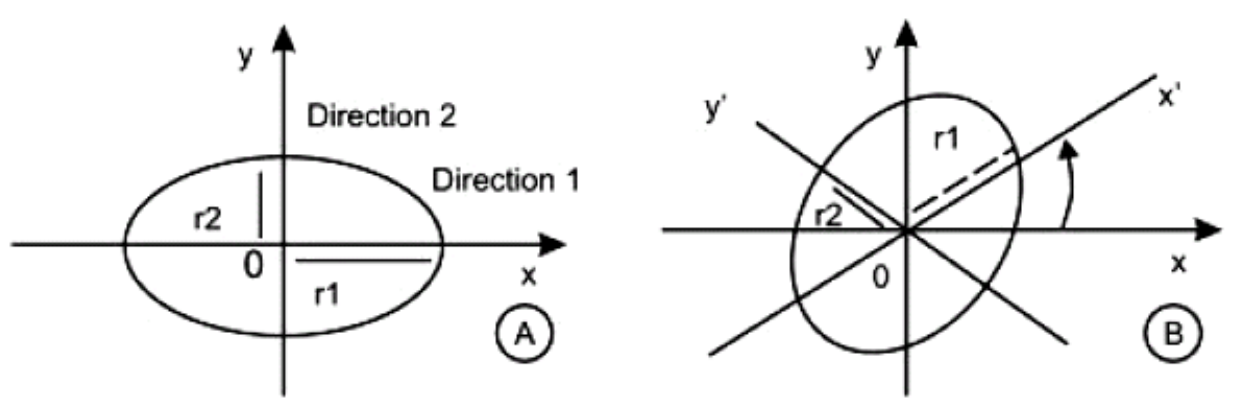

Figure 1.10 Samples of elliptical anisotropy; (A) main axes follow the direction of the co-ordinate axes and (B) main axes do not follow the direction of the co-ordinate axes (Sarma, 2009 p. 84).

In the case of zonal anisotropy, the sills are not equal in different directions, and in general, the variograms in various directions represent a complex behavior. Usually, in such cases, the variogram is split into two components; an isotropic variogram plus a variogram depending only on the vertical direction (Hohn 1999).

In this study, the zonal anisotropy between the horizontal and vertical variograms was obvious in indicator variograms. However, the problem was seen that the vertical indicator variograms could not meet all the variability because of the much shorter investigation distance. The component of the vertical indicator variograms played the role of the nugget effect for the horizontal indicator variograms. Therefore, the threedimensional variograms were the sum of the two mentioned components. 


\subsubsection{Kriging and geostatistical simulation basics}

Let's start with an example to address the importance and advantage of using the kriging estimator in practical problems, especially in the case of subsurface studies where the access and information are more limited.

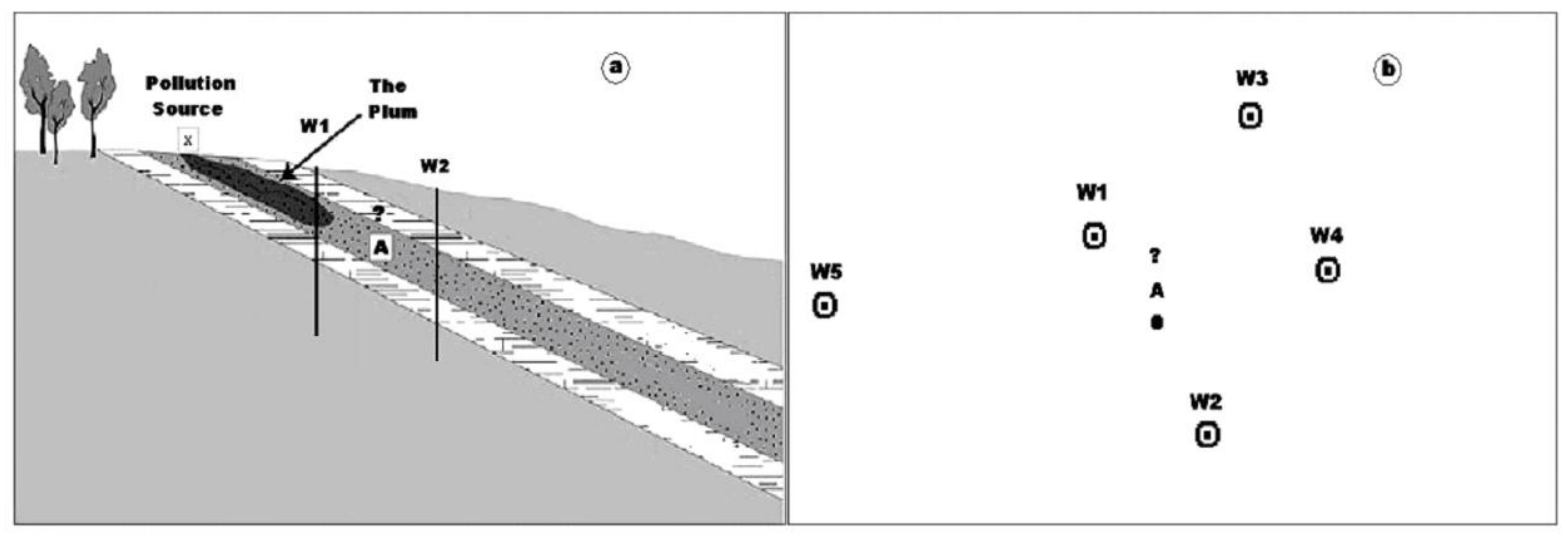

Figure 1.11 An illustration of the subsurface estimation problem in environmental applications. $W_{1}$ to $W_{5}$ represent the drilling locations for getting subsurface samples or measurements.

Suppose that in an environmental survey, the monitoring of the extent and immigration of contaminants and proper remediation planning is aimed. Yet, due to the technical and economical limitations, it is almost impossible to have a full measurement of the required parameters and characteristics throughout the subsurface. In this example, assume that, all the available and reliable information and data could be achieved through a limited number of samples taken from some boreholes $\left(\mathrm{W}_{1}\right.$ to $\left.\mathrm{W}_{5}\right)$.

Therefore, the required characteristics are known in a few points but they are unknown elsewhere which is the majority of the investigation zone and the prediction of the facies types within which the mentioned characteristics are targeted would be the first step in modeling.

As it has already been discussed, there are different approaches to estimate the required characteristics in the unknown points which fall in two main categories of structure imitating and process-imitating. In the structure-imitating methods, the observed patterns and structures are modeled without directly considering the processes responsible of their creations. The process-imitating techniques are not the issue of this study (Falivene et al. 2007, p. 200; Koltermann and Gorelick 1996).

The kriging aim is to achieve the "Best Linear Unbiased Estimation" (B.L.U.E.) by assigning weights to surrounding samples to an estimation point (Clark 1979, p. 100). In 
comparison to the conventional estimation techniques, kriging takes also the spatial variability structure, the samples distribution patterns, and the probable anisotropies into account.

Although kriging is basically developed with the linear underlying presumptions, the non-linear estimation frameworks can also be defined in the kriging approach. These nonlinear estimations are termed non-linear kriging which fit better to the most practical applications since most data sets do not obey the multivariate Gaussian assumptions (Hohn 1999, pp. 134-135).

Kriging estimator contains two essential properties (Armstrong 1998, p. 92; Deutsch 2002, p. 154):

(a) Unbiasedness, which means that the average of the estimation errors (the difference of real and estimated values) are zero :

$$
\mathrm{E}\left[\mathrm{z}_{\mathrm{v}}-\mathrm{z}_{\mathrm{v}}^{*}\right]=0
$$

in which $z^{*}$ is estimated and $z$ is the true value.

(b) Minimum estimation error, which means that the average squared difference between the true and estimated values (square error) should be minimum:

$$
\sigma_{\mathrm{E}}^{2}=\mathrm{E}\left[\left(\mathrm{z}_{\mathrm{v}}-\mathrm{z}_{\mathrm{v}}^{*}\right)^{2}\right] \text { minimum }
$$

These equations obey stationarity assumptions.

Based on the type of the mean of a regionalized variable in kriging equations, kriging is categorized into different types such as; simple kriging (SK) in which the mean is constant and known, ordinary kriging $(\mathrm{OK})$ in which the mean is constant but unknown, and universal kriging (UK) where the mean is unknown and inconstant.

When a stationarity or quasi-stationarity conditions is met, using the OK (ordinary kriging) or SK (simple kriging) would be suitable. However, in the case of significant trends, detrending should be performed first. In this case, using the ordinary or simple kriging was identified to be adequate due to the stationarity or quasi-stationarity conditions in the existing data-set of the soil types.

The final equations of the kriging with the ordinary kriging conditions (i.e. constant and unknown mean of the variable) only are brought here. 
Rewriting the kriging equations for ordinary kriging, the estimation equation can be summarized as:

$$
\mathrm{z}_{\mathrm{v}}^{*}=\sum_{\mathrm{i}=1}^{\mathrm{n}} \lambda_{\mathrm{i}} \mathrm{Z}_{\mathrm{v}_{\mathrm{i}}}
$$

where $z_{v}^{*}$ is the estimate value based on the linear combination of $Z_{v_{i}}$ known neighboring values and $\lambda_{i}$ are kriging weights and $n$ is the number of neighboring points. The result of equation (1-15) will be:

$$
\sum_{i=1}^{n} \lambda_{i}=1
$$

and a set of equations for ordinary kriging can be written as:

$$
\left\{\begin{array}{l}
\sum_{j=1}^{n} \lambda_{j} \bar{C}\left(v_{i}, v_{j}\right)-\mu=\bar{C}\left(v_{i}, v\right) \quad i=1,2, \ldots, n \\
\sum_{j=1}^{n} \lambda_{j}=1
\end{array}\right.
$$

$\overline{\mathrm{C}}\left(\mathrm{V}_{\mathrm{i}}, \mathrm{V}_{\mathrm{j}}\right)$ in the above equations, is the average covariance between pairs of points and can be calculated using auxiliary functions. Therefore, having covariance or variogram functions (i.e. variogram models), a set of $n+1$ equation exist with $n+1$ unknown parameters including $\mathrm{n}, \lambda_{i}$ kriging weights and a Lagrange parameter.

In the indicator geostatistical approaches, a value so-called indicator is assigned to each sample point so that the indicator is set to one (1) when the category exists at that point and zero (0) otherwise, i.e. :

$$
I_{k}(u)=\left\{\begin{array}{lll}
1 & \text { if } \quad c l(u)=k \\
0 & \text { if } \quad c l(u) \neq k
\end{array} \mathrm{k}=1,2, \ldots \mathrm{n}\right.
$$

where $c l(u)$ is the true class that exists in the location $u, k$ is a category of soils between 1 and $n$, and $n$ is the number of existing categories.

The same variography and estimation methods are used for the indicators to achieve the estimations and simulation. The outcome of the indicator kriging, therefore, is the probabilities of the occurrence of each class at each location. In this study, the assigned category to each estimation point from the indicator kriging was the soil class that held the highest probability of occurrence estimated from indicator kriging. 
Although, the indicator kriging method is suitable in estimation of the probability of the occurrence of the facies, it faces some critical problems in modeling and estimating such as; the estimated values out of $(0,1)$ range (nonsense probabilities) and vague experimental variograms (because of insufficient data ) that are difficult and uncertain to model.

As referred before, kriging includes a problem so called smoothing effect. As a result, the global statistics of the estimations (including the univariate statistics, e.g. mean and histogram, and bivariate statistics e.g. variogram and covariogram) are different from those of the real statistics. In some practical problems such as flow simulating inside a hydrocarbon reservoir or detecting points with high vulnerability to contamination, the smoothing effect becomes a major drawback for kriging methods since the subtle variations are very important in such cases (Yamamoto, 2005).

Geostatistical simulation is an alternative to overcome the smoothing effect problem by producing a set of equi-probable results which resemble the reality in terms of the global behaviors and reproducing the fine variations. This set of realizations can be evaluated later as different possible scenarios for the reality (Caers 2005, p. 13).

Geostatistical simulation methods can be categorized into two broad groups of sequential and non-sequential as well as two main groups of conditional and unconditional methods. In the sequential simulation methods, the simulation is started from a simulation grid node and goes forward. Each time, the input data and the previously simulated values are considered for the simulation of the next grid node. The sequential indicator simulation method is an example of sequential simulation methods in geostatistics. In the nonsequential methods on the other hand, the simulation is performed all at once, such as the LU decomposition and p-filed simulation methods (Caers 2005).

In conditional simulation, the local conditional cumulative density function (CCDF) is approximated in each simulation node of the simulation grid and a random variable is drawn out of it, then using the existing data and already simulated points, the simulation is followed in a random path which visits all the simulation nodes. More details about the conditional geostatistical simulation are brought in relevant literature.

Sequential Gaussian (SGSIM) and Sequential indicator simulation (SISIM) methods are among conditional geostatistical simulation methods which are broadly used in various applications. However, SGS and SIS have some shortcomings. In SGSIM, the Gaussian assumption is not always reliable or readily verifiable. On the other hand, SISIM faces the 
same difficulties as indicator kriging in modeling and statistics faces; i.e. tedious and from time to time vague variogram models, order-relation violations and sometimes nonrealistic probabilities inferred from indicator kriging. For example, the estimated probability may become greater than one or less than zero. Another problem of the SISIM method is the difficulty of considering the inter-class relations among the categories. Considering the inter-class correlation can certainly improve the estimation quality especially for in the case of limited number of samples. Although one also may employ the methods like cokriging that take the inter-class correlations into account. However, cokriging method requires some constraints and special conditions in building the covariogram models that make its application more complicated.

Some geostatistical simulation methods based on Markov chains, which have been applied in the coming sections, have paved the way to improve the modeling of variability and to overcome the problems included in the traditional geostatistical methods.

In general, according to the author's opinion and knowledge, some remarkable problems in the traditional geostatistical methods should be taken into consideration, such as variogram modeling in the lack of sufficient data, insufficiency of two-point statistics in capturing complexities, severely mathematical view in traditional methods, linearity of estimators, and etc.

In the coming section, some geostatistical methods have been compared together based on the literature review.

\subsubsection{A comparison of some geostatistical modeling (estimation/simulation) methods, considering their applications:}

During the literature review according to the advisors' recommendations, plenty of different papers and books were reviewed aiming at finding the strength and weaknesses of different geostatistical modeling methods and selecting their best in terms of the applicability of the method in such cases, availability of the software, and the efficiency of the method.

Among a big variety of reviewed geostatistical modeling and simulation techniques, only the most important of them have been pointed out here. 
Table 1.3 summarizes the basics, applications, advantages, disadvantages, and software of some geostatistical methods which were reviewed in the initial stage of this research to compare and choose the most suitable of them for applying in this study.

The reviewed methods can be put into the following groups:

(1) Markov-chain based methods: for example: transition probability-based Markov chain (TP/MC), coupled Markov chain (CMC), triple Markov chains (TPC), Markov chain geostatistics (MCG), hierarchical architecture modeling of continuous characteristics using Markov chains (Ritzi 2007).

(2) Multiple-point statistics (MPS).

(3) Bayesian maximum entropy (BME).

Because, bringing the complete summary of all the corresponding discussions here would be too tedious and lengthy to and to make it easier and quicker to compare and access all of the reviewed methods together with their most important features, the required information about them have been summarized in Table 1.3.

\subsubsection{Summary and highlights of the compared methods}

The most important points about the mentioned comparison of the various geostatistical modeling methods can be summarized as following:

(a) Modeling the boundaries of categories prior to modeling their containing continuous variables would lead to significant improvements in the resulting models.

(b) Most of the mentioned Markov chains-based models facilitate the integration of subjective and interpretive information (such as geology and expert's interpretations) into the models and result in considerable improvement in modeling spatial continuity.

(c) Stochastic simulations based on Markov chains can lessen some shortcomings of conventional geostatistical methods theoretically because of:

$\circ$ Being non-linear.

o Being neither completely stochastic nor completely deterministic (intermediate stochastic). 
o Facilitating consideration of the inter-class correlations.

o Reducing the order-relation problem of $\mathrm{IK}^{1}$.

(d) Two-point statistics such as variograms can not completely capture the spatial variability complications, especially when heterogeneity has a rather complex geometry. MPS ${ }^{1}$ is a solution to overcome this problem.

(e) Considering a general primary knowledge and updating the posterior distribution function like in $\mathrm{BME}^{1}$ method seem very effective ways of improving stochastic modeling.

(f) Traditional geostatistical approaches such as IK, suffer from several problems such as order-relation violations and the weakness in complex subsurface heterogeneities reconstruction specially about modeling spatial connectivity among them and particularly when samples are sparse (Bierkens and Weerts 1994, p. 283; Li and Zhang 2008, p. 158).This might be due to difficulty in making inter-class correlations and being linear (Li and Zhang 2008, p. 158).

(g) Although the proposed methods based on the direct use of Markov chains (MCs) have significant advantages over other methods especially over traditional geostatistical methods, they still may have some shortcomings such as these:

o Because they are among unilateral processes, they may generate inclined patterns (i.e. diagonal trends) due to the asymmetric neighborhoods used in multidimensional simulations. To reduce or avoid this problem, symmetric or quasi-symmetric path may be defined (Davis 2002, p. 575). However, the transition-probability Markov chain (TP/MC) approach applied in the present study does not implement asymmetric paths and expectedly should not produce such problems

o Pattern inclinations and artifacts are possible to occur. This is especially about $\mathrm{CMC}^{2}$ method when asymmetric or quasi-asymmetric paths are not used. They may also occur in MCG. In addition, using an asymmetric or quasiasymmetric path may reduce the positive feature of considering asymmetry as an advantage of these methods (Park et al. 2007, p. 910).

$\circ$ In $\mathrm{TPC}^{2}$ and $\mathrm{MCG}^{2}$ methods, the main formulation is suited for twodimensional problems. The direct and easy formulations of these methods for

\footnotetext{
${ }^{1}$ IK stands for indicator kriging, MPS multiple-point statistics, and BME for Bayesian maximum entropy.

${ }^{2} \mathrm{CMC}$ stands for coupled Markov chain, TPC for triple Markov chains, MCG for Markov chain geostatistics, and TPs for transition-probabilities.
} 
three-dimensional problems and with considering three-dimensional modeling scheme are hardly mentioned except for CMC that suffers other problems.

o In CMC, underestimation of small classes and overestimation of big classes especially when samples are few and sparse may cause unrealistic patterns.

o Some other problems in CMC, TPC and MCG include; difficulties with accounting for anisotropies and large-scale features (e.g. in MCG because of the use of small, systematic neighborhood system), practical problems with calculating TPs particularly from sparse data, the problem of inferring multistep TPs form one-step TPs and some theoretical simplification problems such as conditional independence assumptions ( $\mathrm{Li}$ and Chuanrong 2008, pp. 159$163)$.

(h) BME and MPS seem to be too dependent on external information sources rather than available data-set, such as training images, interpretations, and so on.

(i) Most of the mentioned methods have been applied in specific practical problems while they can be applied in the other fields as well. It seems that the main difference is due to abundance and the sort of available data in different applications, e.g. in mining problems, various and abundant data usually exists whereas there is little data in environmental problems. However, in special cases, the problems may become more similar. For instance, in the primary exploration stages of hydrocarbon reservoirs, little information may exist.

Some other geostatistical methods such as truncated Gaussian and truncated plurigaussian methods as well as cleaning cell-based methods for modeling the soil lithofacies can also be applied for modeling the geotechnical soil classes. The first two methods are based on generating the realizations of one or more Gaussian continuous random variable and truncating them in specific thresholds to achieve the required categorical variables. More details and their comparison with the techniques applied in this study have been brought in Chapter 7 .

In general, due to the practical and theoretical situation of this project, the best proposed method among the mentioned methods according to the literature should be TP/MC method. 
Table 1.3 Practical summary and comparison of some geostatistical methods based on main framework and the reasons of using them.

\begin{tabular}{|c|c|c|c|c|c|}
\hline Method group & $\begin{array}{l}\text { The reason for using this } \\
\text { method group }\end{array}$ & $\begin{array}{l}\text { Method } \\
\text { subgroup }\end{array}$ & $\begin{array}{l}\text { Why this method, where, and } \\
\text { the philosophy of its } \\
\text { application? }\end{array}$ & $\begin{array}{c}\text { Main basics and assumptions } \\
\text { of the method }\end{array}$ & $\begin{array}{l}\text { Main framework of the } \\
\text { method }\end{array}$ \\
\hline \multirow{4}{*}{$\begin{array}{l}\text { Markov Chains } \\
\text { (MC) }\end{array}$} & \multirow{4}{*}{$\begin{array}{l}\text { 1. Intermediate between } \\
\text { completely stochastic and } \\
\text { deterministic processes, i.e. the } \\
\text { processes is expected to be only } \\
\text { partially stochastic. }{ }^{1} \\
\text { 2. Non-linear. } \\
\text { 3. Probable dependency } \\
\text { between variables in time and } \\
\text { space and the Walter's law of } \\
\text { facies succession about } \\
\text { geological or similar sequences. } \\
\text { 4. Easier integration of } \\
\text { geological data into models. }{ }^{2} \\
\text { 5. Easier cross-correlations. }{ }^{3}\end{array}$} & $\begin{array}{c}\text { Transition probability-based } \\
\text { indicator geostatistics (TP/MC) }\end{array}$ & $\begin{array}{l}\text { 1. Improving spatial continuity } \\
\text { modeling, taking some geological } \\
\text { information into account. } \\
\text { 2. To overcome vague indicator } \\
\text { variography. } \\
\text { 3. Avoid some IK problems like order } \\
\text { relation violations. } \\
\text { 4. Incorporating geological/physical } \\
\text { information in modeling. } \\
\text { 5. Suitable for categorical data. } \\
\text { 6. Asymmetry can be considered. }\end{array}$ & $\begin{array}{l}\text { 1. Existence of Markov property. } \\
\text { 2. All IK equation can be rewritten in } \\
\text { terms of transition probabilities. }{ }^{.} \text {. } \\
\text { 3. Using conceptual information like } \\
\text { mean length or proportion of a facies } \\
\text { can help us to make better spatial } \\
\text { continuity models. }\end{array}$ & $\begin{array}{l}\text { 1. Get, estimate or calculate the mean } \\
\text { length and proportion of each different } \\
\text { lithofacies. } \\
\text { 2. Calculate the 3D transition } \\
\text { probabilities in different distances and } \\
\text { between each } 2 \text { categories. } \\
\text { 3. The model parameters of transition } \\
\text { probabilities or (co)variograms can be } \\
\text { modeled using the proportions and the } \\
\text { mean lengths of each category. } \\
\text { 5. Perform IK using TP equations. } \\
\text { 6. Perform SISIM using TP equations. } \\
\quad \text { Simulated annealing also can be } \\
\text { used to improve the simulations. }\end{array}$ \\
\hline & & Coupled Markov chain (CMC) & $\begin{array}{l}\text { 1. Straight-forward modeling and } \\
\text { easier conditioning to available data. } \\
\text { 2. Taking geological information into } \\
\text { account. }\end{array}$ & $\begin{array}{l}\text { 1. Existence of Markov property. } \\
\text { 2. Conditional independence of } \\
\text { samples.7 } \\
\text { 3. Two single MCs (in } 2 \text { D or } 3 \text { single } \\
\text { chains are forced to move to equal } \\
\text { states. }^{7}\end{array}$ & $\begin{array}{l}\text { 1. Calculating the Markovian vertical } \\
\text { TPs using borehole data and inferring } \\
\text { the horizontal TPs using Walther's law } \\
\text { (transiograms and modeling them). } \\
\text { 2. Making grids and Inserting well data } \\
\text { in their location as conditioning data. } \\
\text { 3. Calculating the probability of } \\
\text { occurring each states, using the relevant } \\
\text { equation; (e.g. for } 2 D \text { problem with } \\
\text { Equation. (1-26)) row-wise and from } \\
\text { top to bottom and determining the } \\
\text { succeeding state (which would be } \\
\text { drawn from the CDF of all states at that } \\
\text { point, randomly). } \\
\text { 4. Filling out the remaining cells one by } \\
\text { one with similar procedure. }\end{array}$ \\
\hline & & $\begin{array}{l}\text { Triplex Markov chain } \\
\text { geostatistics (TMC) }\end{array}$ & $\begin{array}{l}\text { 1. In soil problems with no sparse } \\
\text { samples and soil line measurements. } \\
\text { 2. Avoiding trends in produced patterns } \\
\text { despite CMC method. }\end{array}$ & $\begin{array}{l}\text { 1. The same assumptions of CMC } \\
\text { 2. The simulation path is modified to } \\
\text { avoid directional effects and trended } \\
\text { patterns. } \\
\text { 3. Divide area to windows inside } \\
\text { survey lines and walk in two opposite } \\
\text { directions. } \\
\text { 4. Uses } 2 \text { CMSs from } 3 \text { independent } 1 \text { - } \\
\text { D MCs. }\end{array}$ & $\begin{array}{l}\text { 1. Is according to the same basics of } \\
\text { CMC. } \\
\text { 2. Two CMCs from three independent } \\
\text { CMCs are used, i.e. from right to } \\
\text { left, from left to right and from top } \\
\text { to bottom. } \\
\text { 3. Applying the simulation inside } \\
\text { windows that are partitioned inside } \\
\text { survey lines, row-wise in opposite } \\
\text { directions. }\end{array}$ \\
\hline & & $\begin{array}{l}\text { Markov chain geostatistics } \\
\text { (MCG) }\end{array}$ & $\begin{array}{l}\text { 1. Direct application of MC in } \\
\text { simulation (esp. soil problems) } \\
\text { 2. To overcome small-class } \\
\text { underestimation problem of CMC. }\end{array}$ & $\begin{array}{l}\text { 1. Only one single MC exists in space } \\
\text { which has its CPD in each location } \\
\text { entirely dependent on its nearest } \\
\text { known neighbours in different } \\
\text { directions.9 } \\
\text { 2. The conditional independence is } \\
\text { theoretically correct in Pickard (1980) }\end{array}$ & $\begin{array}{l}\text { 1. Applies a similar framework as the } \\
\text { CMC but it uses only the nearest } \\
\text { known points of estimation points } \\
\text { and a single MC rather than coupling } \\
\text { MCs. }{ }^{10} \\
\text { 2. Transition probabilities among each } \\
\text { pairs of classes are calculated, based }\end{array}$ \\
\hline
\end{tabular}




\begin{tabular}{|c|c|c|c|c|c|}
\hline & & & & $\begin{array}{l}\text { random field for sparse samples, when } \\
\text { the nearest known neighbours in } \\
\text { cardinal directions are considered. }{ }^{9}\end{array}$ & $\begin{array}{l}\text { on the distances and modeled. } \\
\text { 3. The CPD (conditional probability } \\
\text { distribution) is calculated using the } \\
\text { equation }(* *) \text {. } \\
\text { 4. Drawing a random class, based on } \\
\text { the above CPD and continuing the } \\
\text { procedure. }\end{array}$ \\
\hline & & $\begin{array}{l}\text { Hierarchical architecture } \\
\text { modelling (by: R. Ritzi) }\end{array}$ & $\begin{array}{l}\text { 1. Incorporating analogue data } \\
\text { (outcrops...) } \\
\text { 2. Improving semivariogram } \\
\text { modelling of continuous variables (e.g. } \\
\text { permeability) and its estimation by } \\
\text { univariate statistics, transition } \\
\text { probabilities and proportions. } \\
\text { 3. When Sediments show hierarchical } \\
\text { structure. } \\
\text { 4. As usually more categorical data than } \\
\text { continuous is available. }\end{array}$ & $\begin{array}{l}\text { 1. Sediments usually represent } \\
\text { hierarchical structure. } \\
\text { 2. The permeability variogram is a } \\
\text { linear summation of the auto } \\
\text { variograms and cross variograms for } \\
\text { defined unit types, weighted by } \\
\text { proportions and transition probabilities } \\
\text { of unit types. }{ }^{11} \\
\text { 3. The sample variogram can be } \\
\text { approximated using only cross- } \\
\text { transition probabilities and the } \\
\text { univariate statistics (mean and variance) } \\
\text { for permeability within unit types. }{ }^{12} \\
\end{array}$ & $\begin{array}{l}\text { 1. Determine and classify the different } \\
\text { levels of hierarchies of sedimentary } \\
\text { facies. } \\
\text { 2. Calculate the auto- and cross- } \\
\text { transitions and proportions of each } \\
\text { category. } \\
\text { 3. Calculating the univariate statistics } \\
\text { (variance and mean) of continuous } \\
\text { parameter (e.g. permeability) in } \\
\text { different hierarchical levels. } \\
\text { 4. Having the calculated parameters, } \\
\text { one can approximate the variogram } \\
\text { model for continuous variable. }\end{array}$ \\
\hline \multirow{2}{*}{$\begin{array}{l}\text { Multiple-point statistics } \\
\text { (MPS) }\end{array}$} & \multirow{2}{*}{$\begin{array}{l}\text { Two-point statistics such as } \\
\text { variograms can not capture the } \\
\text { complex heterogeneities }\end{array}$} & MPS ( continuous) & \multirow{2}{*}{$\begin{array}{l}\text { 1. Bivariate statistic (e.g. variograms) } \\
\text { can not capture complex } \\
\text { heterogeneities (e.g. meanders, } \\
\text { channel (sand) bodies...). } \\
\text { 2. Solve the problems of object-based } \\
\text { methods e.g. too much time-consuming, } \\
\text { limitations when sampling is dense } \\
\text { relative to average object size. }\end{array}$} & \multirow{2}{*}{$\begin{array}{l}\text { 1. Analogue data can help us to make } \\
\text { complicated heterogeneity models. } \\
\text { 2. Proper training images can help us to } \\
\text { fill information gaps. } \\
\text { Stationarity and ergodicity assumptions } \\
\text { should be considered (for training } \\
\text { images, etc.). }\end{array}$} & \multirow{2}{*}{$\begin{array}{l}\text { 1. Finding the most general prior } \\
\text { distribution (no specific). } \\
\text { 2. Considering specific knowledge, } \\
\text { including hard and soft data. } \\
\text { 3. Information from stages } 1 \text { and } 2 \text { is } \\
\text { processed by means of logical data. }{ }^{14}\end{array}$} \\
\hline & & MPS (categorical) & & & \\
\hline \multirow{2}{*}{$\begin{array}{c}\text { Bayesian maximum entropy } \\
\text { (BME) }\end{array}$} & \multirow{2}{*}{$\begin{array}{l}\text { Easier integration of different } \\
\text { sources of information and } \\
\text { physical or expert's knowledge } \\
\text { or interpretation. } \\
\text { Suitable for little data } \\
\text { condition. }\end{array}$} & BME (continuous) & \multirow{2}{*}{$\begin{array}{l}\text { 1. Offers a nonlinear model. } \\
\text { 2. Taking different sources of } \\
\text { information (hard and soft) into } \\
\text { account. }^{15}\end{array}$} & \multirow{2}{*}{$\begin{array}{l}\text { 1. Available hard \& soft data and } \\
\text { expert's knowledge can be applied. } \\
\text { 2. Maximizing the entropy maximizes } \\
\text { the information. } \\
\text { 3. Posterior probabilities can be } \\
\text { analyzed to get favourite } \\
\text { assessments. }\end{array}$} & \multirow{2}{*}{$\begin{array}{l}\text { 3. Finding the most general prior } \\
\text { distribution (no specific). } \\
\text { 4. Considering specific knowledge, } \\
\text { including hard and soft data. } \\
\text { 3. Information from stages } 1 \text { and } 2 \text { is } \\
\text { processed by means of logical data. }\end{array}$} \\
\hline & & BME (categorical) & & & \\
\hline
\end{tabular}




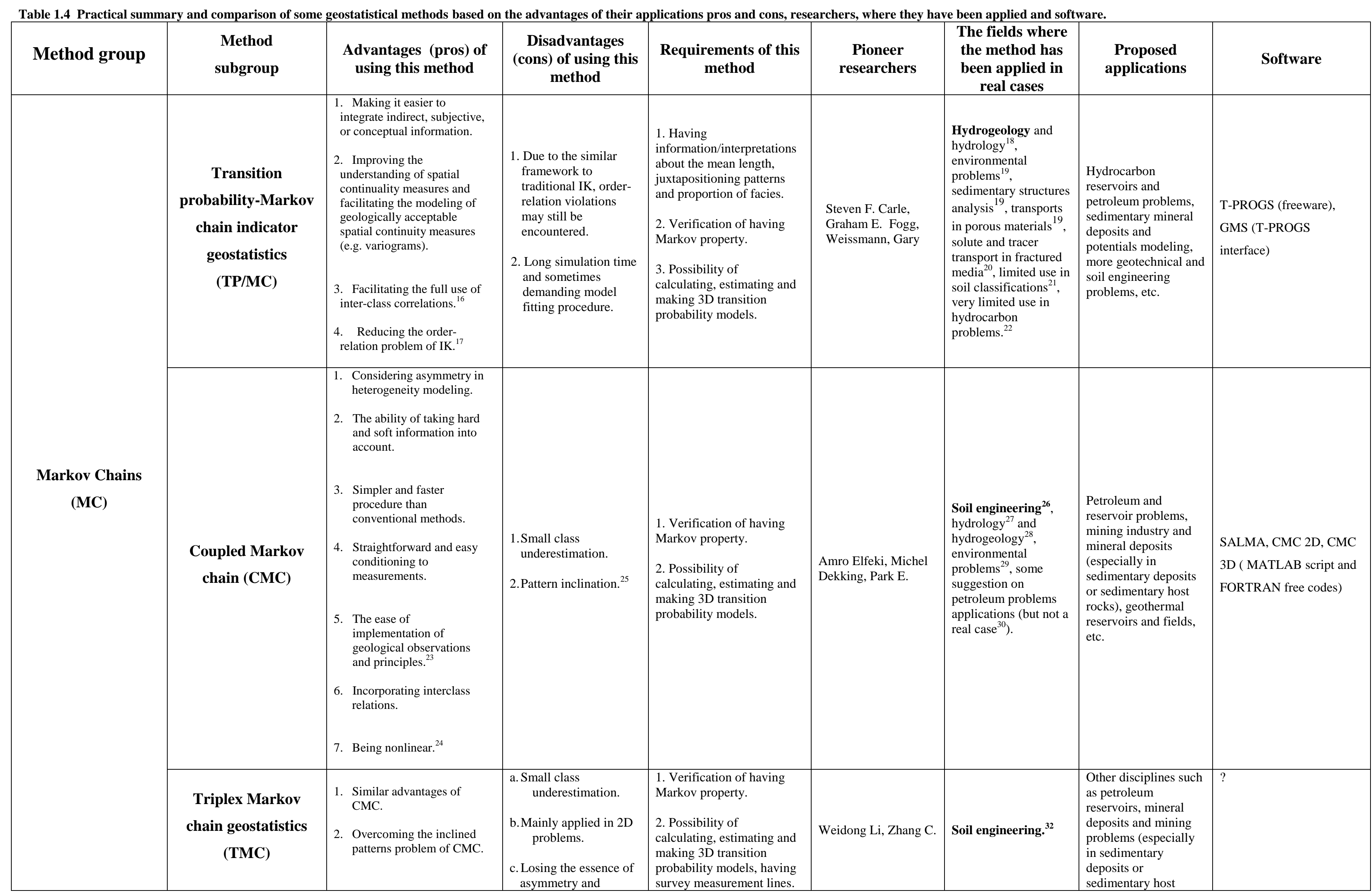




\begin{tabular}{|c|c|c|c|c|c|c|c|c|}
\hline & & & $\begin{array}{l}\text { directional } \\
\text { dependency of MC. }{ }^{31}\end{array}$ & & & & $\begin{array}{l}\text { rocks) and } \\
\text { geothermic... }\end{array}$ & \\
\hline & $\begin{array}{c}\text { Markov chain } \\
\text { geostatistics (MCG) }\end{array}$ & $\begin{array}{l}\text { 1. Similar advantages of } \\
\text { CMC. } \\
\text { 2. To a much extent, } \\
\text { overcoming the inclined } \\
\text { patterns problem of CMC. } \\
\text { 3. Overcoming the small- } \\
\text { class underestimation } \\
\text { problem of CMC. }\end{array}$ & $\begin{array}{l}\text { 1. For categorical } \\
\text { variables. } \\
\text { Difficulties in taking } \\
\text { a secondary variable } \\
\text { into account. }{ }^{33} \\
\text { 3. So far, developed for } \\
\text { two-dimensional } \\
\text { applications. }{ }^{34} \\
\text { 4. Probable inclined } \\
\text { patterns. }\end{array}$ & $\begin{array}{l}\text { 1. Verification of having } \\
\text { Markov property. } \\
\text { 2. Possibility of calculating, } \\
\text { estimating and making 3D } \\
\text { transition probability } \\
\text { models. }\end{array}$ & Weidong Li, Zhang C. & $\begin{array}{l}\text { Soil engineering }{ }^{35} \text {, } \\
\text { and limited } \\
\text { applications in water } \\
\text { problems. }{ }^{36}\end{array}$ & $\begin{array}{l}\text { Other disciplines such } \\
\text { as petroleum } \\
\text { reservoirs, mineral } \\
\text { deposits and mining } \\
\text { problems (especially } \\
\text { in sedimentary } \\
\text { deposits or } \\
\text { sedimentary host } \\
\text { rocks) and } \\
\text { geothermic... }\end{array}$ & $?$ \\
\hline & $\begin{array}{c}\text { Hierarchical } \\
\text { architecture } \\
\text { modeling (by: R. } \\
\text { Ritzi) }\end{array}$ & $\begin{array}{l}\text { 1. Suitable for continuous } \\
\text { parameters. } \\
\text { 2. Taking analog information } \\
\text { and hierarchical structure } \\
\text { into account. } \\
\text { 3. Suitable when more } \\
\text { categorical datat than } \\
\text { continuous is available. } \\
\text { 4. Improving variogram } \\
\text { modeling. } \\
\text { 5. No need to curve- } \\
\text { fitting procedure. }\end{array}$ & $\begin{array}{l}\text { Due to following the } \\
\text { conventional framework } \\
\text { of first making } \\
\text { variograms the } \\
\text { performing kriging..., } \\
\text { faces similar problems } \\
\text { of traditional methods. }\end{array}$ & $\begin{array}{l}\text { 2. Having hierarchical } \\
\text { structures. } \\
\text { 2. Verification of having } \\
\text { Markov property. } \\
\text { 3. Possibility of } \\
\text { calculating, stimating and } \\
\text { making 3D transition } \\
\text { probability models. } \\
\text { 4. Having } \\
\text { information/interpretation } \\
\text { about univariate statics } \\
\text { such as mean length and } \\
\text { proportion of categories } \\
\text { and variance of } \\
\text { continuous variable. }\end{array}$ & $\begin{array}{l}\text { Ritzi, R. W.; Dai, ZX; } \\
\text { Dominic, DF }\end{array}$ & $\begin{array}{l}\text { Hydrogeology } y^{38}, \\
\text { solute transport and } \\
\text { environmental } \\
\text { problems. }{ }^{39}\end{array}$ & $\begin{array}{l}\text { Other disciplines such } \\
\text { as petroleum } \\
\text { reservoirs, mineral } \\
\text { deposits and mining } \\
\text { problems (especially } \\
\text { in sedimentary } \\
\text { deposits or } \\
\text { sedimentary host } \\
\text { rocks) and } \\
\text { geothermics, etc. }\end{array}$ & $\begin{array}{l}\text { Free FORTRAN codes and } \\
\text { parameter files are } \\
\text { available by Robert Ritzi's } \\
\text { group. The file have } \\
\text { GSLIB format. }\end{array}$ \\
\hline $\begin{array}{l}\text { Multiple-point } \\
\text { statistics } \\
\text { (MPS) }\end{array}$ & MPS ( continuous) & $\begin{array}{l}\text { 1. Ability of complex } \\
\text { heterogeneity } \\
\text { reproduction. } \\
\text { 2. Ability of incorporating } \\
\text { different sources of } \\
\text { information such as } \\
\text { analogs and interpretations } \\
\text { into account. }\end{array}$ & $\begin{array}{l}\text { 1.Necessity of having } \\
\text { training images. } \\
\text { 2.Computationally } \\
\text { costly. } \\
\text { The high effect of } \\
\text { training image rather } \\
\text { than available } \\
\text { information. }\end{array}$ & $\begin{array}{l}\text { Having a suitable training } \\
\text { image from analogs or } \\
\text { interpretations. } \\
\text { Considering the } \\
\text { stationarity, ergodicity and } \\
\text { scale and direction } \\
\text { parameters properly. }\end{array}$ & $\begin{array}{l}\text { Strebelle, S.; Caers, } \\
\text { J.; Journel, A. }\end{array}$ & $\begin{array}{l}\text { Hydrocarbon } \\
\text { reservoirs and } \\
\text { petroleum } \\
\text { problems } \\
\text { hydrogeoological } \\
\text { problems. }\end{array}$ & $\begin{array}{l}\text { Other disciplines such } \\
\text { as hydrogeology, } \\
\text { mineral deposits and } \\
\text { mining problems and } \\
\text { geothermics, etc. }\end{array}$ & $\begin{array}{l}\text { snesim is an algorithm } \\
\text { available in S-GEMS v. } 3 \\
\text { (freeware under MS } \\
\text { Windows system), } \\
\text { developed in C++ }\end{array}$ \\
\hline $\begin{array}{c}\text { Bayesian maximum } \\
\text { entropy (BME) }\end{array}$ & BME (categorical) & $\begin{array}{l}\text { 1. Avoiding unwanted values } \\
\text { in estimation such as } \\
\text { negative estimation where } \\
\text { they are impossible. } \\
\text { 2. Nonlinear estimator. } \\
\text { 3. Ease of taking primary } \\
\text { knowledge and different } \\
\text { sources of information into } \\
\text { account. } \\
\text { 4. No presumptions about } \\
\text { data distribution nature. } \\
\text { 5. Producing a set of } \\
\text { realizations and post- } \\
\text { processing. }\end{array}$ & $\begin{array}{l}\text { Misleading estimates are } \\
\text { possible when soft data } \\
\text { is of interval form. }{ }^{42}\end{array}$ & $\begin{array}{l}\text { 1. Having some idea } \\
\text { about primary knowledge } \\
\text { of parameters. } \\
\text { 2. Gathering different } \\
\text { sources of information } \\
\text { including hard and soft } \\
\text { data e.g. in interval forms } \\
\text { or distribution functions... }\end{array}$ & $\begin{array}{l}\text { Christakos, George, } \\
\text { Bogart Patrik, Serre } \\
\text { Mac, D’Or Dimitri }\end{array}$ & $\begin{array}{l}\text { Physical and medical } \\
\text { geography, human } \\
\text { exposure, earth and } \\
\text { atmospheric sciences, } \\
\text { environmental } \\
\text { engineering, } \\
\text { epidemiology, health } \\
\text { sciences, risk } \\
\text { assessment, and } \\
\text { decision analysis. }{ }^{43} \text {. }\end{array}$ & $\begin{array}{l}\text { Other disciplines such } \\
\text { as petroleum } \\
\text { reservoirs, mineral } \\
\text { deposits and mining } \\
\text { problems ... }\end{array}$ & $\begin{array}{l}\text { BMELib and its } \\
\text { graphical user interface: } \\
\text { SEKS-GUI } \\
\text { BMELib is a free code } \\
\text { developed in MATLAB }\end{array}$ \\
\hline
\end{tabular}


The abbreviations of the table:

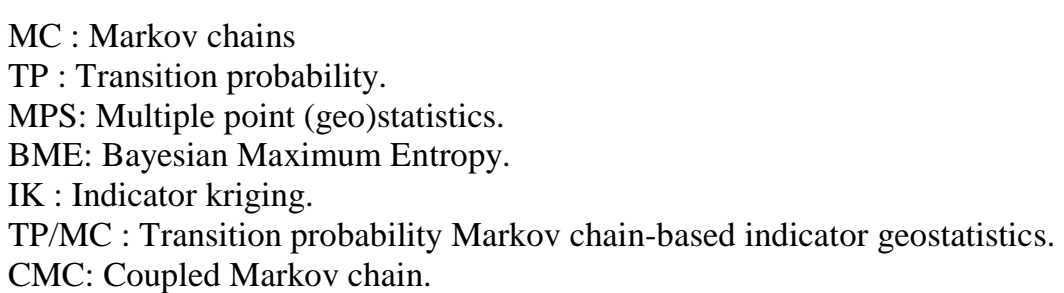

TMC: Triplex Markov chain geostatistics.

\begin{tabular}{|l|l|l|l|l|l|l|l|l|l|}
\hline 1,1 & & & & & & & & & $\mathrm{Nx}, \mathrm{l}$ \\
\hline & & & & & $\mathrm{i}, \mathrm{j}-1$ & & & & \\
\hline & & & & $\mathrm{i}-1, \mathrm{j}$ & $\mathrm{i}, \mathrm{j}$ & & & & $\mathrm{Nx}, \mathrm{j}$ \\
\hline $\mathrm{l}, \mathrm{Ny}$ & & & & & & & & & $\mathrm{Nx}, \mathrm{Ny}$ \\
\hline
\end{tabular}

Figure 1.12 The definition of the simulation grid and cell indexes for coupled Markov chain methods in equation (1-26) (Figure adapted from Elfeki and Dekking 2001, p. 574).

Equation (1-26): $p_{l m, k \mid q}=\operatorname{Pr}\left(Z_{i, j}=S_{k} \mid Z_{i-1, j}=S_{l}, Z_{i, j-1}=S_{m}, Z_{N_{x, j}}=S_{q}\right)=\frac{p_{l k}^{h} \cdot p_{k q}^{h\left(N_{x}-i\right)} \cdot p_{m k}^{v}}{\sum_{f} p_{l f}^{h} \cdot p_{f q}^{h\left(N_{x}-i\right)} \cdot p_{m f}^{v}}$

In this equation, $p_{l m, k \mid q}$ stands for the probability of transition from class $l$ in point $(\mathrm{i}-1, \mathrm{j})$ in row, and class $m$ in point (i, j-1) in column, to the class $k$ in point (i, j) given that the class (i.e. state) in the rightmost side (on the borehole) is $q, Z_{a, b}$ shows the state (i.e. class) in point (a, b), $S_{c}$ represents the state $c$ of a point, $p_{d e}^{h}$ shows the probability of transition from state $d$ to state $e$ in horizontal direction, and $p_{o p}^{v}$ for the probability of transition from state $o$ to state $p$ in vertical direction.

${ }^{6}$ Carle 1999, p. 46.

Elfeki and Dekking 2001, p. 573

Park et al. 2005, pp. 194-196; Elfeki and Dekking 2001, pp. 578-579.

Li et al. 2007, p. 325 
${ }^{10}$ Li 2007, pp. 326-329.

${ }^{11}$ Dai et al. 2005, p. 1.

${ }^{12}$ Dai et al. 2005, p. 5; Ritzi et al. 2004, pp. 9-10.

${ }^{13}$ Strebelle 2003, p. 1.

${ }^{14}$ Christakos 2000, p. 90; D'Or 2003, p. 20.

${ }^{15}$ Christakos 2000, p. 250.

${ }^{16}$ Carle 2008, pp. 3-4.

${ }^{17}$ Carle and Fogg 1996, p. 472.

${ }^{18}$ Lee et al. 2007 and Fleckenstein et al. are examples.

${ }^{19}$ Rubin et al. 1996 as an example.

${ }^{20}$ Park et al. 2004 as an example.

${ }^{21}$ D'Or 2004, and Bennett 2006 as examples.

${ }^{22}$ For example in Bohling and Dubois 2005.

${ }^{23}$ Park et al. 2005, p. 1.

${ }^{24}$ Li 2007, pp. 573-577.

${ }^{25} \mathrm{Li} 2007$, p. 576.

${ }^{26}$ Park et al. 2007 as an example.

${ }^{27}$ Elfeki 2006-a as an example.

${ }^{28}$ Dekking et al. 2001 as an example.

${ }^{29}$ Elfeki 2006-b as an example.

${ }^{30}$ Elfeki et al., 2002 as an example.

${ }^{31}$ Park et al. 2007, p. 910.

${ }^{32} \mathrm{Li}$ et al. 2004 as an example.

${ }^{33} \mathrm{Li}$ and Zhang 2007, p. 578.

${ }^{34} \mathrm{Li}$ and Zhang 2011; Li and Zhang 2007 as examples.

${ }^{35}$ Öli and Zhang 2008 as an example.

${ }^{36} \mathrm{Li}$ et al. 2001 as en example.

${ }^{37}$ Dai et al. 2005, p. 11.

${ }^{38}$ Sun et al. 2008 as an example.

${ }^{39}$ Ramanathan et al. 2008 as an example.

${ }^{40}$ Caers et al. 2000 and Hoffman et al. 2005 as examples.

${ }^{41}$ Feyen and Caers 2006, Huysmans and Dassargues 2009 as examples.

${ }^{42}$ Orton and Lark 2007, p. 68.

${ }^{43}$ Christakos 2000. 


\section{The general workflow of the geostatistical subsurface modeling in Göttingen test site}

Considering the mentioned advantages of using geostatistical methods, the traditional geostatistical analyses as well as the transition-probability Markov chain (TP/MC) method were examined in this study.

The main objective of the study was to evaluate the capability of using geostatistical methods in modeling the subsurface categorical parameters.

It should be emphasized here again that the investigated sediments below the surface and above the hard rock was referred as "soil" in this context.

The first step was to classify the soil samples on the basis of the DIN 18196 German standard for geotechnical soil classifications. For each soil class, a code was given between 1 and 5 (though, only classes 1 to 4 exists in limits of present study zone) representing its geotechnical class according to Table 1.1 and Table 1.2.

Then, the study zones were chosen and separated. The separation of the zones was done to achieve a better stationarity as well as to take some geological information into consideration.

Along each borehole, the data of the observed soil types was then re-sampled in every $0.5 \mathrm{~m}$ and stored in a file. Then, to each re-sampled point, the indicator values $\left(\mathrm{I}_{\mathrm{k}}\right)$ were assigned for every soil class. The estimation and sample coordinates were moreover transformed proportional to the top and bottom surfaces of the investigated layer. Afterward, the experimental indicator variograms of each soil class in different directions were calculated and modeled. As expected, an important zonal anisotropy between the horizontal and vertical directions of the variograms of each soil category was obvious. Subsequent to fitting models to sample variograms in various directions, threedimensional variogram models of each soil class were inferred considering the mentioned anisotropy. The probability of the occurrence of each soil class then was calculated using the indicator kriging (IK) method. In each estimation point, the class that held the highest estimated probability (from the IK method) was assigned as the estimated soil class to that location. Finally, the estimation grid (i.e. the relative stratigraphic coordinate) was back- transformed to the original one. The estimation models were also validated an improved when necessary. These (variogram) models were applied to generate geostatistical simulations using the sequential indicator simulation method (SISIM). The realizations of the SISIM method were then assessed for their quality of statistics 
reproductions and geological soundness. The best produced realizations were also chosen based on the statistical and geological criterions. Similarly, the transition probability Markov chain method was performed by calculating and modeling the transitionprobabilities among all soil classes.

The Markov chain models were fitted, after that, to the sample transition-probabilities of the soil categories. Then some steps of simulation optimizations (simulated annealing or quenching) were also performed. Figure 2.1 summarizes the mentioned stages for the present analyses.

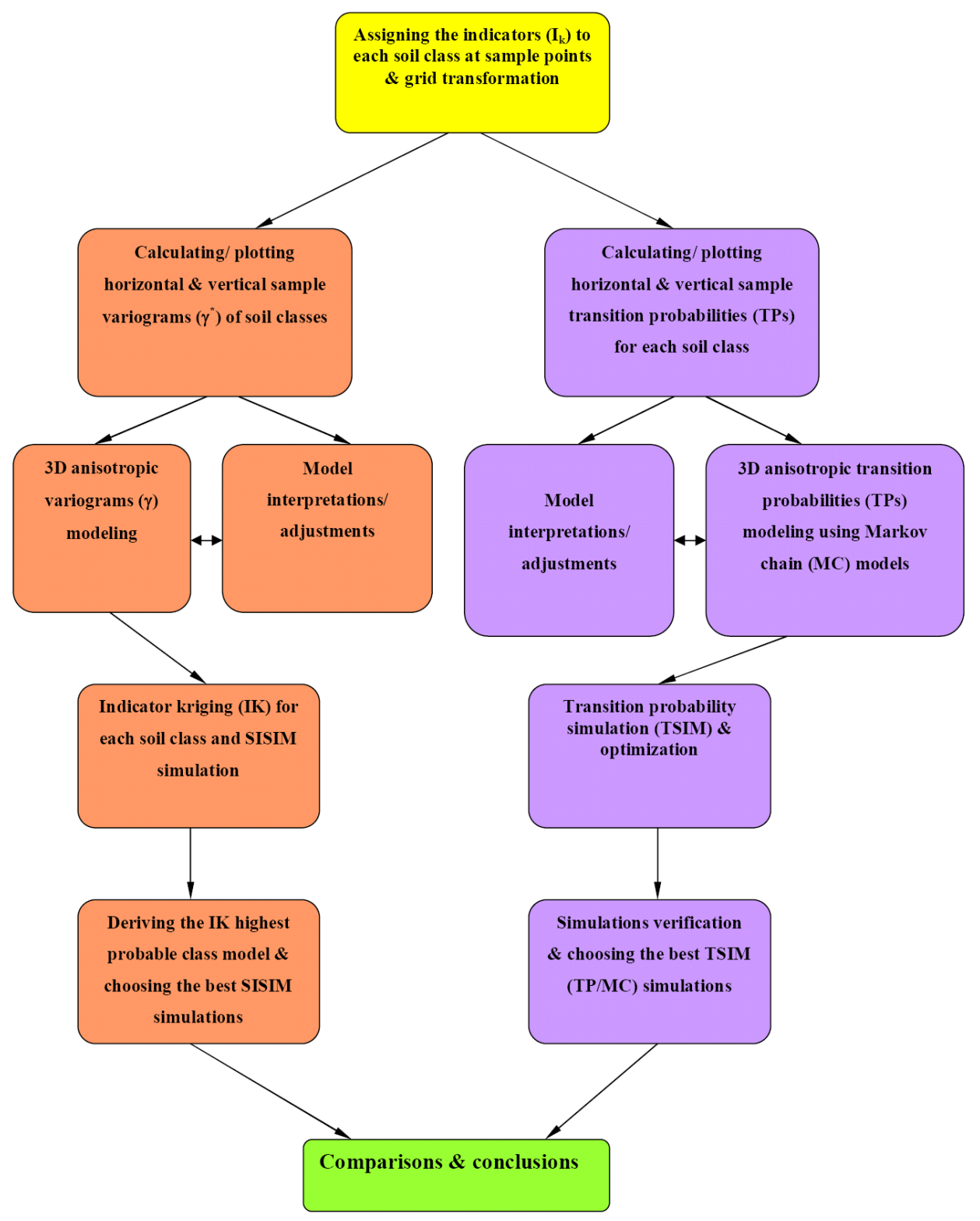

Figure 2.1 General workflow of geostatistical modeling stages of Göttingen test site. 


\subsection{The investigation site and data}

As explained before, the study area is located near the city of Göttingen in Lower Saxony province (Niedersachsen) of Germany (see Figure 1.1).

A part of the whole area was selected as a test site to evaluate the capabilities of the geostatistical modeling methods in similar modeling projects. The study site was then separated into some sub-zones to achieve better modeling results.

Modeling of all parts of the test site, at once, might ignore some points about geostatistical modeling perquisites such as:

(a) Geological reliability: assigning the characteristics of geologically too different zones in one geostatistical modeling round, might lead to inaccurate models in geological point of view.

(b) Stationarity: the first basic assumption in all geostatistical analysis is stationarity. Therefore, the modeling zones must be as homogenous as possible and the average frequency of each soil class must be as constant as possible over the modeling zone.

For those reasons, it is geostatistically more appropriate to differentiate stationary subvolumes prior to the modeling practice. Since the separation of the reasonable geoengineering sub-volumes had already been accomplished by a comprehensive interpretative manual preprocessing of the available borehole data, as well as the derivation of a structural model, the application of the pre-defined layers was not an unusual practice. In addition, these layers were defined according to their geologicalgenetical relations, which ensure a fine linkage between the geological and geotechnical characteristics.

Initially, a pilot area with the X-coordinate ranging from 355800 to 3567000 meters and Y- coordinate ranging from 5710925 to 5712500 meters is selected to examine the efficiency of the proposed methods. In addition, to certify the geostatistical stationary conditions in the models, the study zone was limited to the Pleistocene layer 5.

Figure 2.2 represents the locations of the boreholes in three-dimensional view. Figure 2.4 and 2.5 also demonstrate the location map of the boreholes and the mode class of the soil categories (cluster numbers) in each borehole inside the mentioned layer. In other words, in this two-dimensional map, the classes of the soils with the highest frequency in every 
borehole, is illustrated. Therefore, it can reflect how the major prevailing soil classes are distributed across the study zone in a two-dimensional view.

Then it was decided to limit the study part to the eastern basin of the mentioned site and the slope sides of the basin were cut and the study zone was limited to the $\mathrm{X}$-coordinate ranged from 3560022 to 3566746 and the $\mathrm{Y}$-coordinate ranged from 5710925 to 5712500 meters (Figure 2.5).

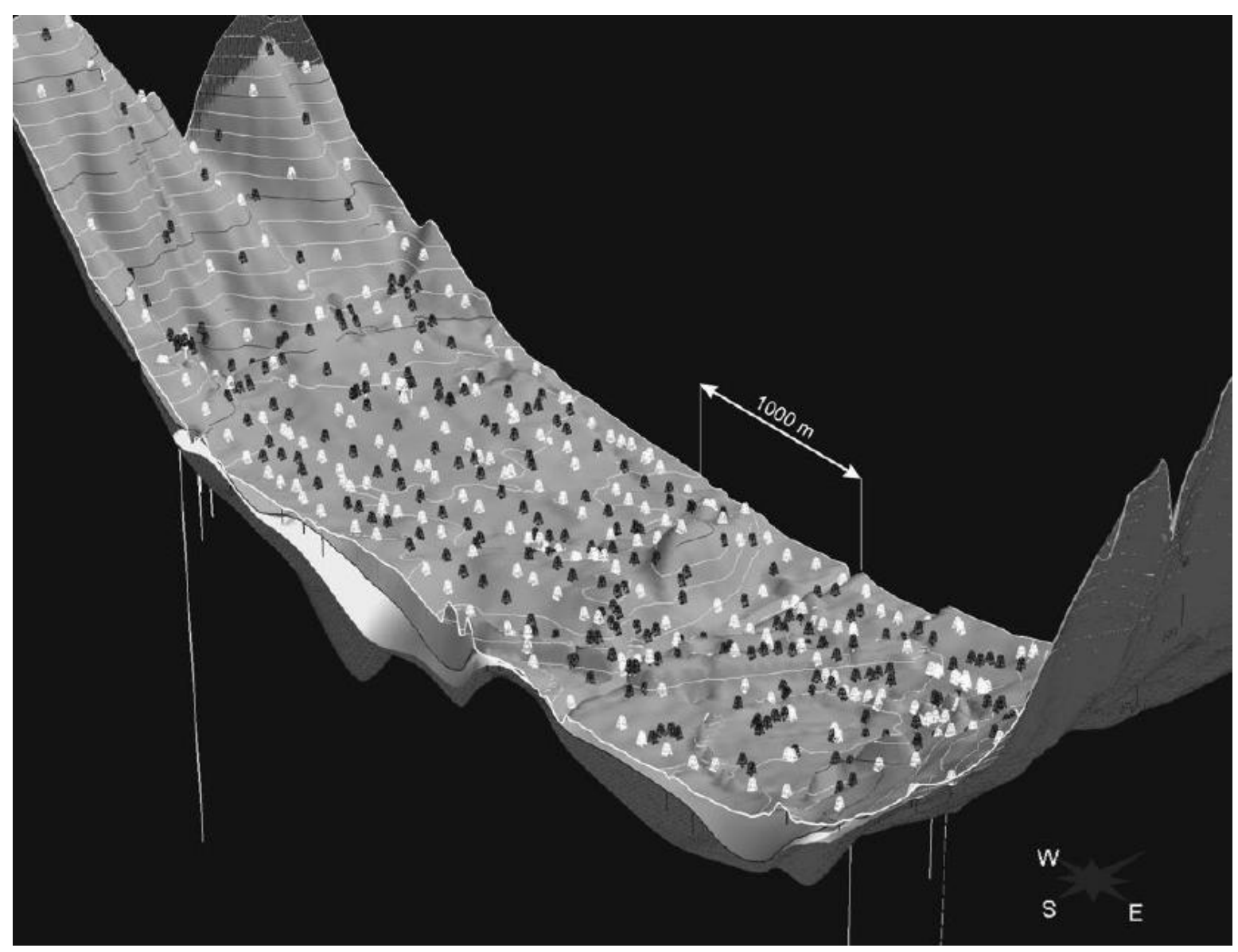

Figure 2.2 Southeast thee-dimensional view of the study area and the drilled boreholes locations applied in this modeling, with 20 times exaggeration in the vertical direction (Wagner et al. 2007).

Following, the procedure and the reasons of choosing the mentioned zone and layer have been elaborated:

\subsubsection{Choosing the layer unit 5}

Layer 5 (Pleistocene solifluction soils, river gravels and sand as well as fan deposits) was chosen to be the first layer to parameterize as it is besides local exceptions the base layer 
of the Göttingen model. The exceptions are local subrosion structures where also interstadial sediments with geotechnically critical characteristics were preserved. Because these structures are penetrated by only few boreholes, geostatistical modeling is not easy there (Wagner et al. 2007).

However, among the Layers 1 to 5, the Layer 5 is the most complex with respect to the grain size distribution which is one of the key parameters for geotechnical characterization and classification. Grain size bandwidth in the other layers is much narrower which is likely to result in geotechnically more homogeneous soil types. To evaluate the distribution of geotechnically relevant parameters in Layer 5 and to extract to some extent geological information at the same time, the German geotechnical soil classification standard of DIN 18196 was selected in a first approach for geotechnical parameterization. Since the Layer 5 was additionally separated into several subunits according to the local major sedimentation regimes, the Leine sub-basin in and around the inner city was selected as a training area, leaving areas with solifluction soils and low thickness on the hill slopes unconsidered (Wagner et al. 2007). Additionally, it is rather impossible to establish a proper correlation between those soils and the central basin part. For the same reason, too deep and narrow structures were excluded to increase the degree of lateral correlation (time/genesis), because they may have served as trapping structures for sediments of older ages.

Naturally the expectation was that the correlation among the points of the selected volume must be higher considering the genesis of the sediments. The improvements in the variograms observed after limiting to the mentioned layer, verified this expectation. The reason why focus of the study was drawn to the lowermost layer was that the upper layers were more homogenate where the fine materials were dominant. The other reason for focusing on the lowermost layer was the existence of the anthropogenic materials in the upper layers that make them too complicated to model and consider /find spatial variability structure to use geostatistical analysis.

\subsubsection{Grid transformation}

It is essential to consider that, most of the geostatistical methods require the re-definition of the coordinate space if the modification of the thickness and dipping of the sedimentary layers is significant within the extent of the model (Deutsch 2002, pp. 85- 
88). This considerable dipping or changes in the strikes of the geological structures may considerably decrease correlation in horizontal direction.

On the contrary, the transformation into a new coordinate system may not meet all the internal structure variations of a sedimentary body and may therefore lead locally to nonoptimal estimations. In fact this makes the appropriate selection of a coordinate transformation on the one hand a crucial step in the geostatistical modeling and on the other hand should be seen as the best compromise to deal with the whole sedimentary body (Deutsch 2002, pp. 85-88). The bigger volumes obviously, contain more data and are statistically more reliable. In crucial cases one would have to subdivide a volume again.

Figure 2.6 represents the Z-coordinate transformation with four different scenarios. In this study, the selected scenario for Z-coordinate system was according to case B of Figure 2.6 which has been suggested to be the best approximation for the lateral correlation of the sedimentary layers in such cases. According to this scenario in this study, to make a better stratigraphic continuity model among the relevant points (considering the genesis of the geological structures), the estimation grids were transformed into a new grid system proportional to the top and bottom surfaces of the Pleistocene layer. Such a grid is more appropriate for making a detailed threedimensional facies model (Deutsch 2002, p. 89). The reason is that the sedimentation, compaction, and consequent deformation of the sediments can cause a sort of proportional layering between the top and bottom surfaces. It has been considered that no significant erosion stage has been occurred and this inference makes sense due to the young age of the sediments. Therefore, it is anticipated that the stationarity assumption should become stronger after such transformation. A test on the variograms without transformation represented non-stationary-like and more vague variograms (see Figure 2.3). 

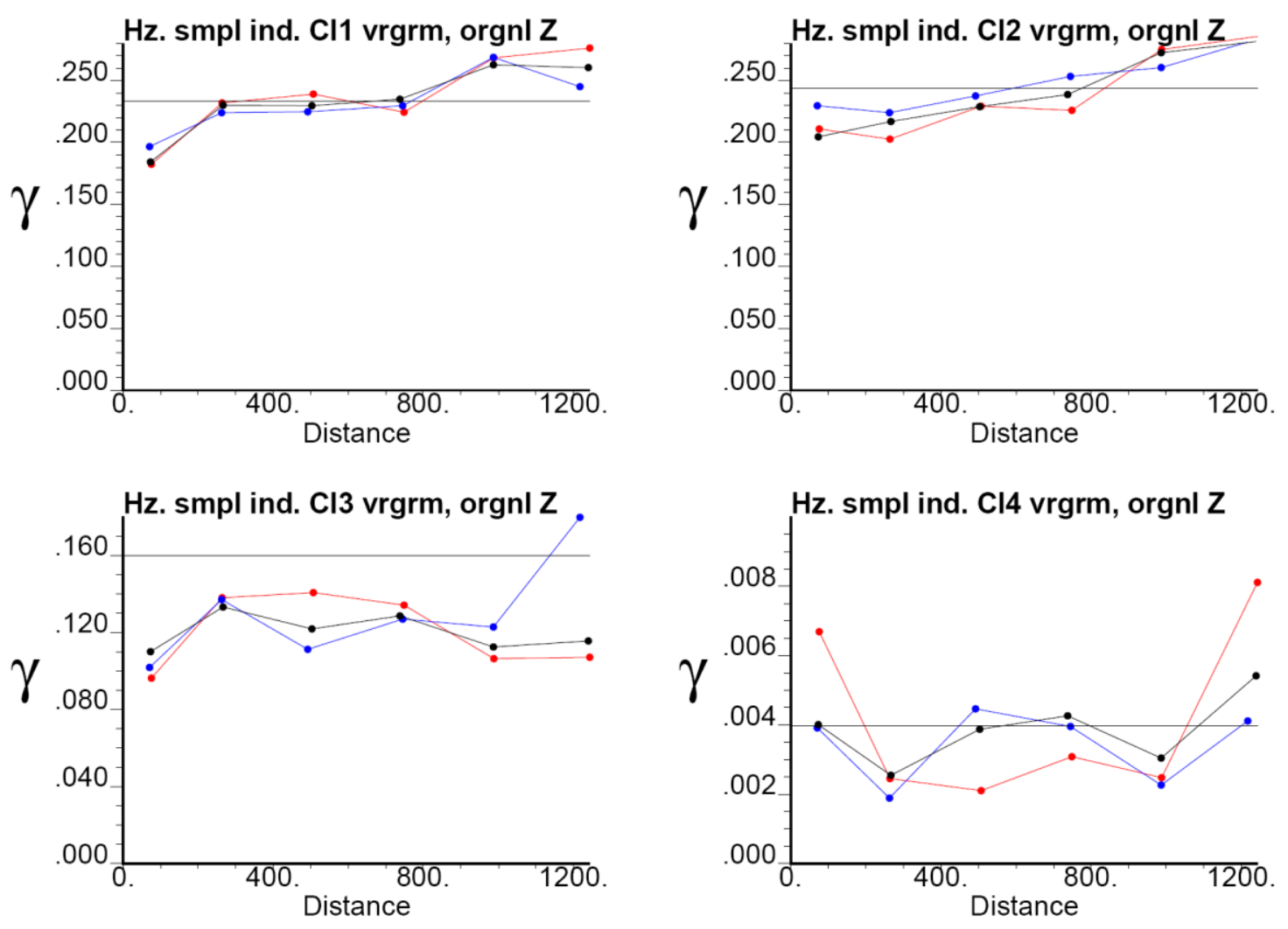

Figure 2.3 Horizontal sample variograms of the four geotechnical soil categories without vertical $(Z)$ co-ordinate transformation (i.e. in original $Z$ system), plotted in three directions; red representing the NS direction, blue representing the EW direction, and black representing the Omni-directional variogram. The horizontal solid black line represents the expected sills of each sample variogram.

Boreholes location map and mode cluster, eastern and western basins.
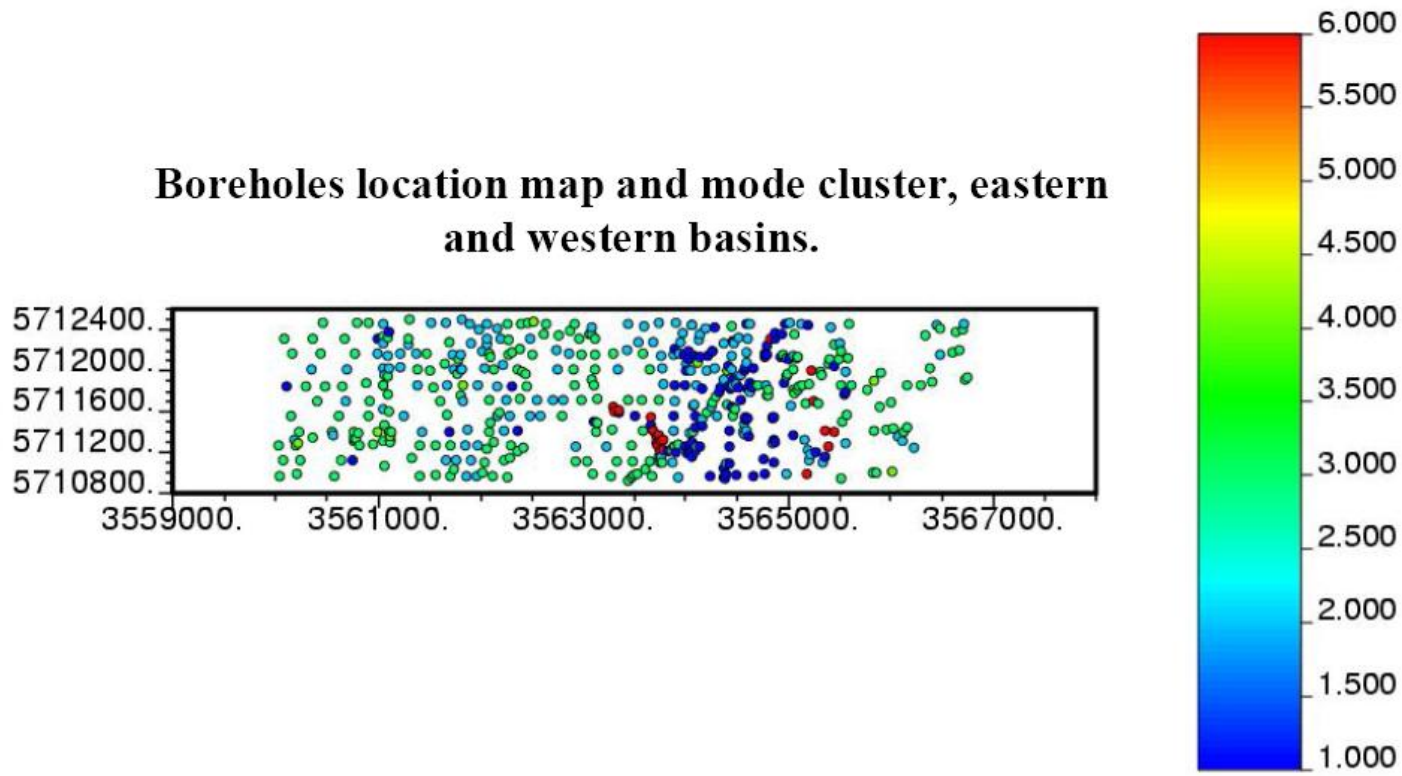

Figure 2.4 Location map representing the mode clusters (soil classes) of the Pleistocene layer in pilot zone in eastern and western basin of Göttingen soils project area (488 boreholes) excluding too deep samples (from the deep holes). Colors show the mode cluster (most observed) soil class in each borehole. 

basin.

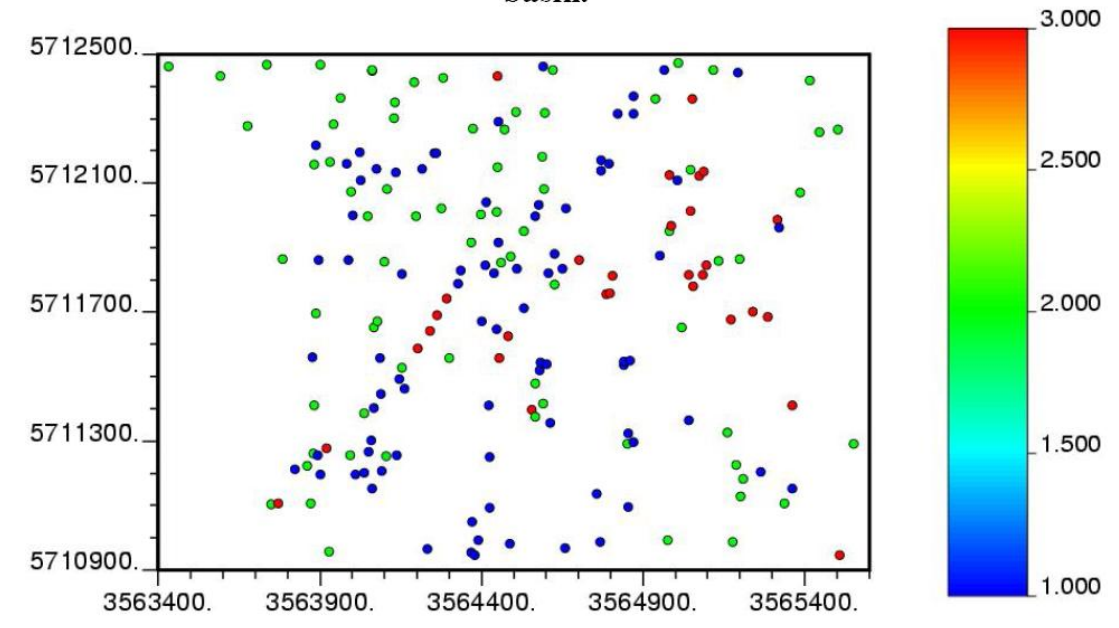

Figure 2.5 Location map representing the mode clusters (classes) of the Pleistocene layer in pilot zone in Eastern basin of Göttingen soils project area (188 boreholes) excluding too deep samples (from the deep holes). Colors show the mode cluster in each borehole.

As it is evident in Figure 2.3, they either show a constant increasing or straight trend of the variograms that exceed or fall more above or below the global estimated variogram sill based on the soil-class proportions $($ sill $=p(1-p)$ ) and in general much vaguer structure than those with the transformed grids (Figure 3.1). This phenomenon could also affect the results in the estimation and simulation stages in which the non-relevant or less relevant points could be accounted for estimating/simulating the unknown points in the IK, SISIM, or TP/MC methods, when no transformation is performed.

The grid transformation was performed on the borehole elevation $(\mathrm{Z})$ coordinate using the following equation (Deutsch 2002, p. 88):

$$
Z_{\text {relative }}=T_{\text {average }} \frac{\left(Z_{p}-Z_{\text {bottom }}\right)}{\left(Z_{\text {top }}-Z_{\text {bottom }}\right)}
$$

where $Z_{\text {relative }}$ is the transformed $Z$ (elevation) value for an initial point on the borehole (i.e. $\left.Z_{p}\right), \quad Z_{\text {top }}$ and $Z_{\text {bottom }}$ are the corresponding images of the mentioned point on the upper and lower surfaces of the bounding layer, respectively, and $T_{\text {average }}$ is the mean thickness of the Pleistocene layer in the study area. 

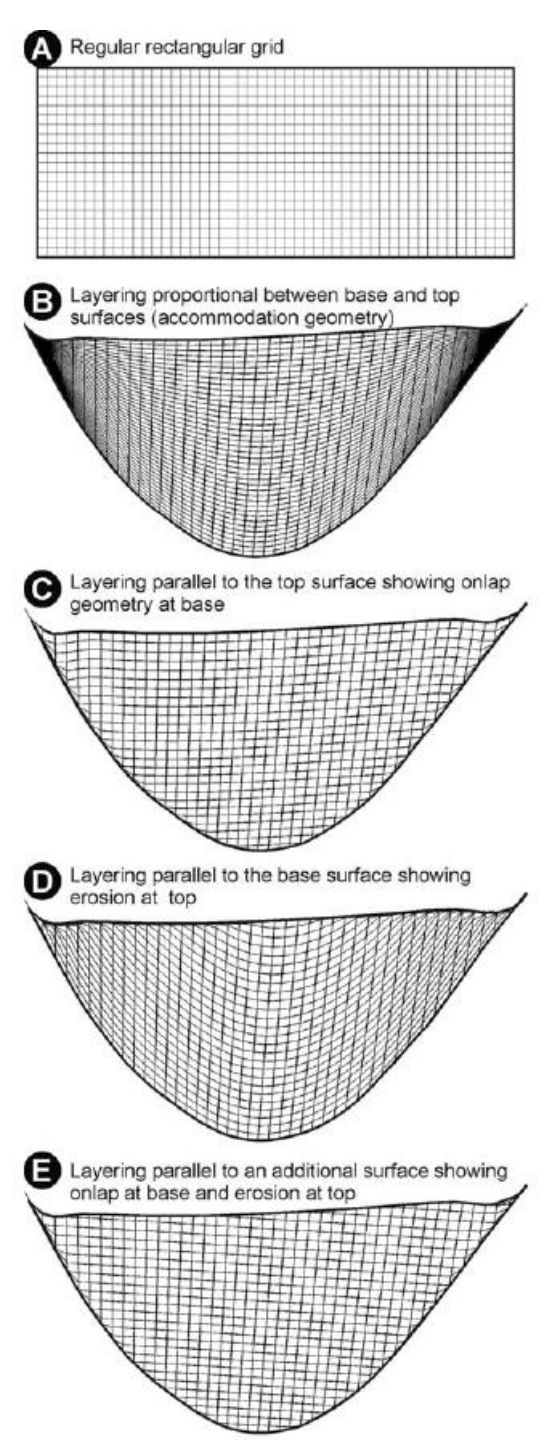

Figure 2.6 Various gridding systems for geostatistical modeling in different geological scenarios (Falivene et al. 2007, p. 203).

In order to make a grids transformation proportional to the top and bottom of the layer 5, the first step was to estimate the mean thickness of the layer over the investigation zone. Therefore, in the single boreholes, the thickness of the layer in each borehole was calculated as:

Thickness $=($ Top elevation of the layer $)-($ Bottom elevation the layer $)$

For the 188 boreholes, the arithmetic mean of the observed thickness of the study layer in each borehole was calculated and considered as an estimation of the mean thickness of the investigated layer, i.e. $T_{\text {average }}=6.7397 \mathrm{~m}$ (approximately). 
For any existing point then the grids transformation was performed according to equation $(2-1)$.

\subsubsection{Considered soil classes}

According to the classification scheme applied here, the soil classes 6 and 7 belong respectively to the artificial fillings and hard rock groups, and did not exist in the modeling zone. In addition, the soil class 5 that belongs to the group of organic materials, were absent in the selected zone (i.e. the eastern basin and layer 5). The observations of the soil class 4, which represents the freshwater limestone or sediments with some organic constituents, were very limited and isolated. In addition, their samples are more prone to the uncertainties due to some reasons that are explained briefly here. It is probable that some sediment was brought from the upper layers to the lower layers during the drilling process because the materials were unconsolidated. In addition, the logrecorder staffs might have made some mistakes in the interpretations of the samples, for example magnesium oxides that have black appearance might have been mistaken with the organic materials.

Because the boundaries of the layers were drawn based on; available samples, geological evidences, and interpretations, it is also possible that the interpretation of the boundaries were not completely exact or they maybe not absolutely sharp. Therefore, some sediment, especially near the boundaries of the layers that might belong to another layer, might be wrongly considered in the other layer.

\subsubsection{Separating the slope sides of the basin}

According to the geological interpretations, the solifluction soils can be found just in the upper basin sides where the sediments were entered into the basin. The soilfluction soils are the soils or materials found in "the slow downhill movement of soil or other material in areas typically underlain by frozen ground" ("Solifluction." The Free Dictionary. Farlex, 2009 ${ }^{1}$ ). Therefore, these sediments do not belong to the channel sediments. Figure 2.7 illustrates the solifluction phenomenon in a schematic section.

\footnotetext{
${ }^{1}$ The Free Dictionary. Farlex, 2009. Web. Winter 2011. <http://www.thefreedictionary.com/solifluction>. Originally has been taken from: The
} 


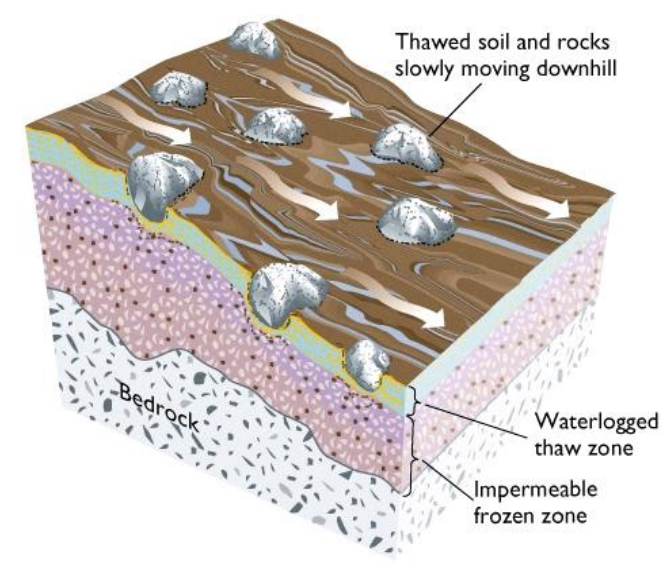

Figure 2.7 A schematic illustration of solifluction materials, their location, and formation (Solifluction [Solifluction or Frost Creep (example)]. Copyright 2000-2001. Photograph (Image). Index of Teacher, Geology 12, Photos, Belmont Secondary School. Web.).

On the other hand, due to choosing a relative grid system in the vertical direction, the density of the grids would become too high in the slope sides, which does not seem so reasonable. Therefore, due to the lower sampling rates in the basin sides and to prevent mixing the geologically different pieces of the model with on another, it was decided to eliminate the basin sides from this model and do the modeling for them, separately.

\subsubsection{Choosing the eastern basin}

As mentioned before, the test site was limited to the eastern basin or in other words to the eastern part of the primarily selected rectangular zone (

Figure 2.5).

There were mainly two reasons for doing so; first, getting a better stationarity with considering a more relevant and similar points in a modeling run because the conditions of the eastern and western basins are certainly different, and second, dealing with a higher heterogeneity in the eastern basin (with a higher basin energy during the sedimentation) created the sedimentary patterns during the deposition. Therefore, the eastern basin with a much higher heterogeneity needed a more precise modeling and evaluation.

American Heritage ${ }^{\circledR}$ Dictionary of the English Language, Fourth Edition copyright (C2000 by Houghton Mifflin Company. Updated in 2009. Published by Houghton Mifflin Company 


\subsubsection{Summary statistics of the data-sets in Göttingen test site}

The aim of this section is to evaluate the main features and behaviors of the existing datasets. The general statistical evaluations can be discussed in two main sections; univariate, and bivariate statistics.

Table 2.1 Summary statistics of the main two-dimensional parameters.

\begin{tabular}{|c|c|c|c|c|c|}
\hline Ptatistics & $\mathbf{X}$ & $\mathbf{Y}$ & Top & Bottom & Thickness \\
\hline Min. $^{*}$ & 3563590 & 5710945 & 140.2 & 127.2 & 0.2585 \\
\hline 1st Qu. $^{* *}$ & 3564095 & 5711369 & 142.9 & 134.6 & 3.8632 \\
\hline Median $^{*} 3564455$ & 5711818 & 144.0 & 137.6 & 6.6319 \\
\hline Mean $^{\text {Mrd Qu. }}{ }^{* *}$ & 3564510 & 5711754 & 145.2 & 138.4 & 6.7397 \\
\hline Max. & 3564864 & 5712137 & 145.9 & 141.3 & 9.3086 \\
\hline
\end{tabular}

* Minimum., ** First quartile, $* * *$ Third quartile. *** Maximum.

\section{(1) Univariate statistics of the data-sets in Göttingen test site:}

In this section, first of all, there is a general glance at the statistics of the main twodimensional parameters of the borehole data in the study zone, including $\mathrm{X}, \mathrm{Y}$, Top (elevation of the top of the layer 5 observed in the boreholes), Bottom (elevation of the bottom of the layer 5 observed in the boreholes), and Thickness (of the study layer). Table 2.1 summarizes these statistics.

One of the parameters that can be inferred from this table is the average thickness of the layer which is used in the grid transformation stage. Another important univariate statistic for the three-dimensional modeling of the soil classes in this study, especially for the geostatistical simulations, is a representative histogram of the soil classes or the proportions of each soil class. 


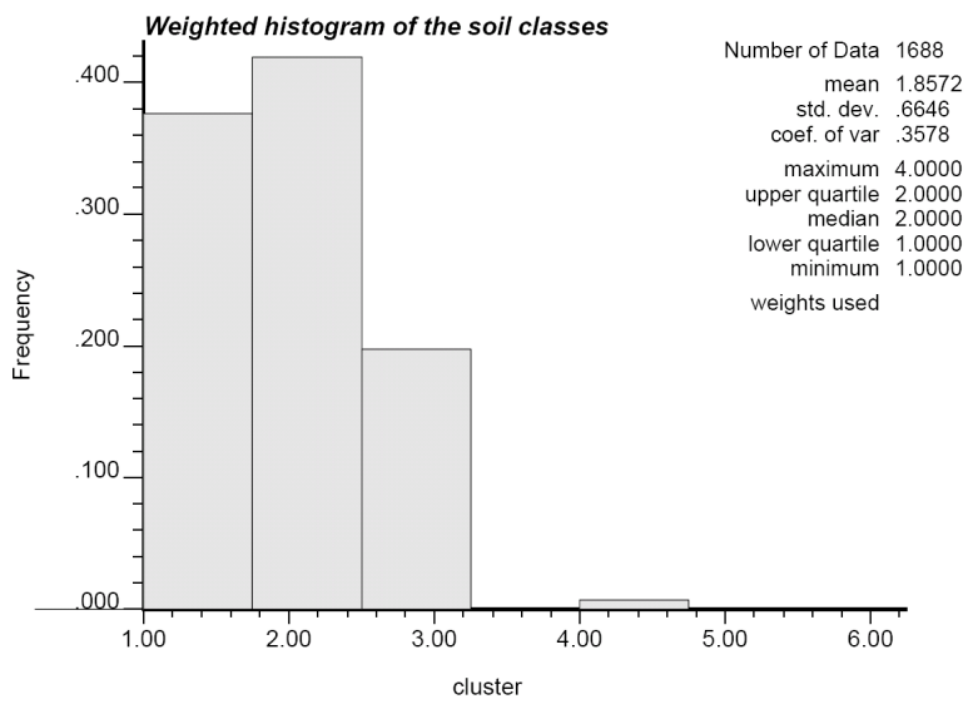

Figure 2.8 The weighted histogram of the soil classes derived from the input data after de-clustering

This histogram should be a true representative of the volumetric proportions of the soil classes in the study zone. Actually, the simple histogram of the soil-class data from the boreholes does not reflect a proper estimation of the real volumetric proportions of the soil classes and needs some corrections.

In addition to the fact that the sampling is not exhaustive, two other factors can mainly affect the histogram of the borehole data to become non-representative; first, a clustered distribution of the boreholes over the study zone, and second, the grid-transformation before the modeling course. In spite of the fact that the clustering of the boreholes in this case was not too high, the effect of the change in the support of the samples in the vertical direction (the representing volumes of the samples) after grid transformation was significant and could also be considered with declustering algorithms like celldeclustering method. More details about the declustering are explained in Deutsch 2002, pages 50 to 63 . The only problem with the cell-declustering method is that, it depends considerably on declustering parameters defined in the algorithm and can produce various results with various declustering parameters. Once with an optimum selection of the celldeclustering parameters, and once with only modifications related to the vertical transformation of the modeling grid (for correcting the representing volumes of the sample cells), the proportions of the soil classes were calculated and compared together. The results were very similar. As mentioned before, because of the lack of a considerable 
clustering of the boreholes, the estimation of the proportions by only vertical gridtransformation corrections, should have given a proper estimation of the soil-classes proportions. This inference was verified by matching the results produced by celldeclustering and calculating the proportions only with corrections for vertical transformations.

Figure 2.8 illustrates the borehole samples histogram after declustering. Table 2.2 summarizes the frequencies and proportions of the soil classes achieved from compromising the results from the two mentioned methods.

Table 2.2 Representative frequencies and proportions of the soil classes based on the observations in the boreholes with modifications using declustering and considering the representing volumes of each sample after grid transformation

\begin{tabular}{|c|c|c|}
\hline Soil classes & Frequencies & Proportions (p) \\
\hline Cl1 & 627.790 & 0.372 \\
\hline Cl2 & 714.020 & 0.423 \\
\hline Cl3 & 339.29 & 0.201 \\
\hline Cl4 & 6.750 & 0.004 \\
\hline
\end{tabular}

\section{(2) Bivariate statistics of the data-sets in Göttingen test site:}

In this section, the aim was to assess the dependence between the pairs of the parameters and their changes when another one is changing. In cases, these studies can be applied to get a feeling of a probably existing trend in the data-set over the study area as well.

Scatter-plots of the soil classes (clusters) versus the $\mathrm{X}, \mathrm{Y}$, and $\mathrm{Z}_{\text {relative }}$ coordinates, show the distribution of different soil classes in various parts of the study site.

Scatter-plot of the soil clusters along the X-coordinate represents that in general there were no considerable preferential distribution of the clusters across different $\mathrm{X}$-coordinate zones (Figure 2.9). Soil classes 1 and 3, tend to occur more in the middle of the basin. Soil class 2, was distributed nearly all-over the site and soil class 4 was observed around few points in the middle. The distribution of the soil classes across the Y-coordinate was even more homogeneous and covered nearly the full range of Y changes (Figure 2.10). Class 4, was again observed around few points slightly tending to be less isolated to the North. The distribution of the soil classes was even more monotonous relative to the 
$\mathrm{Z}_{\text {relative }}$ coordinate. Only the few observations of the class 4 had a slight tendency to occur in the more superficial parts (Figure 2.11). These scatter-plots verified, in general, the lack of a considerable trend across the coordinates of the study zone. Though, the indicator variograms also can reflect the existence of an important trends when exist. As discussed in the next sections, the indicator variograms also did not reflect a significant trend in transformed grid in this survey.

It should be considered that the evaluated behavior of the categorical variables in the scatter-plots can not be assessed by the common methods applied for the continuous variables. Yet, some similarities could be suggested in the evaluations of these two data types.

About the other factors (continuous parameters), as expected, the top and bottom surface elevations of the layer were strongly (i.e. R>0.8) correlated (Figure 2.12). Naturally, it means that the top surface goes higher when the bottom surface does so and vice versa. The bottom elevation is also strongly but negatively correlated $(\mathrm{R}<-0.8)$ with the thickness of the layer (Figure 2.14). These strong correlation was significant even with a very low significance level such as $(\alpha=0.0001)$ due to the rather large number of observations. It means that, the layer is thicker where the layer is deeper.

Although the top elevation also had a negative correlation with the thickness of the layer, this negative correlation was weak while significant even with very low significance levels like ( $\alpha=0.0001$ ). Hence, the layer is thicker wherever the (top) surface of the layer goes higher and vice versa.

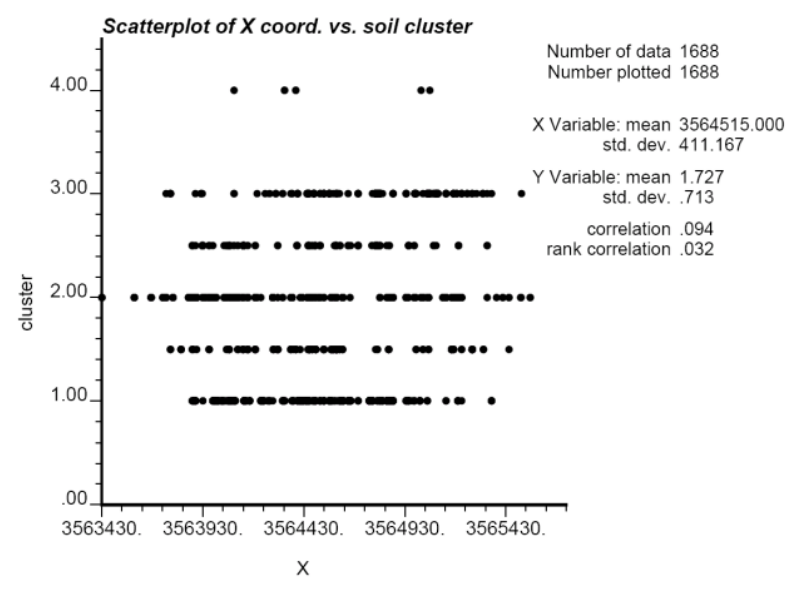

Figure 2.9 Scatter-plot representing the occurrence of soil clusters along the $\mathrm{X}$-coordinate. 


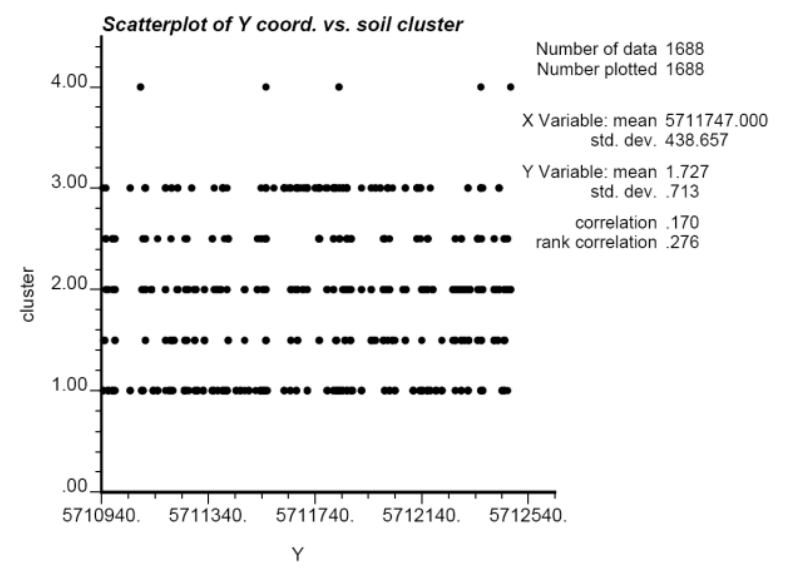

Figure 2.10 Scatter-plot representing the occurrence of soil clusters along the Y-coordinate.

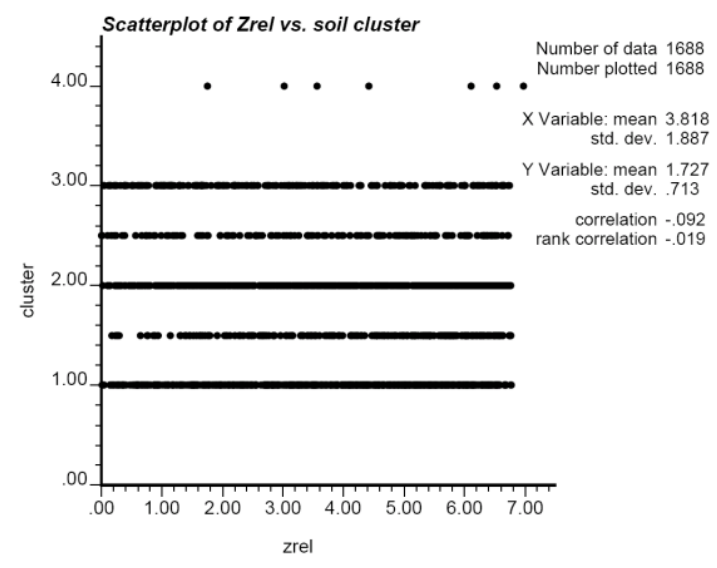

Figure 2.11 Scatter-plot representing the occurrence of soil clusters along the $Z_{\text {relative-coordinate. }}$

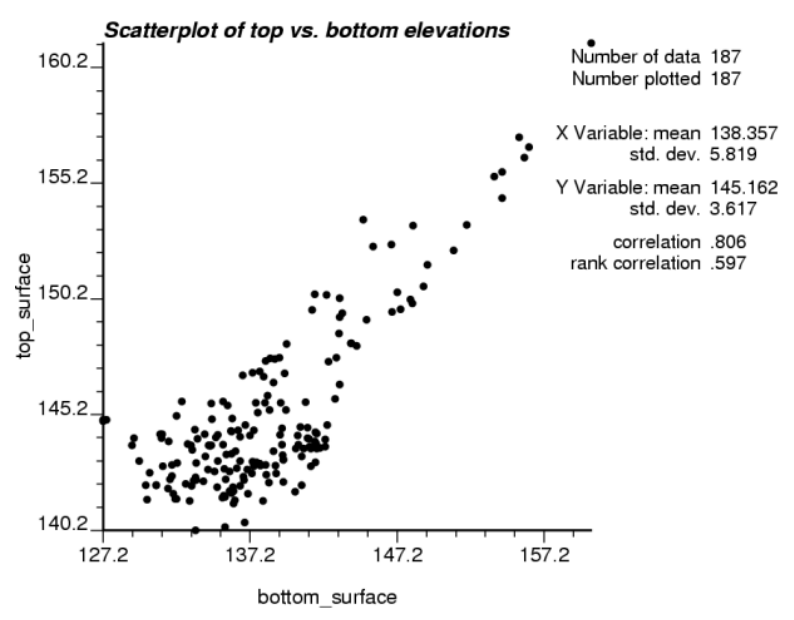

Figure 2.12 Scatter-plot of the top versus bottom elevation surfaces elevations of the study layer. 


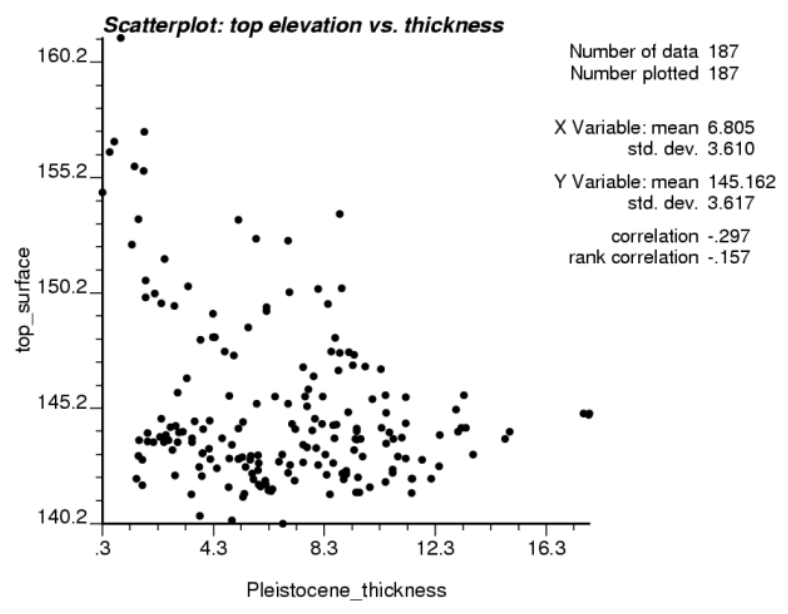

Figure 2.13 Scatter-plot of the top elevation versus Pleistocene layer thickness (the study layer).

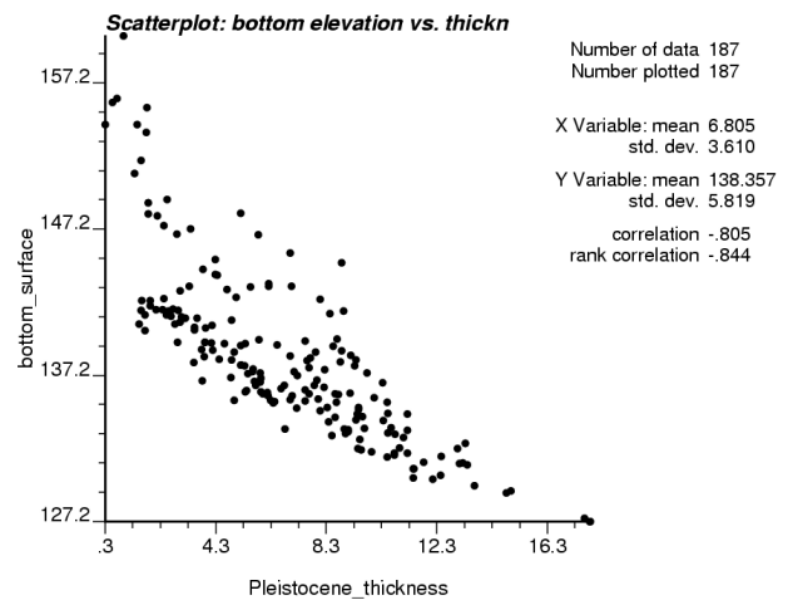

Figure 2.14 -plot of the bottom elevation versus Pleistocene layer thickness (the study layer).

The determination of the significance level of a Pearson correlation coefficient has been explained in many statistical resources and textbooks (e.g. McKillup and Darby Dyar 2010; Davis 2002). 


\section{Indicator kriging (IK) analysis in the Göttingen test site}

\subsection{Overview}

As explained before, the indicator kriging (IK) can be applied to estimate the existence probability of every available soil or facies category in an unknown point of the model. To do so, the indicator variables were assigned at the sample points for each soil class. Hence, the indicator value of a soil class which exists at a sample point, were equal to one (i.e. $\mathrm{I}=1$ ) when that category existed at the sample point and zero (i.e. $\mathrm{I}=0$ ) otherwise. These indicator values in the transformed grid system (see Section 2.1.2) were applied to calculate the sample indicator variograms of each category and fit the proper analytical models to them. The variogram models were then evaluated and improved using crossvalidation and jackknife criteria. The fine-tuned models were then employed to do IK estimations for each soil class. The outcomes of these estimations were the probabilities of the existence of each soil category at each estimation point. To achieve the final IK model, the soil category with the highest estimated probability of existence (obtained by IK estimation) was assigned to each estimation point. A simple MATLAB code written by the author of this thesis was applied for this purpose. The final soil-classes model was evaluated statistically/geostatistically for histogram- and indicator variogramsreproduction and geologically for its matching to the geological interpretations. Finally, the estimation results were brought back into the original gird system and represented by gocad software.

\subsection{Variograms and spatial variability modeling}

\subsubsection{Introduction}

As stated previously, after assigning the indicator values $\left(\mathrm{I}_{\mathrm{k}}\right)$ to all sampling points for each geotechnical soil class $\mathrm{k}$, the first step in geostatistical analysis was to calculate the indicator variograms of each soil class and in different directions. Figure 3.1 represents the sample indicator variograms of the soil categories in various directions and their fitted models. The main directions considered for calculating the variograms were North-South 
(N-S), Northeast-Southwest (NE-SW), East-West (E-W), Southeast-Northeast (SE-NW), and Omni-directional, for horizontal directions $(0,45,90,135$, and all-directions azimuth and tolerance), as well as the vertical direction. The sample horizontal variograms do not prove any obvious horizontal anisotropy. This phenomenon can be due to the lack of sufficient data in the horizontal direction to infer any probably existing horizontal anisotropy. Therefore, the existence or lack of any anisotropy in the horizontal direction could not completely be verified or rejected in this case. Due to the slight differences among the sample variograms in horizontal directions and lack of clear significant detectable horizontal anisotropies in them, together with the lack of a certain and clear evidence for such anisotropy direction based on the geological interpretations, they were modeled by isotropic models in horizontal direction.

Obviously, in the variograms according to the horizontal and vertical variogram graphs, a significant zonal anisotropy is evident. The horizontal variograms have a nugget effect while the vertical ones either do not, or have only very little ones, and the sills and ranges are also different between the horizontal and vertical directions. For modeling such a zonal anisotropy in the indicator variogram of a soil class, an appropriate solution, could be trying to reflect the nugget effect of the corresponding horizontal variograms on the sill of each vertical variogram. The reason for doing so is that, the vertical variograms reflect the spatial variability in short distances whereas the horizontal ones reflect the long-distance spatial variations. The prospect is that the indicator variograms should not reach their expected sills fully in vertical directions (due to the incomplete coverage of the data variations in their plotting ranges) while they should reach their expected sill in the horizontal directions that have a much bigger coverage of the data variations. The evaluation of the characteristics of the horizontal and vertical indicator variograms confirms these expectations. Therefore, as an initial check, the expected sills for the indicator variogram of each soil category were calculated having their global proportions. The expected sills for the mentioned indicator variograms of each soil category were calculated by:

$$
c=p(1-p)
$$

in which $c$ stands for the expected sill, and $p$ is the proportion of the corresponding soil category. 

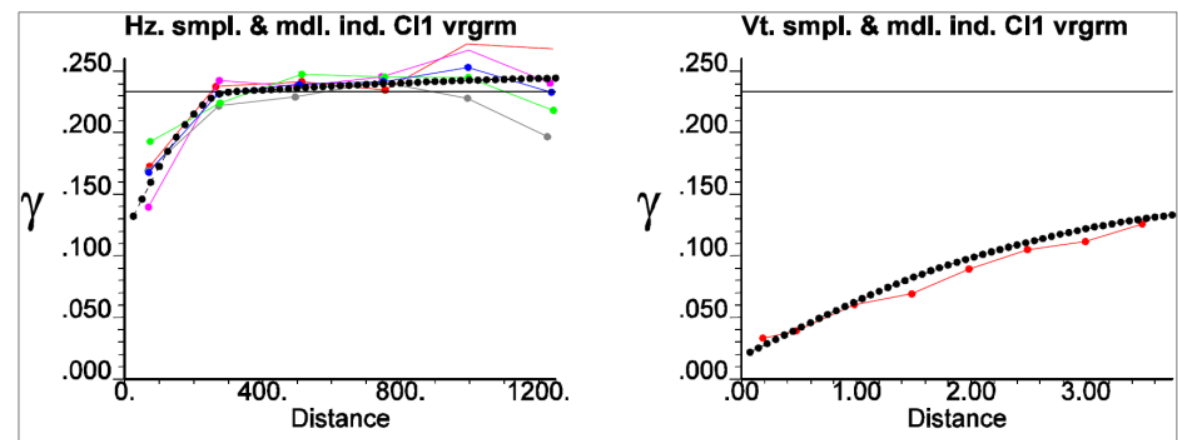

Hz. smpl. \& mal. ind. $\mathrm{Cl} 2$ vrgrm

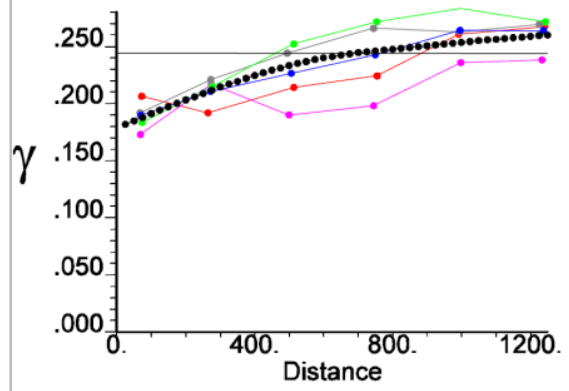

Vt. smpl. \& mal. ind. $\mathrm{Cl} 2$ vrgrm

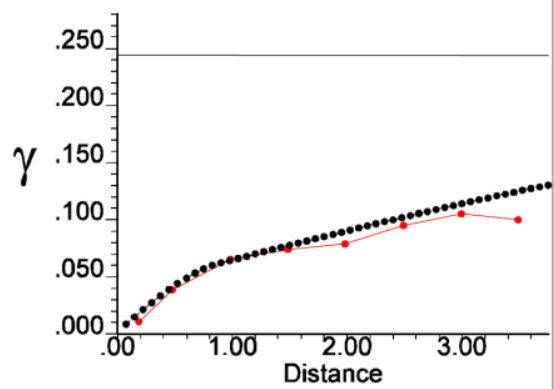

Hz. smpl. \& mdl. ind. $\mathrm{Cl} 3$ vrgrm

Vt. smpl. \& mdl. ind. $\mathrm{Cl} 3$ vrgrm
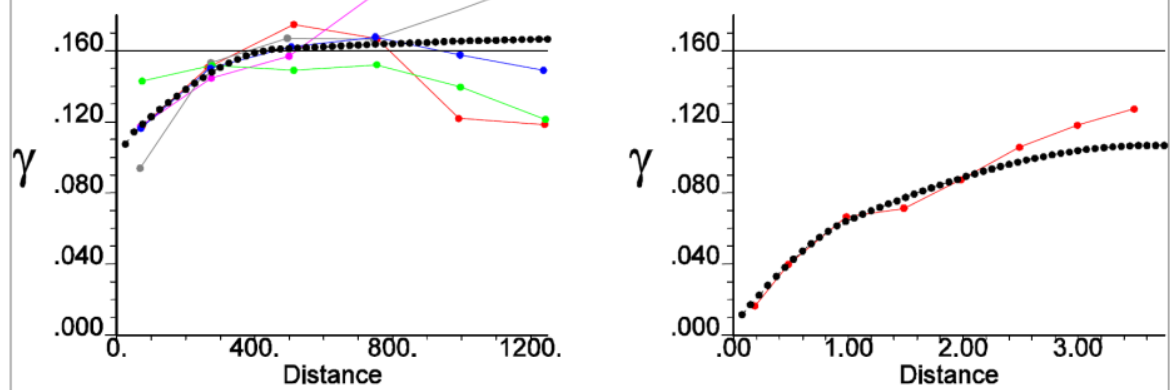

Hz. smpl. \& mdl. ind. Cl4 vrgrm

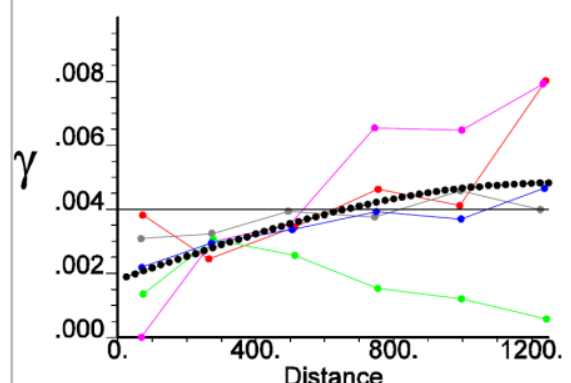

Vt. smpl. \& mdl. ind. Cl4 vrgrm

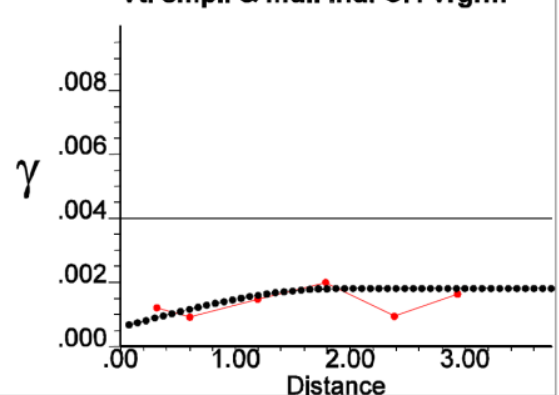

Figure 3.1 Experimental and model indicator variograms of geotechnical soil classes 1 to 4, in horizontal and vertical directions (left-side and right-side graphs, respectively). Red, purple, green, gray, and blue solid lines in horizontal variograms represent N-S, NE-SW, E-W, SE-NW, and Omnidirectional variograms, respectively. In the vertical variograms, red lines represent the sample vertical variograms. The black dashed lines show the model variograms for both vertical and horizontal variograms.

The model variograms which have been obtained from the combination of the horizontal and vertical model variograms sometimes go beyond the theoretical sill. However, in their effective ranges, they are very well in accordance with the experimental variograms and expected sills. For making the variography clearer, the Omni-directional variograms also 
have been represented in Figure 3.2, which has been used as the basis for building the three-dimensional models since the horizontal variograms were modeled isotropically.

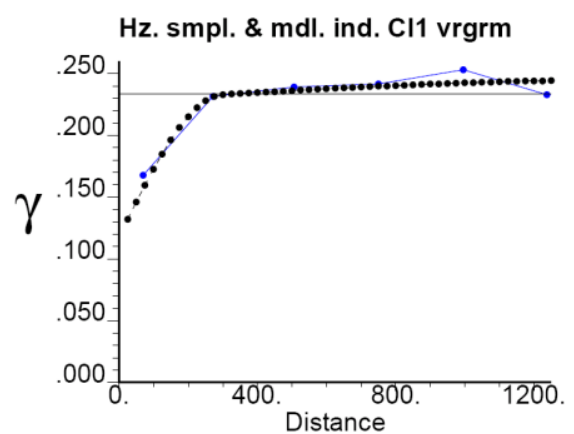

Hz. smpl. \& mdl. ind. $\mathrm{Cl} 2$ vrgrm

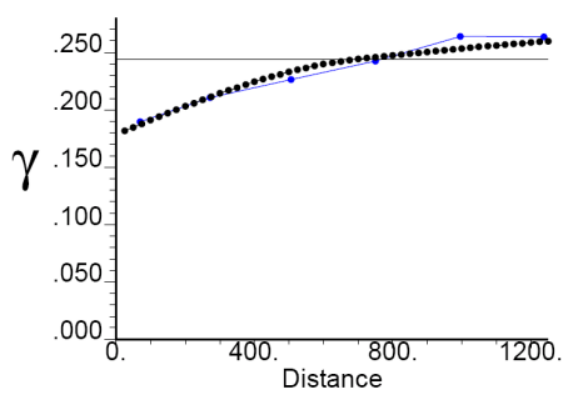

Hz. smpl. \& mdl. ind. Cl3 vrgrm

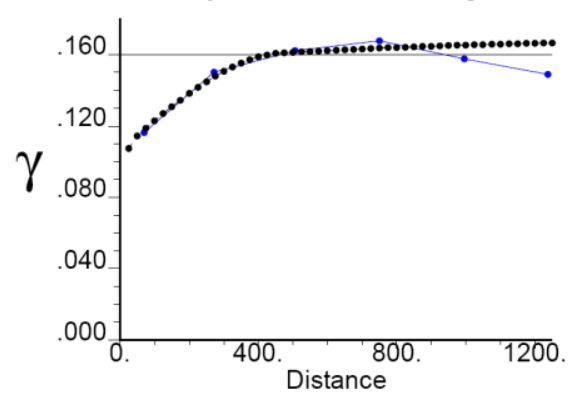

Hz. smpl. \& mdl. ind. Cl4 vrgrm

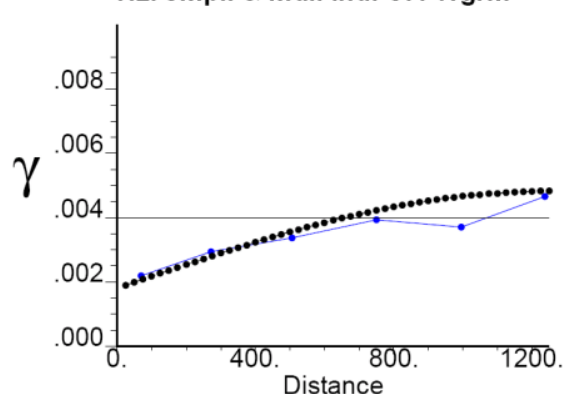

Vt. smpl. \& mdl. ind. Cl1 vrgrm

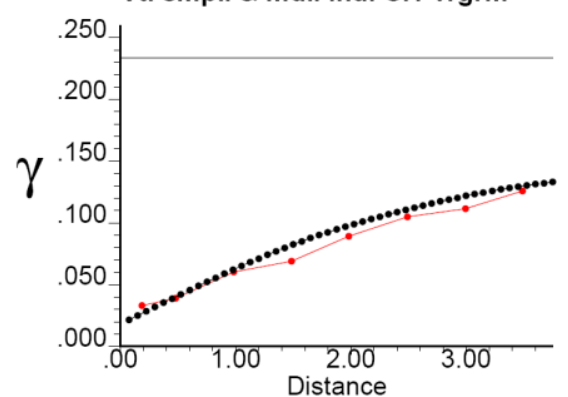

Vt. smpl. \& mdl. ind. $\mathrm{Cl} 2$ vrgrm

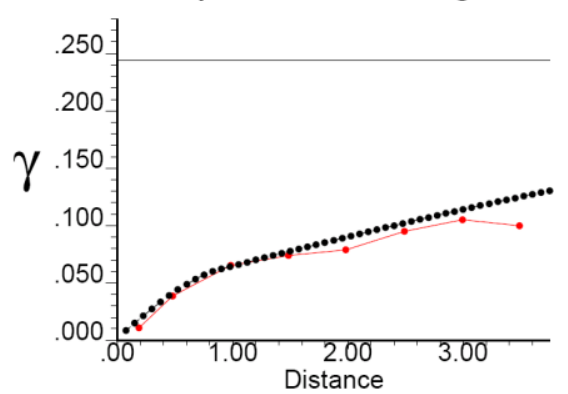

Vt. smpl. \& mdl. ind. Cl3 vrgrm

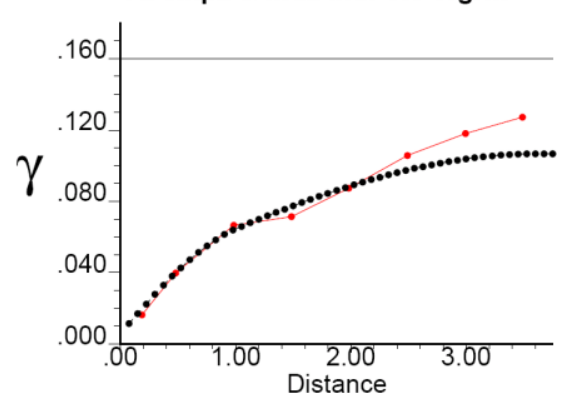

Vt. smpl. \& mdl. ind. Cl4 vrgrm

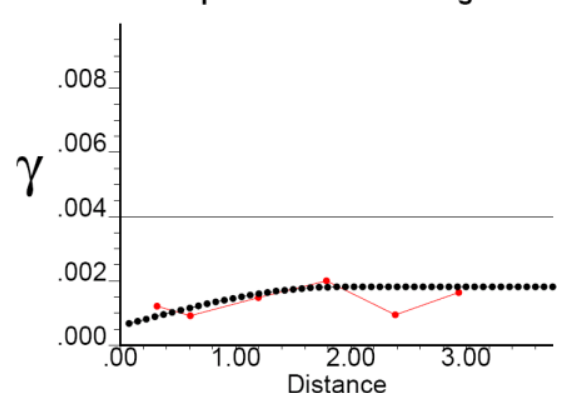

Figure 3.2 Experimental and model indicator variograms of geotechnical soil classes 1 to 4 , in horizontal and vertical directions (left-side and right-side graphs, respectively). Blue solid lines in horizontal variograms represent Omni-directional variograms. In the vertical variograms, red lines represent the sample vertical variograms. The black solid lines show the model variograms for both vertical and horizontal variograms. 


\subsubsection{The place of interpretations in variogram modeling}

Variogram (or more specifically indicator variogram in this case) is one of the most essential bases in geostatistical modeling practice. This crucial importance is not only for the reason that variogram serves as a spatial variability analysis and quantification means but also its analytical model is an important perquisite for the majority of the geostatistical estimation and simulation methods (Gringarten and Deutsch 2001,p. 507). When talking about the variogram modeling, the aim is to find an optimal analytical variogram model which is a permissible model that at the same time conforms to the observations and any ancillary information about the phenomenon (Goovaerts 1997, p. 97).

The requirement of the application of analytical variogram models as well as the meaning and necessity of the permissibility of the variogram models have already been explained from page 26 (part ((f)) from section 1.4.2).

By choosing the known permissible variogram models or their combinations, the permissibility condition of the variogram model is guaranteed. For the second prerequisite, i.e. their matching with the observations and other types of available information, there are two sources; available data-sets, and interpretations (or the experts' knowledge and opinions).

To ensure that the variograms conform to the attribute data, one can try to fit a variogram model with the least deviation of the model from sample variogram values. In this regard, more stress should be given to the sample variogram values calculated with a more numbers of point-pairs. The sample variogram points near the origin which reflect the main shape and slope near the origin as well as the nugget-effect of the variogram are also very essential (Chilès and Delfiner 1999, p. 104 , Armstrong 1998, p. 53, and Morgan 2011, p. 61). However, there is still a big uncertainty in variogram modeling. For instance, a single sample variogram can be modeled with a range of analytical models and with different parameters in each.

Another approach to evaluate the quality of the variogram models and possibly improve and optimize them is to implement the cross-validation technique. The variogram modeling is rarely a final goal in geostatistical analyses. The ultimate objective is usually to do optimal and precise estimations and simulations of the attributes such as the existing soil type in a point or the porosity of a layer. More details about the cross-validation 
method will be discussed later. In fact, in cross-validation, the attempt is to optimize the variogram and estimation models to obtain more precise estimations (Goovaerts 1997, p. 105). However, the data from samples can rarely reflect sufficiently the existing geological, structural, and other facts about the phenomenon under study. For the models of this study to become more consistent with the existing and plausible three-dimensional geological continuities, the geological interpretations also were deemed. In other words, the linkage between the geology and the variogram behavior should be understood very well before and during the variogram modeling. For example, about choosing a suitable variogram model that conforms to the reality, the following points can considered:

(a) The nugget effect: is related either to the short-scale variations or the uncertainty and measurement errors. The quantity of the nugget effect should be decided according to the nature of measurements and the expected short-scale variations. The higher the short-range variations or measurement errors, the bigger a nugget effect should be considered in the variogram model (Gringarten et al. 2001).

(b) The hole-effect model and periodicity: undulations in a sample variogram can be a sign of cyclic structures and periodicity. A question may be raised in this case: according to the existing evidences from the reality or according to what the expert believes, is the periodic structures really expected about the variable which is being modeled? The existence of a periodicity should be confirmed by interpretations prior to decision of fitting a hole-effect model to such variograms (Gringarten et al. 2001).

(c) Anisotropies: a geometric anisotropy is reflected in the medium-scale variances with different variogram ranges in diverse directions. When non-isotropic features, for example elongations in specific directions are expected, the geometric anisotropy can be verified. Specially, in the case of insufficient observations that reflect a clear anisotropy in the sample variogram, a priori knowledge about the phenomenon and its geology can be helpful for deciding about the geometric anisotropies. In large-scale variances, when a variogram does not reach its full theoretical sill, a zonal anisotropy is speculated. A full variability range observation of the attribute is not expected in the corresponding direction of the mentioned variogram in such cases. The geological interpretations should verify the zonal anisotropy when fitting such anisotropic models (Gringarten et al. 2001). 
More discussions and details about the guidelines of the three-dimensional sample variogram interpretation and modeling are elaborated in Gringarten et al. 2001.

In the case of this study, the structures of the sample variograms and their interpretations were discussed in the project team to verify the geological soundness and agreement with the expected patterns prior to and after modeling the sample variograms. Hence, the variogram models were confirmed for their accordance to the geological interpretations (Gringarten et al. 2001, p. 526).

\subsubsection{The validation of the indicator variogram models}

Before any estimation and simulation stage, the variogram models should be evaluated, validated, and in the necessity case, should be improved.

To ensure that the variogram models being applied in the estimation and simulation stages are reliable and appropriate, the variogram models have to be validated first. The validation of the variogram models was done in this study using the two validation methods of; cross-validation, and jackknifing to evaluate the impact of using different variogram models or variogram parameters on the estimation models.

When using the cross-validation and jackknifing methods, it should be considered that, the cross-validation method is not necessarily sufficient to decide whether or not a variogram model and estimation parameters are optimal in modeling or estimation problems (Goovaerts 1997, pp. 105-106). For example, the re-scaling of variogram models does not affect the kriging weights and hence cross-validation of variogram sills is not possible by this method. More details about such shortcomings of the cross-validation method have been discussed in Goovaerts 1997 (pp. 105-106).

\section{(a) cross-validation:}

In cross-validation which is a "leave-one-out" technique, each sample (with the known variable) is omitted once and its value is estimated using the rest of the observations with different variogram models or various model parameters. This procedure is conducted for all the available samples. Cross-validation can be applied to assess the goodness of the variogram models for the subsequent estimation stages (Goovaerts 1997, pp. 105-106). Cross-validation was also applied here to verify the quality of the variogram models 
employed in geostatistical simulations. After conducting cross-validation using the GSLIB software, the estimated and true (known) values in the entire sample locations were compared together using a number of different tools. For example, in the continuous variables, creating the scatter-plot of the true versus estimated values is a common way to evaluate the results of a cross-validation. In the case of using the true versus estimated cross-validation scatter-plot, the points of the plot should ideally fall on the 45-degree line crossing from the origin of the coordinates, ideally the correlation-coefficient should be one and the averages of the estimated and true values should be the same. In practice, the ideal conditions are almost never met. However, the closer the scatter-plot, correlation coefficient, and the variable means to the ideal case, the better the quality of the models. This test were done for different models, different model parameters, and even for different estimation parameters such as the search radius and so on, to achieve the best estimation models and estimation parameters.

Another approach to evaluate the cross-validation results of the variograms/estimation models is to plot the histogram of the errors. The error is defined here, as the difference between the true and estimation values:

$$
\text { Error }=\text { true-estimate }
$$

This histogram should ideally represent a very narrow, symmetric, and zero-centered shape (i.e. the mean of the errors equals to zero), that reflect the lack of a significant and systematic error in the estimations.

In the categorical data-sets such as the facies or soil types, the cross-validation method is somehow different. An alternative to cross-validation of the indicator variogram models for the indicator kriging method could be the cross-validation of the sample indicators $\left(\mathrm{I}_{\mathrm{k}}\right)$ of any soil class (k) versus the estimated probabilities from the indicator kriging estimations. In this case, the estimated probabilities of the soil classes form the indicator kriging method is compared to those of the original data which are 1 where a soil class exists, 0.5 where it was suspicious to belong either to the mentioned soil class or to another one (unsure case), and 0 when that class did not exist at that location. This criterion can be evaluated using a scatter-plot of the predicted (using the indicator kriging technique) versus the actual probabilities (from data). Then, the distribution of the scatterplot points around the 45-degree line could be assessed using a similar framework to that explained for the continuous variables (Deutsch 2002, pp. 304-308). However, this approach contains an essential drawback in the estimation of the categorical variables because the final goal in the indicator kriging of the categorical variables is not to 
estimate the probabilities of the soil classes precisely. In fact, the real objective in such problems is to estimate the soil types at unknown locations correctly. In other words, the rank of the estimated probably of each soil category at each location as a representative of the category which is going to be assigned to each location, are more critical than the probability values themselves. For instance if the probability of the existence of a soil class, let say $k$, in an estimation position is the highest compared to those of the other soil classes, then the conclusion will be that the existing soil category at that point is the soil class $k$.

In this study, to perform this form of the cross-validation for the categorical variables, the estimated soil class (i.e. the most probable soil class) was compared to the true existing soil class in all sample locations. Then, the ratio of the correctly estimating the true soil class in sample locations was calculated for the model. Another criterion considered for the evaluation of the quality of the estimations was the average probabilities of the true classes (Deutsch 2002, pp. 304-308). Hence, this average will be high when the probabilities of the true class are also high:

$$
C=\left(\frac{1}{N}\right) \sum_{i=1}^{N}{ }_{i}\left(u_{i} ; k\right) \mid \text { true }=k
$$

In this equation (Deutsch 2002, p. 308), C denotes the closeness measure that quantifies how accurate the probabilities are for the true classes, $p\left(u_{i} ; k\right)$ is the probability of the true class achieved from the indicator kriging during the cross-validation process at the estimation location of $u_{i}$, and $k$ is the true class exists at that location.

An important point in the cross-validation of a three-dimensional well-bore data-set is that if the "leave-one-out" approach is applied in a sample-by-sample way, the goodness of the estimations will normally be evaluated as being quite higher because the samples are much denser in the vertical direction and the estimation will be affected most by the samples with very near distances to the estimation point. Therefore, the probability of correctly estimating the classes will be evaluated as noticeably high as well. To solve the problem of overrating the rightfulness of the soil categories estimations, an alternative method is to use the cross-validation method well-by-well instead of point-by-point. In other words, in every estimation step during the cross-validation, all the points of a well is omitted and estimated using the neighboring points from other boreholes and so forth for 
the other wells to the end of procedure. Table 3.1 summarizes the cross-validation of indicator variograms and estimation models. These results were applied to evaluate the goodness of estimation models which were employed in the indicator kriging of the soil categories in present study.

Because the soil categories were represented by the consequent numbers (i.e. 1, 2, 3, 4) and in the case of being unsure between two consequent categories in samples, by the index of the lower category plus 0.5 , i.e. 1.5 and 2.5 , the estimated soil categories in these points also entail uncertainty. For instance, if the estimated soil category is 2 and the sample index is 1.5 , the estimation is probably either correct or wrong. If a sample actually belongs to the category 1 , the estimation is wrong. However, if the real category of the sample is 2 , the estimation is correct.

There could be three decisions in such cases; accepting all the estimation cases where the absolute difference between the sample category index and estimation is 0.5 or 0 as being correctly estimated (optimistic view), rejecting all the cases with the difference of 0.5 as well as any greater difference (pessimistic view), or accepting the half of the cases with the soil index difference of 0.5 and rejecting the half of them. In addition, because the decision about the estimated category that occurs in a point is based on the selection of the category with the highest estimated probability, it was suggested that, the mean of the highest probability (among the four estimated probabilities for the four categories) at cross-validation points also could be a representative of the quality of the estimations. This is because, the higher the probability of a selected category, the more reliable the decision of the selecting this category would be. In other words, if the probability of the selected category which is assigned to this point is more clearly higher than those of the other categories, the indicator kriging will suggest more strongly the presence of that category in the mentioned location. Therefore, another criterion could be suggested to evaluate the estimation qualities for categorical variables using cross-validation technique.

\section{(b) Jackknifing:}

Another scheme for validating the variogram and estimation models is to use the "keepsome-back" jackknifing technique in which the available data-set is split into two subsets. One of these subsets of the original data-set can be re-estimated from the other nonoverlapping data-sets (Deutsch 2002, pp. 115-125; and Deutsch and Journel 1998, p. 94). 
Then, the true and estimated values can be compared to assess the quality of the estimation models. Suppose that the analyzer puts the $50 \%$ of the available samples aside and transfers them to another data-file. Therefore, one of the separated subsets from the original data-set is assumed as unknown and the other subset as known. Then its values are estimated using the "known" samples. Subsequently, the real and estimated values can be compared together similarly to the methods described before for the cross-validation.

To perform a Jackknife analysis for the indicator kriging models, the boreholes were divided into two sub-groups; wells with evenly-numbered borehole-indexes, and wells with oddly-numbered indexes. These indexes were given according to the $\mathrm{x}$-ascending values for the locations of these boreholes.

Then, one of the subsets of the original data was considered as known to estimate the other set which was regarded as unknown, and once with reverse assumption about which one as known and another as unknown. To assess the accuracy of the models, similar to the cross-validation studies, two measures were considered in this study; the percentages of correctly estimating the prediction points (i.e. the percentage of points where the estimated soil classes were equal to the true known classes), and the average probability of the true class. The outcome was more or less similar to that of the well-by-well crossvalidation of the indicator kriging models. The mentioned percentages (probabilities) have been summarized in Table 3.2.

Table 3.1 Summary of the cross-validation of the indicator variograms, point-by-point and well-bywell indicator kriging (IK).

\begin{tabular}{|c|c|c|c|c|}
\hline Measure & $\begin{array}{c}\text { Arithmetic mean } \\
\text { of the highest } \\
\text { probabilities in } \\
\text { each cross- } \\
\text { validation point }\end{array}$ & $\begin{array}{c}\text { Probability of } \\
\text { correctly } \\
\text { estimating the } \\
\text { existing } \\
\text { categories } \\
\text { without accepting } \\
\text { o.5 difference in } \\
\text { category codes }\end{array}$ & $\begin{array}{c}\text { Probability of } \\
\text { correctly } \\
\text { estimating the } \\
\text { existing } \\
\text { categories with } \\
\text { accepting 0.5 } \\
\text { difference in } \\
\text { category codes }\end{array}$ & $\begin{array}{c}\text { probability of the } \\
\text { true class in } \\
\text { cross-validation } \\
\text { points }\end{array}$ \\
\hline $\begin{array}{c}\text { IK done point-by -point } \\
\text { cross-validation }\end{array}$ & 0.78069 & 0.70972 & 0.80213 & 0.65754 \\
\hline $\begin{array}{c}\text { IK done well-by-well } \\
\text { cross-validation }\end{array}$ & 0.56771 & 0.51483 & 0.60261 & 0.42461 \\
\hline
\end{tabular}


It can be inferable from Table 3.2 that the accuracy of the employed models has been relatively good and similar to the accuracy measure which has been inferred from the Jackknife method. The probability of correctly estimating a class, that seems a better criterion for estimating the accuracy in the estimation of the categorical attributes (because the final aim was to predict the soil class which exited in each position), shows higher values.

Actually, the probability of correctly estimating (inferred from well-by-well crossvalidation) in this case demonstrates that, well under 60-percent of the points have been estimated correctly.

Table 3.2 Summary of the jackknifing of the estimation models.

\begin{tabular}{|c|c|c|}
\hline Methods & $\begin{array}{c}\text { Jackknifing of data-subset 1 } \\
\text { by data-subset 2 }\end{array}$ & $\begin{array}{c}\text { Jackknifing of data-subset 2 by } \\
\text { data-subset 1 }\end{array}$ \\
\hline $\begin{array}{c}\text { Probability (or the } \\
\text { proportion) of correctly } \\
\text { estimating the true class }\end{array}$ & 0.57751 & 0.54804 \\
\hline $\begin{array}{c}\text { True class probabilities } \\
\text { average }\end{array}$ & 0.45685 & 0.44857 \\
\hline
\end{tabular}

The expectation of the author is that, the real accuracy of the estimations should be higher than the values reflected in Table 3.2. The reason is that, in the real estimation case on or near a borehole, the other samples along this borehole are also used in estimation and it is not necessary to omit a borehole totally during the estimation procedure.

\subsection{The indicator kriging (IK) analyses for the Göttingen test site}

\subsubsection{The general procedure}

To perform the IK analyses, as mentioned before, after assigning the indicator values to sample points, performing the grid-transformation, calculating the sample indicator 
variograms and modeling them, the variogram models were checked and improved. As the next step, the indicator kriging was performed and the probability of the occurrence of each soil class at each estimation point was estimated. At each estimation point, the soil classes with the maximum estimated probability were assigned as the estimated soil class which exists at that model location. Once the global statistics/geostatistics of the produced model were evaluated and the grid space was back-transformed into the original one, the three-dimensional models and sections were created and illustrated in the gocad software. To evaluate the performance of indicator kriging, two methods were tried in this study:

1. Using the kt3d program of the GSLIB software: the indicator values of each category were kriged separately, after achieving all the estimations (as the raw probability values), the indicator kriging estimations were normalized to sum-up to one, and the order-relation violations were corrected. Finally, the most probable soil class was drawn in each point, considering the estimated probabilities of the occurrence of each soil class from indicator kriging, and assigned to the corresponding location.

2. Using the ik3d program of the GSLIB software; the kriging was performed. In this program, the normalizations and order-relation problems corrections are done internally and automatically (embedded in the program), after estimating all the probabilities of the soil categories at once. Then using a separate computer program written by the author (a simple MATLAB $\operatorname{code}^{1}$ ) the most probable soil class was assigned to each estimation point.

The potential advantage of using the first procedure could be:

(1) The estimation variances of the indicators of each soil category would be available to have an evaluation of the uncertainties.

(2) The search parameters could be adjusted separately for each category whereas in $\mathrm{ik} 3 \mathrm{~d}$ all these parameters should be the same for all the categories.

However, for the practical ease, the second procedure has been preferred for final analyses and report here.

\footnotetext{
${ }^{1}$ This simple MATLAB code has been created by the author of this dissertation, Enayatollah Ranjineh Khojasteh.
} 
To check the accuracy and the quality of the estimation models (i.e. indicator variogram models and estimation parameters such as search radius and the number of points considered in estimations) and improve them, the cross-validation and Jackknife analyses were performed.

Finally, the back-transformation of the coordinates to the original coordinate system was performed. The three-dimensional models and sections were created and represented using gocad $^{l}$ software.

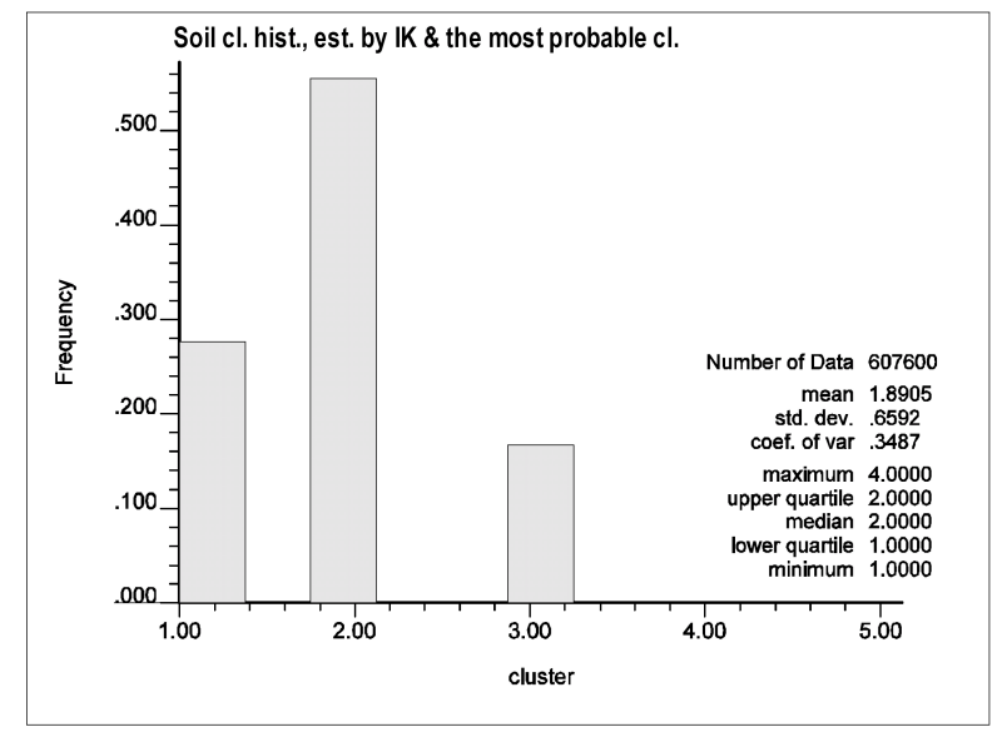

Figure 3.3 Histogram of the indicator kriging (IK) results for the soil class estimations in terms of the proportions of each soil category in estimated model. The class which held the highest probability was assigned to the estimation grid of the model in each estimation point.

As it was expected, the global statistics/geostatistics (i.e. the histogram of soil categories as well as their indicator variograms) of the estimated soil classes by IK method have not been reproduced the expected statistics/geostatistics adequately (see Figure 3.3, Figure 3.4, and Table 3.3 ).

It should be considered that, the output for indicator kriging is a set of probabilities for each category in every estimation point while the final goal is to predict the type of existing soil classes at each location. Therefore, the soil classes with the highest probability at each model point were assigned to the estimation points.

\footnotetext{
${ }^{1}$ Paradigm GOCAD 2009.2 (64-bits).
} 
Due to the huge number of the IK output file lines, after assigning the most probable soil class at each estimation location, achieving the frequencies and proportions of the estimated soil classes were noticeably difficult.

As a solution, the Textpad program and its sort function (to sort the file lines according to the soil class column) were applied, the lines of IK file for each soil class were separated in different Textpad pages, and finally the numbers of lines including these soil classes were extracted and written in a table (Table 3.3).

Table 3.3 Frequencies, proportions, and percentages of the estimated soil classes by the IK method and assigning the most probable class to each estimation grid of the IK estimation model.

\begin{tabular}{|c|c|c|c|c|}
\hline Soil class & $\begin{array}{c}\text { Soil classes } \\
\text { Frequencies } \\
\text { in the IK } \\
\text { model }\end{array}$ & $\begin{array}{c}\text { Soil classes } \\
\text { proportions in } \\
\text { the IK model } \\
\text { (\%) }\end{array}$ & $\begin{array}{c}\text { Expected } \\
\text { proportions } \\
(\%)\end{array}$ & $\begin{array}{c}\text { Deviation } \\
\text { rates (\%) }\end{array}$ \\
\hline cl1 & 168172 & $27.68 \%$ & $37.20 \%$ & $25.60 \%$ \\
\hline cl2 & 337906 & $55.61 \%$ & $42.30 \%$ & $31.47 \%$ \\
\hline cl3 & 101427 & $16.69 \%$ & $20.10 \%$ & $16.95 \%$ \\
\hline cl4 & 95 & $0.02 \%$ & $0.40 \%$ & $96.09 \%$ \\
\hline Sum & 607600 & $100 \%$ & $100 \%$ & $170.11 \%$ \\
\hline Average & 151900 & $25 \%$ & $25 \%$ & $42.53 \%$ \\
\hline
\end{tabular}

Figure 3.3 shows the histogram of the estimated soil clusters after assigning the most probable class to each estimation point specified by the indicator kriging method. It is clear from the histogram that the distribution of the estimated classes does not conform to that of the input data (see Figure 3.3 and Figure 2.8); the class 2 has been overestimated whereas the other classes have been underestimated. For analytical histogramreproduction checking of the IK estimated model, the deviation rates were considered. The term deviation rate has been explained more in part (2)a, section 5.3.3 from Chapter 5. The deviations of the proportions from the expected values were at minimum about $17 \%$ and in average almost $43 \%$ which are considerable deviations. Because of inadequate number of samples for the class 4 , the high deviation of its proportion from the expected value can be taken less seriously because even its estimated expected frequency should also be less reliable. For the two main classes; class 2, and class 1, the 
deviation rates were still considerable and above $25 \%$. In other words, the frequency of the biggest soil class (class 2) has been overestimated while those of the other classes have been underestimated. This feature is not similar to what is usually observed about the IK method for continuous attributes in which usually the frequency of the biggest class is reduced and the frequencies of the smaller classes are increased.

In Figure 3.4, the indicator variograms of the estimated model for soil classes generated by IK method and assigning the most probable soil class at each estimation grid-node have been illustrated. It is again clear from this figure that the variograms-reproduction for this estimation model was not so good (for example considering the range, nugget effect, and in some way sill) though the vertical variograms were considerably better except about the soil class 4 . For soil class 3, the variograms (and similarly corresponding soil-class proportions) were closer to those of the model. The variogram sills and ranges in the horizontal variograms were not in addition so different from those of the model while the nugget effects were very different in comparison to those of the model variograms. Since the soil class 4 had very few observations in the available samples, the expected or estimated statistics/geostatistics of it also should not be so reliable and the greater differences of them with those of the model can be due to such unreliability in these statistics.

In general, the sufficient reproduction of the global statistics is not expected in the IK estimation methods while the IK methods attempt to make a locally accurate estimation rather than globally accurate one. Therefore, the observed deviations in the global statistics are not so strange. However, as the Figure 3.6 to Figure 3.10, Figure 4.6 to Figure 4.14, and Figure 5.20 to Figure 5.36 represent, the general occurrence trends of the soil facies are similar to those of the SISIM and TSIM while the patterns produced by IK are much less scattered, and in this sense, the patterns are much smoother. Comparing these results to those of the similar cases such as He et al. 2009 demonstrates similar characteristics in these estimations and their statistical and geostatistical characteristics. 


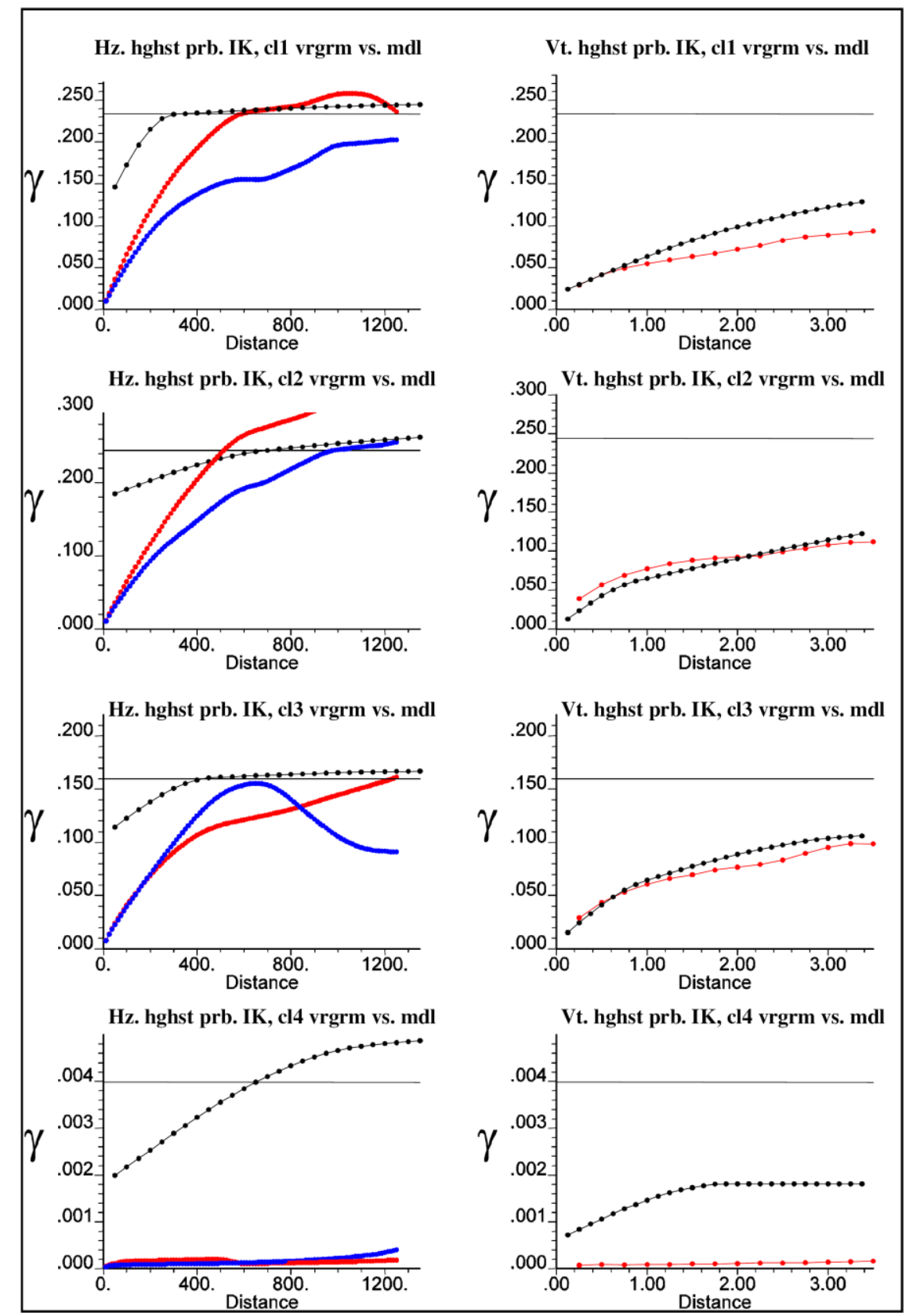

Figure 3.4 Indicator variograms of the indicator kriging (IK) results for the soil class estimations created by assigning the most probable soil classes to each estimation point. The graphs on the left and right side represent the horizontal and vertical indicator variograms, respectively. Black lines represent the model variograms while the red and blue lines demonstrate the indicator variograms of the estimated model. The red and blue lines on the left side show the indicator variograms of the horizontal estimation model along the NS and EW directions respectively. The red lines on the left graphs represent the vertical variograms of the estimated IK model.

\subsubsection{The effect of using a less number of samples on the estimations}

Using a less number of samples could affect the estimations in two ways by: 
(a) Reducing the clearness of the spatial variation structures of the sample data (i.e. the sample variograms).

(b) Reducing the numbers of samples contribute in the estimation stage. In this case, removing a group of the samples leads also to increasing of the samples spacing.

To check the effects of decreasing the number of employed samples on the clearness of the spatial variability structure and estimations goodness, four different subgroups of the whole data including; two-third, one-half (two subgroups), and one-third of the all boreholes were extracted. Though, only the results of $1 / 2$ samples have been brought here.
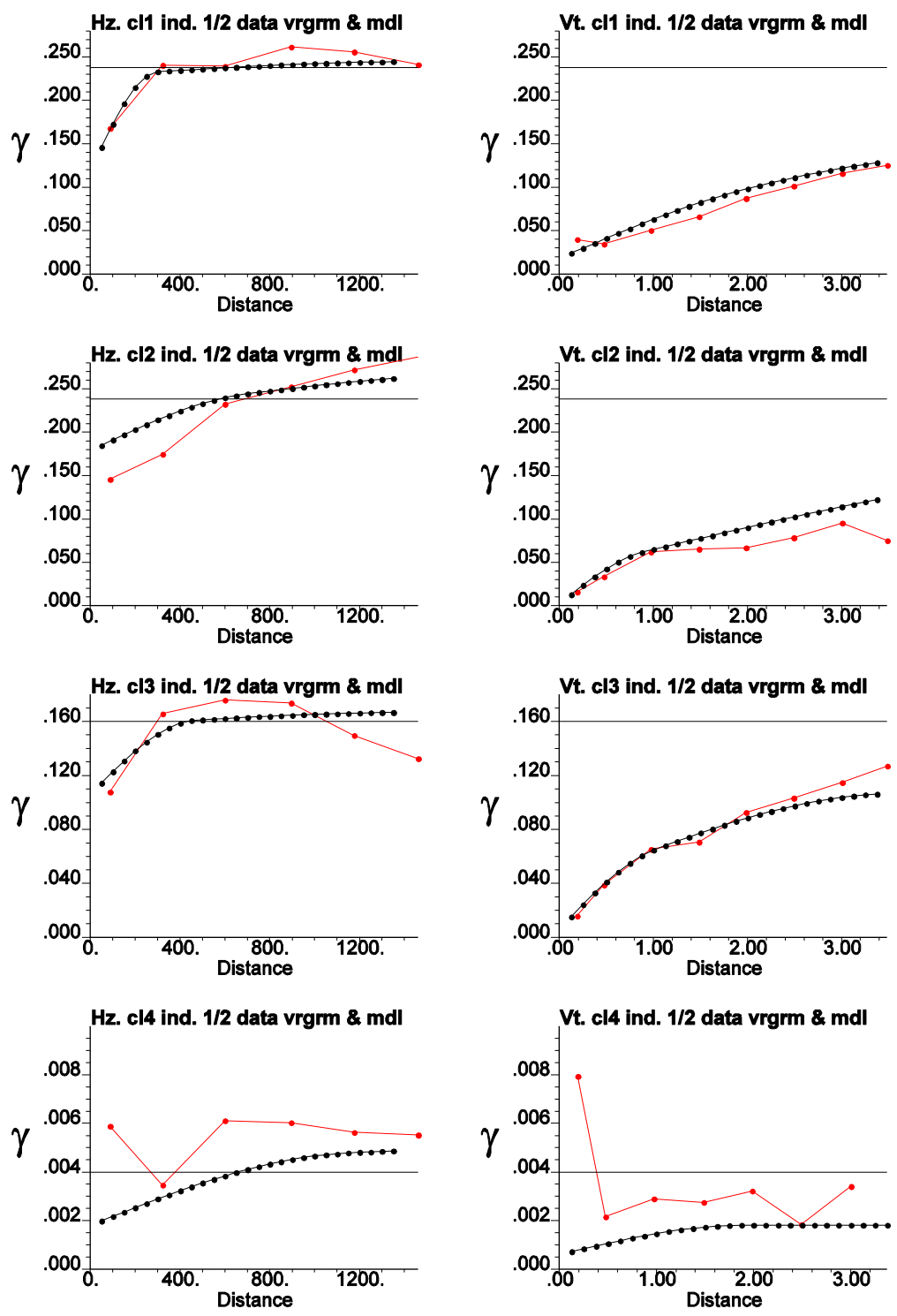

Figure 3.5 Sample and model indicator variograms of the soil categories in horizontal (left side graphs) and vertical directions (right side graphs). Sample variograms were calculated by the half of the existing boreholes. Red lines represent the sample variograms and the black lines represent the corresponding model. 
Then, theses data subgroups were examined once for their sample variograms clearness and once for estimation goodness using the jackknifing measures. These measures have already been explained in the previous section.

As stated above, the subgroup data-sets were used to calculate and plot new sample variograms.

Figure 3.5 represent the mentioned experimental indicator variograms of the subgroup data-sets as an example. It is clear that the general behavior of the indicator variograms have not been altered dramatically.

The effect of using a less number of samples on the quality of estimations was evaluated using the jackknifing and cross-validation methods which were explained in the previous section (see Table 3.1, Table 3.2, and Table 3.3).

In general, according to the, for the half of the boreholes (using the same variogram models as those of the complete data-set Table 3.3), the qualities of estimations, i.e. the percentage of estimating correctly, did not drop noticeably compared to that of the full data-set. Therefore, it can be suggested that a less number of samples even with about the half number of the existing boreholes in this study, can yield the estimation results with nearly similar estimation accuracy. The reason to suggest this, is that the rate of the correctly estimating soil class of the samples and the average of the true class probabilities from the well- by-well cross-validation are nearly the same as these rates in jackknifing in which the half of the boreholes were omitted in the estimation of the values of the other boreholes. In other words, when only one borehole was eliminated at each estimation stage (during the cross-validation procedure), the accuracy of the estimations was nearly the same as the time when the half of the boreholes were eliminated. The previous paragraph shows why considering the same variogram models for testing the effect of using fewer samples on the estimations can be a good approximation of the variogram models that could be employed in such cases.

The effect of using a less number of samples would be evaluated more reliably by comparison of two estimations/simulations generated by different number of input data point -by-point in which the less-dense data-set could be a selected part of initial data . Due to the huge volume of data-analyses tasks and the limited research time, this practice has not been made here. 


\subsubsection{Different search radiuses}

In the indicator kriging (IK) estimations, the considered search radiuses are among the most effective factors on the quality of the estimations. Decision for the most optimum search radius could be particularly essential. As a rule of thumb, one can multiply the variogram range by a coefficient greater than one (for example, $1.25,1.5,2$, etc.) to suggest a search radius, since it is better to consider a large-enough search radius that leads to a consistent estimation (Deutsch and Journel 1998, p.106). Using a larger search radius usually will increase the smoothing effect of the kriging whereas a smaller value will decrease this effect and highlight the local variations more (Sinclair and Garston 2002, pp. 236-237). On the other hand, in practice, a smaller search radius may cause limiting the estimable area. The computational time also, will obviously be longer when a bigger search radius is applied. Therefore, there should be a compromise between the larger and smaller search radiuses considering the modeling goals and expert's knowledge or opinion.

The effect of using different search radiuses on the quality of estimations was also evaluated here by means of the cross-validation method. The optimum search radius and other estimation parameters were selected to achieve the best cross-validation results by trial-and-error method.

\subsection{Models of the soil categories from the indicator kriging}

The three-dimensional model of the soil categories achieved by the indicator kriging according to the explained framework in Section 3.3.1 is brought here in several threedimensional (3D) sections. Figure 3.6 to Figure 3.10 illustrate these $3 \mathrm{D}$ sections. The models represent $2180 \mathrm{~m}$ distance in EW and $1580 \mathrm{~m}$ distance in NS direction with $15 \mathrm{x}$ exaggeration in the vertical direction. The results show clearly very smooth patterns and probably are not so realistic.

The interpretations and more discussions about this model have been brought in Section 5.3.6. 


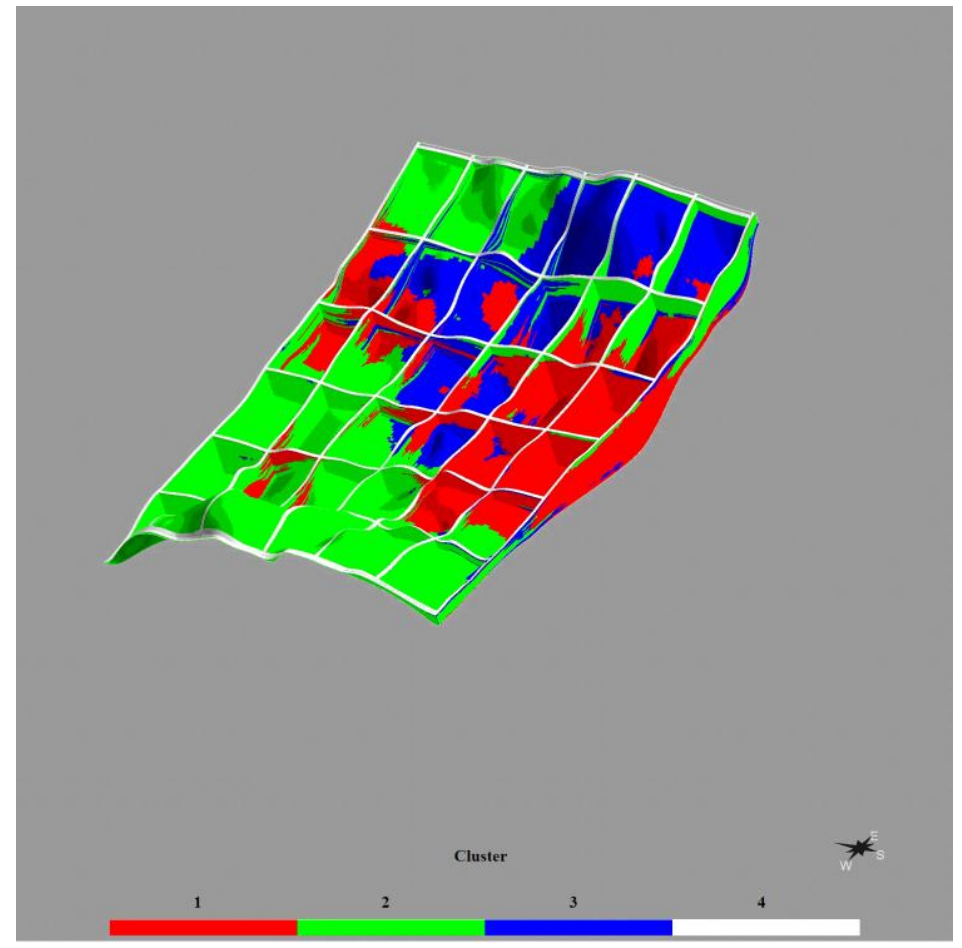

Figure 3.6 A perspective top view of the IK model including the lowermost surface (floor). The model represents a $2180 \mathrm{~m}$ distance in $\mathrm{EW}$ and a $1580 \mathrm{~m}$ distance in NS direction with $15 \mathrm{x}$ exaggeration in the vertical direction.

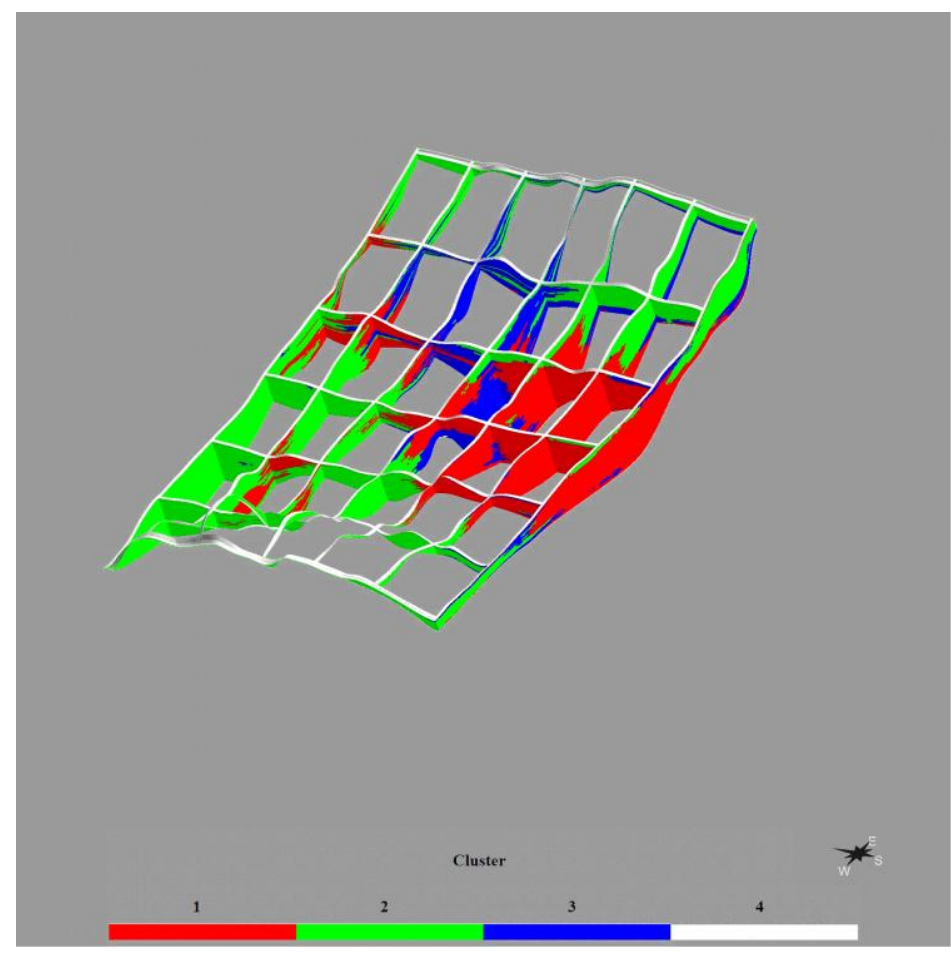

Figure 3.7 A perspective tilted top view of the IK model with fence diagram sections. The model represents a $2180 \mathrm{~m}$ distance in $\mathrm{EW}$ and a $1580 \mathrm{~m}$ distance in NS direction with $15 \mathrm{x}$ exaggeration in the vertical direction. 


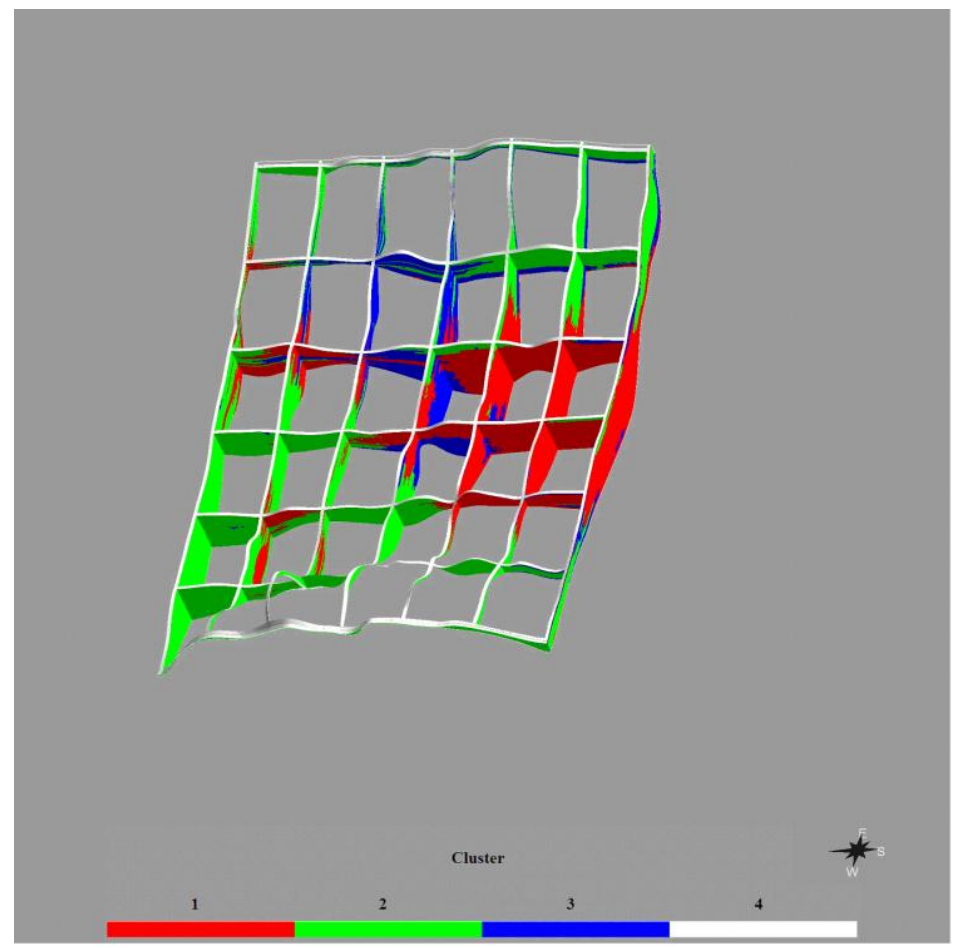

Figure 3.8 A perspective top view of the IK model with fence diagram sections along the basin. The model represents a $2180 \mathrm{~m}$ distance in EW and a $1580 \mathrm{~m}$ distance in NS direction with $15 \mathrm{x}$ exaggeration in the vertical direction.

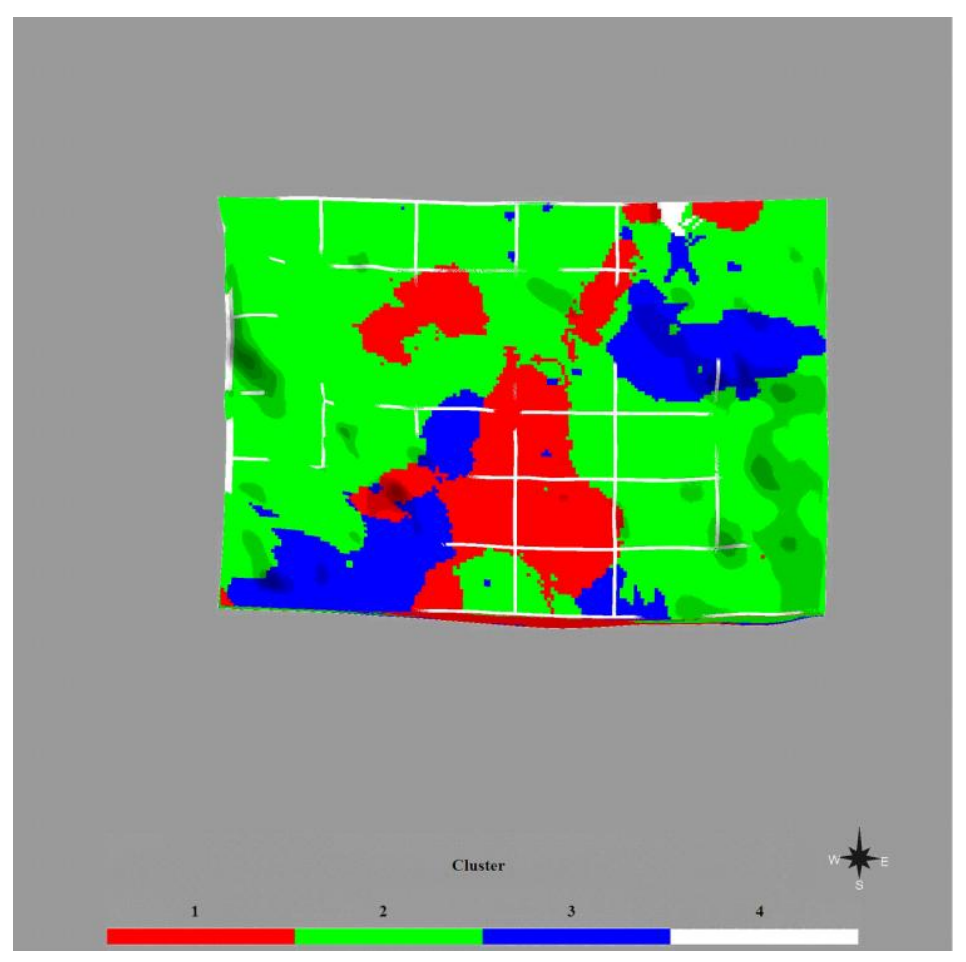

Figure 3.9 A perspective top-view of the IK model with fence diagram sections along the basin. The model represents a $2180 \mathrm{~m}$ distance in $\mathrm{EW}$ and a $1580 \mathrm{~m}$ distance in NS direction with $15 \mathrm{x}$ exaggeration in the vertical direction. 

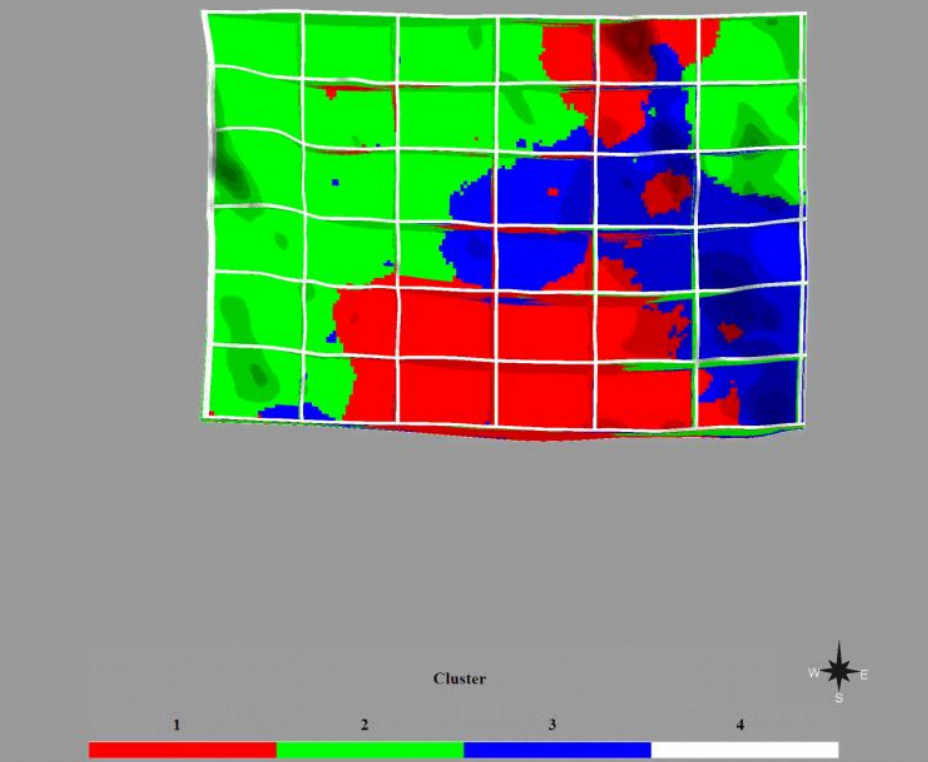

Figure 3.10 A top view of the IK model showing the lowermost surface (floor) of the basin model. The model represents a $2180 \mathrm{~m}$ distance in $\mathrm{EW}$ and a $1580 \mathrm{~m}$ distance in NS direction with $15 \mathrm{x}$ exaggeration in the vertical direction. 


\section{Sequential indicator simulation (SISIM) of the geotechnical soil classes in Göttingen test site}

\subsection{Overview}

As discussed before, the soil-types model (or similarly for facies types model) produced by indicator kriging is too smooth and unrealistic in geological point of view. To overcome this problem, the conditional geostatistical simulation was applied. The sequential indicator simulation (SISIM) was one of the geostatistical simulation methods employed in this study to obtain more sensible images of the subsurface (with more subtle and fine-scale changes). Then, the results out of different methods were compared. Geostatistical simulation or stochastic simulation is a series of techniques to produce a set of plausible scenarios for a known spatial structure that imitate the geological realities that are globally much more accurate (Caers 2005, pp. 11-13). The spatial uncertainty is a factor that can be evaluated in geostatistical simulation methods. For the facies modeling of diagenetically controlled systems, the SISIM method is broadly applied. The reason is that, the results of the SISIM method represent high variability, and at the same time, reflect the anisotropy and variograms corresponding to those of their underlying models (Deutsch 2002, p. 196).

The general procedure of a geostatistical simulation had been explained in Deutsch (2002, p. 196) as:

"Sequential indicator simulation consists of visiting all grid nodes of a simulation network in a random order."

A facies code is assigned at each simulation grid node during the SISIM method with the steps that can be summarized as following (Deutsch 2002, p. 196):

a. Find the neighboring data as well as the simulated grid nodes from the former steps.

b. Establish (estimate) the conditional distribution function by indicator kriging, that is, the calculation of the probability of the presence of each soil types at the current location, $\mathrm{p}_{\mathrm{k}}{ }_{\mathrm{k}}, \mathrm{k}=1, \ldots, \mathrm{K}$ 
c. Draw a simulated soil class from the mentioned conditional cumulative distribution function (ccdf).

All of the process explained above is repeated with different random number seeds to produce multiple realizations. A random path is considered to avoid producing artifacts. Such artifacts can stem from a regular path combined with a limited search. This restricted search is obviously required to limit the kriging matrix size (Deutsch 2002, p. 196).

Ideally the simulations should reproduce the initial data spatial structure and histogram. Clearly, the input conditioning data also should be honored.

\subsection{SISIM for the geotechnical soil classes of the Göttingen project}

For sequential indicator simulation of the geotechnical soil classes in Göttingen project, the sisim program of the GSLIB software was applied. The same variogram models that were used for indicator kriging (IK) were also employed in the simulations because these models have already been validated and admitted.

Similar to the IK results, some of the realizations have been depicted in threedimensional models and slices afterwards. The selection of the represented realizations was based on some geostatistical criterions, which guaranteed the reproduction of the expected statistics, as well as a number of geological considerations.

The number of 100 realizations was produced using the SISIM method. The simulation grid and search parameters were exactly like those of the IK model.

\subsection{Checking the realizations of the sequential indicator simulation (SISIM) method and selecting the best ones}

As discussed before, although the advantage of the kriging algorithms is accounting for the spatial variability structure, the smoothing-effect of them contradicts with the nature of the geological realities which are actually more variant and changeable. 
Nowadays, the geostatistical simulation techniques are getting more popular due to their capability to improve the heterogeneity characterization and joint uncertainty assessment (Leuangthong 2004, p. 131).

Geostatistical simulations create multiple likely scenarios called realizations. However, it is an important step to evaluate and check the produced realizations before they can be employed by practitioners in their real problems. In other words, some realizations which fit better to the reality of the statistical and geological heterogeneities should first be chosen.

Leuanthong et al. 2004 have discussed the minimum criterions for checking, accepting, and choosing the geostatistical realizations. These criterions include honoring the available data in their locations, the data statistical distribution, and the correlation structure.

\subsubsection{Criteria for checking the goodness of the simulation results}

\section{(1) Overview:}

To check the goodness of the produced simulations, four major criterions were considered in this study; the proportions- (histogram-) reproduction of the soil classes, the variograms-reproduction, transition-probabilities-reproduction, and the geological soundness of the simulation results. Clearly, the conditioning data also should be reproduced. It will be discussed later why the conditioning to the input data may not be met at times. The transition-probabilities measure was also assessed here as a complementary spatial variation index. Ideally, the simulations should meet all the mentioned conditions perfectly. In practice, some variations and fluctuations from the ideal case will be observed. The best realizations can be and were selected here in terms of the mentioned criteria.

Theses parameters can be evaluated either for single simulations or for all the simulations at once. The realizations were checked here for all realization one-by-one and the quality of them were assessed.

The input data and histogram reproductions have been explained very briefly in the coming section, because they have been elaborated in the next sections related to the evaluations of the transition-probability Markov chain simulations. 
Each criterion has been explained separately in the following sections.

\section{(2) Honoring the input data and histogram reproduction for the realizations of the SISIM method:}

As mentioned above, the input data should be honored in their locations. Although the indicator kriging, as the base of the SISIM method to estimate the local probability distribution functions, is an exact estimator and expectedly all the SISIM results should honor the input data but sometimes it does not so.

It has been explained in Section 5.3.2 why sometimes the input data is not wholly honored.

The details of the method for checking this criterion and its results also have been explained there (Section 5.3.2 ). The only result deserves mentioning at this point is that; in general, the input data was honored very well in all the SISIM realizations and even better than that of the TSIM (transition probability-based Markov chain simulation) method. Therefore, all the realizations from the SISIM method were considered acceptable in terms of honoring the input data.

In addition, the histogram- (i.e. proportions-) reproductions of the SISIM realizations were also checked for all the realizations. In other words, it was tested whether or not each realization has reproduced the expected proportions of each soil class in an acceptable statistical tolerance.

According to these criterions (see Sections 4.3 and 5.3) the realizations which reproduced the expected proportions sufficiently were suggested for evaluation in the next steps. The more detailed explanations and results about this test for the SISIM method also has been brought in the next sections, related to the transition-probability Markov chain method.

\section{(3) Variogram-reproduction for SISM:}

Figure 4.1, Figure 4.2, and Figure 4.3 represent the horizontal and vertical simulation variograms for realization number 37,61 , and all realizations produced by SISIM (sequential indicator simulation) method, together with their corresponding variogram models. The realization 37 was considered to be the best suggested realization among the all generated 100 realizations of the SISIM method in terms of the histogram- (i.e. proportions-) reproduction. Therefore, it was anticipated that the variograms of realization 
37 generated by SISIM can reproduce the expected model variogram sills (or even the whole model variogram) better. This feature is because the expected indicator variogram sill is a function of the proportion of a category:

$$
c_{i}=p_{i}\left(1-p_{i}\right)
$$

where $c_{i}$ is the expected sill for the indicator variogram of the category $\mathrm{i}$, and $p_{i}$ is the proportion of the category i.

In contrary, the realization number 61 with the greatest $\chi^{2}$ value among all the realizations of SISIM method was expected to demonstrate the weakest reproduction of the model variogram sills.

To evaluate the quality of variograms-reproduction using the existing graphs, four key parameters of the variograms were considered; nugget effect, sill, range, and variogram shape.

The evaluation was started with realization number 37 (Figure 4.1). It was observed that the nugget-effects which normally should reflect the short-scale variations, in the horizontal variograms were less than those of the model in most of the variograms, except for that of the class 4 , in which it is even higher. This means that, the simulation (number 37) has more continuous structure than expected except for the class 4 . About the class 4 , shorter horizontal structures have been distinguished in the geostatistical simulation too. Moreover, it might be speculated that the existing differences between the nugget effects of the models and the simulation horizontal variograms could also be due to the lack of the adequate information from the data samples in short. This is a usual case because the samples from the boreholes could not so densely be taken in the horizontal direction. In contrary, a dense sampling is available in the vertical direction and the short-scale structures can be reflected better in the vertical variograms. The variogram shapes were more or less similar to those of the model except that the simulation variograms had more fluctuations or sometimes a little more complex structures. The sills also were often very close to those of the models except for the variograms of the class 4 , especially in vertical direction. There are some differences in the variogram sills between the models and the simulation, especially for the horizontal variograms of the class 4 and somehow about class 1 . For the sills of the vertical variograms, the difference is clearer for class 2 and especially class 4 . For the vertical direction of the same realization, the nugget effects are quite similar to those of the variogram models. In general, the conclusion in the SISIM method could be that the variogram of the realization number 37 reproduced the model 
variograms reasonably well and the variogram reproduction can be accepted. Bearing in mind the Figure 4.2, it can be inferred that the variogram-reproduction of the realization 61 (with the weakest proportions-reproduction), in general was clearly much weaker compared to that of the realization 37. The red and dark blue lines in the left graphs show the horizontal variograms of the realization 61 in north-south, and east-west directions, respectively, whereas the purple and light blue lines represent the variograms of the similar directions for the simulation number 37 . The vertical variograms are plotted in the right-side graphs, in which red represent the variograms for the realization number 61 and purple for the realization number 37. It can be inferred from Figure 4.2 that, all of the horizontal variograms of the realization number 37 have reproduced the model variogram better than those of the realization number 61 . However, in the vertical direction, the variograms-reproduction has shown a different performance. The variogramsreproduction for the realization number 37 was just slightly weaker than that of the realization number 61 for the classes 1 and 2, nevertheless nearly the same, and still better than those of the realization number 61 for classes 3 and especially class 4 . As mentioned before, the general conclusion could be that the variograms-reproduction is much better for the realization number 37 than that of number 61 . This result could suggest that the variograms-reproduction of the realizations with a better proportions-reproduction can also be better, though this conclusion can not be generalized so strongly. In addition to the relation between the variograms-sill-reproduction and proportions-reproduction (Equation (4-1)), one could also tentatively refer to the relation between the slope near the origin of a variogram, the proportion and mean length of a category and on the other hand the probable relation between the proportion and the mean length of a category (Equation (515 )). If the slope near the origin of the variograms as well as the variogram sills can be reproduced acceptably, the variograms-reproduction could also become very near to fulfillment. In other words, such result and inference can suggest the proportionreproduction as a key criterion to find the best realizations which most probably would reproduce the spatial variability of the simulation models as well. Figure 4.3 represents the spectrum of the variograms of the simulations for the realizations number 1 to 100 as well the corresponding variogram models. The variograms cover a broad area in the graph. This phenomenon obviously refers to the high degree of variations (in terms of the spatial variability structure) among the different realizations. The degree of variations among the different realizations, for example in terms of the diversity of the simulation variograms of each soil class, also can be considered as a guide to evaluate the robustness 
and consistency of the simulation method. This is because, the simulation methods that produce more similar simulation variograms (the variograms of the simulation results), should obviously have closer similar simulation results in different realizations. Therefore, a less dispersions of the variogram plots can be interpreted as a less degree of uncertainty in the simulation method and vice versa. An interesting point is that; in spite of using isotropic variogram models for the simulations, in the horizontal direction the simulation variograms represent a clear anisotropy in the two horizontal directions of north-south (y-coordinate) and east-west (x-coordinate). The applied variogram models can be considered as the average variograms of the different directions that could not be detected from the original data due to the lack of sufficient samples in this direction. The directional variograms of the simulations in the north-east and east-west directions were represented by the red and blue or purple and light blue lines respectively.

The solid black lines always represent the model variograms in these graphs. 

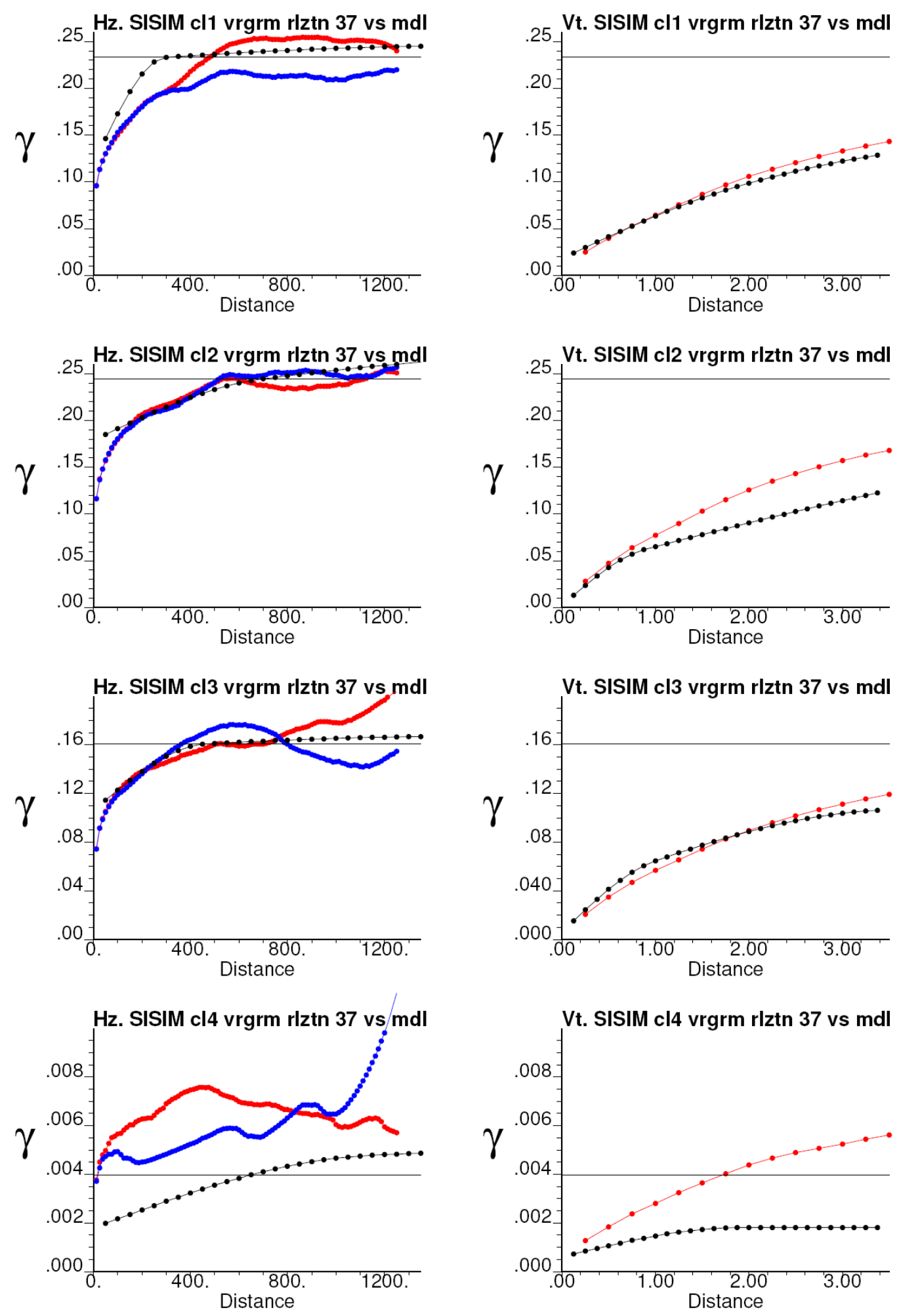

Figure 4.1 Checking the variogram-reproduction of realization 37 for SISIM method. Red and blue lines represent the simulation and black lines show the model variograms. In the left side graphs which represent the horizontal variograms, the red lines illustrate the variograms of the simulation in the NS, and the blue lines represent the simulation variogram in EW direction. The right-side graphs show the vertical variograms in which the red lines show the simulation variogram. 

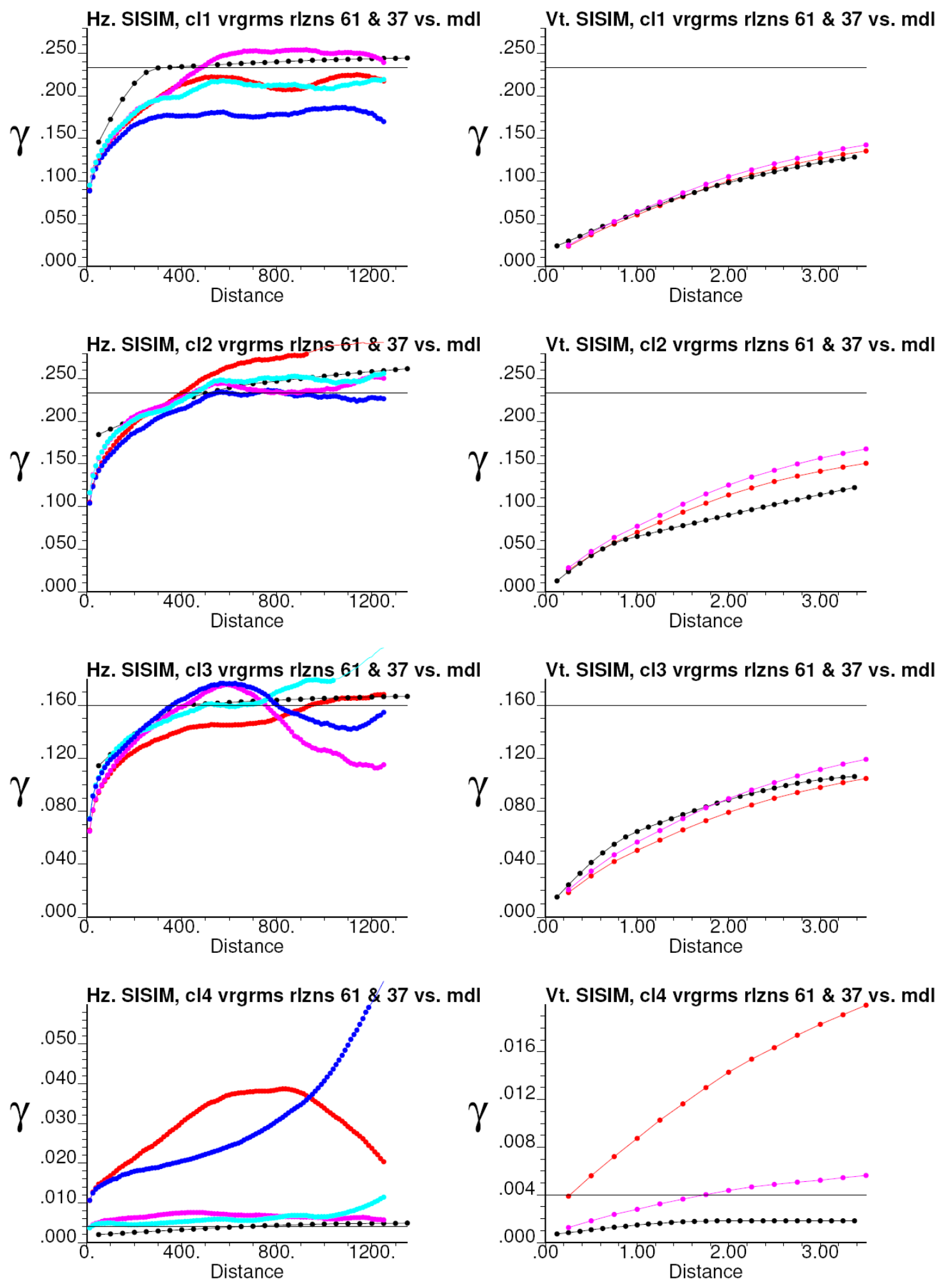

Figure 4.2 Checking the sequential indicator simulation (SISIM) variograms reproduction for realization numbers 37 and 61 . Colored lines represent the simulation and black lines show the model variograms. The left side graphs represent the horizontal variograms whereas the right side graphs are the vertical variograms. The red and purple lines in the horizontal variograms show the NS, and the dark and light blue lines in the horizontal variograms represent the EW directions, respectively. The purple and light blue lines represent the variograms of the realization 37 and the red and dark blue lines show the variograms of the realization 61 . Red and purple lines in the vertical variograms show the vertical sample variograms of the realizations 61 and 37 , respectively. 

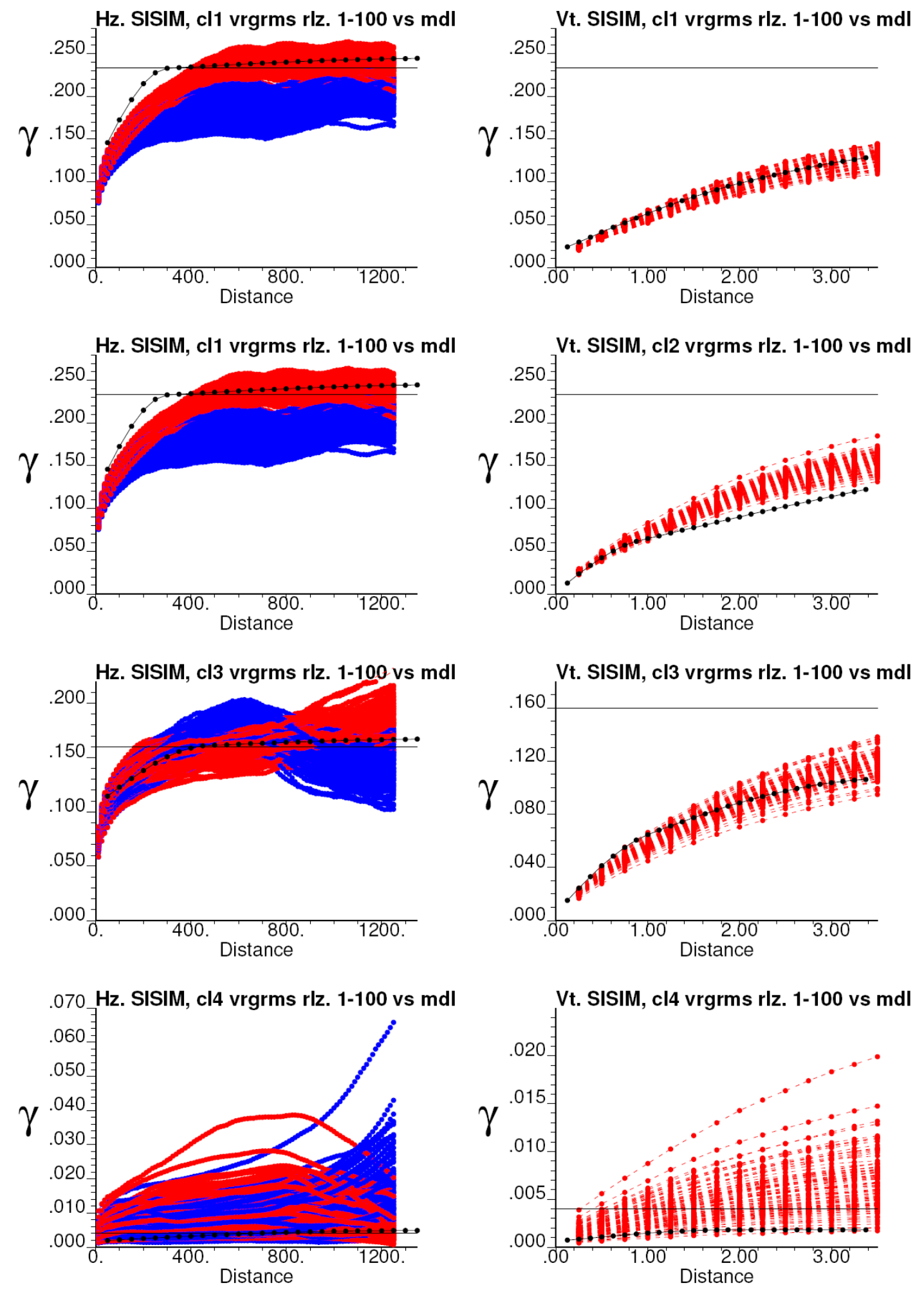

Figure 4.3 Sequential indicator simulation (SISIM) variograms for 100 realizations (red and blue lines) in horizontal (left graph; red for EW, and blue for NS) and vertical directions (right graphs) versus model variograms (black lines). 


\subsubsection{The transition-probabilities- reproduction of the SISIM realizations:}

The variograms do not have the capability of fully reflecting the interclass spatial variability dependence structures among pairs of the (soil) classes. Therefore, the quality of reproducing spatial variability structure could not be evaluated sufficiently for the simulation results by merely using their indicator variograms. Therefore, it was considered to plot and evaluate the transition-probabilities (TPs) among the pairs of the soil classes in principal directions as well to accomplish the spatial variabilityreproduction evaluations.

In this study the TPs of the realizations were compared to their corresponding Markov chain models. The models of TPs were taken from the TPs and Markov chain model analyses results which have been addressed in the next chapter.

As the extreme cases, the realizations with the best and worst proportions-reproductions, i.e. realizations 37 and 61, for the SISIM method were evaluated for their TPsreproduction. In other words, it was checked whether or not the TPs of these realizations correspond to their related Markov chain models?

Similarly, this test was conducted for the realizations produced by the transitionprobability Markov chain (TP/MC) simulation method as well.

Figure 4.4 and Figure 4.5 depict the TPs of the chosen realizations 37 and 61 generated by the SISIM method. It is clear from these figures that the TPs-reproduction of the realization 37, in average, was better than that of the realization 61. In general for the SISIM method, the TPs-reproduction of the realization 37 could be considered rather reasonable, especially when the emphasis is put on the starting lags of the TPs model. In the larger lags, the TPs-reproduction becomes weaker.

More details about the spatial variability structure-reproduction have been addressed in the next chapter. 


\section{Geotechnical classes Goettingen soils Hz. Transition Probabilities}
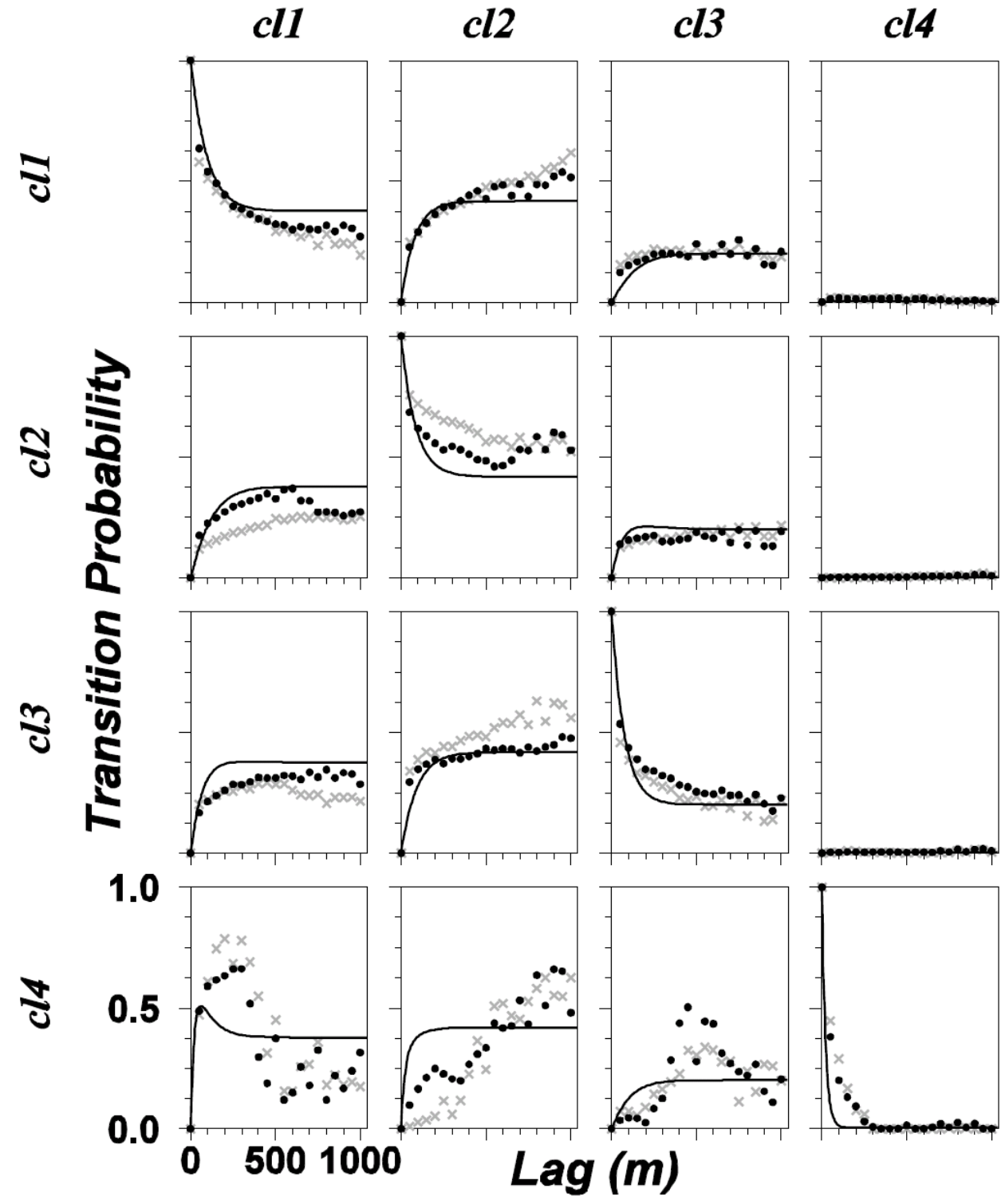

SISIM_OK_r37

SISIM_OK_r61

MC model

Figure 4.4 The transition-probabilities of the SISIM realizations number 37 (points) and 61 (crosses), versus their corresponding Markov chain models for the horizontal direction of the existing soil classes in the study zone. The calculations have been conducted in 


\section{Geotechnical classes Goettingen soils Vt. Transition Probabilities}
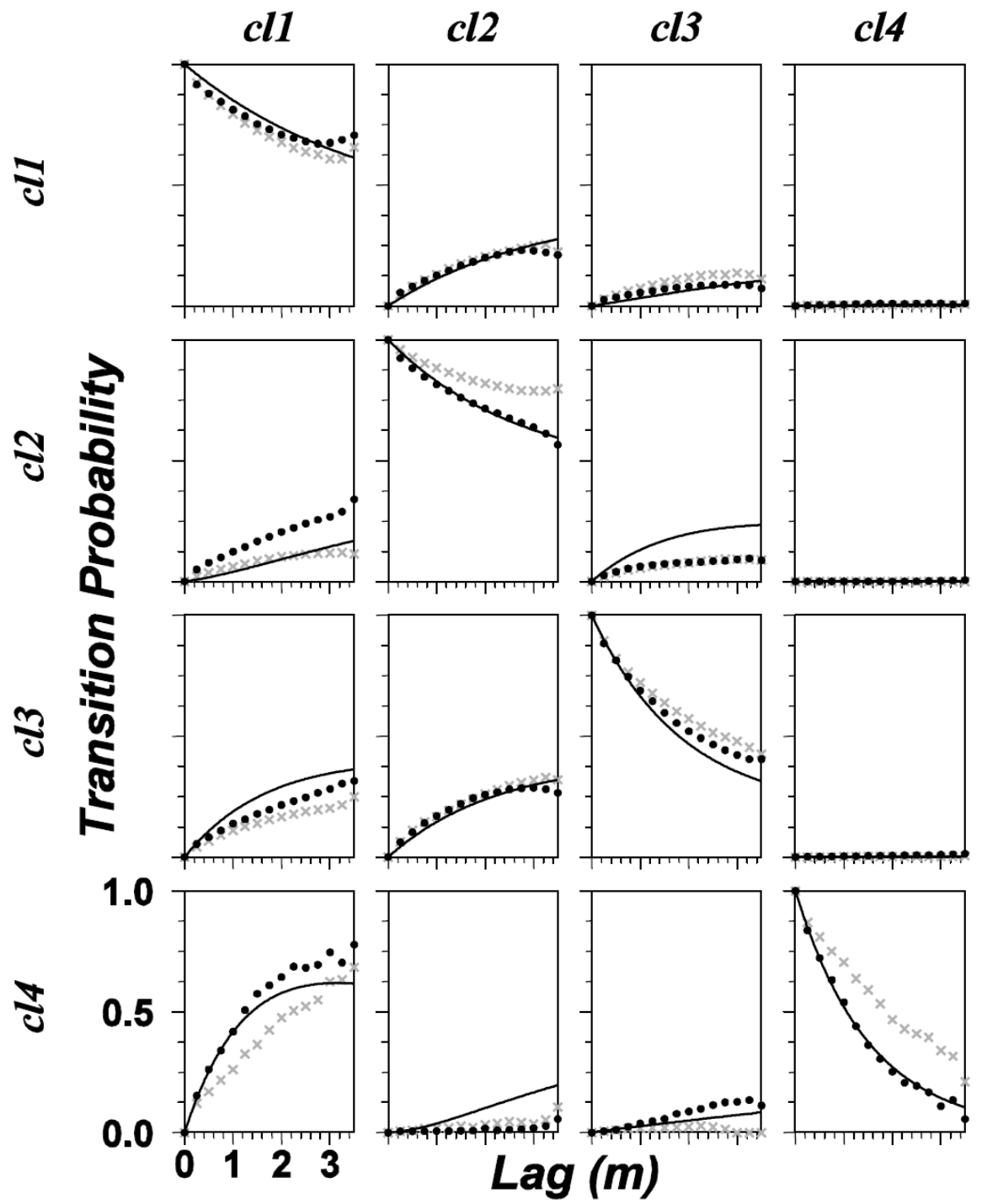

SISIM_OK_r37

SISIM_OK_r61

MC model

-....

Figure 4.5 The transition-probabilities of the SISIM realizations number 37 (points) and 61 (crosses), versus their corresponding Markov chain models for the horizontal direction of the existing soil classes.

\subsection{Three-dimensional sections of the selected SISIM realization:}


As mentioned above, the realization 37 which represented the best statistical fulfillment of the minimum criteria was selected as the best realization in terms of the mentioned criterions.

Moreover, the geological soundness of this realization also had to be evaluated. Hence, a set of sections were produced in the GOCAD software and were brought here. The discussions about the geological soundness of this realization, considering the generated sections have been brought in the next chapter to make it easier to compare the results from various methods.

Some of these sections have been represented in Figure 4.6 to Figure 4.14.

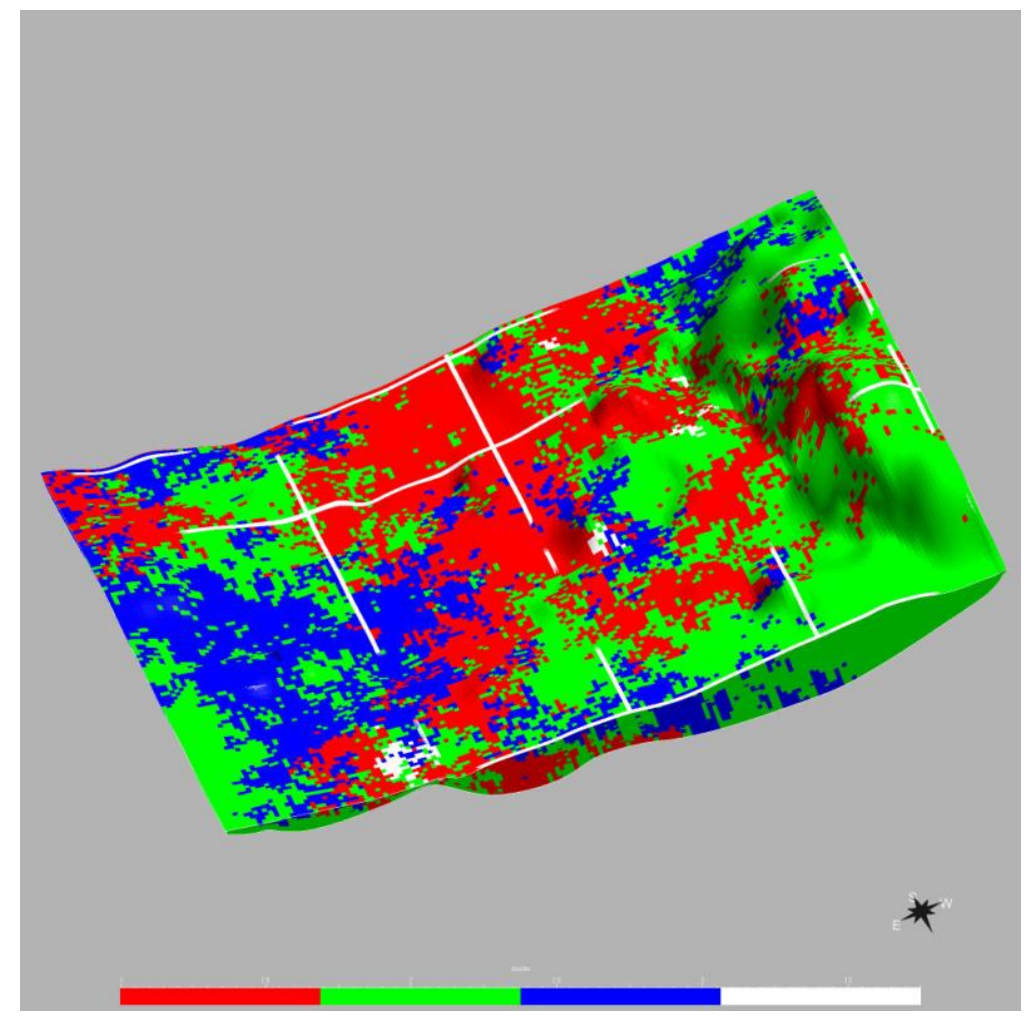

Figure 4.6 A perspective top view of the realization 37, generated by the SISIM simulation method. The model represents a $2180 \mathrm{~m}$ distance in $\mathrm{EW}$ and a $1580 \mathrm{~m}$ distance in NS direction with $15 \mathrm{x}$ exaggeration in the vertical direction. 


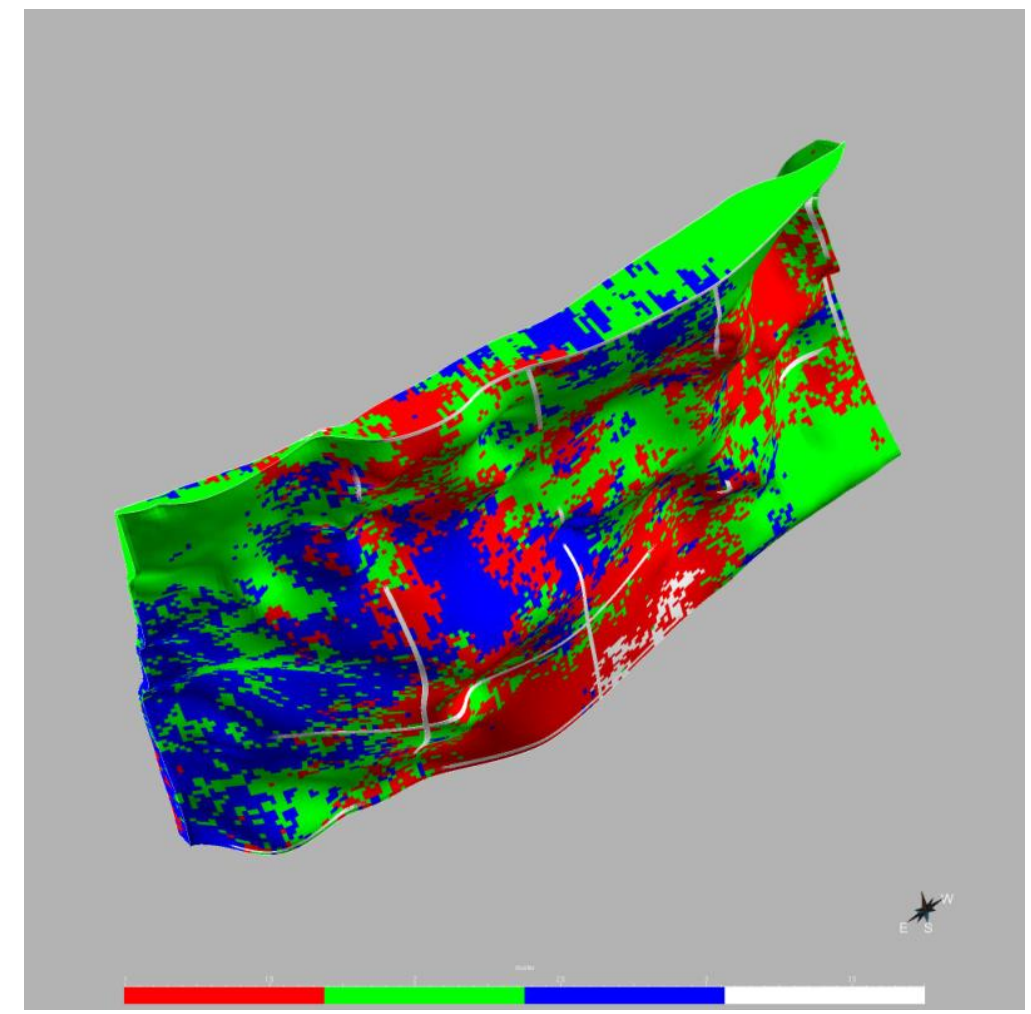

Figure 4.7 A perspective bottom view of the realization 37, generated by the SISIM simulation method. The model represents a $2180 \mathrm{~m}$ distance in $\mathrm{EW}$ and a $1580 \mathrm{~m}$ distance in NS direction with 15x exaggeration in the vertical direction.

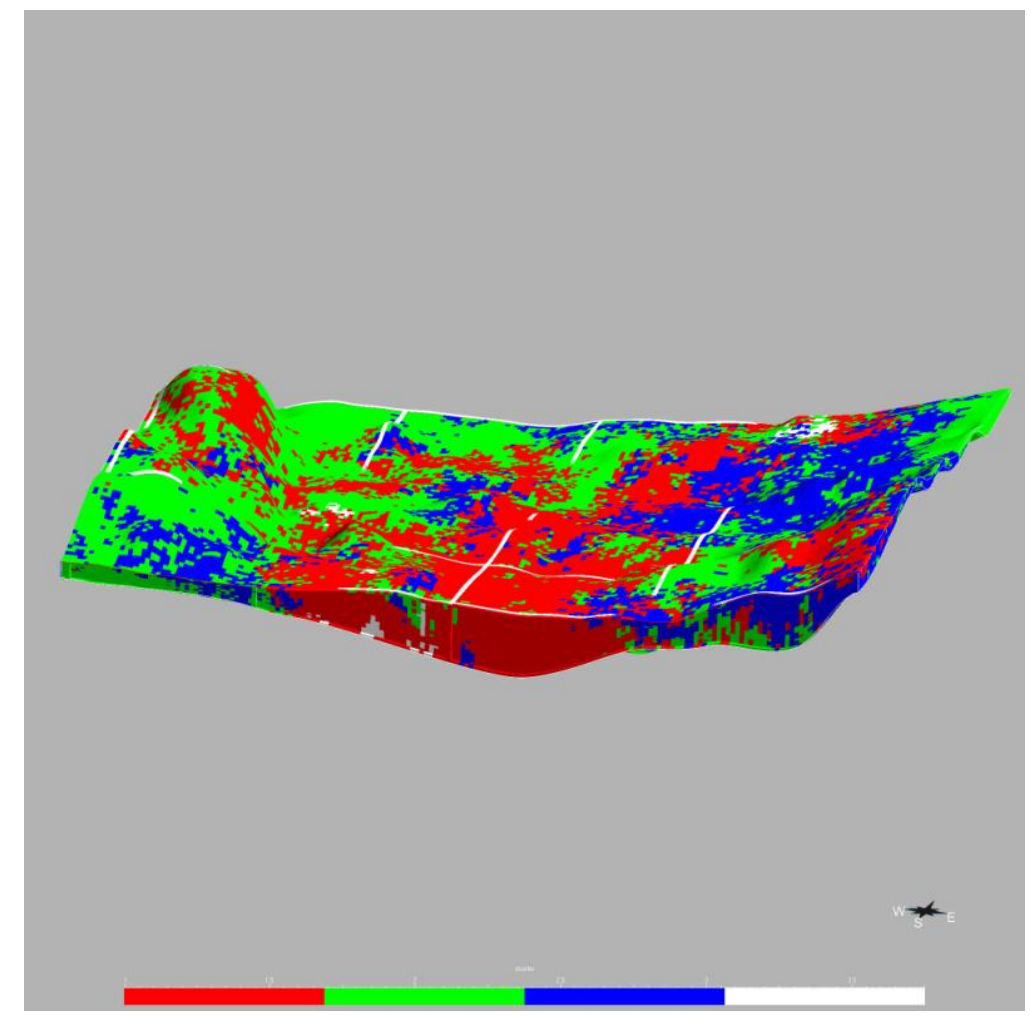

Figure 4.8 A perspective top side view of the realization 37, generated by the SISIM simulation method. The model represents a $2180 \mathrm{~m}$ distance in $\mathrm{EW}$ and a $1580 \mathrm{~m}$ distance in NS direction with $15 x$ exaggeration in the vertical direction. 


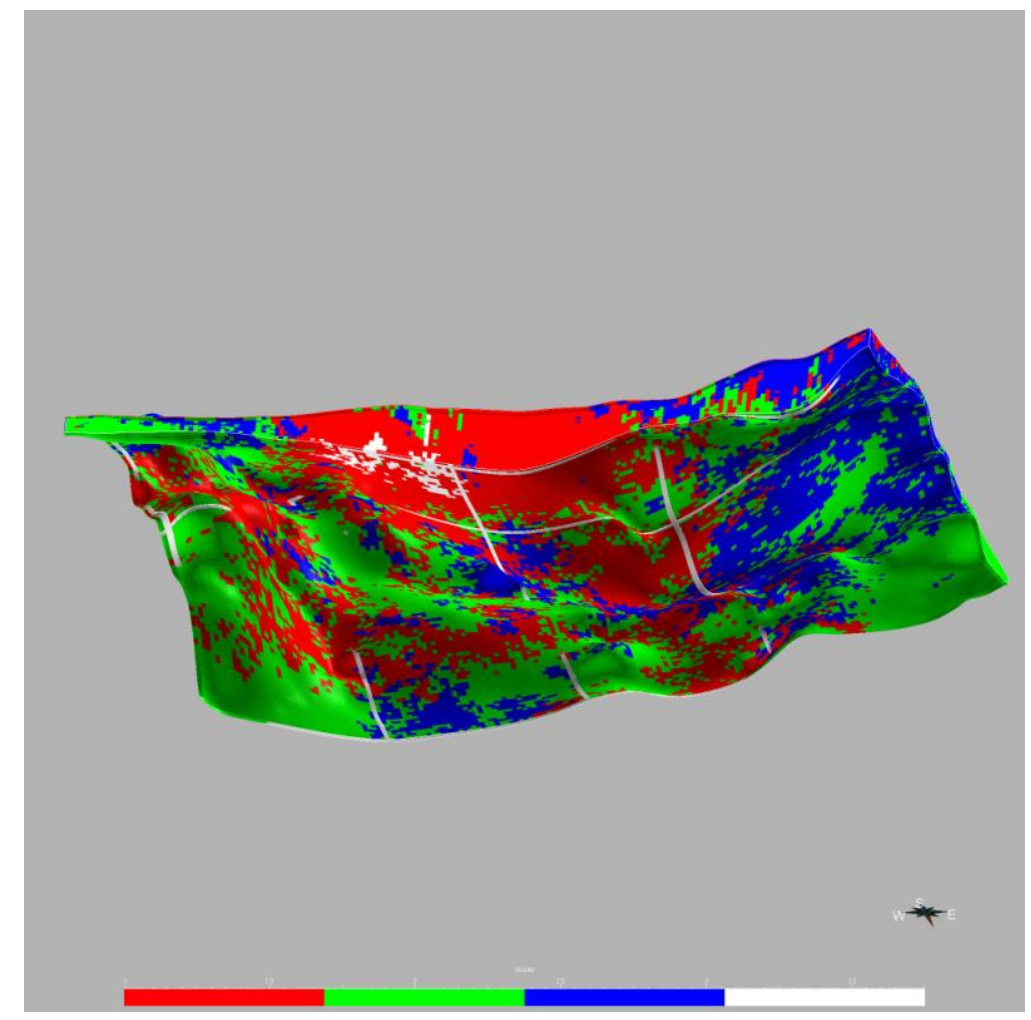

Figure 4.9 A perspective bottom side view of the realization 37, generated by the SISIM simulation method. The model represents a $2180 \mathrm{~m}$ distance in $\mathrm{EW}$ and a $1580 \mathrm{~m}$ distance in NS direction with $15 x$ exaggeration in the vertical direction.

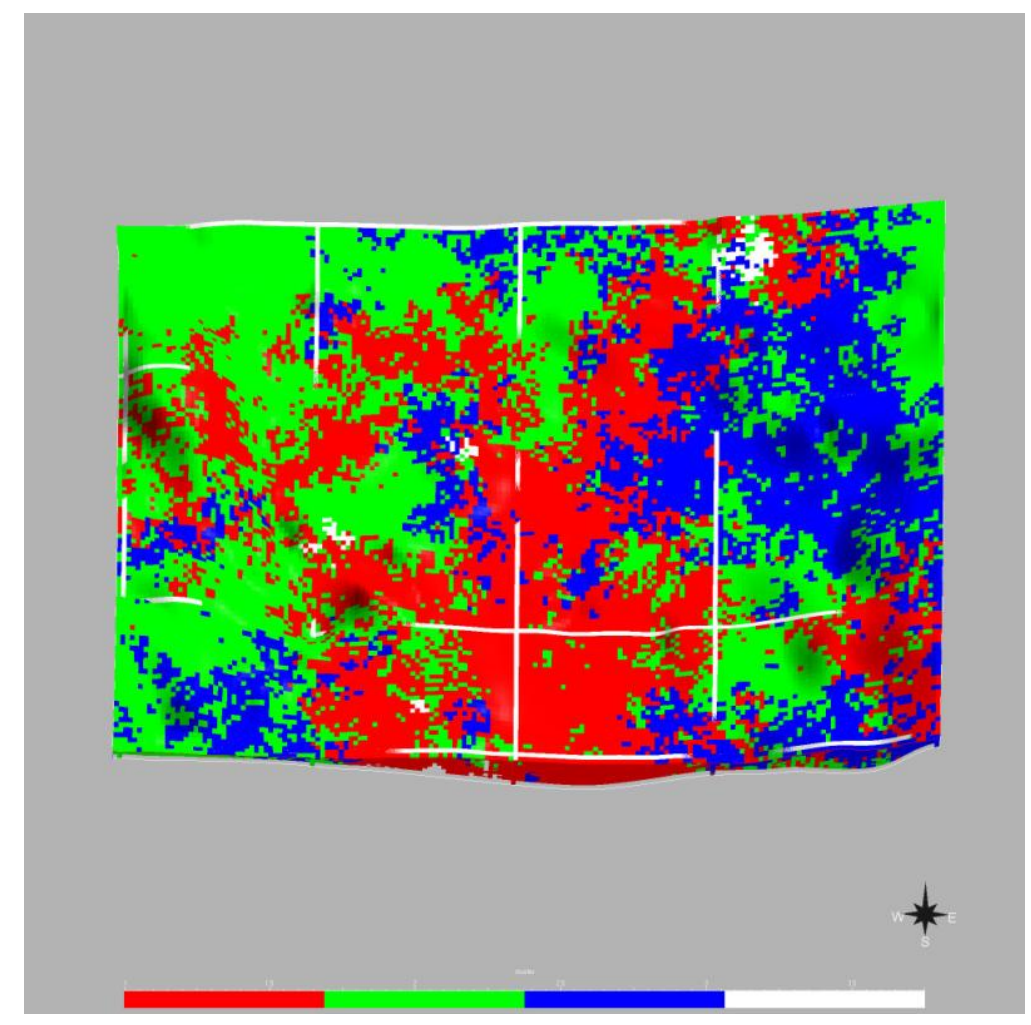

Figure 4.10 A top view of the realization 37, generated by the SISIM simulation method. The model represents a $2180 \mathrm{~m}$ distance in $\mathrm{EW}$ and a $1580 \mathrm{~m}$ distance in NS direction with 15x exaggeration in the vertical direction. 


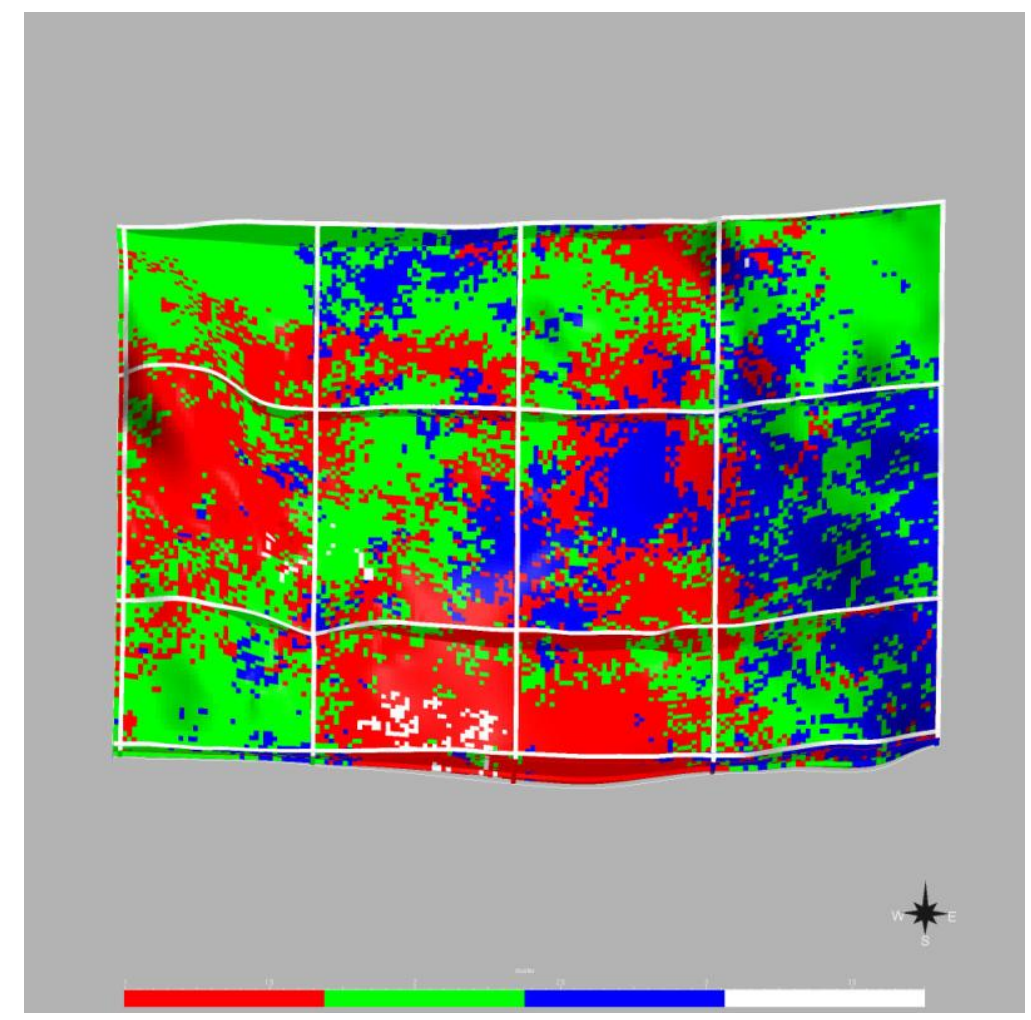

Figure 4.11 A top view of the realization 37, generated by the SISIM simulation method showing the lowermost surface (floor) of the basin. The model represents a $2180 \mathrm{~m}$ distance in $\mathrm{EW}$ and a $1580 \mathrm{~m}$ distance in NS direction with $15 x$ exaggeration in the vertical direction.

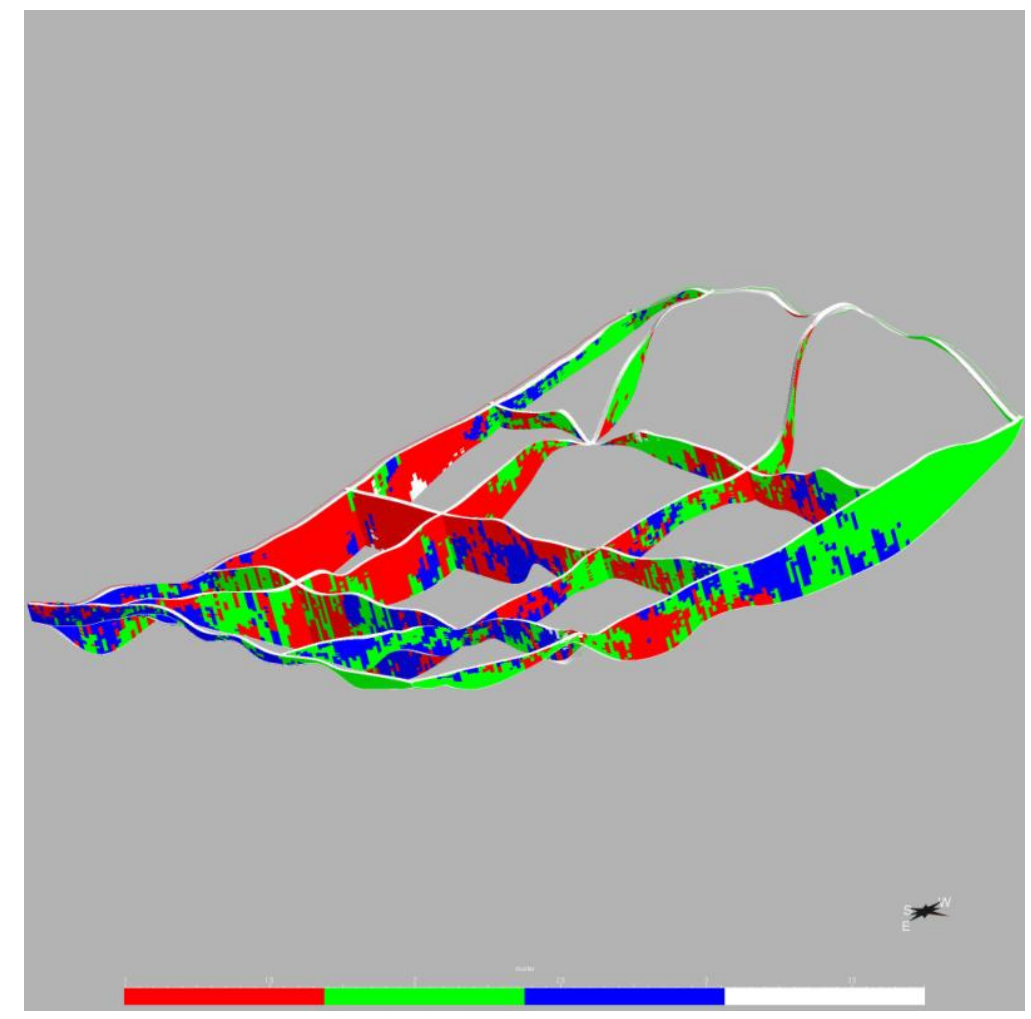

Figure 4.12 A perspective top side fence-section view of the realization 37, generated by the SISIM simulation method. The model represents a $2180 \mathrm{~m}$ distance in $\mathrm{EW}$ and a $1580 \mathrm{~m}$ distance in NS direction with $15 x$ exaggeration in the vertical direction. 


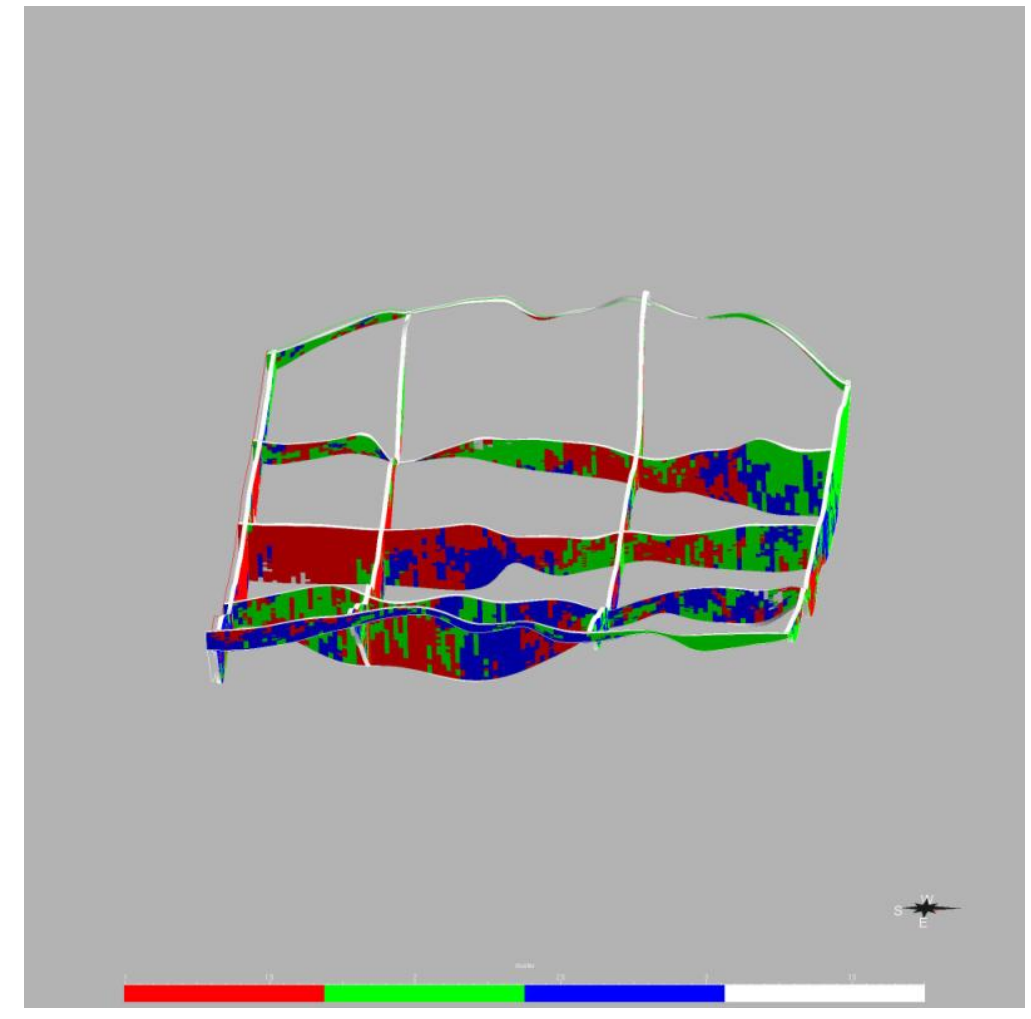

Figure 4.13 A perspective top side fence-section view of the realization 37 along the SN direction, generated by the SISIM simulation method. The model represents a $2180 \mathrm{~m}$ distance in $\mathrm{EW}$ and a $1580 \mathrm{~m}$ distance in $\mathrm{NS}$ direction with $15 \mathrm{x}$ exaggeration in the vertical direction.

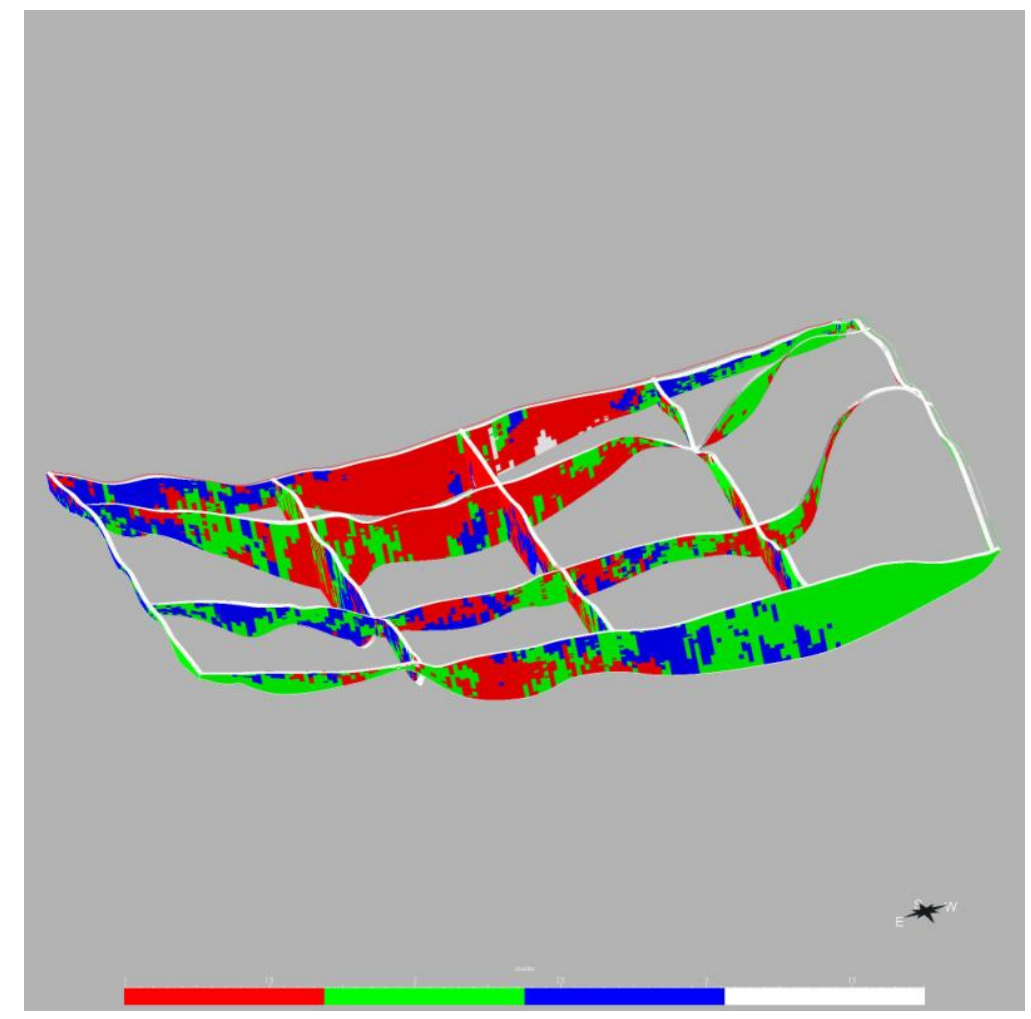

Figure 4.14 A perspective top side fence-section view of the realization 37 along the EW direction, generated by the SISIM simulation method. The model represents a $2180 \mathrm{~m}$ distance in EW and a $1580 \mathrm{~m}$ distance in NS direction with $15 x$ exaggeration in the vertical direction. 


\section{Transition-probability Markov chain (TP/MC) method for modeling subsurface heterogeneities in Göttingen pilot area, layer 5}

\subsection{Introduction}

The focus of Markov chain models of the transition-probabilities for geostatistical simulations is the probabilistic quantifying, evaluating, and modeling the changes from one state to another one in the time or space. Varieties of the states can be considered in this sense, such as having a rainy day, or a sunny day (in temporal view), or having a silt, clay, or sandstone along a traverse (in spatial view). Therefore, in the latter example, the existence of the clay in a point is one state (let say state 1), the existence of the sand is another state (let say state 2) and so on.

Markov chain (named after the Russian statistician, Andrei Markov) is a sequence of random variables, $X_{1}, X_{2}, X_{3} \ldots$ with the Markov property, i.e. the future depends solely on the present and not on the past. In other words, Markov chain is a sequence in which the state at one point depends partially, in a probabilistic sense, only on the previous state. Such a sequence with Markov property has an intermediate behavior between the totally random and absolutely deterministic sequence (Davis 2002, p. 172). In spatial Markov chains, the probability of going from one state (e.g. having clay in a point) to another state (e.g. having sand in the next point) depends solely on the nearest point(s). This Markov chain is stationary when the mentioned probability, is a function of a separation vector and not the location of the points (Carle 1999).

In the transition- probability Markov chain (TP/MC) method, the spatial variability structure is calculated and modeled using the transition- probabilities among various possible states instead of the variograms (Markovian view to the spatial variation structure). These transition- probabilities can be molded using a number of models for Markov chains. 


\begin{tabular}{|c|c|c|c|}
\hline \# $\mathrm{T}$ & $\mathbf{1}$ & $\mathbf{2}$ & $\mathbf{3}$ \\
\hline $\mathbf{1}$ & 15 & 5 & 1 \\
\hline $\mathbf{2}$ & 2 & 11 & 3 \\
\hline $\mathbf{3}$ & 3 & 0 & 9 \\
\hline
\end{tabular}

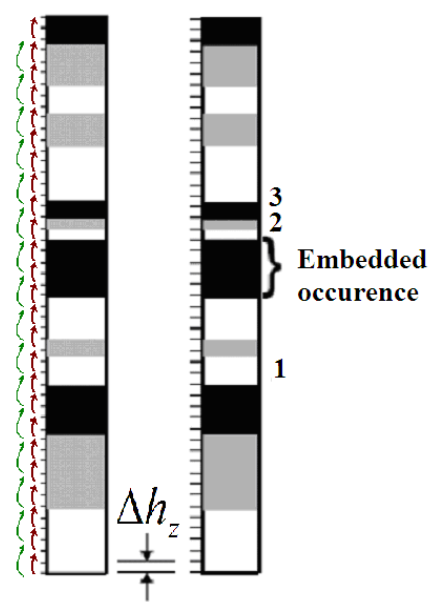

Figure 5.1 Transition-probabilities: $t_{j k}(h)=\operatorname{Pr}\{k$ occurs at $x+h \mid j$ occurs at $x\}$ as a function of separation vector and not the position in a stationary condition. The blue table summarizes the numbers of transition (\#T) from each facies type to another one in matrix format, for the example of the facies column in the logs seen at the left side of the figure, for a specific separation vector of $\Delta h_{z}$. The way of counting the transition for different lag-spacing (red and green arrows here) has been illustrated in the left-side log column (left graph are modified from Carle 1999, p. 8).

There are good reasons to adopt using the TP/MC approach in estimation stages, especially when the indicator variogram models demonstrate a considerably vague variability due to the insufficient sampling rates. In such cases, it would be necessary to inject more subjective or interpretive information into the model, for instance the mean length of the geological bodies or the possible sequence or neighboring of the layers.

The advantages of using transition-probability Markov chain approach over the traditional indicator geostatistical method can be summarized as following:

(a) An easier and improved integration of the geological subjective information of the facies architecture in modeling spatial variability especially when indicator variograms give too vague results (e.g. in too sparse or insufficient data-sets).

(b) Producing more consistent patterns with the plausible geology in the outcomes.

(c) Taking all the class inter-relations into account easily. This is especially correct when it is compared to the alternative methods such as covariograms and cokriging that demand special requirements in spatial variability modeling, including some constraints.

(d) Reducing the order-relation violations, in statistical sense of the modeling.

(e) Taking asymmetries into account.

It can be proven that the indicator kriging framework can be reformulated using the transition-probabilities. It is also possible to prove that the stationary transition- 
probabilities (in terms of the separation vector) can be fully modeled by an exponential model and a transition rates matrix like this (Carle 1999):

$$
T\left(h_{\varphi}\right)=\exp \left(R_{\varphi} h_{\varphi}\right)
$$

in which $T\left(h_{\varphi}\right)$ is he transition-probability matrix of the separation vector $h_{\varphi}$ in direction $\varphi$ and $R_{\varphi}$ is the transition rate matrix in the same direction.

More details about modeling the transition-probabilities will be explained in the next sections.

Having the transition-probabilities modeled, one can perform simulations based on them. More comprehensive descriptions will be represented in the coming sections.

\subsubsection{Background}

If one counts the number of transition of some states (e.g. a lithology) to the others in an equidistance traverse and put them in a matrix, it will be called a transition frequency matrix.

Dividing the row totals of the transition frequency matrix by the total number of transitions (the complete sum of transition numbers), the relative proportions of the states will be obtained. The corresponding matrix is called marginal (or fixed) probability vector (Davis 2002, p. 170).

In many geological investigations, data sequences may be seen that consist of the ordered successions of mutually exclusive states. For instance, measured stratigraphic sections have the form of series of lithologies, where the type of lithology or in a drill-hole through an ore body, the ore and gangue can be considered as states. Observations along a traverse may be taken at equally spaced intervals or they may be taken wherever a change in state occurs (embedded Markov chains). Sometimes the nature of the transitions from one state to another is of main interest rather than the relative locations of the states in a sequence Davis 2002, p. 168).

According to the conditional probability equations (Davis 2002, p. 170), if the probability of the occurrence of the states A and B are independent, the probability of occurring state $\mathrm{B}$ at a point given the state $\mathrm{A}$, equals to the probability of the occurrence of the state $\mathrm{B}$ :

$$
\operatorname{Pr}(\mathrm{B} \mid \mathrm{A})=\frac{\operatorname{Pr}(B, A)}{\operatorname{Pr}(A)}=\frac{\operatorname{Pr}(B \cap A)}{\operatorname{Pr}(A)}=\frac{\operatorname{Pr}(B) \cdot \operatorname{Pr}(A)}{\operatorname{Pr}(A)}=\operatorname{Pr}(\mathrm{B})
$$


This allows the prediction of, for example, what the transition probability matrix should look like if the occurrence of a lithologic state at one point in the stratigraphic interval were completely independent of the lithology at the immediately underlying point. The expected transition probability matrix would consist of rows that were all identical to the fixed probability vector.

For comparing this expected transition probability matrix with the actually observed one to test the hypothesis that all lithologic states are independent of immediately preceding states, one can use $\chi^{2}$ test. For this purpose, first the probabilities should be converted to expected numbers of occurrences by multiplying each row by their corresponding total number of occurrences and the expected and observed should be compared values by $\chi^{2}$ criterion.

\subsubsection{Transition probability-based indicator geostatistics}

\section{(1) Objectives and background}

As mentioned earlier, because a big part of the geological data is categorical (for example, lithofacies, soil classes, mineralization phases and so on), indicator kriging becomes increasingly popular for modeling them. Moreover, some continuous parameters may represent non-Gaussian behavior which necessitates the use of non-parametric approaches. However, modeling the spatial continuity which can be considered the most crucial and difficult practical step in applying the indicator kriging method, may face serious problems when sufficiently abundant data does not exist. Due to the lack of enough data in such cases, subjective and interpretative information can fill this gap to make reliable models. In transition-probability indicator geostatisticsal methods, proportions, mean lengths (e.g. mean thicknesses), and juxtapositioning patterns (i.e. how a category locates in space with respect to another category) are used as subjective information to improve modeling spatial continuity (Carle and Fogg 1996, pp. 453-454).

\section{(a) Some definitions and relations:}

The cross-variogram $\gamma_{m k}(\mathrm{~h})$ of the indicator variables is defined as:

$$
2 \gamma_{m k}(\mathrm{~h})=\mathrm{E}\left\{\left[\mathrm{I}_{m}(\mathrm{x})-\mathrm{I}_{m}(\mathrm{x}+\mathrm{h})\right]\left[\mathrm{I}_{k}(\mathrm{x})-\mathrm{I}_{k}(\mathrm{x}+\mathrm{h})\right]\right\}
$$


where $\gamma_{m k}(\mathrm{~h})$ denotes the indicator cross-variogram between the categories $\mathrm{m}$ and $\mathrm{k}, \mathrm{I}_{m}$ $(\mathrm{x})$ and $\mathrm{I}_{k}(\mathrm{x})$ are the indicators of the category $\mathrm{m}$ and $\mathrm{k}$ in location $\mathrm{x}$, respectively., and $\mathrm{h}$ is the lag separation vector (Carle and Fogg 1996, pp. 455). The equation is defined using the mathematical expectation function.

The cross-covariance $\mathrm{C}_{m k}(\mathrm{~h})$ is defined as (Carle and Fogg 1996, pp. 455):

$$
\mathrm{C}_{m k}(\mathrm{~h})=\mathrm{E}\left\{\mathrm{I}_{m}(\mathrm{X}) \mathrm{I}_{k}(\mathrm{x}+\mathrm{h})\right\}-\mathrm{E}\left\{\mathrm{I}_{m}(\mathrm{x})\right\} \mathrm{E}\left\{\mathrm{I}_{k}(\mathrm{x}+\mathrm{h})\right\}
$$

where $\mathrm{C}_{m k}(\mathrm{~h})$ is the cross-covariogram between the categories $\mathrm{m}$ and $\mathrm{k}$. As applied to measuring spatial continuity, the transition probability $\mathrm{t}_{m k}(\mathrm{~h})$ from category $\mathrm{m}$ to category $k$, is defined in the form $\mathrm{f}$ a conditional probability function as (Carle and Fogg 1996, pp. 455):

$$
\mathrm{t}_{m k}(\mathrm{~h})=\operatorname{Pr}\{\text { category } k \text { occurs at } \mathrm{x}+\mathrm{h} \mid \text { category m occurs at } \mathrm{x}\}
$$

Transition-probabilities are geologically easier to interpret than variograms and covariograms (Elfeki and Dekking 2001, pp. 569).

The indicator (cross-) variogram, indicator (cross-) covariance, and transition probability are related to each other as different combinations of one-location marginal probabilities $\mathrm{p}_{k}(\mathrm{x})$ defined as (Carle and Fogg 1996, pp. 455):

$$
\mathrm{p}_{k}(\mathrm{x})=\operatorname{Pr}\left\{\mathrm{I}_{k}(\mathrm{x})=1\right\}=\mathrm{E}\left\{\mathrm{I}_{k}(\mathrm{x})\right\}
$$

and two-location joint probabilities $\mathrm{p}_{m k}(\mathrm{x}, \mathrm{h})$ defined as (Carle and Fogg 1996, pp. 455):

$$
\mathrm{p}_{m k}(\mathrm{x}, \mathrm{h})=\operatorname{Pr}\left\{\mathrm{I}_{m}(\mathrm{x})=1 \text { and } \mathrm{I}_{k}(\mathrm{x}+\mathrm{h})=1\right\}=\mathrm{E}\left\{\mathrm{I}_{m}(\mathrm{x}) \mathrm{I}_{k}(\mathrm{x}+\mathrm{h})\right\}
$$

or (Carle and Fogg 1996, pp. 455):

$$
\mathrm{t}_{m k}(\mathrm{~h})=\operatorname{Pr}\left\{\mathrm{I}_{k}(\mathrm{x}+\mathrm{h})=1 \mid \mathrm{I}_{m}(\mathrm{x})=1\right\}=\operatorname{Pr}\left\{\mathrm{I}_{k}(\mathrm{x}+\mathrm{h})=1 \text { and } \mathrm{I}_{m}(\mathrm{x})=1\right\} / \operatorname{Pr}\left\{\mathrm{I}_{m}(\mathrm{x})=1\right\}
$$

Typically in practice, the assumption of a stationary model removes the dependence on the location $\mathrm{x}$ so that:

$$
\mathrm{E}\left\{\mathrm{p}_{k}(\mathrm{x})\right\}=\mathrm{p}_{k} \quad \forall \mathrm{x} \in \mathrm{D}
$$

In which $\mathrm{p}_{k}$ denotes a constant (Carle and Fogg 1996, pp. 455) and

$$
\mathrm{E}\left\{\mathrm{p}_{m k}(\mathrm{x}, \mathrm{h})\right\}=\mathrm{p}_{k}(\mathrm{~h}) \quad \forall \mathrm{x} \in \mathrm{D}
$$

where $\mathrm{p}_{m k}(\mathrm{x}, \mathrm{h})$ denotes a joint probability depending only on lag $\mathrm{h}$. 
Passing the detailed theories, it can be summarized that the cross-variograms and crosscovariograms can be expressed in terms of the transition-probabilities and proportion, as following (Carle and Fogg 1996, pp. 456):

$$
\begin{aligned}
& \gamma_{m k}(\mathrm{~h})=\mathrm{p}_{m}\left\{\mathrm{t}_{m k}(0)-\left[\mathrm{t}_{m k}(\mathrm{~h})-\mathrm{t}_{m k}(-\mathrm{h})\right] / 2\right\} \\
& \mathrm{C}_{m k}(\mathrm{~h})=\mathrm{p}_{m}\left[\mathrm{t}_{m k}(\mathrm{~h})-\mathrm{p}_{k}\right]
\end{aligned}
$$

In general, it can be demonstrated that the indicator kriging method can be reformulated using the transition-probabilities.

\section{(b) Methodology}

First, two key points regarding the transition probability-based indicator geostatistical technique deserve mentioning:

(a) Geological and subjective information can be taken into account to improve the modeling of the spatial continuity of the indicator geostatistical simulation in the framework of the transition-probabilities indicator geostatistics.

(b) All kriging equations can be reformulated in terms of the transition-probabilities among the existing soil categories.

Therefore, the method can be performed through the following steps:

1. Estimating and inferring various pieces of subjective information such as proportions, mean length and juxtapositioning relations.

2. Calculating all the transitions probabilities of the samples between pairs of the classes (auto- and cross-transitions).

3. Modeling the transitions probabilities using Markov chain models.

4. Solving the related equations and performing the geostatistical simulation.

Carle and Fogg (1996) have discussed this reformulation by detail and represented that the normal indicator cokriging equation are equivalent to: 


$$
\left[\begin{array}{cccc}
t_{m l}\left(x_{1}-x_{1}\right) & \ldots & t_{m l}\left(x_{N}-x_{1}\right) & I_{m l} \\
\cdot & \cdot & \cdot & \cdot \\
\cdot & \cdot & \cdot & \cdot \\
\cdot & \cdot & \cdot & \cdot \\
t_{m l}\left(x_{1}-x_{N}\right) & \ldots & t_{m l}\left(x_{N}-x_{N}\right) & I_{m l} \\
I_{m l} & \ldots & I_{m l} & 0
\end{array}\right]\left[\begin{array}{c}
\lambda_{l k, 1} \\
\cdot \\
\cdot \\
\cdot \\
\lambda_{l k, N} \\
\mu_{l k}
\end{array}\right]=\left[\begin{array}{c}
t_{m k}\left(x-x_{1}\right) \\
\cdot \\
\cdot \\
\cdot \\
t_{m k}\left(x-x_{N}\right) \\
I_{m k}
\end{array}\right]
$$

(Carle and Fogg 1996, p. 468) and the unbiasedness condition can be expressed as :

$$
\sum_{\alpha=1}^{N} \sum_{l=1}^{K} p_{l} \lambda_{l k, \alpha}=p_{k}
$$

in which $t_{m l}\left(x_{i}-x_{j}\right)$ is the transition probability of transition from class $m$ to class $l$ for the separation vector of $\left(x_{i}-x_{j}\right), I_{m l}$ is the identity matrix, $\lambda_{l k, 1} \ldots \lambda_{l, N}$ are cokriging weights pertaining to the transition of the soil categories from 1 to $\mathrm{k}$ considering the $\mathrm{N}$ nearby estimation points, $p_{l}$ and $p_{k}$ are the proportion of the classes 1 and $\mathrm{k}$, and finally $\mu_{l k}$ denotes the $K \times K$ matrix of the Lagrange parameters (Carle and Fogg 1996, p. 468).

\section{(2) Taking the subjective and geological information into account:}

Subjective information such as the proportions, mean lengths, and juxtapositioning relations can be integrated into the simulation models to improve the modeling of the spatial continuity. Following, there are some practical points to integrating the mentioned information in the spatial variability modeling which have been explained briefly here:

\section{(a) Proportions:}

Proportions can be deduced directly from the indicator data and conceptual models. Proportions can be helpful in fitting the sill of a spatial continuity model, whether expressed in terms of covariogram $\left(\mathrm{C}_{m k}(\mathrm{~h})\right)$, cross-variogram $\left(\gamma_{m k}(\mathrm{~h})\right)$, or transitionprobabilities $\left(\mathrm{t}_{m k}(\mathrm{~h})\right)$. Equally, the sill of a spatial continuity model entails the guessed and estimated proportions. Thus, the relationship between proportions and the sill is connected to subjective development and abstract understanding of the spatial continuity model. 
One might be interested in verifying whether or not the sill of a spatial variability model is acceptable? The frequency of the observations might not be so sufficient to provide an adequate to make a proper decision about the sill of the spatial variability model so easily. Conversely, proportions can also be inferred from the sills.

The sill of $t_{m k}(h)$ comes near $\mathrm{p}_{k}$, the proportion of category $k$, whether $m=k$ or $\mathrm{m} \neq \mathrm{k}$. On the contrary, the sills of $\gamma_{k k}(\mathrm{~h})$ and $\mathrm{C}_{k k}(0)$ come close to $p_{k}\left(1-p_{k}\right)$, which requires solving for "' $p$ "' in a quadratic equation of sill $=p(1-p)$. Then the question would be about $p_{k}$ whether $p_{k}$ equals $p_{k}$ or 1 - $\mathrm{p}$ ? The sill of $\gamma_{m k}$ (h) and $\mathrm{C}_{m k}(0)$ for $\mathrm{m} \neq \mathrm{k}$ approaches $-p_{m} p_{k}$, an even more ambiguous situation.

The stress on the relationship between proportions and model sills is not to determine proportions from bivariate statistics (e.g. transition-probabilities), instead to check for consistency of the spatial continuity model with proportions established by univariate data and conceptual information. In subjective model fitting of the sill, prior information on proportions (usually exist) can be applied as a guide for either $\mathrm{C}_{m k}(\mathrm{~h}), \gamma_{m k}(\mathrm{~h})$, or $\mathrm{t}_{m k}$. Obviously, among the mentioned measures, $\mathrm{t}_{m k}(\mathrm{~h})$ provides the straightest relationship between the model sill and proportions.

\section{(b) Mean Length:}

The slope at origin of the graphs of: $\mathrm{t}_{k k}\left(\mathrm{~h}_{\phi}\right), \gamma_{k k}\left(\mathrm{~h}_{\phi}\right)$ and $\mathrm{C}_{k k}\left(\mathrm{~h}_{\phi}\right)$ is directly related to mean length (mean thickness) of the soil category $k$ in the direction $\phi$.

Supposing stationarity for proportions, the mean length of the category $k$ in a direction $\phi$, represented by $\mathrm{l}_{k, \phi}$, relates to the slope at the origin of $\gamma_{k k}\left(\mathrm{~h}_{\phi}\right), \mathrm{C}_{k k}\left(\mathrm{~h}_{\phi}\right)$ and $\mathrm{t}_{k k}\left(\mathrm{~h}_{\phi}\right)$ by:

$$
\begin{gathered}
\frac{\partial \gamma_{k k}\left(h_{\phi} \rightarrow 0\right)}{\partial h_{\phi}}=\frac{p_{k}}{\bar{l}_{k, \phi}} \\
-\frac{\partial C_{k k}\left(h_{\phi} \rightarrow 0\right)}{\partial h_{\phi}}=\frac{p_{k}}{\bar{l}_{k, \phi}} \\
-\frac{\partial t_{k k}\left(h_{\phi} \rightarrow 0\right)}{\partial h_{\phi}}=\frac{1}{\bar{l}_{k, \phi}}
\end{gathered}
$$


Obviously, mean lengths can be computed directly from continuous data, but not from discontinuous data.

\section{(c) Juxtapositioning patterns:}

As mentioned before, "juxtapositioning" patterns refer to how one category in the space is located preferentially or non-preferentially in relation to the other one, including nonrandom and directional-specific (asymmetric patterns) like cycles of fining upwards. Considering the fact that transition-probabilities are asymmetric, i.e. $\mathrm{t}_{m k}(\mathrm{~h}) \neq \mathrm{t}_{k m}(\mathrm{~h})$, these patterns also will be reflected in the estimation stage.

An important point in the indictor geostatistics is to ensure that the models conform to the basic rules of the probability. For example, the estimated probabilities should not be negative or greater than one, or the estimated probabilities from the indicator (co)kriging should sum up to one.

\section{(3) Advantages and disadvantages of TP/MC method}

Before summarizing the advantages and disadvantages of the TP/MC method, it deserves mentioning that the literature review for different Markov chain methods revealed an interesting point. Each author usually had emphasized on the pros of own method without clarifying its cons. However, a next person who proposed a new method usually criticized the disadvantages of the previous methods and emphasized again on the advantages of his own method without mentioning the shortcomings of own technique. Therefore, the drawbacks of the previous methods could be found in different papers mentioned by the next authors.

The advantages of transition probability-based indicator geostatistics (TP/MC) over traditional indicator geostatistical estimation and simulation can be summarized as:

(a) Easier full consideration of the auto- and cross-correlations structure between pairs of the categories.

(b) Easier integrations of geological subjective information of facies architecture in modeling spatial continuity (Carle 1999).

(c) More consistency with order relation rules.

(d) Taking the asymmetry in the spatial variability structure of the transitionprobabilities into account to construct more geologically plausible models. 
While the indicator cross-variograms can not disclose the asymmetry (Carle and Fogg 1996, p. 463).

(e) In general, in the methods based on Markov chains, the intrinsic non-stationarity of heterogeneities in larger scales is represented much well (Park 2010, p. 1).

On the other hand, because the TP/MC method follows the same framework as the indicator geostatistics, it still faces some similar shortcomings as the indicator geostatistics, for instance:

(a) As represented before, the first stage of the TP/MC method is based on the reformulation of conventional indicator (co)kriging equations. Therefore, the order-relation violations can still exist.

(b) Laborious model fitting and a rather long simulation course, because this method does not apply the Markov approach directly. Specially, the quenching or optimization stage of the TP/MC simulation to lessen the difference between the transition probability structures to their corresponding models can be considerably time-consuming.

\section{(4) Why the TP/MC method was chosen:}

In spite of the mentioned disadvantages of the TP/MC method, among the Markov chainbased techniques reviewed in literature review stage of the present study, i.e. coupled Markov chain (CMC) and Transition-probability geostatistics (TPG), the later techniques still had several theoretical and practical drawbacks. For instance, they were not suited sufficiently for three-dimensional problems and they still did not have available computer codes or software to use. They were also prone to producing artifacts.

Hence, it was decided to apply the TP/MC scheme while some attempts were made to improve this technique.

\section{(5) Software:}

T-PROGS developed by Steven F. Carle (1999) is a package which enables the user to apply the transition-probability/Markov approach to geostatistical simulation of categorical variables. This free package is developed in FORTRAN programming framework and can be requested from the author. 


\section{Transition Probability}

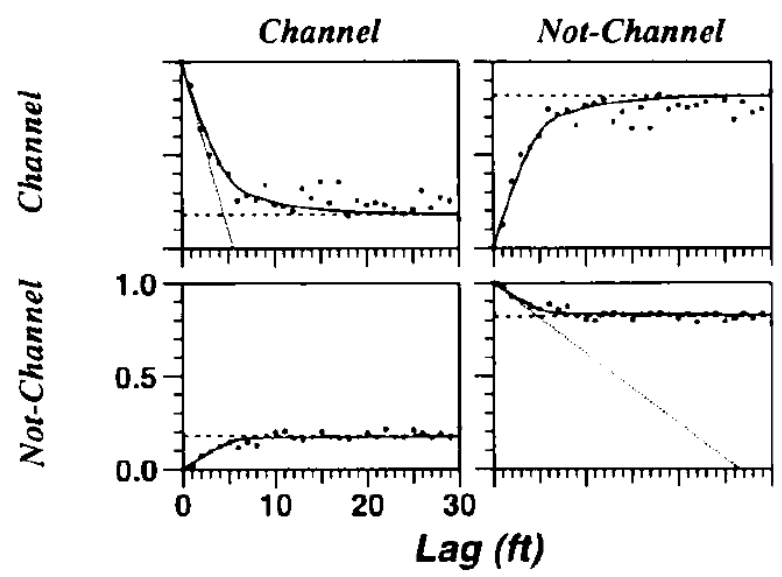

Figure 5.2 an example of (auto- and cross-) transition-probabilities, and relevant Markov chain models for a two-category facies model of Channel and Not-Channel. Points represent the observed values, solid curves represent the Markov chain models, dashed-lines stand for the proportions, and slopes show the estimated slopes from the mean lengths. The graph is taken from Carle and Fogg (1996, p. 459).

Moreover, a software called GMS, distributed by EMS-I, is a package for modeling purposes especially for groundwater problems which provides a MS Windows, interface to T-PRGOS.

\subsubsection{The modeling stages in the TP/MC technique}

The modeling stages using the TP/MC method has already been explained in the previous sections.

In this part, some more details are brought about the modeling steps.

Practically, the following were steps taken in this study for simulation of the soil categories:

(a) Calculating sample transitional-probabilities (i.e. transitions against separation distance) between pairs of the existing classes in various directions.

(b) Modeling the mentioned transition-probabilities using Markov chain models. Length statistics can also be considered in this stage to improve the models by integrating more subjective information or to verify the inferred Markov chain models. Modeling the transition-probabilities by Markov chain models can be conducted through different methods. It can be proven that, all the Markov chain models of the transition- 
probabilities can be constructed using an exponential matrix form like Equation (5-1). During the modeling of the transition-probabilities using the Markov chain models, some constraints and conditions should be checked. For example, the auto-transition rates should be always negative while the cross-transition rates should be positive. The row-sums and column-sums of the transition rate matrix should obey the Equations (533 ) and (5-34). The models can be checked for their conformity to the length statistics (Equations (5-17) and (5-26)), and the general tendencies of the categories to occur beside or above each other (juxtapositioning patterns and the fining or coarsening upward or downwards). More detailed discussions about various modeling methods of the transition-probabilities by Markov chain models are mentioned in the coming sections.

(c) The Figure 5.3 and

(d) Figure 5.4 represent the calculated and model transition-probabilities among geotechnical soil classes for horizontal and vertical directions. In this study, although the observations were considerable and there were no vital need to integrate the subjective information, the conformity of the Markov chain models of the transitionprobabilities with subjective and interpretive information were also considered. In the cases where the subjective information were considerably different from their inferred values from the Markov chain models, the models were adjusted to achieve models which were more consistent with geological information.

(e) Conducting the simulations using the mentioned Markov chain models and simulation framework. The simulations here were based on the reformulated indicator (co)kriging equations using transition-probabilities and Markov chain models.

(f) Further optimization stages (simulated annealing or quenching stages) could be done using some objective functions. The objective function for performing the simulated annealing in TP/MC method is defined based on the closeness of the calculated transition-probabilities of the simulation results to the target Markov chain models of them. Therefore, in the quenching step of this simulation method, the deviation of the initial simulation and the target (Markov chain) model is minimized in terms of the transition-probabilities of the categories, under specific convergence conditions, to achieve a new improved simulation result. This result should represent a more acceptable spatial structure in terms of the transition-probabilities reproduction. The quenching usually improves the geological soundness of the simulated models. In this 
study, as it will be shown later, quenching improved the geostatistical /statistical and geological soundness of the simulation results.

\subsubsection{Markov chain models of transition-probabilities}

Spatial Markov chains, as explained before, stand for a change model in states (e.g. a sequence of lithofacies in a specific direction) so that the state in a point is dependent solely on the nearest points but not the others. This spatial Markov chain can be described based on the spatial probability of changing each state, e.g. a soil type, to the others socalled transition-probabilities that are dependent only on the separation vector of points and not the location in the stationary case.

A one-dimensional spatial Markov chain model supposes that the result at the specific location depends fully on the closest datum. A three-dimensional spatial Markov chain model conveys that the spatial variability in any one direction can be described by a onedimensional Markov chain model (Carle 1999, p. 25).

Even though the Markov chain, in theoretical and mathematical view, is identified very simply, it has demonstrated considerable applicability in spatial variability characterization of facies or hydrostratigraphic units in alluvial and fluvial depositional systems (Carle 1999, p. 28).

Mathematically, it can be shown that the Markov chain consists of linear combinations of exponential structures, although non-exponential-looking 'Gaussian'” and 'hole-effect" structures can also be generated.

The Markov chain model in the $\varphi$ direction can be fully formulated using an exponential matrix equation like this:

$$
T\left(h_{\varphi}\right)=\exp \left(R_{\varphi} h_{\varphi}\right)
$$

and therefore the Markov chain models can be fully defined having the transition rates. The transition rates are the change of a transition probability from one state to another in unit length.

In this equation, $h_{\varphi}$ denotes a lag in the direction $\varphi$, and $R_{\varphi}$ denotes a transition rate matrix: 


$$
R_{\varphi}=\left[\begin{array}{cccc}
r_{11, \varphi} & r_{12, \varphi} & \ldots & r_{1 K, \varphi} \\
r_{21, \varphi} & r_{21, \varphi} & \ldots & r_{2 K, \varphi} \\
\vdots & \vdots & \ddots & \vdots \\
r_{K 1, \varphi} & r_{K 2, \varphi} & \ldots & r_{K K, \varphi}
\end{array}\right]
$$

where $r_{j k, \varphi}$ stands for changing rate from category $j$ to category $k$ for each unit length in direction $\varphi$.

Attention should be paid that the Equation (5-18) do not reflect a plain mathematical exponential equation but it conveys an eigenvalue analysis procedure since the matrix exponential is not calculated solely by computing the exponential of matrix elements, that is, $t_{j k, \varphi}\left(h_{\varphi}\right) \neq \exp \left(r_{j k, \varphi} h_{\varphi}\right)$.

Symbolizing $R_{\varphi}$ and $h_{\varphi}$ by $\mathrm{R}$ and $\mathrm{h}$, respectively, for notational simplification, (Rh) can either be approximated by an infinite series or even preferably with an exact form of:

$$
\exp (R h)=\sum_{i=1}^{K} \exp \left(\lambda_{i} h\right) Z_{i}
$$

where $\lambda_{i}$ and $Z_{i}$ represent the eigenvalues and spectral component matrixes of $\mathrm{R}$ for $\mathrm{K}$ categories, respectively.

To define the term of "eigenvalue" and to keep the mathematical accuracy, the sentences have been exactly quoted here from Weisstein, E. W. "Eigenvalue." -- from Wolfram MathWorld, A Wolfram Web Resource (accessed in fall 2011):

"Eigenvalues are a special set of scalars associated with a linear system of equations (i.e., a matrix equation) known also as characteristic roots, characteristics values (Hoffman and Kunze 1971), proper values, or latent roots (Marcus and Minc 1988, p. 144)".

"The determination of eigenvalues and eigenvectors of a system is extremely important issues in physics and engineering.

Each eigenvalue is paired with a corresponding so-called eigenvector.

If $A$ is a linear transformation represented by a matrix $A, \lambda$ is called the eigenvalue of $A$ with a corresponding vector $X \in R^{n} \neq 0$, if there is such a vector $X$ so that:

$$
A X=\lambda X ”
$$

The mathematical details are discussed in the relevant literature (e.g. Agterberg (1974) and Carle and Fogg (1997) and Carle and others (1998)). 
An eigenvalue, like $\lambda_{i}$, is inherently zero and is related to a spectral component matrix having the proportions along each column. Thus, for a four-category system, the continuous lag Markov chain model written out completely consists of

$$
\begin{aligned}
& \exp (R h)=(1.0)\left[\begin{array}{llll}
p_{1} & p_{2} & p_{3} & p_{3} \\
p_{1} & p_{2} & p_{3} & p_{3} \\
p_{1} & p_{2} & p_{3} & p_{3} \\
p_{1} & p_{2} & p_{3} & p_{3}
\end{array}\right]+\exp \left(\lambda_{2} h\right)\left[\begin{array}{llll}
z_{11,2} & z_{12,2} & z_{13,2} & z_{14,2} \\
z_{21,2} & z_{22,2} & z_{23,2} & z_{24,2} \\
z_{31,2} & z_{32,2} & z_{33,2} & z_{34,2} \\
z_{41,2} & z_{42,2} & z_{43,2} & z_{44,2}
\end{array}\right] \\
& +\exp \left(\lambda_{3} h\right)\left[\begin{array}{llll}
z_{11,3} & z_{12,3} & z_{13,3} & z_{14,3} \\
z_{21,3} & z_{22,3} & z_{23,3} & z_{24,3} \\
z_{31,3} & z_{32,3} & z_{33,3} & z_{34,3} \\
z_{41,3} & z_{42,3} & z_{43,3} & z_{44,3}
\end{array}\right]+\exp \left(\lambda_{4} h\right)\left[\begin{array}{llll}
z_{11,4} & z_{12,4} & z_{13,4} & z_{14,4} \\
z_{21,4} & z_{22,4} & z_{23,4} & z_{24,4} \\
z_{31,4} & z_{32,4} & z_{33,4} & z_{34,4} \\
z_{41,4} & z_{42,4} & z_{43,4} & z_{44,4}
\end{array}\right]
\end{aligned}
$$

In which, the $z_{j k, i}$ are the coefficients of the spectral components matrices $Z_{i}$ calculated by the eigensystem analysis (Carle 1999, p. 30).

Hence, the Markov chain model for every element of $t_{i j}(h)$ in $T(h)$ equals to a linear combination of the K-1 exponential categories plus the column category proportion. For a four-category case, this can be expressed as following (Carle 1999, p. 30):

$$
t_{i j}(h)=p_{j}+z_{i j, 2} \exp \left(\lambda_{2} h\right)+z_{i j, 3} \exp \left(\lambda_{3} h\right)+z_{i j, 4} \exp \left(\lambda_{4} h\right)
$$

The Markov chain model for in TP/MC method can be established using different methods such as; discrete lag Markov chain method, maximum entropy, transition frequencies, embedded transition frequencies, and embedded transition-probabilities.

"The lateral extent of the 3-DMarkov chain model output by MCMOD" must be finite, with limits that consider statistical closeness. Kriging-based algorithms, which do not consider cross-correlations, easily rank statistical closeness by the magnitude of the variogram (or covariance) model or a prescribed search radius with anisotropy ratios. However, the ranking of a full cross-correlation matrix for multiple categories is not so straightforwa"(Carle 1999, p. 30).

The determinant as a closeness measure is used not only in the MCMOD program of the T-PROGS software to rank the statistical closeness in MCMOD program but it is also

\footnotetext{
${ }^{1}$ A program in T-PROGS software to find a proper Markov chain model for transition-probabilities.
} 
used in the search and simulated quenching algorithms of the TSIM program (in the TPROGS software).

It can be shown that the determinant of the transition-probability matrix $T(h)$ would be the product of its eigenvalues $\theta_{k}(h)($ Carle 1999, p. 30):

$$
\operatorname{det} \mathbf{\Gamma}(h) \equiv \prod_{k=1}^{K} \theta_{k}(h)
$$

More details about the eignsystem analysis can be found in relevant literature (e.g. Carle 1999, pp. 27-28).

Two- or three-dimensional Markov chain models can be achieved by accepting the assumption that the spatial variability in every direction can be characterized using a 1-D Markov chain model. The 1-D Markov chain models in each direction can be achieved by interpolating the 1-D Markov chain models in principal direction models. Similar conclusion can be made about the transition rates based on the assumption that the transition probability matrix can be fully determined by the use of the transition rates matrix (Carle 1999, p 30).

A brief description of each Markov chain transition probability modeling method is explained following:

\section{(a) Transition rates:}

When the transition rates, which equal to the slopes of the transition-probabilities at the origin, can be inferred directly (for example from the transition probability graphs of the input data) or from subjective information such as mean lengths and interpretations of the facies successions, this technique could become a suitable choice.

In addition, in the case that the rough initial transition rates are inferable from another Markov chain modeling (e.g. discrete-lag method) and analysis method, the transition rates can be adjusted and fine-tuned to achieve better fits in the next stages.

\section{(b) Discrete-lag Markov chain model:}

If a transition probability matrix $T\left(\Delta h_{\varphi}\right)$ is multiplied successively by itself at lag $\Delta h_{\varphi}$, the Markov chain model can be formulated using the discrete-lag method.

It can be shown that, the transition-rates matrix can be formulated as following, in this technique (Carle 1999, p. 27): 


$$
R_{\varphi}=\frac{\ln \prod\left(\Delta h_{\varphi}\right)}{\Delta h_{\varphi}}
$$

which contains the eigensystem analysis. More details about the eigensystem analysis can be found in the relevant literature.

Therefore, one can use this method to produce the Markov chain models that honor the calculated transition-probabilities from input data in specific unit lags, for example, in one-, two-, three-, or n-unit lags.

This method is usually applied in the initial steps of Markov-chain modeling to obtain a rough estimation of the models and transition rates.

In this case, the Markov-chain models of transition-probabilities were initially made based on discrete-lag method. Different lags were to tested to find the model that fits the best to all of the observed transition rates while fulfills the statistical requirements of the model such as negative auto-transition rates and positive cross-transition rates. Checking the debugging file, it could be evaluated whether or not the models had statistical problems and in the necessary cases, there were modified.

In this study, in most cases, a 3-lag (discrete-lag) Markov chain model was considered as the initial transition rates Matrix approximation step.

\section{(c) Transition-probabilities of embedded Markov chain analysis:}

Embedded transition-probabilities are defined based on the probabilities of the transitions among the categories where the states are changed. Therefore, there are no autotransitions, in embedded Markov chain analysis.

Embedded Markov chain analysis provides the most interpretive framework in Markov chain modeling. Therefore, it can be applied in the case of sparse data or even no data (Carle 1999, pp. 35-36).

For example, the relation of the embedded transition-probabilities, mean length and transition rates can be expressed as following:

$$
r_{i j, z}=\frac{\pi_{i j, z}}{L_{i, z}}
$$

where $r_{i j, z}$ stands for the transition rate from category $\mathrm{i}$ to category $\mathrm{j}, \pi_{i j, z}$ is the embedded transition probability from category $\mathrm{i}$ to category $\mathrm{j}$, and $\bar{L}_{i, z}$ indicates the mean length of the category $i$, all in vertical direction.

In addition, the following condition also should be met: 


$$
\sum_{j=1}^{K} \pi_{i j, z}=1
$$

in which, $\mathrm{K}$ is the total number of categories.

In addition, interpretations and subjective information, inferred from embedded transition-probabilities, can be used to check and verify the chosen Markov chain models. The embedded transition-probabilities, stored in the debugging file of the MCMOD program of T-PROGS software, can be interpreted for some subjective information such as the tendency of categories to occur above or beside each other and the mean length of each category in specific directions for example in vertical direction that would be the mean thickness of each category.

In general, a category with the highest frequency is considered in T-PROGS software as the background category. Besides, since in the embedded transition-probabilities analysis there are no auto-transitions, the inference of embedded transition-probabilities is not too difficult by integrating subjective information in Markov chain modeling of the transitionprobabilities.

\section{(d) Transition frequencies of embedded Markov chain analysis:}

In an embedded Markov chain transition-probabilities analysis, the numbers of transitions are indicated at the first step. For example, the count of transitions from soil class 1 to the soil class 2 and to all other classes are determined to calculate the transition-probabilities from class 1 to all other classes. Here, again, there are no auto-transitions. With normalizing the matrix of the number of counts by the sum of the whole matrix, the transition frequencies are calculated. If these counts are normalized by the row totals, the transition-probabilities will be achieved. The application of transition frequencies rather than transition-probabilities is suitable in the evaluations of statistical independence.

\section{(e) "Independent" or "maximum entropy" (disorder) Markov chain analysis:}

If there are no tendencies in the occurrence of any category over or beside the others, the conditions of the "independence" or maximum entropy should be met.

"The disorder of the juxtapositional tendencies in a particular direction, say $\varphi$, can be

quantified by the entropy $S_{\varphi}$ of bed-to-bed transition frequencies $f_{j k, \varphi}$, the probabilities that one bed occurs next to another, by": 


$$
S_{\varphi}=-\sum_{j} \sum_{k} f_{j k, \varphi} \ln \left(f_{j k, \varphi}\right)
$$

It can be proved that in the Maximum-entropy conditions, the transition rates can be obtained by:

$$
r_{j k, z}^{S(\text { Max })}=\frac{f_{j k, z}^{S(\text { Max })}}{\overline{L_{j, z}} \sum_{k \neq j} f_{j k, z}}
$$

\subsubsection{TP/MC simulation technique}

TP/MC simulation is performed in two stages:

(a) The initialization stage:

In this step, a method similar to the sequential indicator simulation (SISIM) algorithm (explained by Deutsch and Journel 1998, pp. 125-127, p. 149-150) is applied, except that this technique uses a transition probability-based indicator cokriging to approximate the local conditional probabilities:

$$
\operatorname{Pr}\left\{\mathrm{k} \text { occurs at } x_{0} \mid i_{j}\left(x_{\alpha}\right) ; \alpha=1,2 \ldots N, j=1,2 . . K\right\} \approx \sum_{\alpha=1}^{N} \sum_{j=1}^{K} i_{j}\left(x_{\alpha}\right) w_{j k, \alpha}
$$

In above equation (Equation (5-30)), the parameters definitions of the equation can be as summarized following:

$\mathrm{N}$ : data number in the neighborhood, i: indicator for category $\mathrm{j}, w_{j k, \alpha}$ : estimation weights for transition from the state $\mathrm{j}$ to the state $\mathrm{k}$, and $\alpha$ is the index of the points applied in the estimation of the conditional local probabilities (Carle 1999, p. 47).

Estimation weights $\left(w_{j k, \alpha}\right)$ can be calculated from solving Equations (5-13) and (5-14). The only point is that, in the mentioned equations, estimation weights have been denoted as $\lambda$ instead of $w$.

Implementation of transition probability-based cokriging, improves the consideration of spatial interclass cross-correlations. 
(b) Simulated annealing:

Simulated annealing or quenching is an optimization stage. A more detailed description of simulated annealing method for geostatistical analyses can be found in Deutsch 2002 (pp. 275-294) as well as in Deustch and Cockerham 1994. In this stage, initially generated configurations using a transition probability-based SISIM technique, are improved and optimized to enhance the agreement between measured and modeled transitionprobabilities. In other words, the difference between the Markov chain models of transition-probabilities and the calculated transition-probabilities of the simulations are decreased.

Thus the attempt in quenching step is to solve the optimization problem of

$$
\left.\min \left\{O=\sum_{l=1}^{K} \sum_{j=1}^{K} \sum_{k=1}^{K} \mathbf{l}_{i k}\left(h_{l}\right)_{S I M}-t_{j k}\left(h_{l}\right)_{\text {MOD }}^{2}\right]\right\}
$$

where $\mathrm{O}$ denotes the objective function, $h_{l}$ represents the $1=1, \ldots, \mathrm{M}$ indicated lagvectors, $\mathrm{j}$ and $\mathrm{k}$ show the $\mathrm{K}$ existing categories, and finally "SIM" and "MOD" distinguish the simulated (measured from the realization) and model transitionprobabilities, respectively (Carle 1999, p. 53).

In each simulated point, the neighbouring states are cycled and the transition-probabilities are calculated. An objective function is defined as following:

$$
\mathrm{O}=\mathrm{E}\left\{(\text { Transition probability of simulation- Transition probability of model })^{2}\right\}
$$

If the perturbation minimizes the objective function $(\mathrm{O})$, it is accepted and otherwise it is rejected.

In this case, considering the mentioned soil classification scheme, due to rather enough amounts of data-points, considering the length statistics has no significant effect on improving the Markov chain models, although in fewer data, it would be more effective and useful for sure. The discrete-lag method and inferred proportions were first used to make the MC models, they were checked for statistical accordance, and then the models were further examined using the length statistics. 


\subsection{Transition-probability Markov chain (TP/MC) geostatistical modeling of geotechnical data-set in Göttingen test site}

\subsubsection{Overview:}

To perform a transition-probability Markov chain geostatistical simulation and analyses of geotechnical soil-classes model in Göttingen test zone, the first step, as argued before, was to calculate the transition-probabilities among different classes in various directions from input data. Similar to the sequential indicator simulation (SISIM) and indicator kriging (IK) modeling methods, the transformed coordinates system was applied in transition probability Markov chain-based analyses. These transition-probabilities (autoand cross-transitions) were calculated and plotted for horizontal (Omni-directional horizontal) and vertical directions.

The next step was to find a suitable Markov chain model that not only fit the transitionprobabilities of the input data but also it could fulfill some basic criterions. These criterions have been summarized following:

(1) The auto-transition rates should be minus.

(2) The off-diagonal transition rates should not be negative.

(3) The row sums for transition rates matrix should be zero (Carle 1999, p. 29):

$$
\sum_{k=1}^{K} r_{j k, \phi}=0
$$

(4) The column sums, considering the proportions of the categories, must conform the following equation (Carle 1999, p. 29):

$$
\sum_{j=1}^{K} p_{j} r_{j k, \phi}=0
$$

(5) The transition rate values should individually be reasonable values.

All the mentioned criterions are checked internally in the MCMOD program of $\mathrm{T}$ PROGS software and reflected in a debugging file.

The first step in finding the proper Markov chain model which could fulfill the mentioned criterions and fit the sample transition-probabilities acceptably started with discrete-lag Markov chain modeling. In discrete-lag Markov chain method, the model 
honors the transition-probability data in a specific (discrete) lag. In other words, the model passes through a transition probability point in a specific lag, e.g. second-lag of transition probability plot, which has been calculated from the sample data.

One can choose the first-, second-, third- or a bigger number-unit lag transition probability point depending on the spatial structure of data which produces the best fit while no errors in MCMOD debugging file.

As Carle and Fogg (1997) have suggested, the Walther's law of facies succession (Leeder 1982, p. 122) can be applied as a practical means to infer the horizontal transition-probabilities structure of a sedimentary succession from its vertical-direction structure and vice versa. Walther's law of facies succession (in the absence of nonconformities) implies that the vertical succession of sedimentary environments reflects their lateral structure (Leeder 1982, p. 122).

Hence, an observed fining upward asymmetry in the vertical transition-probabilities might be incorporated to construct the fining outward model in the dip-direction transition rates (Carle and Fogg 1997, p. 908).

The next step usually is to improve the Markov chain model using embedded Markov chain method. In the embedded Markov chain modeling where the embedded transitions are considered, the length statistics and other interpretive information can also be taken into account. Embedded transition probability Markov chain modeling method is in general more flexible and interpretive. In the case of this study, the Markov chain modeling of transition-probabilities started with discrete-lag modeling (3-lag), followed by embedded Markov chain method and finally using the transition rates to improve the models using the adjustment of transition rates.

The produced models in MCMOD program were checked by plotting the data transitionprobabilities and Markov chain models in the same graph. Figure 5.3 and

Figure 5.4 represent the data transition-probabilities and the chosen calculated models for vertical and horizontal directions.

In the MCMOD program the operator defines the Markov chain model type, categories proportions and the relevant model parameters as well as input data file. Then the program produces five output files as the elements of Markov chain model. Three files are the transition-probabilities of Markov chain models in $\mathrm{x}, \mathrm{y}$, and $\mathrm{z}$ directions respectively. These files are used in plotting and checking the produced Markov chain models compared to the transition probability of the input data. These three files have the ASCII file format. Therefore, the strategy for checking the models is to plot the transition- 
probabilities of model(s) and those of data in a same plot and comparing them together as mentioned above. Then, the debugging files were checked for their conformity to the required conditions. The graphs always do not change significantly but the debugging file may show better conditions with required fine-tuning of the Markov chain models to fulfill the necessary conditions.

The two other files which include the three-dimensional Markov chain model are in binary format. One of the two mentioned files is the three-dimensional model file and the second file is determinant file which contains the statistical closeness data (see Section 5.1.4 for more details about the method).

After choosing, adjusting and improving the three-dimensional Markov chain transition probability models, regarding the debugging file of MCMOD program and transition probability graphs, one can also perform some length statistics checks to improve or verify the existing model, especially in the case of insufficient data. In the case of lack of enough information, the length statistics can play an important role to fill the information gap in order to approximate the proper Markov chain models or improve them.

In this study, due to the existence of sufficient samples and data, the application of length statistics did not seem to be necessary. 


\section{Geotechnical classes Goettingen soils Vt. Transition Probabilities}

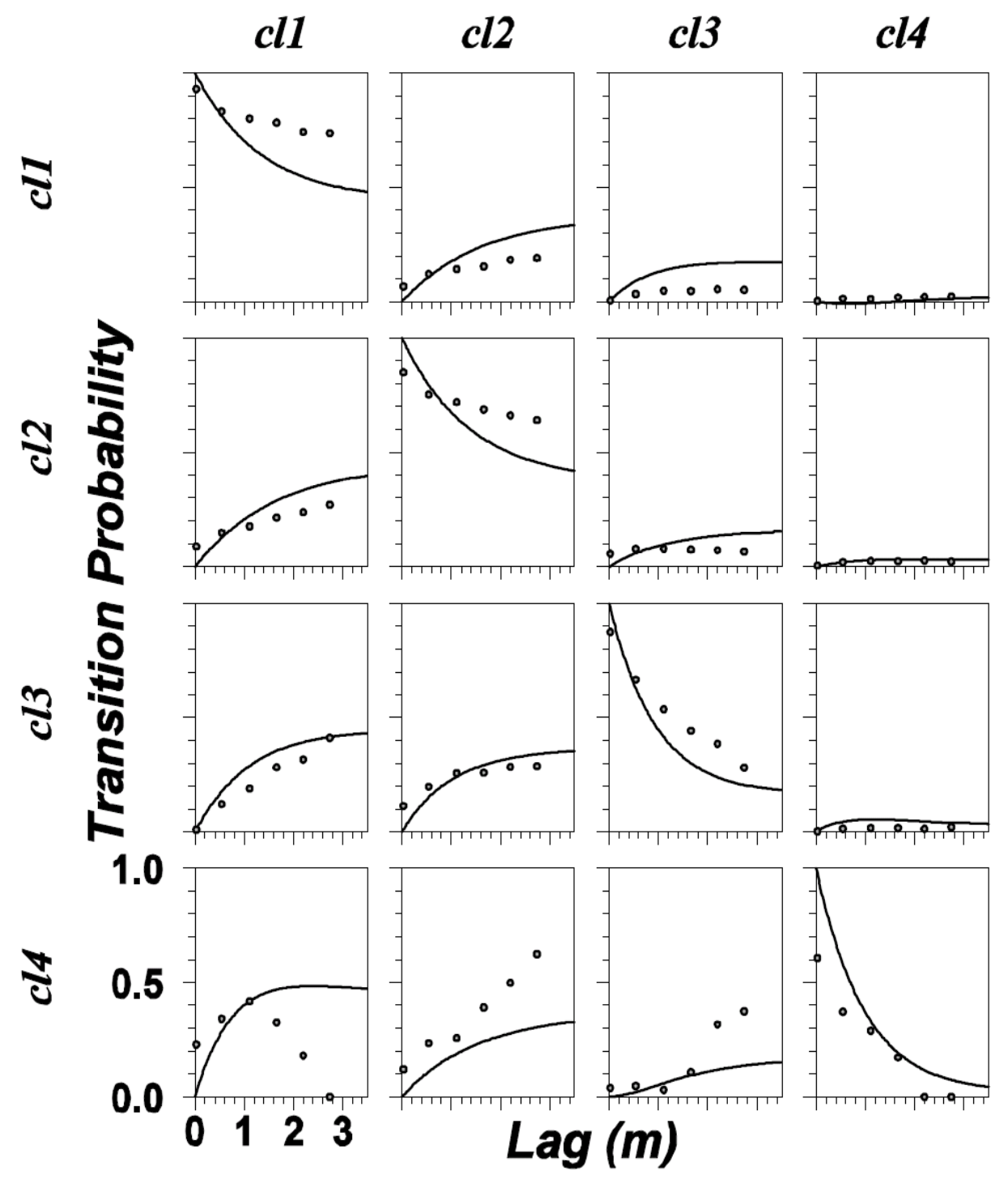

Data

Rates MC Model

Figure 5.3 Transition-probabilities of the four geotechnical soil categories in vertical direction calculated from input data (dots) and their corresponding Markov chain model (solid lines) from transition rates method as a final fine-tuned model. 


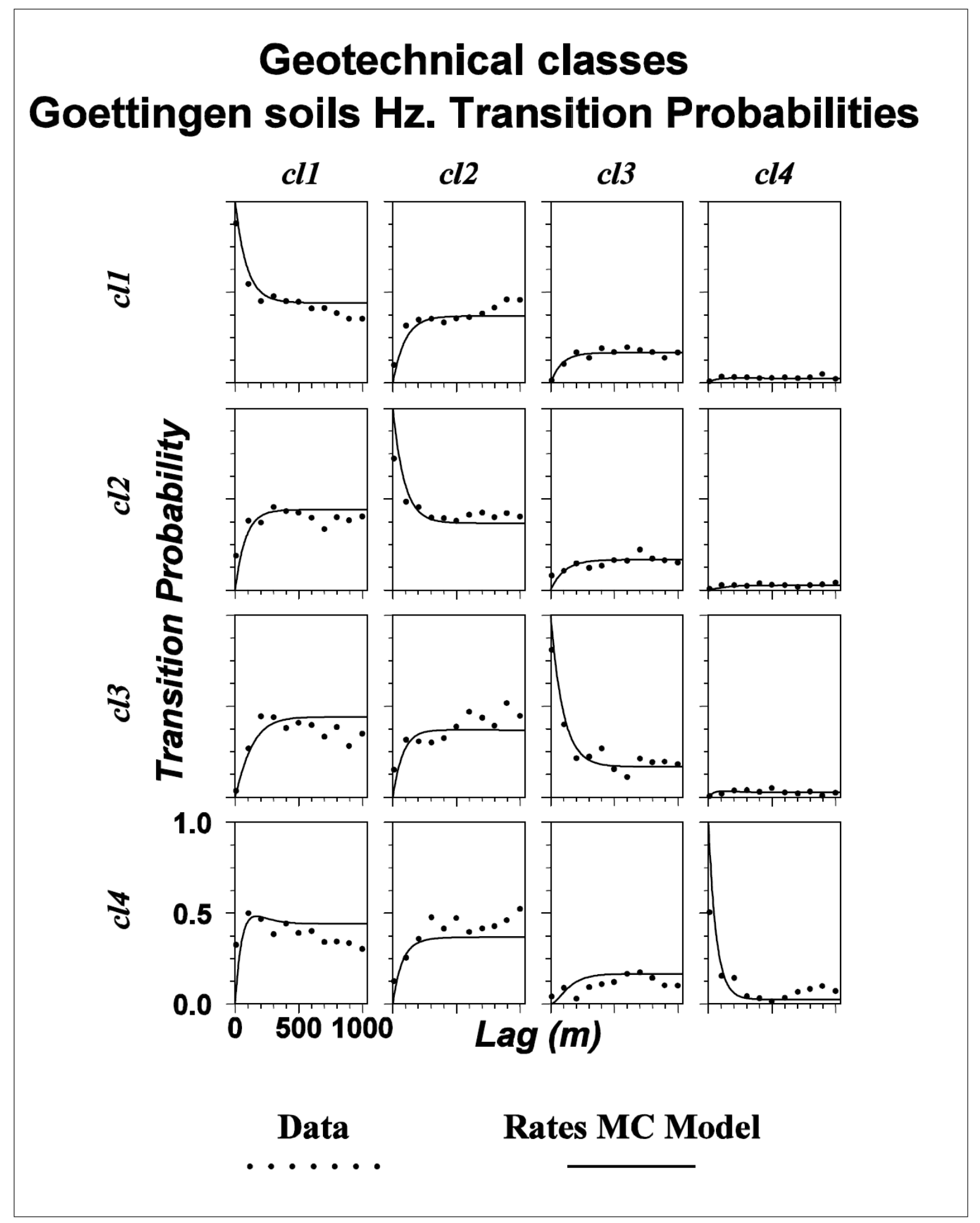

Figure 5.4 Transition-probabilities of the four geotechnical soil categories in horizontal direction calculated from input data (dots) and their corresponding Markov chain model (solid lines) from transition rates method as a final fine-tuned model. 


\subsubsection{Some points about using post-quenching phase in TSIM program of the T-PROGS software:}

To perform the TP/MC simulations, termed here as TSIM simulation, the TSIM program of the T-PROGS software was applied. There were some practical points and considerations in the applications of this program in the present study which has not been explained here to avoid too much discussions and details. In this section, a summary of the most essential points of these points have been addressed.

For the reasoned explained before, the TSIM simulations started with generating simulations without quenching steps. Then, every further quenching step was performed on the available simulations of the previous stage. However, the TSIM program conducts these quenching steps on the same output file of the available simulations from previous stage(s) by assigning a negative number for the quenching steps. Therefore, the existing simulation as well as output files (which give a summary of each simulation round) had to be copied first and renamed for the next stage. Copying the simulation files was for preserving the existing simulation results and renaming was for applying the last simulation results for the next quenching step with different names to avoid replacing the existing files. Since, the simulations were recorded in different files but there was only one generic "tsim.out" file for each round, the proper renaming had to be done on the output files in each step. To make it also easier to calculate the variograms of all the TSIM, simulation, the realizations of each TSIM simulation were merged into one file with compatible format of the GSLIB software. Scripting was applied during this study to make several handlings and calculations easier or even feasible.

\subsection{Evaluating the TP/MC simulation results and their underlying models}

\subsubsection{Overview:}

To evaluate the simulations produced by transition probability-based Markov chain method, some interpretive and statistical criterions were employed and suggested in this study.

The purpose and meaning of the produced simulations evaluations and their underlying models was to assess the goodness of applied models for TP/MC simulations, improve 
them if necessary, and suggest the best produced realizations in terms of mentioned criteria. In addition, the proper criterions were sought to compare the results out of different geostatistical simulation methods and evaluate how reliable in general these criterions were.

Oy Leuangthong et al (2004, p. 72) have summarized the minimum acceptance criteria for geostatistical realizations. Based on their suggestions, the following criteria to evaluate the qualities of produced simulations and choosing the best ones have been considered in this study:

(a) Honoring the input (i.e. conditioning) data at their locations.

(b) Histogram (or proportions) reproduction.

(c) Transition probability Markov chain models reproduction (as a measure for spatial variability structure).

(d) Variogram reproduction (as another spatial structure measure).

(e) Geological soundness of the produced model.

Actually, the criterion of TPs-reproduction has not been suggested by Oy Leuangthong et al (2004). However in this study, the application of TPs-reproduction as another spatial variability measure has been suggested to improve the evaluation of the spatial variability structure, especially to evaluate the interclass transition structures. In particular, TPsreproduction criterion could match better to the TP/MC method which essentially relies on modeling the TPs as spatial variability structure measure and using Markov chain models.

More details about and the results of each mentioned criterion have been explained further, in the coming sections.

\subsubsection{Honoring the input (conditioning) data values at their locations or data reproduction:}

It is a known fact that (co-)kriging is an exact estimator, so that the estimate at a data location equals exactly to the input data value. In other words, if kriging estimation is performed at the location of a data point, the result would exactly be the same as input data value at that location and the estimation variance will be zero:

$$
z_{k}^{*}=z\left(u_{\alpha}\right), \quad \forall u=u_{\alpha}, \quad \alpha=1, \ldots, n
$$


where $z_{k}^{*}$ represents the kriging estimation value of the random variable, $\mathrm{Z}$, at location $\mathrm{u}$ and $z\left(u_{\alpha}\right)$ stands for the data value at location $u_{\alpha}$.

Considering that initial stage of the applied simulation methods was based on reformulated (co-)kriged estimations and their corresponding estimation variances to define the local conditional cumulative distribution functions (CCDF), the applied simulation methods also had to reproduce the data exactly at their locations. However, in practice, for some reasons, few data points were not reproduced. For example, in some simulation algorithms that assign data points to the simulation grid nodes, in order to speed up distance calculations, some input data may not be reproduced. For that reason, the total number of assigned data may become less than the total number of available conditioning data. This phenomenon can happen probably because of one of the following reasons:

1. The sample coordinate may be situated outside of the limits of threedimensional model (simulation) grid (Figure 5.5, A).

2. The data point may be inside the limits of 3D simulation grid, but it is trimmed or there is no variable available (Figure 5.5B).

3. If there are more than one data points available inside the 3D simulation grid limits near to a simulation grid block and one of samples is closer to the block center, the closer sample will be kept and the other sample will be ignored (Figure 5.5, C).

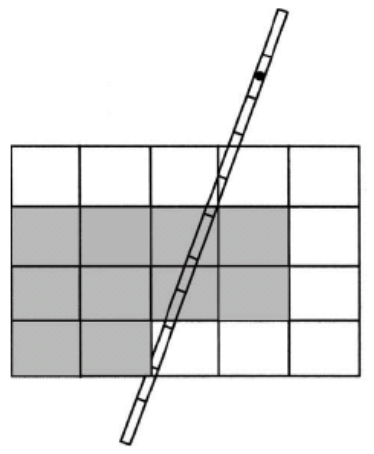

A Outside 3-D grid.

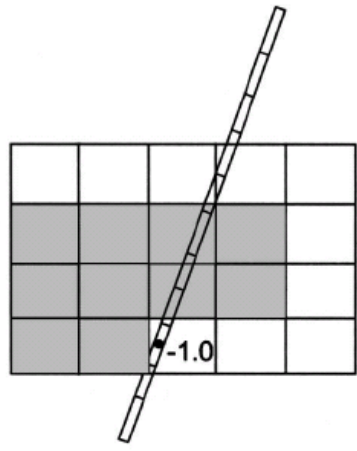

B In 3-D Grid, but no value.

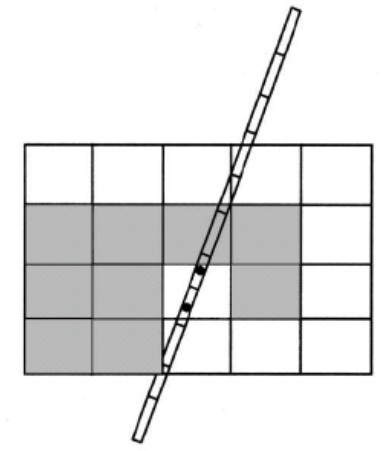

C In 3-D grid, but multiple data in one block.

Figure 5.5 a schematic illustration of how the input data may not be assigned to the simulation grid node: $A$, the sample exists but it is located outside the simulation grid; $B$, the existing sample is inside the simulation grid but the value is trimmed; and $\mathrm{C}$, samples exist and are inside the simulation grid but there are more than one samples inside the grid block but only the closest sample is assigned (the graph is taken from Leuangthong (2004, p. 133). 
In WinGslib software, the user can decide to let or not let the SISIM program to assign the data to the model nodes. The assigning of the data to the simulation model nodes can be chosen to reduce the simulation run-time. In this case, the decision was to let the SISIM program of WinGslib software to assign the data to the simulation nodes to speed up the simulation procedure. In addition, some data points could also be trimmed in the SISIM program. Therefore, it was expected that a number of input data points would not be reproduced (Leuangthong 2004, p. 133).

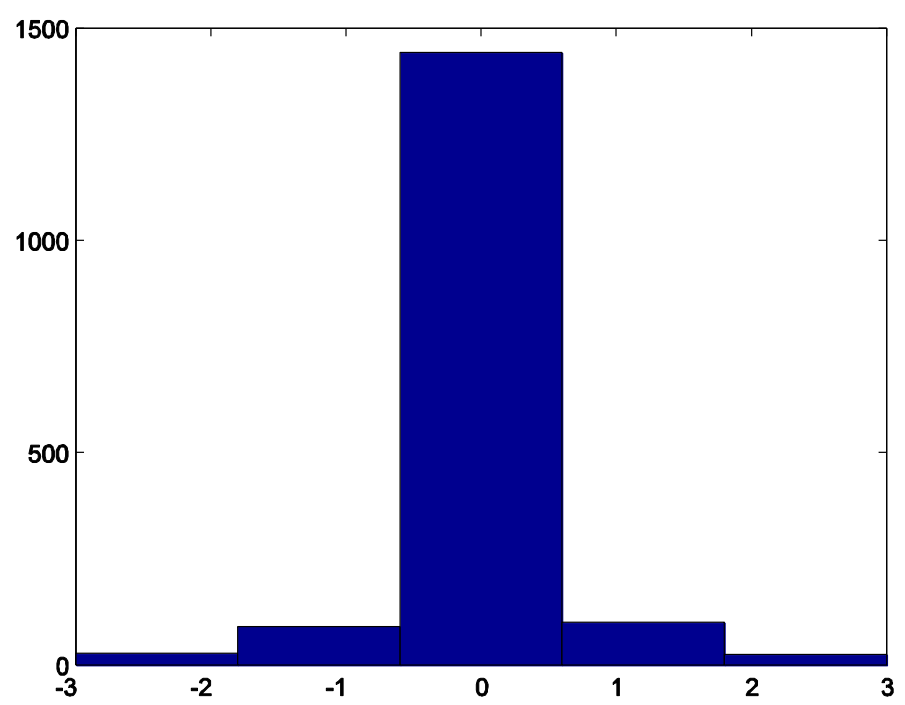

Figure 5.6 input data conditioning errors histogram of SISIM simulation method for realization number 20.

Input data reproduction can be checked in different ways. Oy Leuangthong et al (2004, pp. 133-134) have suggested the use of cross-plots in order to assess the goodness of input data reproduction. However, since this approach is not well-suited for categorical attributes, another solution was adopted here to facilitate the evaluation of conditioning data reproduction for each realization. A MATLAB code was developed to compare the observed and simulated soil categories and assess the conditioning-data reproduction ratio in all sample points in the simulation models. This check was performed for all realizations generated by various simulation methods. For this purpose, in each step the mentioned MATLAB code reads the soil category of a data point from borehole data file and finds the soil category in its nearest simulation point of a realization from its corresponding simulation file. Then, the code compares the observed and simulated categories from these two points with calculating their differences. Finally, the ratio of the correctly conditioned simulation points in each realization (with the difference values of 
zero), was calculated for all realizations. The MATLAB output was copied and stored in a text file separately for each simulation method. The conditioning data reproduction can be evaluated afterwards. This difference also could be represented in a conditioning-errors histogram. The error then is the difference of the observed category values of wellbore data and their corresponding simulated category in their nearest points. Ideally, this histogram should only contain zero values (100\% of the frequency) that would mean the exact reproduction of all input (conditioning) data points. In practice, however, some errors are expected. Figure 5.6 represents an input data conditioning error histogram of the realization number 20 for the SISIM simulation method. The histogram is quite symmetric with a governing frequency on no errors, meaning that the input data reproduction was almost perfect except for a minor portion of data points. Some errors in this method also could occur due to the imperfect match of the simulation and data points. For instance, if a data point is located between two simulation points with exactly similar distances from data point, the computer code will consider the first point in the simulation file.

In simulation number 20 of the SISIM method, the $99.64 \%$ of input data was exactly reproduced.

The results show that the input data reproduction of the SISIM realizations were in the range of $99.47 \%$ and $99.88 \%$ that is nearly perfect for all realizations. The same checks were performed on the realizations of different TSIM methods.

The conditioning on input data for the TSIM method is also about $89.47 \%$ to $95.22 \%$ which seems also fairly acceptable but weaker compared to the SISIM method.

Since it was not possible to represent all the conditioning-errors histograms of all the realizations of different simulation methods, the qualities of input data reproduction was evaluated solely with the ratio of exactly conditioned simulation points in which the conditioning-errors were exactly zero.

Table 5.1 summarizes the simulations conditioning on the input data for different simulation methods.

The clear conclusion from this table could be that, the conditioning-data (input data) reproduction can be accepted for the realizations of all utilized simulation methods in this study. However, the conditioning on the input data has clearly been reduced by adding the number of quenching steps. Since the reduction of the input data honoring with further quenching steps is unfavorable whereas the extra quenching steps increase the other statistical and geological qualities of the simulations, a compromising decision could be 
made to keep a good input data reproduction as well as an acceptable simulation at the same time. Therefore, it can be suggested that the number of quenching steps should not be too much but at the same time it should not also be too few.

Table 5.1 The table represents a summary of honoring (conditioning on input data) for various geostatistical simulation methods.

\begin{tabular}{|c|c|c|c|c|}
\hline $\begin{array}{l}\text { Simulation method } \\
\text { Conditioned input data }\end{array}$ & Statistic & SISIM & $\begin{array}{l}\text { TSIM without } \\
\text { any quenching } \\
\text { steps }\end{array}$ & $\begin{array}{l}\text { TSIM with two } \\
\text { quenching } \\
\text { steps }\end{array}$ \\
\hline \multirow{4}{*}{$\begin{array}{l}\text { Based on the all input data } \\
\text { points }\end{array}$} & Minimum & 0.9947 & 0.923 & 0.8863 \\
\hline & Maximum & 0.9988 & 0.9431 & 0.9277 \\
\hline & $\begin{array}{l}\text { Arithmetic } \\
\text { mean }\end{array}$ & 0.9979 & 0.9329 & 0.9103 \\
\hline & $\begin{array}{l}\text { Satadard } \\
\text { deviation }\end{array}$ & 0.0011 & 0.0064 & 0.0120 \\
\hline \multirow{4}{*}{$\begin{array}{l}\text { Based on the accepted } \\
\text { input data points in the } \\
\text { simulation program }\end{array}$} & Minimum & 0.9947 & 0.9318 & 0.8947 \\
\hline & Maximum & 0.9988 & 0.9522 & 0.9366 \\
\hline & $\begin{array}{l}\text { Arithmetic } \\
\text { mean }\end{array}$ & 0.9979 & 0.9418 & 0.9190 \\
\hline & $\begin{array}{l}\text { Satadard } \\
\text { deviation }\end{array}$ & 0.0011 & 0.0064 & 0.0121 \\
\hline
\end{tabular}

\subsubsection{Histogram- (or proportions-) reproduction:}

\section{(1) Introduction:}

One of the least requirements for a realization of a geostatistical simulation to become acceptable, in statistical sense, is its ability to reproduce its expected representative 
histogram of the attributes under study. The target attribute in this study was the soil categories. Hence, a realization that, its histogram of the studied attributes, deviates too much (beyond an acceptable tolerance) from the target distribution, is rejected for histogram reproduction. In such cases, either another realization that fulfills this criterion better can be selected for further evaluations or the underlying models of the simulation should be adjusted to achieve a more suitable set of results which reproduce the target histogram better. Even, it could also be necessary to choose a different simulation method to achieve more proper simulation results that better reproduces the expected histogram. It should be reminded here that, the primary geostatistical models in this study were initially improved, prior to the geostatistical simulations. For the kriging-based method of SISIM, the cross-validation, and for the transition-probability-based method of TSIM, the Markov chain model parameters control was applied to improve the initial models which were implemented later for geostatistical simulations. About the transition-probabilitybased methods, the debugging files were checked for the accordance of the simulation models statistics to the necessary statistical conditions such as non-negative crosstransition and negative auto-transition rates in the transition-rates matrix. More details about the necessary conditions of the TP/MC simulation models have already been discussed in the relevant section.

Two essential points should be kept in view when evaluating the quality of histogram reproduction for a realization of a geostatistical simulation:

(a) A number of proper criterions should be chosen to verify the closeness of the histograms of the soil categories in realizations to that of the reality as well as to assess how close these distributions are and which realization reproduces the real distribution better.

(b) The practical limitations and suitability as well as available software should be considered

The application of the Q-Q plots could be considered as one of the most common techniques in histogram reproduction evaluations of geostatistical realizations of a simulation method. A Q-Q plot, in fact, compares the distributions of the two sample groups. For instance, in the case of histogram reproduction tests of the geostatistical simulations, the realizations versus the expected (target) distributions can be compared with each other. This method is especially suitable to be implemented when evaluating all the realizations at once. In such cases, the Q-Q plot of all realizations versus the input 
data is plotted to compare the distributions of these two sets. If the Q-Q plot (roughly) follow the 45-degree line of the $\mathrm{y}=\mathrm{x}$, it means that the two distributions, i.e. the simulations and the expected one in this case, are almost identical. Still, this technique is better suited for the continuous data-sets rather than the categorical ones and especially in assessing all the realizations at once. Besides, the calculation and plotting of such huge point-sets, due to a high computational demand, is rarely feasible. For example, in this application, the GSLIB and R programs exceeded their (memory and run-time) limits. Thus, the application of Q-Q plots was not preferred for the proportions-reproduction test in this project. Consequently, an analytical alternative was preferred for testing the histogram reproduction of such a categorical attribute, particularly because of offering a clearer index to evaluate and compare the results by such an analytical measure.

To approximate the target distribution, in this study, the application of the volumetric proportions of the categories inferred from the borehole data considering the vertical grids-transformation corrections could be applied. This suggestion was supported by similar cases in the literature such as Ritzi et al. (2007) as well as in Deutsch and Journel (1998), and the discussion in Section 2.1.6. To achieve a modified representative histogram of the soil categories, the effect of the grids transformation in vertical direction had to be considered. In addition, due to the existence of some clustering in boreholes locations, corrections such as cell- or polygonal-declustering seemed necessary. The declus program in GSLIB software can be used for a cell-declustering of the input data. Both the mentioned methods including the declustering and direct proportions calculations from boreholes data with corrections for vertical grids-transformation were applied separately here to estimate the representative global proportions. Then, the results of these two methods were compared with each other. Nevertheless, there are still three important points about these corrections:

(a) The applied declustering algorithm depends very much on declustering parameters that affect the global histogram approximation.

(b) When these two methods to estimate the global histogram were applied separately, the results out of them were almost equal. As stated before, the first method was cell-declustering technique and the second approach was the volumetric proportion calculation from the borehole data just with considering the correcting weights proportional to the effective-range of samples in vertical directions in the transformed space. Therefore, in the second technique, the weight of each sample in the calculation of the proportions in global distribution was 
higher in the thinner parts of the layer where there were less number of samples, and lower where the layer was thicker and the number of samples was more. According to the conducted cell-declustering of input data, the global proportions of the soil categories from two methods were more or less similar.

(c) When performing the ordinary kriging algorithms, the declustering is not necessary because the ordinary kriging algorithm, automatically and effectively corrects the clustering effect (Deutsch 2002, p. 50). However, for the geostatistical simulations, a representative histogram in which the clustering problem has been modified is greatly necessary.

In this study, as mentioned above, the estimated global distribution of soil categories calculated by declustering method and from input data with vertical grid-transformation corrections were not considerably different. In addition, the declustering parameters were also affecting the estimated histogram. Therefore, the application of the second method which was easier and more consistent has been suggested for similar cases. Yet, the locally varying proportions and the proportions estimation uncertainties as well as the conceptual or deterministic process-based models or the secondary information such as seismic measurements could also be taken into account for a better proportion estimation of soil categories. The reason of the suitability of the second method, i.e. inferred from boreholes data with corrections related to the grid-transformation, for the proportions (of categories) estimation, was due to the plenty of available samples as well as the lack of significant trend in the proportions variations over the study area in the transformed-grid space.

Hence, in this study, a realization was not rejected for the histogram reproduction when its proportions of the categories were close-enough to those of the expected ones (considering the acceptable tolerances). This evaluation had to be clearly supported by statistical concepts. Moreover, another insight to the proportions reproduction evaluation could be that both boreholes data and simulations should reflect the same populations which closely imitate the distribution of existing categories in the reality, all in the transformed space. Taking such an insight into consideration for this problem, the chisquare statistical homogeneity of populations test was also suggested to assess the proportions reproduction in this case. 


\section{(2) The proportions-reproduction criteria:}

Considering the above discussion, two decisive factors were suggested in this study to evaluate the proportions-reproductions of the realizations of each simulation method; first, a chi-square test for homogeneity of populations, and second, the deviation rate of the observed from expected proportions together with a chi-square-like statistic that will be explained more in the coming sections which has been named here as sum of the rates of squared deviations. This measure has been defined according to the squared deviation of the observed from expected proportion relative to the expected proportion which is defined similar to the definition of the normalized mean square deviation statistic. In addition, the chi-square $\left(\chi^{2}\right)$ statistic for homogeneity of proportions test was also applied to assess the proportions-reproduction in the realizations and rank them in terms of its goodness. The ranking of the realizations in terms of the chi-square statistic for the quality of proportions reproduction, was based on the fact that, the lower the $\chi^{2}$ value, the closer the distribution of that realization should be to the expectation, because the $\chi^{2}$ statistic reflects the departure of the observed frequencies from the expected ones. There are also further statistical measures for the evaluation of the accordance of the realizations and target (real) distributions in a simulation method, such as KolmogrovSmirnov goodness of fit test for the continuous parameters or the RMS (root mean square) statistic for the categorical data (e.g. Clausen (1982, p. 55) for RMS).

\section{a. Proportions-reproduction check using a deviation rate framework:}

To evaluate the quality of the proportions-reproduction, a criterion has been suggested here which has been referred as the proportions "deviation rate" (D.R. or DR). This rate seems comparable to the framework of the mean absolute percentage error (MAPE) or mean absolute percentage deviation (MADE). However, the difference between these two methods is that, the DR is evaluated for each category separately and the greatest emphasize is considered on the most dominant classes. MADE is applied in evaluation of the model fitting in time series. The deviation rate of the proportion of category $i$ in a realization, has been defined in terms of the ratio of the deviation of the proportion of a category (i.e. category $i$, in this case) from the expected (i.e. global) proportion:

$$
D . R_{\cdot_{i}}=\frac{\left|\left(P_{O}\right)_{i}-\left(P_{E}\right)_{i}\right|}{\left(P_{E}\right)_{i}}
$$


where $D . R_{\cdot_{i}}$, for a realization of a geostatistical simulation, is the deviation rate of the observed proportion of the class $\mathrm{i}$ from its expected proportion, $\left(P_{O}\right)_{i}$ is the observed soil category proportion of the class $\mathrm{i}$ in the mentioned realization, and $\left(P_{E}\right)_{i}$ is the expected proportion of the same (global proportions).

It should be emphasized again that the proportions of the soil categories inferred from the boreholes, taking the grid transformation corrections into account, were regarded as the target distribution in the "deviation rate" calculation here.

In the proposed framework, the deviation rate of the proportions of soil categories (D.R. ) for each realization of a simulation method was evaluated, starting the evaluation from the proportion of the soil category with the highest observed frequency in the boreholes (after necessary modifications related to the grid-transformations) and ending to the least frequent soil category from input data. Hence, a more emphasize was put on a class with the highest expected frequency and then, the second highest expectedly frequent class, and so forth. The practical steps of this method were as following:

(a) First of all, the proportions of soil categories in the realizations were extracted from simulation output files and put in a table that besides, included the expected proportions of the categories.

(b) Next, the deviation rates for each category were calculated using the above formula (5-36).

(c) In the third step, the deviation rates which were not greater than the maximum acceptance limit, e.g. $0.1(=10 \%)$ or $0.05(=5 \%)$, were determined and indicated. The key classes for evaluation of the deviation rates of the proportions were the two most, and three most expectedly dominant classes in the SISIM (classes 2 and 1), and TSIM (classes 2, 1, and 3) methods, respectively. The maximum accepted deviation rates were $0.1(10 \%)$ for the SISIM method and $0.0025(0.25 \%)$, or at times, 005 (5\%), for the TSIM methods. The reason for considering a much lower acceptable deviation rate limit for the TSIM methods were because of much closer proportions to those of the target in the realizations of the TSIM methods. The signification of the acceptable deviation rates were by assigning a "1" when it was acceptable and a " 0 " otherwise.

(d) Then, the realizations representing the acceptable deviation rates for the first two, or in some cases, first three expectedly most dominant soil classes were also indicated. 
(e) The indicated realizations pointed out in step (4) were selected as realizations with acceptable proportions reproduction.

(f) These selected realizations were sorted then, in terms of a statistic which looks like a chi-square statistic but has been defined somewhat differently and has been named in this study as the sum of the rates of squared deviations (SRSD). As mentioned before, this measure has been defined according to the squared deviation of the observed from expected proportions relative to the expected proportion. Actually, this measure has been defined similar to the coefficient of the variation of the mean square deviation statistic or the chi-square statistic. The difference of this measure with the coefficient of the variation of the mean square deviation is that there is no averaging in SRSD and the normalization is done against the expected values for each squared deviation but not the total mean of the observed (proportion) values. In this sense, SRSD is more similar to the chi-square statistic. However, the observed and expected values should be in terms of counts in the chisquare statistic while the observed and expected proportions are employed in SRSD. Therefore, the SRSD was defined (proposed) in this study in somewhere between the chi-square and the coefficient of the variation of the mean square deviation. The aim of defining such a measure was to bring a complementary measure to highlight the absolute differences (by squaring the deviation) relative to the expected value (by dividing by its expected value) and summing these normalized deviations. However, there is no standard table for this statistic and it can only be used to compare among the different simulations to rank them for their quality of proportions-reproduction. The analytical definition of the SRSD has been brought in the next lines. The selected realizations were sorted in terms of their SRSD rates statistic of the proportions in an ascending order. The best realizations in terms of the proportions-reproduction should represent the least sum of the SRSD value as an indicator for the closeness of the simulation proportions to those from corrected (for the grid transformations in the vertical direction) observations in the boreholes.

Hence, wherever these deviation rates in a realization, for the two or three first expectedly most dominant classes, were in an acceptable range, let's say, 0.1 or 0.05 , the related realization was accepted and otherwise declined.

For instance, if the two classes that expected to be most predominant soil categories were considered as a basis to evaluate the quality of proportions-reproduction, let's say, class $j$ and class $k$ (which were respectively classes 2 and 1 in this research) and the maximum 
acceptable deviation rate is $D . R_{a}$, then the acceptance criterion could be expressed as following:

$\left\{\left(D . R_{\cdot_{j}}<D . R_{\cdot_{a}}\right)\right.$ and $\left.\left(D . R_{\cdot_{k}}<D . R_{\cdot_{a}}\right)\right\} \Rightarrow$ the realization is acceptable in terms of the quality of proportions reproduction.

The reasons for suggesting such a framework were:

(a) Methods that could not be implemented or were not adequate: The Q-Q plot was not applicable in such cases not only due to software limitations but also for its incompatibility to the purpose of the categorical attributes evaluations. Moreover, the chi-square test of homogeneity does not always seem capable of identifying a satisfactory proportions-reproduction adequately. As an example, regarding the expected proportions of the categories (i.e. the target distribution), the $X^{2}$ statistic (as a calculated estimation of the $\chi^{2}$ statistic) for the homogeneity of proportions test suggested the realization number 20 as the best proportions-reproducing realization for the TSIM-without-quenching-step simulation method, whereas the calculated proportions of the realizations $16^{1}$ and $12^{2}$, putting more emphasize on the most dominantly observed classes in the input data, have obviously closer distributions to that of the target. In part $(b)$, it will be explained why the belief here was that putting more stress on the most anticipated classes was a sensible decision. It has already been explained why the proportions of the soil categories inferred from the input borehole data were regarded a proper estimation of the real ones in this case. In addition, the chi-square method is not straightforward in terms of the departures from the expected proportions. Actually, a better decision could be made with a criterion that expresses these deviations more directly, for example in terms of a deviation rate. Moreover, the chi-square statistic for test of homogeneity, usually, gives a single overall value for all proportions-reproduction elements (proportionsreproduction of cl1 to cl4). Nevertheless, one can also evaluate each single chisquare element for each soil category separately, to compare the expected and realization proportions.

\footnotetext{
${ }^{1}$ The proportions of the soil classes 1 to 4 for the realization 16 in TSIM method without any quenching step were $0.3672,0.4255,0.2015$, and 0.0059 while the expected proportions were $0.372,0.423,0.201$, and 0.004 , respectively.

${ }^{2}$ The mentioned proportions for realization 12 for the same method were $0.3730,0.4185,0.2024$, and 0.0060 while they were $0.3618297,0.4317658,0.2028174$, and 0.003587139 , respectively for realization 20. Clearly, both the realizations 16 , and 12 represent closer proportions to those of the target ones for soil classes 1, 2 and even 3 .
} 
(b) More reliable statistics in bigger classes: It is expected that the soil classes with the highest observed proportions, deduced from the borehole samples (considering necessary corrections for to the grid-transformation), would have the most reliable estimation of their proportions compared to the smaller classes. This is because of two reasons. First, the more dominant classes (based on the borehole data) have larger sample numbers and hence more reliabilities in their statistical inferences. The second basis could be that they also cover expectedly larger geometric sizes compared to the average spacing of the borehole samples, especially in the horizontal direction. Since the less observed proportions and hence the less coincidence of the boreholes with the smaller classes is due to their smaller geometric sizes compared to the average sample spacing, and vice versa. Therefore, the proportions of the small classes are prone to be approximated more inaccurately while the approximated proportions would be more reliable for the bigger classes. Hence, putting more emphasize on the reproduction of the proportions of the bigger soil classes is a sensible decision. In addition, in a volumetric view, if the proportions of the largest classes could be reproduced better, the volumes of the other classes also would not depart too much from their expected total volumes. This is because the total volume of the all categories is a fix amount for each of the realizations. However, if the volumetric deviations of the smaller classes are evaluated with a ratio rather than their volumes (similar to what is done in a chisquare test or by deviation rates), the deviations may seem very considerable. For example, because the expected volume of the soil category 4 , is 0.004 according to the categories proportions inferred from the borehole data considering the gridtransformation corrections, while the simulated volumetric proportion of the category 4 is 0.006 in a realization, there will be about $50 \%$ deviation of the proportion of this soil category from the expected value whereas the absolute volumetric difference is not so high. Besides, it is more probable that the approximated proportion of the category 4 (from the input data) is not that accurate and reliable due to the very low number of available samples from this class. The horizontal geometric sizes of this category should also obviously be much less than the boreholes spacing. However, for more frequent classes (in the boreholes samples), less variations should be acceptable, since their inferred proportions from the input data should expectedly be more precise. As a result, the suggested stepwise proportions deviation rate framework, that combines two insights of the 
overall and fractional volumetric deviations from the expectations and puts more merit on the most anticipated classes, seems a more proper solution for the proportions-reproduction evaluation problem. In fact, this scheme forces the absolute volumes of the soil classes to be reproduced much closer to those of target and therefore to the real ones. In addition, the deviation rate is more straightforward in representing the extent of the deviation from the expected proportions.

In this insight, as cited above, the most important and reliable soil-classes for the evaluation of the proportions reproduction are the classes 2, and 1 for the SISIM method, and classes 2, 1, and finally 3 for the TSIM method(s). The reason is that, these classes have the highest expected proportions and frequencies. Hence, the realizations that their proportions deviation rates of classes 2, and 1 (as well as class 3 in the TSIM method) from their expected proportions were less than $0.25 \%$ for the TSIM methods (except for the TSIM with two post-quenching-step method where the acceptable rate of $0.5 \%$ was considered), and less than $10 \%$ for SISIM method, were adopted as the acceptable realizations of these methods, in terms of the proportion- reproduction. As pointed out above, for the TSIM method with two post-quenching steps, the maximum acceptable deviation rate of $0.5 \%$ was considered because the deviations were, in general, higher for the results of this method compared to those of the TSIM methods with other numbers of quenching steps (i.e. no-, 1-, 3-, and 4-post-quenching steps). The maximum acceptable deviation rates for each simulation method were chosen so that the two or three best proportions-reproducing realizations could be differentiated. The reason for considering a lower acceptable value for maximum deviation rates in the TSIM with two-postquenching-step method is that, none of the realizations in this method have the deviation rate of the $0.25 \%$.

Table 5.2 summarizes and compares the results of the selected realizations produced by different simulation methods. The selection and comparison of the realizations in terms of proportions-reproduction was based on the two different mentioned methods of deviation rates (defined and proposed by the author of this dissertation) and chi-square test of homogeneity.

It should be clarified here that the proposed statistic which has been called the "sum of the rates of squared deviations" (SRSD) of the proportions here, was defined as following:

$$
\left.S R S D=\sum_{i=1}^{k} \mathbf{P}\left(O_{i}\right)-P\left(E_{i}\right)^{2}\right] / P\left(E_{i}\right)
$$


in which $P\left(O_{i}\right)$ is the observed proportion of the category i, and $P\left(E_{i}\right)$ is the expected proportion of the category i. Squaring the term $P\left(O_{i}\right)-P\left(E_{i}\right)$, makes the observed and expected proportions value positive and intensifies this difference while dividing the squared difference generates a rational value that compares this intensified positive difference to the expected proportions. SRSD resembles a chi-square statistic in some ways. Hence, SRSD facilitates the differentiation of better proportions-reproduction more clearly with a simpler calculation scheme compared to that of the chi-square test of homogeneity.

\section{b. Proportions-reproduction check using the chi-square test of homogeneity:}

Since, the real proportions of the soil categories are not exactly known and their inferred proportions form the boreholes are not necessarily accurate approximations of the real proportions, the consideration of the proportions inferred from the input data may not necessarily be accurate-enough in proportions-reproduction evaluations. Thus, the application of the chi-square test of homogeneity was suggested here as an alternative solution.

The chi-square statistic, in general, is defined as following:

$$
\chi^{2}=\sum_{i=1}^{k}\left(O_{i}-E_{i}\right)^{2} / E_{i}
$$

where the $\chi^{2}$ is the chi-square (or chi-squared) goodness of fit statistic, $O_{i}$ is the observed frequency (counts) for the bin (category) $i, E_{i}$ is the expected frequency for the bin $i$ (i.e. the reference frequency for the bin $i$ or let say the expected count for category $i$ ), and $k$ is the existing number of categories (McKillup and Darby Dyar 2010, pp. 234-236).

The problem of chi-square goodness of fit test here was that the sample sizes of input and simulation data were dramatically different. For that reason, another method had to be opted with the capability of comparing the proportions of the categories rather than their frequencies (counts).

It is a common question in earth sciences that the proportions of the considered categories in two or more groups of samples come from the same population or not? In contrary to the chi-square goodness of fit test, there are no expected frequencies or proportions of the categories in such problems. However, the chi-square test of homogeneity examines if the proportions of the categories in the two sample groups, i.e. the proportions observed in the boreholes versus the proportions calculated in the simulations, are heterogeneous that 
means they come from two different populations or they are homogenous and come the same population. The observed frequencies (counts) can be calculated simply by multiplying the observed proportions by the total number of their corresponding sample numbers (number of simulation nodes). The expected frequencies also can be calculated by a similar method, i.e. multiplication of the expected proportions by total number of data samples. It should be reminded again that, in the calculation of the proportions and the frequencies of the categories in simulations, corrections should be implemented due to the application of a transformed space.

The chi-square statistic for the test of homogeneity can be defined as following (Online source: "Chi-Square Test of Homogeneity." Chi-Square Test of Homogeneity, AP Statistics Tutorial. StatTrek.com¹; McKillup and Darby Dyar 2010, pp. 234-236):

$$
X^{2}=\sum_{c=1}^{N_{c}} \sum_{r=1}^{N_{r}}\left(O_{r, c}-E_{r, c}\right)^{2} / E_{r, c}
$$

where $X^{2}$ is the calculated chi-square statistic ${ }^{2}, \mathrm{~N}_{\mathrm{r}}$ is the number of data rows that indicates the number of sample groups (here $\mathrm{N}_{\mathrm{r}}=2$, i.e. two sample groups of ; the boreholes data, and simulation result), $\mathrm{N}_{\mathrm{c}}=k=4$ is the number of categories, $O_{r, c}$ is the observed frequency of data in row $r$, and column $c$ (in the contingency table), which is the data frequency of sample group $r$ (e.g. in borehole data; $r=1$, and in a simulation result; $r=2$ ) for category $c$ (i.e. soil classes $1,2,3$, and $4 ; c=1,2,3,4)$, the $E_{r, c}$ is the expected frequency of data in row $r$, and column $c$, or the data frequency of sample group $r$ for category $c$. The row and column here, respectively, stand for the row and column of a contingency table (McKillup and Darby Dyar 2010, pp. 234-236). Using the $X^{2}$ symbol instead of $\chi^{2}$ is because $X^{2}$ is a calculated or estimated value for chi-square statistic.

The expected frequency counts are computed separately for each population at each level of the categorical variable, according to the following formula:

$$
E_{r, c}=\frac{n_{r} \cdot n_{c}}{n}
$$

\footnotetext{
1 "Chi-Square Test of Homogeneity." Chi-Square Test of Homogeneity, AP Statistics Tutorial. StatTrek.com, Stat Trek Website. Web. Summer 2011. <http://stattrek.com/ap-statistics4/homogeneity.aspx>.
} 
where $E_{r, c}$ is the expected frequency count for the population $r$ at level $c$ of the categorical variable, $n_{r}$ is the total number of observations from the population $r, n_{c}$ is the total number of observations at sample group $c$, and finally $n$ is the total sample size (Online source: "Chi-Square Test of Homogeneity." Chi-Square Test of Homogeneity, AP Statistics Tutorial. StatTrek.com ${ }^{1}$ ).

Therefore, the null hypothesis $\left(\mathrm{H}_{0}\right)$ states that the proportions of all of the categories in a realization and those of the boreholes have come from the same population with the significance level of $\alpha$ whereas the alternative hypothesis (HA) says the contrary at least for one of the categories.

The null hypotheses will be rejected if the calculated chi-square $\left(X^{2}\right)$ is greater than the critical chi-square values function $\left(\chi_{(\alpha, D F)}^{2}\right)$ or the calculated p-value is less than the significance level of $\alpha$, for at least one of the categories:

$$
\begin{aligned}
& X^{2}>\chi_{(\alpha, D F)}^{2} \\
& p-\text { value }<\alpha
\end{aligned}
$$

where $\chi_{(\alpha, D F)}^{2}$ stands for the chi-square critical value function with the DF, degrees of freedom and a significance level of $\alpha$ (Online source: "Chi-Square Test of Homogeneity." Chi-Square Test of Homogeneity, AP Statistics Tutorial. StatTrek.com²).

This critical value can be derived from the standard chi-square distribution tables or chisquare calculators having the significance level $(\alpha)$ and the degrees-of-freedom (DF). The degrees of freedom for homogeneity of proportions test is calculated as following:

$$
\mathrm{DF}=\left(\mathrm{N}_{\mathrm{r}}-1\right)\left(\mathrm{N}_{\mathrm{c}}-1\right)
$$

where $\mathrm{N}_{\mathrm{r}}$, and $\mathrm{N}_{\mathrm{c}}$ are the numbers of columns, and rows in the contingency table, respectively. In other words, as an example in this study, $\mathrm{N}_{\mathrm{r}}=2$ is the number of sample groups that should be evaluated for being homogenous with one another (i.e. derived from

\footnotetext{
1 "Chi-Square Test of Homogeneity." Chi-Square Test of Homogeneity, AP Statistics Tutorial. StatTrek.com, Stat Trek Website. Web. Summer 2011. <http://stattrek.com/ap-statistics4/homogeneity.aspx>.

2 "Chi-Square Test of Homogeneity." Chi-Square Test of Homogeneity, AP Statistics Tutorial. StatTrek.com, Stat Trek Website. Web. Summer 2011. <http://stattrek.com/ap-statistics4/homogeneity.aspx>.
} 
the same population), and $\mathrm{N}_{\mathrm{c}}=4$ is the number of columns in this table which is the number of existing categories. Therefore, the degrees-of-freedom for the present study is:

$$
\mathrm{DF}=\left(\mathrm{N}_{\mathrm{r}}-1\right)\left(\mathrm{N}_{\mathrm{c}}-1\right)=(2-1)(4-1)=1 \times 3=3
$$

In this case, $k$ is the number of soil classes. It is clear from the definition of $\chi^{2}$ that the smaller the $X^{2}$ (estimated $\chi^{2}$ ), the closer the evaluated simulation proportions to those of the reference will be ("Chi-Square Test of Homogeneity." Chi-Square Test of Homogeneity, AP Statistics Tutorial. StatTrek.com).

\section{(3) The proportions-reproduction checks in the test site:}

The distributions were calculated and checked for the different realizations of the SISIM, and TSIM (transition-probability Markov chain simulation) methods including the TSIM simulation without quenching steps, as well as the TSIM simulations with one-, two-, three-, and four-step post-quenching phases. The proportions of the categories in each realization of the various simulation method were obtained from their corresponding debugging (*.dbg), and the generic "tsim.out" files. To avoid missing the produced results in the "tsim.out" files, in the TSIM method(s), the "tsim.out" files of the TSIM program from T-PROGS software were renamed, prior to running any new simulation. For example, here, they were renamed to the files with the prefixes names, the same as the output realizations file names, and the suffixes of "_out.out".

In the" post-quenching" steps, the simulated annealing is conducted on the previously generated realizations of various simulation methods. The post-quenching steps were conducted to reduce the differences between the transition-probabilities of a simulation and the target Markov chain models of them. Thus, the "post-quenching step" term was applied here for the further quenching steps performed on the available realizations from the previous stages. The mentioned available realizations could be the product of the realizations with or without further quenching steps from the previous stages (mainly about the TSIM method). In fact, in each stage, only one quenching step was added to the previous realizations. Therefore, the TSIM simulations were started by conducting the TSIM simulation without any quenching steps, then a quenching step was performed on the realizations of the previous stage (i.e. on the TSIM method without any quenching step) to result the TSIM realizations with one quenching step, then another quenching 
step was performed on the result of the previous stage (i.e. on the TSIM with one "postquenching step") to achieve the TSIM realizations with two post-quenching steps, and so on.

Table 5.2 Comparison of selected realizations produced with different simulation methods for proportions reproduction using two different suggested histogram reproduction test methods

\begin{tabular}{|l|c|c|}
\hline $\begin{array}{c}\text { Realizations with acceptable } \\
\text { proportions-reproduction }\end{array}$ & $\begin{array}{c}\text { Based on deviation } \\
\text { rates } \\
\text { Simulation method } \\
\text { (maximum acceptable } \\
\text { deviation rate) }\end{array}$ & $\begin{array}{c}\text { Based on chi-square } \\
\text { test of homogeneity }\end{array}$ \\
\hline $\begin{array}{l}\text { SISIM (Sequential Indicator } \\
\text { Simulation method) }\end{array}$ & $\begin{array}{c}12,38,37 \\
(0.1)\end{array}$ & $37,12,65$ \\
\hline $\begin{array}{l}\text { TSIM without quenching (Markov } \\
\text { chain simulated annealing } \\
\text { optimization) steps. }\end{array}$ & $\begin{array}{c}16,12,1,20 \\
(0.025)\end{array}$ & $20,16,12,1$ \\
\hline $\begin{array}{l}\text { TSIM with 1-post-quenching (Markov } \\
\text { chain simulated annealing } \\
\text { optimization) steps. }\end{array}$ & $\begin{array}{c}19,12,18,4 \\
(0.025)\end{array}$ & $7,6,19,12$ \\
\hline $\begin{array}{l}\text { TSIM with 2-post-quenching (Markov } \\
\text { chain simulated annealing } \\
\text { optimization) steps. }\end{array}$ & $\begin{array}{c}12,3,16,9 \\
(0.025)\end{array}$ & $19,16,11,17$ \\
\hline $\begin{array}{l}\text { TSIM with 3-post-quenching (Markov } \\
\text { chain simulated annealing } \\
\text { optimization) steps. }\end{array}$ & $\begin{array}{c}9,4,12,1 \\
(0.025)\end{array}$ & $3,16,9,11$ \\
\hline $\begin{array}{l}\text { TSIM with 4-post-quenching (Markov } \\
\text { chain simulated annealing } \\
\text { optimization) steps. }\end{array}$ & $\begin{array}{c}19,18,16,17 \\
(0.025)\end{array}$ & $19,18,16,15$ \\
\hline
\end{tabular}

The reason for using the "post-quenching" technique was to save the simulation time by avoiding the repetitions of quenching stages. For example, to achieve a TSIM simulation with a three-step post-quenching, one can simply carry out a quenching step on the realizations of a TSIM simulation with two post-quenching steps, which was actually produced by performing a quenching step on the realizations of the one-step postquenched TSIM simulation, and so forth.

Table 5.2 summarizes the proportions reproduction test results of the geostatistical simulations. The table includes the accepted realizations for all applied simulation techniques based on the two stated proportions-reproduction test methods.

(4) The density of the simulation grids: 
In this study also, the effect of using coarser grids on the proportions reproduction was tested. As it has been expected, the results were dependent on the grid sizes so that each grid size lead to a different proportions-reproduction quality. Stepwise application of the different grid sizes is actually a part of the multiple-grid simulation strategy embedded in the GSLIB simulation programs to improve the simulation algorithm by taking the longrange structures into account. This strategy however, has not been included in the TSIM program of the TPROGS. The multiple-grid simulation concept is to perform the simulation in two or more stages; i.e. starting from the coarser grids to the finer ones. In other words, a coarser grid is simulated first. Then this coarse grid is used as the conditioning data for another finer-grid simulation and so on (Deutsch and Journel 1998, p. 190). One of the advantages of using the multiple-grid strategy is that it leads to a better reproduction of the long-distance variogram structures. Therefore, adding this strategy to the TSIM program of the TPROGS could also be suggested to enhance the efficiency of the TSIM algorithm for a better spatial structures reproduction.

\section{The number of realizations in different simulation methods:}

It should be reminded here that the number of realizations for the TP/MC (transitionprobability Markov chain) method was less (i.e. twenty realizations) than those of the SISIM method (i.e. one hundred realizations). There were two reasons for generating only twenty realizations in each of the TSIM simulation rounds:

(1) Technical limitations: The technical limitations, because of exceeding the capacity of the TSIM program for such a big and fine-resolution simulation grid $(\mathrm{nx}=175, \mathrm{ny}=124, \mathrm{nz}=28$; with unit cell size of $12.5 \mathrm{~m} \times 12.5 \mathrm{~m} \times 0.25 \mathrm{~m})$ from its limits after producing twenty realizations. Even by improving some capacity settings and parameters of the program, the TSIM program exceeded its limits. Perhaps, this capacity could still be enhanced. Due to the limited Ph.D. time, further enhancements were not followed up at this moment.

(2) Low variations in TSIM method results: A considerably lower variations was observed among the realizations of a TSIM method compared to those of the SISIM method, in terms of several statistical criterions such as proportions of the soil categories, variograms, and transition-probabilities of the realizations (see the proportions-reproduction tables as well as the TPs and indicator variograms- 
reproduction in this section). Then, adding more numbers of realizations would not add a significant merit to the analysis, even if the technical limitations would not be encountered. (Yet, a set of 20 realization of the SISIM method was produced and checked for their indicator variogram spectrum. As Observed in these results, they covered nearly the same variation spectrum of the variograms. However, due to much higher variations of SISIM compared with the TSIM method, I preferred to keep 100 realizations for the SISIM method (see Figure 6.1 and Figure 6.2).

\section{(5) More details about the chi-square test for homogeneity of proportions:}

As it has already been explained in this context, the chi-square statistic for the homogeneity of populations test was calculated for each of the realizations produced by various simulation methods.

In order to formulate the proportions reproduction of the realizations for each simulation method using the chi-square homogeneity of proportions test, the null and alternative hypotheses and the significance level (the critical p-value) should be defined. The significance level $(\alpha)$ is the probability of improperly rejecting the null hypothesis (H0) and in general, the null hypothesis is the hypothesis of "no difference" or "no effect" (McKillup, Steve, and Darby Dyar 2010, p. 13). In this problem, the null hypothesis is that the proportions of each soil category and those inferred from the borehole data (with necessary corrections explained in Section 2.1.6) reflect samples from the same population.

The critical p-value (or significance level of $\alpha$ ) for selecting the four and three best realizations, for TSIM, and SISIM methods, respectively, was considered as a criterion to evaluate the proportions reproduction qualities among different simulation methods. For instance, if for the simulation methods of $\mathrm{A}$ and $\mathrm{B}$, the significance levels for selecting the four best realizations are $\alpha_{A}$ and $\alpha_{B}$, respectively, $\alpha_{A}$ can be compared with $\alpha_{B}$ to analyze and decide which method have reproduced the target proportions better. In this example, if $\alpha_{A}>\alpha_{B}$, it can be concluded that the realizations produced by method A, could reproduce the real proportions better. In other words, the method A, worked better in terms of proportions reproduction in this example. 
To evaluate the simulations using the $X^{2}$ values for the proportions reproduction in this stage, the significance levels with which, in each simulation methods, the three best realizations in SISIM method and four best realizations in TSIM method(s) ${ }^{1}$ could be selected, were suggested. The reason for selecting a less number of realizations as the best proportions-reproducing ones in the SISIM method was that, this method had considerably lower proportions reproduction quality compared to that of the TSIM method and the quality of the realization would drop dramatically if a more number of realizations were selected. Lower critical p-values were selected for the simulation methods with a less quality of proportions reproduction (e.g. SISIM or TSIM without quenching step methods) and higher critical p-values were selected for the simulation methods with a better quality of proportions reproduction (e.g. TSIM methods with one or more numbers of quenching steps). It was concluded that, performing additional quenching steps, in general, improved the proportions reproduction quality of simulations. Although after the first quenching step, in the TSIM with two-post-quenching steps, the proportions-reproduction became even slightly worse. Then, it did not change significantly in three-step post-quenching stage. However, the proportions-reproduction was improved considerably in the four-step post-quenching phase. Moreover, in all the TSIM methods, the suggested critical p-values are considerably above the conventional significance level of 0.05 . Therefore, with the significance level of 0.05 , none of the selected realizations of the TSIM method could be rejected. Yet, a significance level of $0.15(15 \%)$ could be suggested for all of the selected realizations. Even in this significance level, all the accepted realizations (i.e. four best realizations in each TSIM method) fall in the statistical level of not being rejected for the TSIM methods.

Sorting the realizations in terms of their $X^{2}$ homogeneity statistics in an ascending order, the realizations with the least estimated chi-square values $\left(X^{2}\right)$ were located on the top of the list, representing the realizations which best reproduce the target proportions.

\section{(6) Summarizing the results of the chi-square test of homogeneity to evaluate the goodness of proportions-reproduction:}

Table 5.3 summarizes some descriptive statistics of the chi-square values for the homogeneity of proportions of the realizations (with the boreholes data), generated by

\footnotetext{
${ }^{1}$ When the term "TSIM methods" has been implemented in this context, the aim was to refer to the application of the TSIM method with different post-quenching steps.
} 
different simulation methods. The minimum, maximum, average, and the standard deviations of $X^{2}$ corresponding to each of the simulation methods are reflected in this table. In addition, the related p-values for each of the $X^{2}$ statistic together with the corresponding coefficient of variation of the standard deviation of $X^{2}$ have been placed in the parentheses.

The mentioned descriptive statistics were chosen to represent the statistical location and dispersion of the calculated chi-square $\left(X^{2}\right)$ values.

Table 5.3 Summary of chi-square statistics for different simulation methods applied in this study.

\begin{tabular}{|c|c|c|c|c|}
\hline Simulation method & $\begin{array}{c}X_{\min }^{2} \\
\text { (corresponding } \\
\left.P_{X_{\min }^{2}}\right)\end{array}$ & $\begin{array}{c}X_{\max }^{2} \\
\text { (corresponding } \\
\left.P_{X_{\max }^{2}}\right)\end{array}$ & $\begin{array}{c}X_{\text {average }}^{2} \\
\text { (corresponding } \\
P_{X_{\text {average }}^{2}} \text { ) }\end{array}$ & $\begin{array}{c}\text { Standard } \\
\text { deviation of } \\
X^{2} \\
\text { (corresponding } \\
\text { coefficient of } \\
\text { variation) }\end{array}$ \\
\hline $\begin{array}{l}\text { TSIM without quenching } \\
\text { (Markov chain simulated } \\
\text { annealing optimization) } \\
\text { steps. }\end{array}$ & $\begin{array}{l}0.888135 \\
(0.8282)\end{array}$ & $\begin{array}{c}33.35429 \\
(2.7112 \mathrm{e}-7)\end{array}$ & $\begin{array}{l}8.584887566 \\
(0.0353)\end{array}$ & $\begin{array}{l}8.124094 \\
(0.946325)\end{array}$ \\
\hline $\begin{array}{l}\text { TSIM with 1-post-quenching } \\
\text { (Markov chain simulated } \\
\text { annealing optimization) } \\
\text { steps. }\end{array}$ & $\begin{array}{l}0.018947599 \\
(0.9993)\end{array}$ & $\begin{array}{l}0.79631175 \\
(0.8503)\end{array}$ & $\begin{array}{l}0.313639749 \\
(0.9574)\end{array}$ & $\begin{array}{l}0.245226 \\
(0.781872)\end{array}$ \\
\hline $\begin{array}{l}\text { TSIM with 2-post-quenching } \\
\text { (Markov chain simulated } \\
\text { annealing optimization) } \\
\text { steps. }\end{array}$ & $\begin{array}{l}0.189506385 \\
(0.9792)\end{array}$ & $\begin{array}{l}0.896819433 \\
(0.8261)\end{array}$ & $\begin{array}{l}0.428922857 \\
(0.9342)\end{array}$ & $\begin{array}{l}0.217569 \\
(0.507245)\end{array}$ \\
\hline $\begin{array}{l}\text { TSIM with 3-post-quenching } \\
\text { (Markov chain simulated } \\
\text { annealing optimization) } \\
\text { steps. }\end{array}$ & $\begin{array}{l}0.344708282 \\
(0.9514)\end{array}$ & $\begin{array}{l}0.693566396 \\
(0.8747)\end{array}$ & $\begin{array}{l}0.500907457 \\
(0.9186)\end{array}$ & $\begin{array}{l}0.091308 \\
(0.182285)\end{array}$ \\
\hline $\begin{array}{l}\text { TSIM with 4-post-quenching } \\
\text { (Markov chain simulated } \\
\text { annealing optimization) } \\
\text { steps. }\end{array}$ & $\begin{array}{l}0.000510806 \\
(0.9999)\end{array}$ & $\begin{array}{l}0.840421978 \\
(0.8397)\end{array}$ & $\begin{array}{l}0.20874874 \\
(0.9761)\end{array}$ & $\begin{array}{c}\mathbf{0 . 2 8 1 9 1 8} \\
(1.350514)\end{array}$ \\
\hline $\begin{array}{l}\text { SISIM (sequential indicator } \\
\text { simulation method) }\end{array}$ & $\begin{array}{l}8.51003149 \\
(0.0365)\end{array}$ & $\begin{array}{l}138.6058825 \\
(0)\end{array}$ & $\begin{array}{l}48.8278298 \\
(0)\end{array}$ & $\begin{array}{l}22.84308 \\
(0.467829)\end{array}$ \\
\hline
\end{tabular}

These results can be summarized and interpreted as following: 
(a) In general, the TSIM realizations reproduce the soil categories distribution (i.e. the proportions) much better than those of the SISIM method. In the SISIM method, the average of the calculated chi-squares is $X_{\text {average }}^{2}=48.82783$. The $X^{2}$ ranges from 8.5100 to 138.6059 for different realizations whereas the $X^{2}$ has the overall average of 2.0074, and ranges from 0.0005 to 33.3543 for the TSIM method. The decision that which simulation could reproduce the proportions better was based on the fact that the lower the $X^{2}$, the better the proportions-reproduction.

(b) In all of each TSIM methods, the majority of realizations (for example, 12 realizations out of 20 in the TSIM without-quenching-step method) reproduce the proportions adequately with the significance level of 5\%. In addition, all of the realizations of the TSIM method(s) with quenching steps reproduced the soil proportions with the significance level of $80 \%$ or better and most of the realizations (at least 12 out of 20 realizations) reproduced the proportions with the significance level of at least $90 \%$. Therefore, it can be concluded that the quality of proportions-reproduction was notably higher in all of the TSIM methods especially in the TSIM method with quenching steps.

(c) Only three realizations of the SISIM method fell in an acceptable range with the significance level of $1 \%$. The best realization of the SISIM method (i.e. the realization number 37), reproduced the proportions at the significance level of $3.65 \%$ which is just under the usual acceptance significance level of the chi-square tests $(5 \%)$. Hence, the proportions-reproduction quality in the SISIM method was generally poor especially compared to that of the TSIM methods.

(d) Adding the quenching (or post-quenching) steps to the TSIM method increased the proportions-reproductions significantly. However, the quality of the proportions-reproductions did not increase regularly with the increasing of the numbers of quenching steps.

(e) Paying attention to the TSIM with four quenching steps, nearly all of the generated realizations (17 out of 20 realizations) reproduced the proportions with a $90 \%$ significance level. Thus, the proportions-reproduction was the best in TSIM 
simulation with four quenching steps, based on the chi-square test of homogeneity. In addition, the selected realizations from both the chi-square homogeneity and the deviation rates test (proposed by the author of this dissertation here) were more common for the TSIM with four-post-quenching steps. A lot of common selected realizations between two methods of deviation rates and chi-square test of homogeneity while with a less computational costs and complexities for the former bedsides the best accuracy of the realizations in this simulation method (based on the proportions-reproduction tests), suggested that the deviation rates framework could be efficiently applied in the similar problems for proportions-reproduction checks. More details about this method and its analogues statistical method has been addressed in part (2)a, section 5.3.3 from Chapter 5. Perhaps, some conditions should be taken into consideration before applying the proportions deviation method such as the enough number of input sample data. It seems that inferring an accurate-enough representative histogram from the input data is the key point in the application of the deviation rates method.

Regarding the fact that the two proposed proportions-reproduction tests, here, suggested different but with many common and similar selected realizations, it can be concluded that the two methods lead to the similar results. The two methods of the proportions "deviation rate" and the chi-square test of homogeneity for evaluating the proportionsreproduction of the realizations are suggested to be applied to the similar cases where the categorical variables are being simulated. In addition, the proportions deviation rate scheme with a simpler framework can be applied in the similar problems leading to more or less similar results.

Table 5.4 illustrates the two methods of the proportions reproduction test ("proportions deviation rates" and the chi- test of homogeneity) for the realization number 37 of the SISIM method as an example. The "Global proportion" column is the proportions calculated from the boreholes considering the necessary grid-transformation corrections, the "Calculated proportions" come from the calculation of the proportions of the realization number 37 of the SISIM method obtained from the simulation debugging file, and the next columns show the relevant calculations. 
Table 5.4 Illustration of the calculations frameworks of the chi-square test of the homogeneity of proportions, and deviation-rate, for the realization number 37 of the SISIM geostatistical simulation method.

\begin{tabular}{|c|c|c|c|c|c|c|c|}
\hline $\begin{array}{l}\text { Soil } \\
\text { class }\end{array}$ & $\begin{array}{c}\text { Global } \\
\text { proportion }\end{array}$ & $\begin{array}{l}\text { Calculated } \\
\text { proportion }\end{array}$ & $\begin{array}{c}\text { Element } \\
X^{2} \\
\text { realizations }\end{array}$ & $\begin{array}{c}\text { Element } \\
X^{2} \\
\text { boreholes }\end{array}$ & $\begin{array}{l}\text { Total } \\
X^{2}\end{array}$ & $\begin{array}{l}\text { Deviation } \\
\text { rate }\end{array}$ & $\begin{array}{c}\text { Was the } \\
\text { deviation } \\
\text { rate }<=0.1 \\
?\end{array}$ \\
\hline $\mathrm{Cl} 1$ & 0.372 & 0.3486 & 0.007193 & 2.5877 & \multirow{4}{*}{8.5100} & 0.0629 & 1 \\
\hline $\mathrm{Cl} 2$ & 0.423 & 0.4189 & 0.000165 & 0.0593 & & 0.0097 & 1 \\
\hline $\mathrm{Cl} 3$ & 0.201 & 0.2258 & 0.012846 & 4.6211 & & 0.1234 & 0 \\
\hline $\mathrm{Cl} 4$ & 0.004 & 0.0061 & 0.003387 & 1.2183 & & 0.5250 & 0 \\
\hline
\end{tabular}

\subsubsection{Transition-probabilities-reproduction:}

The transition-probabilities-reproduction of the generated simulations by different simulation methods reflects how close or similar the transition-probabilities (TPs) of the simulations to their corresponding MC models were. This test was implemented as a spatial variability index to verify one of the minimum acceptance criteria for geostatistical simulations. As discussed before, variograms were the other spatial variability tools applied in this study to asses the quality of generated realizations as one of the minimum criteria for accepting each realization. The variograms-reproduction of the realizations has been discussed in the coming section. In this section, the focus is on the evaluation of the transition-probabilities as another spatial variability tool to assess the spatial-variabilityreproduction of realizations. The author proposes here, to consider the transitionprobabilities reproduction as another minimum acceptance criterion of any realization in similar problems since the transition-probabilities capture the spatial variability structure of the categories, in terms of the change of state of each soil or facies category to any (other or the same) state. Therefore, the transition-probabilities of the simulations should reflect some spatial variability features better than what variograms can. It has been proposed here to apply the both variograms and transition-probabilities to assess the spatial-variability-reproductions of the generated realizations. 
Regarding the fact that there were lots of realizations from different geostatistical simulation methods and different simulation parameters (such as the number of quenching steps in the TSIM method), it would practically be extremely tedious to compare the transition-probabilities of all the simulation results one-by-one (e.g. by plotting them in a graph or performing an analytical comparison) with their corresponding Markov chain models. The technical limitations regarding the software also were required to be considered. To overcome these problems, two strategies could be considered:

(a) To assess the acceptability of each realization separately, the realizations which reproduced the soil proportions the best were selected at the first step. Then, these selected realizations were suggested to be checked for their transitionprobabilities- reproduction (abbreviated here as TPs-reproduction) in the next step. One of the good reasons to evaluate the proportions-reproduction initially was that, a realization with closer proportions to those of the model would be expected to show closer variogram and transition-probability sills to those of the model. The reason for this prospect is that, the expected sill of an indicator variogram $(c=p(1-p))$ or a TP is a function of the proportion of that soil category (i.e. $p_{k}$ ). It is worth reminding again that, the sill of $t_{m k}(h)$ is tangent to the $p_{k}$ (proportion of category $k$ ) line at the infinity, whether $m=k$ or $\mathrm{m} \neq$ k. Therefore, an acceptable proportions-reproduction can, at least, ensure a better sills-reproduction for variograms and TPs. It was shown before about the SISIM method that, the realizations with poorer proportions-reproduction demonstrated, in general, a weaker variograms-reproduction while the realizations with a better proportions-reproduction, represented commonly, a better variograms-reproduction as well. Hence, it can be expected that, the realizations which reproduced the target proportions better, should also be capable of better reproducing the spatial variability including the TPs. Figure 5.7 to Figure 5.16 represent the TPs of the best selected realizations (in terms of their proportions-reproductions)from TSIM method versus their corresponding TP Markov chain models. Therefore, a better TPs-reproduction was also expected in addition to a better variograms-reproduction, of the selected realizations. The TPs-reproduction of the selected realizations seems acceptable and better for the simulations with some quenching steps. Obviously, it should be considered that the effect of a better proportions-reproduction on the better 
TPs-reproduction should be in a specific extent. Because, if the general image of the patterns become similar, the proportions also would be similar. However, in general, a better proportions-reproduction can not completely guarantee a better variograms- or TPs-reproduction. Ideally, a sequential geostatistical simulation should reproduce the spatial variability structure (variograms and TPs) of the simulation model. Yet, in practice, the outcome of the sequential geostatistical simulations can not really reproduce the variograms or TPs because of the limitations embedded in the algorithms. The most important factor which affects the variograms/TPs reproduction is the long-range variations (Zanon and Leuangthong 2005). According to Zanon and Leuangthong (2005), having a more abundant data-set and using the multiple-grid search strategy can improve the long-range-variation-reproduction of the sequential simulations. Nevertheless, in general, choosing the best proportions-reproducing realizations can narrow the range of realizations which should be evaluated for its variograms-/TPs-reproduction efficiency by suggesting the more acceptable realizations.

(b) To assess the overall efficiency and quality of all the realizations of a simulation method, one could also calculate the average transition-probabilities of all realizations of a geostatistical simulation method and plot them together with their corresponding Markov chain models in one plot and compare them with each other among different methods. Similarly, the average indicator variograms of all realizations of a simulation method could also be calculated and plotted together with their relevant indicator variogram models, to evaluate the overall efficiency of a simulation method for its variograms-reproduction. These evaluations were initially attempted by the author using a special trick (i.e. constructing a big three-dimensional dummy model made by all the realizations of each simulation method which includes the grids of all of the realizations while the spatial continuity measures of the new model have the same value as that of the overall average of all realizations). However, this procedure was not accomplished further because the evaluations using the first method and plotting of the variograms and TPs of all realizations of each method in a graph seemed sufficient and even more efficient. Hence, only the results of first method have been brought and discussed here. 
As mentioned above, the transition-probabilities of the best realizations, chosen in terms of their proportions-reproduction, and their corresponding Markov chain models were plotted at the same graphs and compared with each other to evaluate the transitionprobabilities reproduction. The selection of the best realizations, in terms of the proportions-reproduction was a compromising decision between the results of two different proportions-reproduction-test methods of the chi-square test of homogeneity, and the deviation rates frameworks.

As Figure 5.7 to Figure 5.16 represent, the TPs of the selected realizations match their relevant Markov chain models, in general, more or less well. This match was better for the TSIM methods, especially for the TSIM simulations with some quenching steps. However, the match between the TPs of the realizations and those of the models were weaker in vertical direction, especially for the class 4 which had the least observations frequency. These interpretations had been obtained by a graphical comparison of the transition-probabilities of the realizations and models. It has been explained in the coming section that, there are some analytical frameworks suggested in a number of literature to evaluate the variograms and other statistics-reproduction for simulation evaluations by analytical methods. However, it has been shown there, why the application of these measures was not suitable in this case so that the graphical method was preferred. Further attempts can be suggested to develop and suit similar analytical criterions to evaluate the generated realizations. 


\section{Geotechnical classes Goettingen soils $\mathrm{Hz}$. Transition Probabilities}

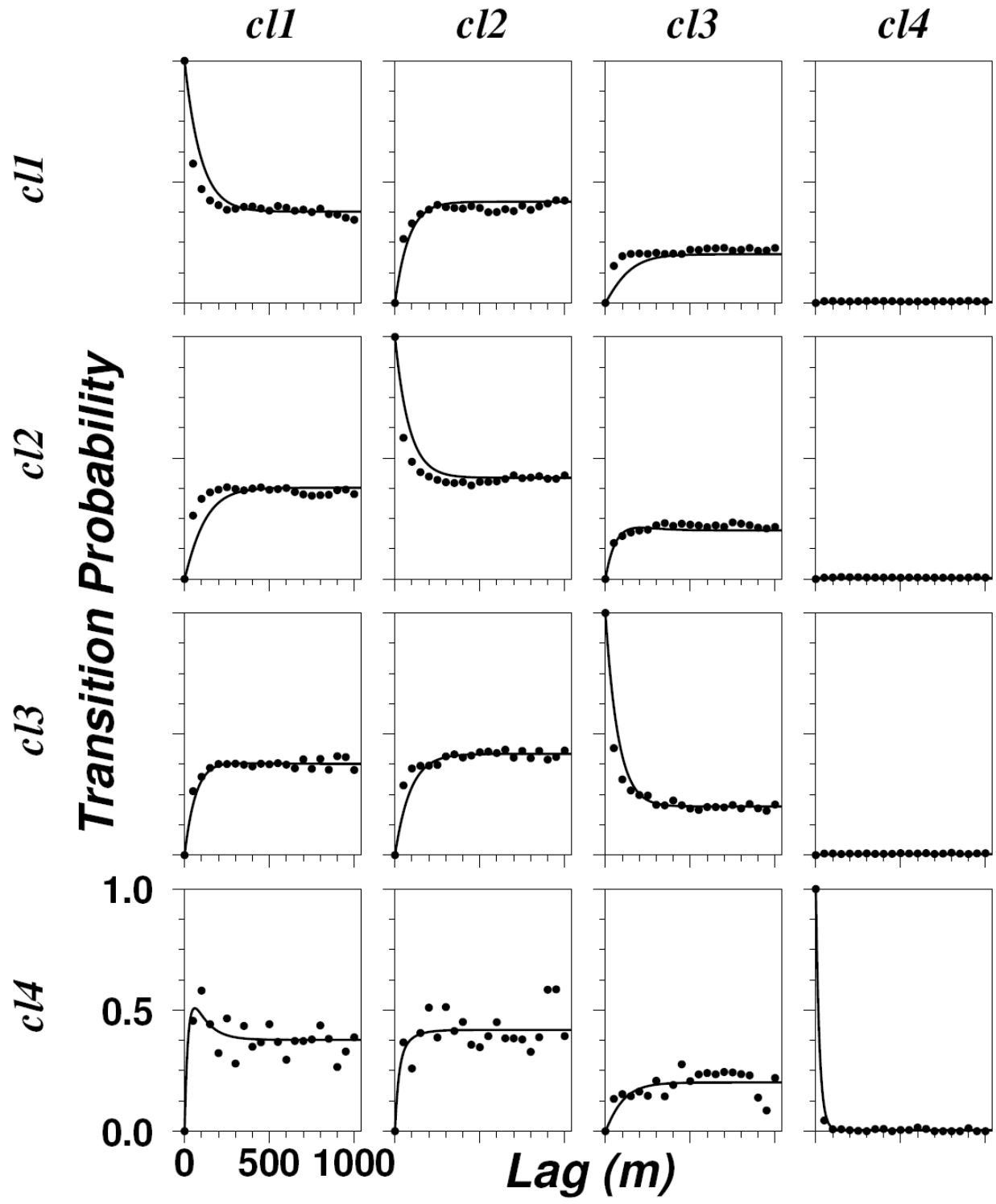

TSIM_nq_r16

MC model

Figure 5.7 Horizontal transition-probabilities among the soil classes for realization 16 generated by TSIM without any quenching step method (dots) and corresponding Markov chain models (solid lines). 


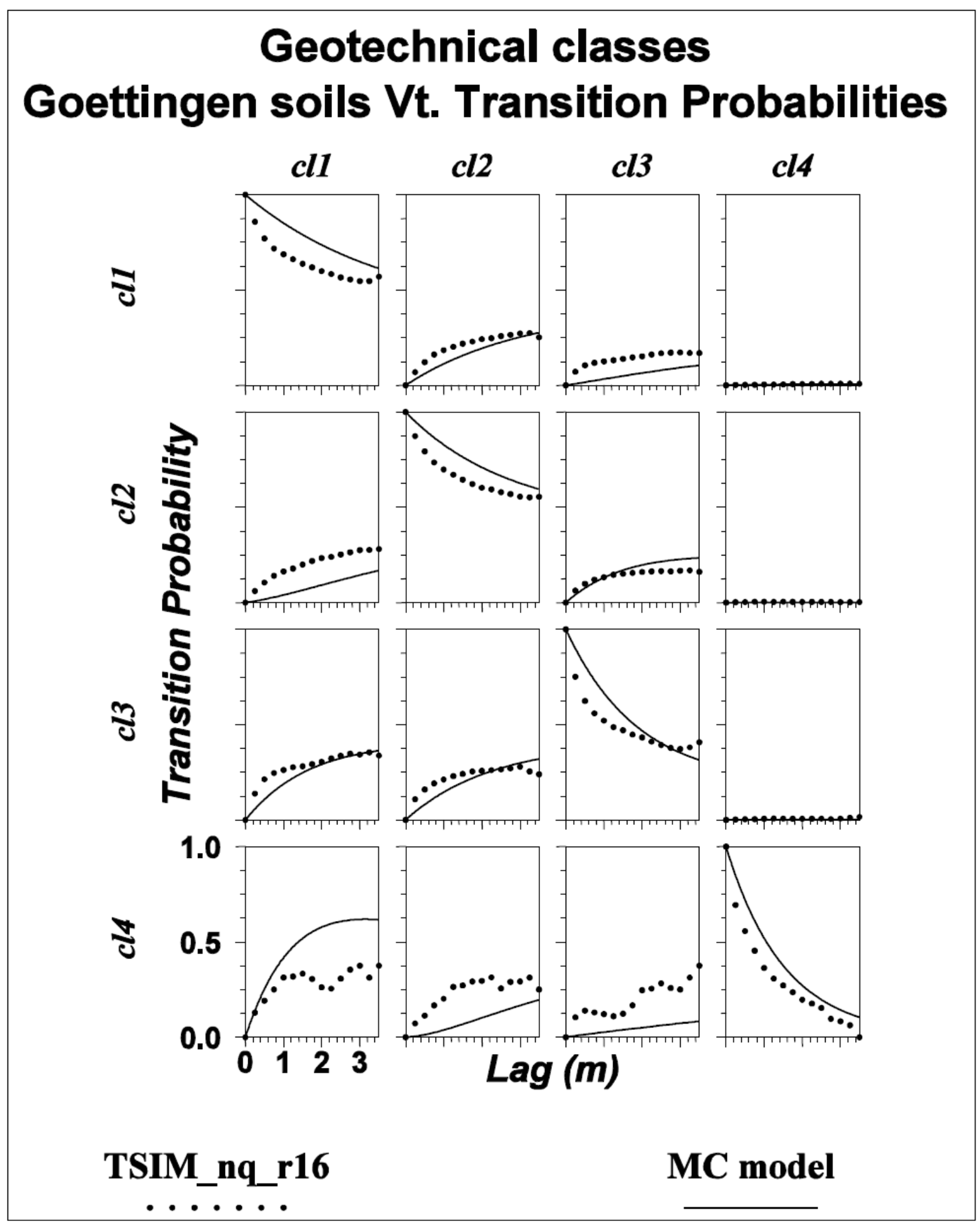

Figure 5.8 Vertical transition-probabilities among the soil classes for realization 16 generated by TSIM without any quenching step method (dots) and corresponding Markov chain models (solid lines). 


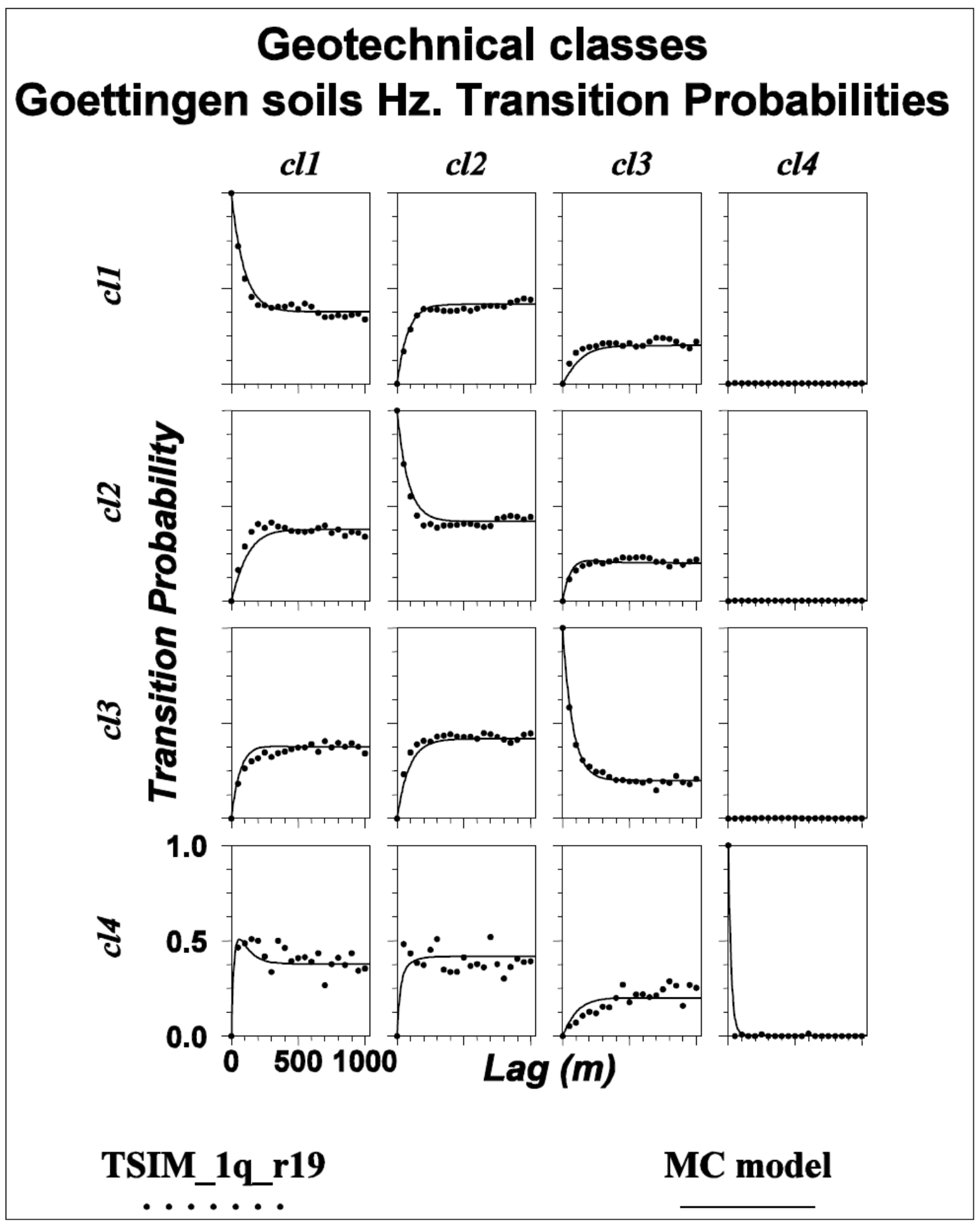

Figure 5.9 Horizontal transition-probabilities among the soil classes for realization 19 generated by TSIM method with a one-step post-quenching phase (dots) and corresponding Markov chain models (solid lines). 


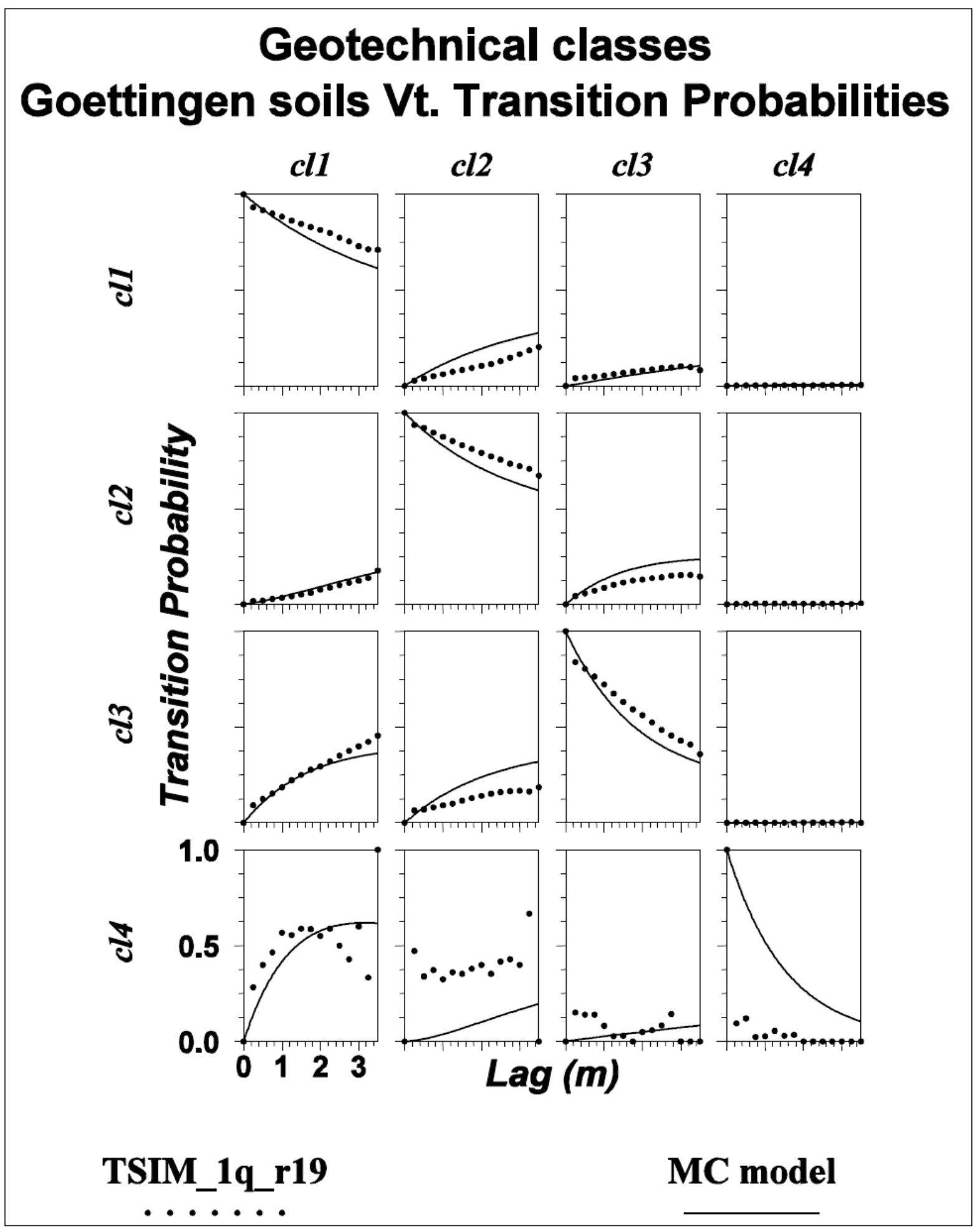

Figure 5.10 Vertical transition-probabilities among the soil classes for realization 19 generated by TSIM method with a one-step post-quenching phase (dots) and corresponding Markov chain models (solid lines). 


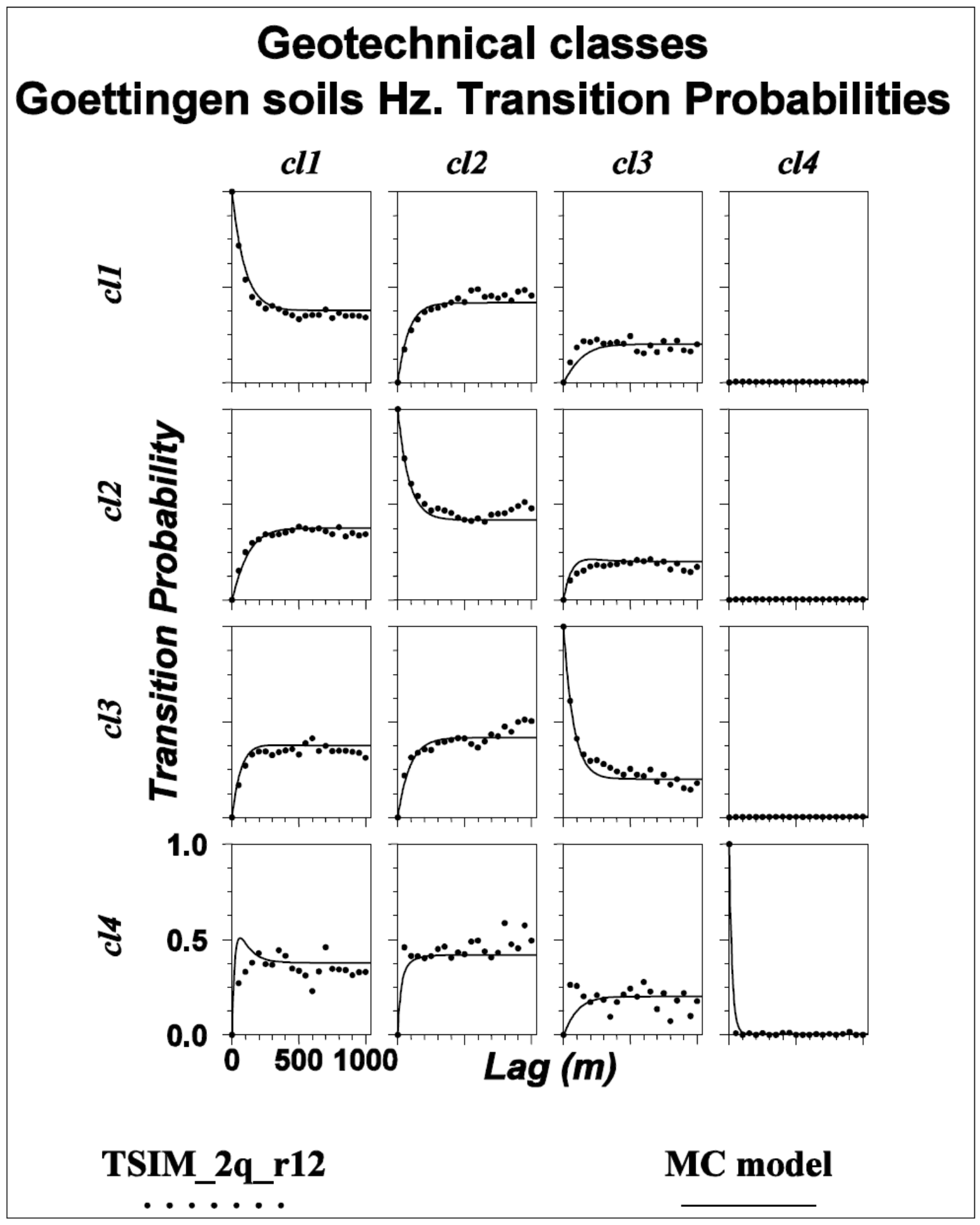

Figure 5.11 Horizontal transition-probabilities among the soil classes for realization 12 generated by TSIM method with a two-step post-quenching phase (dots) and corresponding Markov chain models (solid lines). 


\section{Geotechnical classes Goettingen soils Vt. Transition Probabilities}
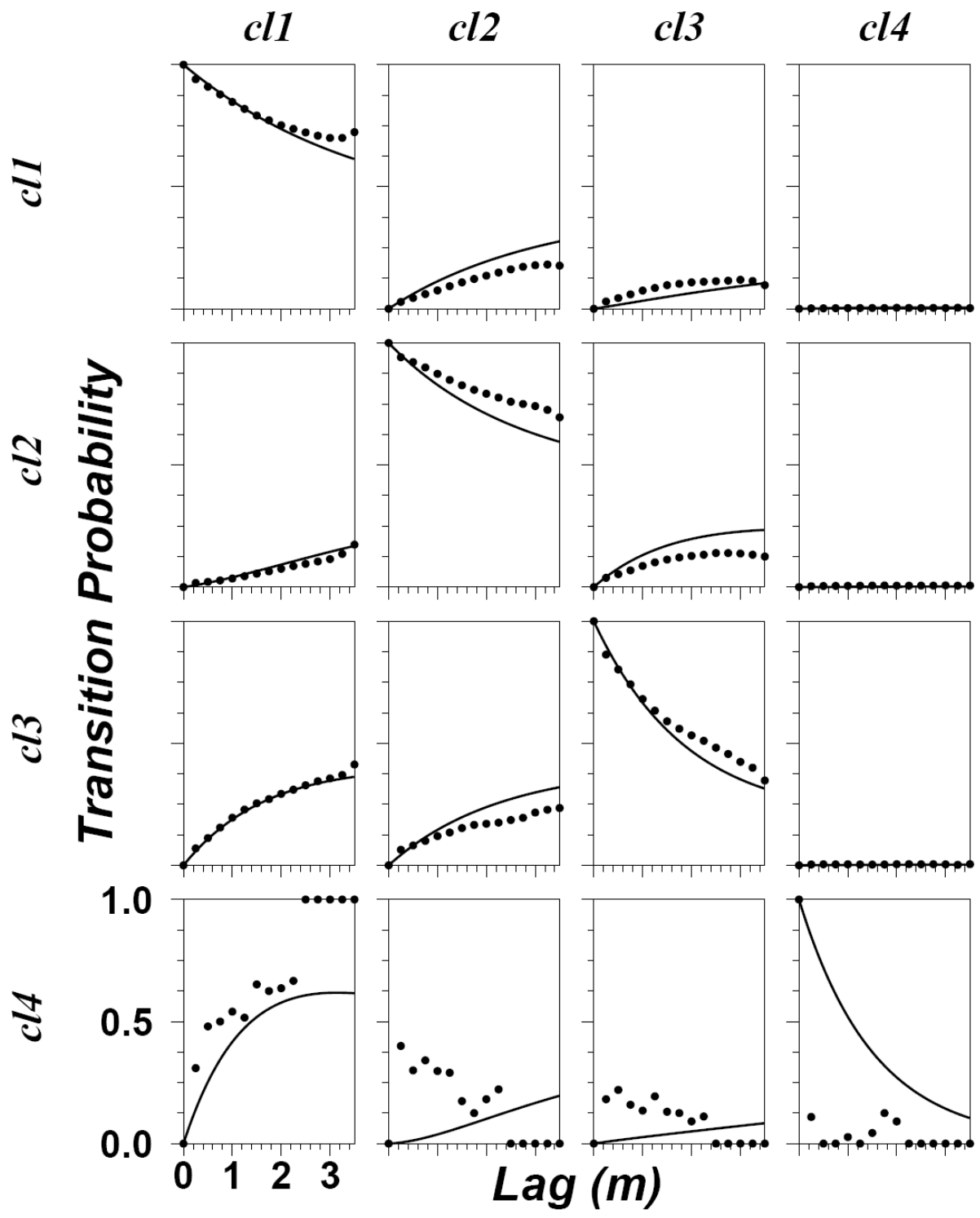

TSIM_2pq_r12

Figure 5.12 Vertical transition-probabilities among the soil classes for realization 12 generated by TSIM method with a two-step post-quenching phase (dots) and corresponding Markov chain models (solid lines). 


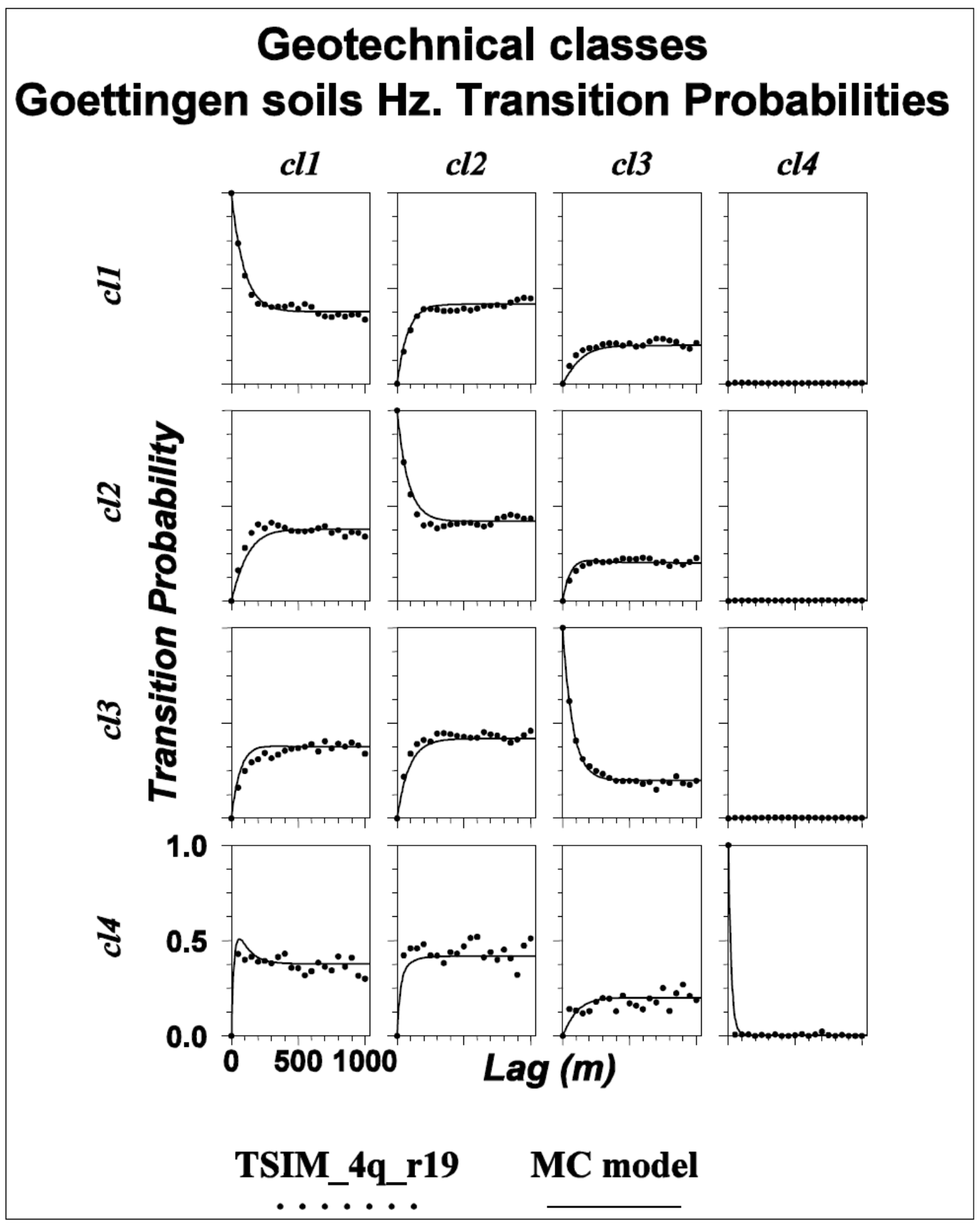

Figure 5.13 Horizontal transition-probabilities among the soil classes for realization 19 generated by TSIM method with a four-step post-quenching phase (dots) and corresponding Markov chain models (solid lines). 


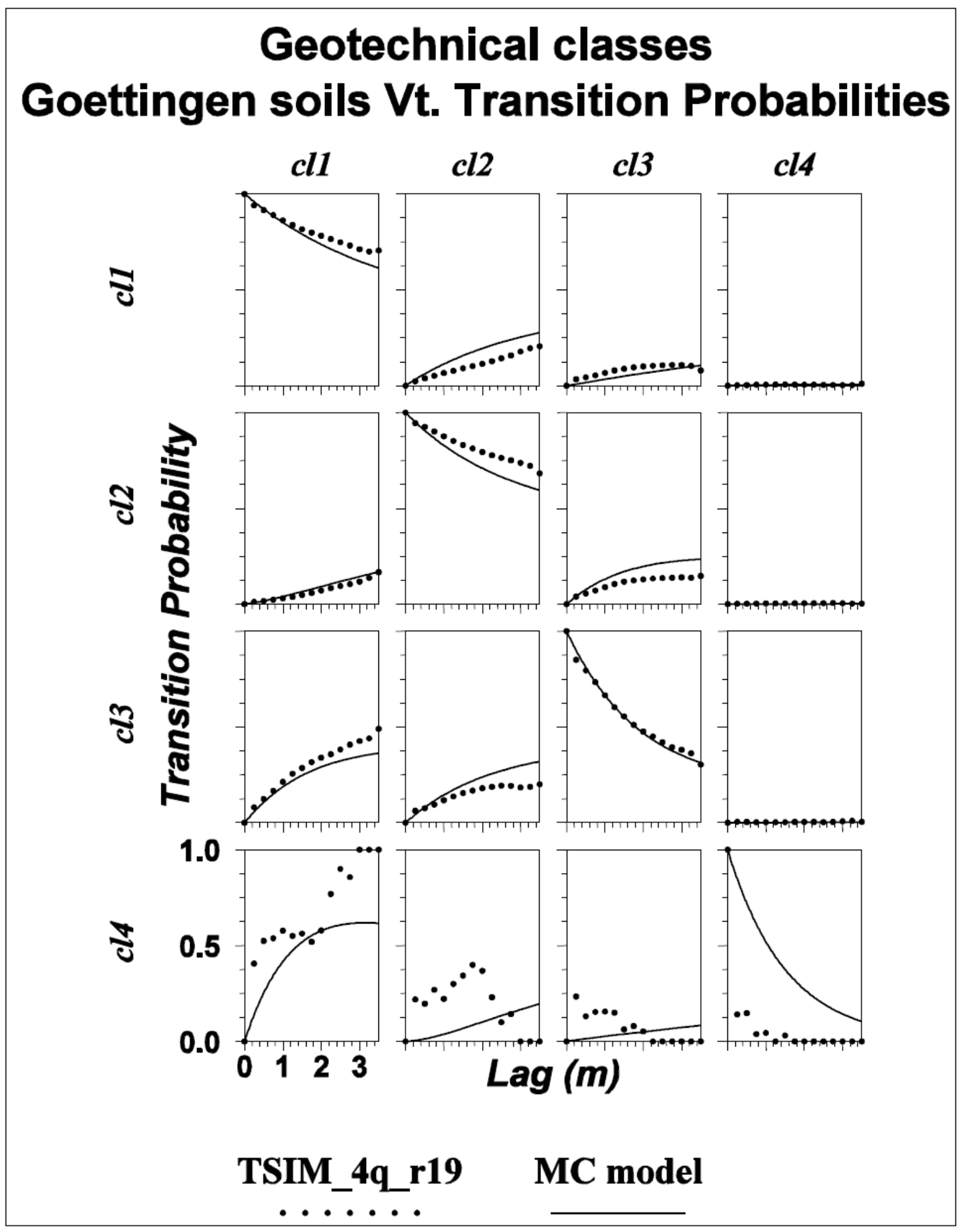

Figure 5.14 Vertical transition-probabilities among the soil classes for realization 19 generated by TSIM method with a four-step post-quenching phase (dots) and corresponding Markov chain models (solid lines). 


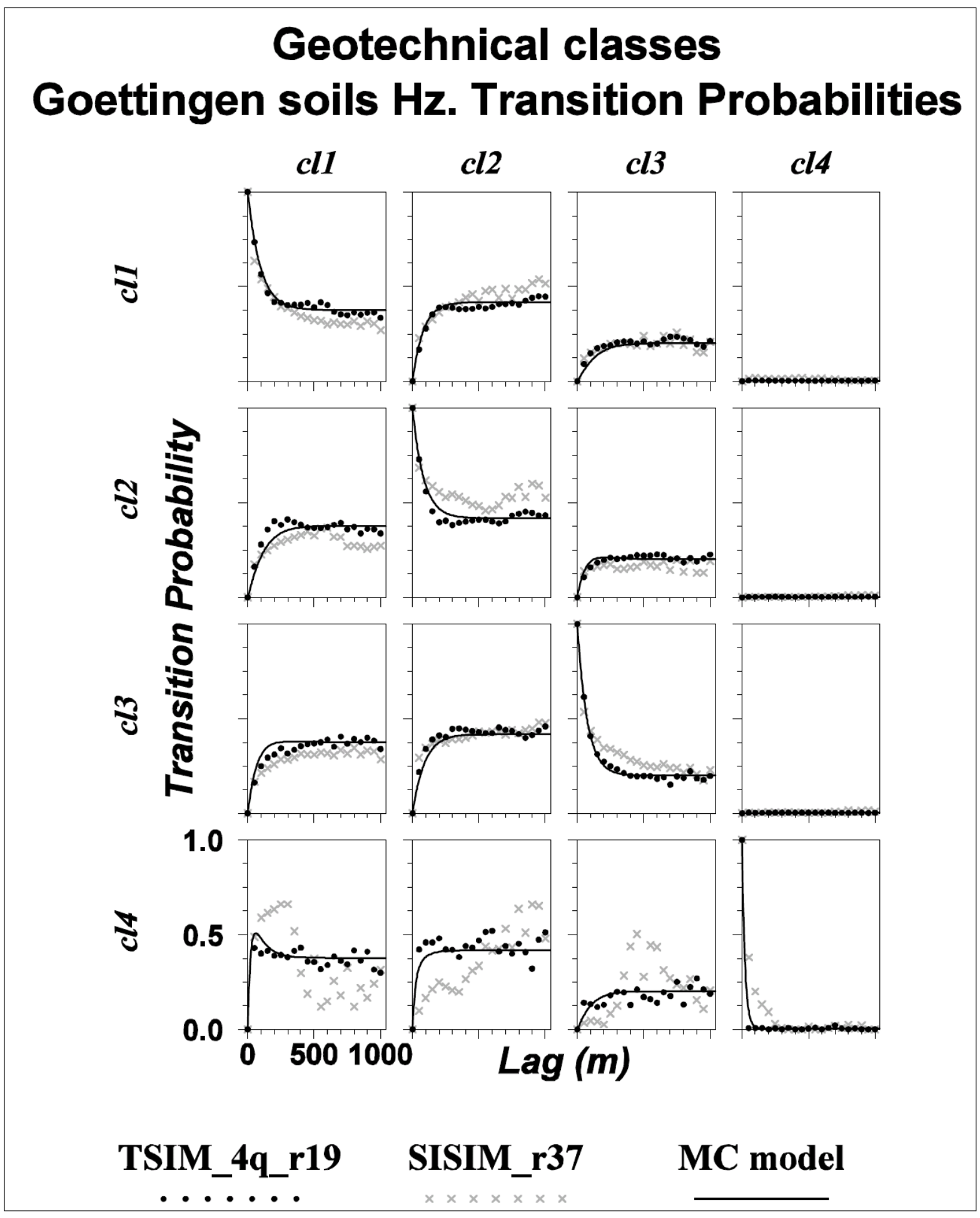

Figure 5.15 Horizontal transition-probabilities among the soil classes for realization 1 generated by TSIM method with a four-step post-quenching phase (dots), corresponding Markov chain models (solid lines), and realization 37 generated by SISIM method (cross symbols). 


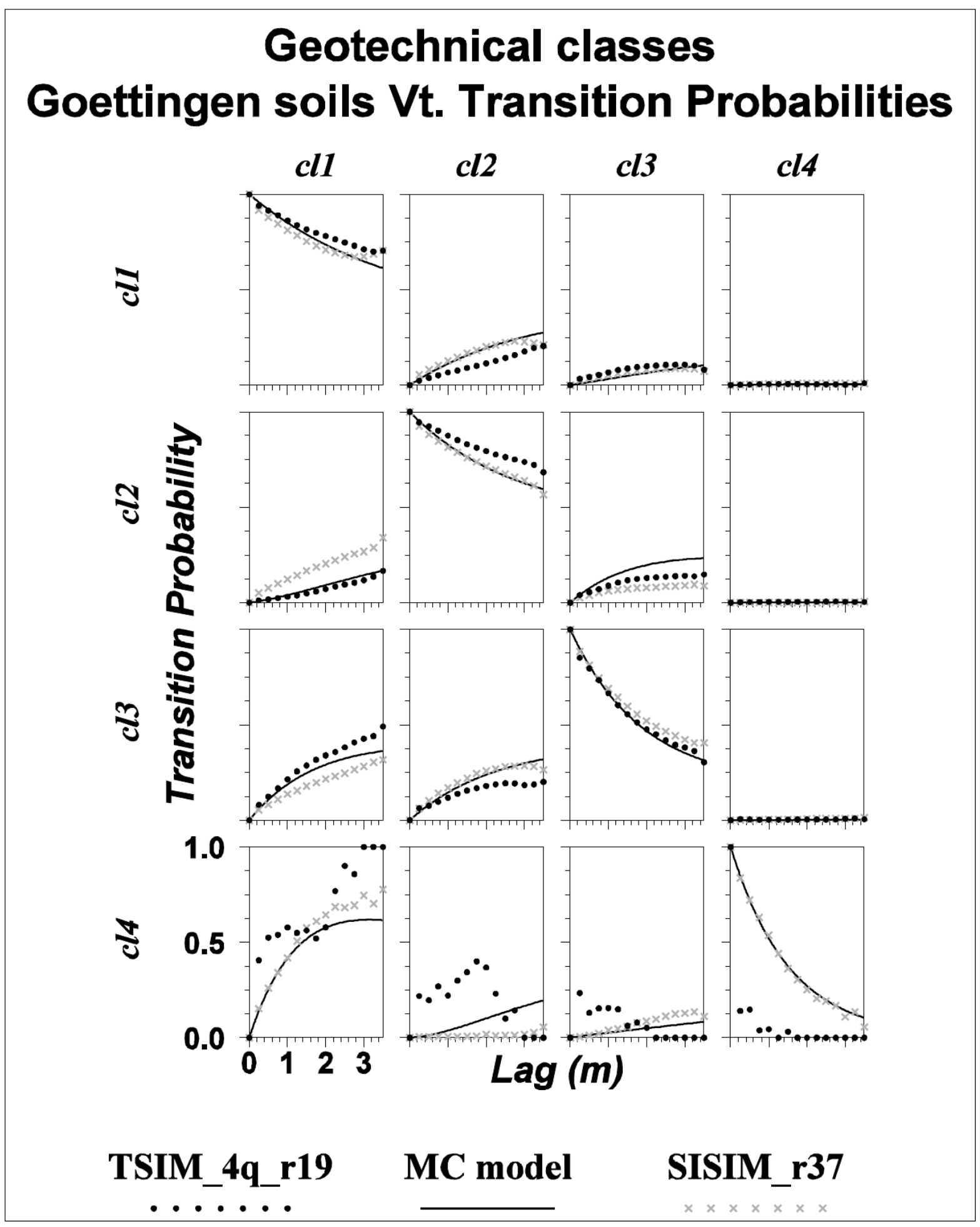

Figure 5.16 Vertical transition-probabilities among the soil classes for realization 1 generated by TSIM method with a four-step post-quenching phase (dots), corresponding Markov chain models (solid lines), and realization 37 generated by SISIM method (cross symbols).

For the TSIM method without any further quenching steps, the realization number 4 was selected because of its best proportions-reproduction. For this realization, the TPs usually 
reach a plateau, quicker than the model (Figure 5.7and Figure 5.8). The TP sills are also often different with the model. In this case, the number of near the origin points falling on or very close to the model line, is not so many, especially for the vertical direction. According to the Figure 5.9 and Figure 5.10, with a further quenching step in the TSIM method, the number of mentioned points (falling on or near the model line) and their corresponding closeness, in general, were improved in selected realization (i.e. realization number 19). The only exception was for the vertical TPs of the class 4 to any other classes that seem to become worse after quenching step though the difference were not too much. The mentioned general improvement trend by adding the quenching steps was continued for the TSIM simulations with two and four further steps, as well. In the TSIM with four post-quenching stages, the TPs-reproduction became worse in some cases and the improvements were not considerable. A logical conclusion could be that the number of quenching steps that improves the simulation TPs-reproduction should be optimize. The reason is that the TPs-reproductions is not improved considerably after some quenching steps but it can slightly affect the vertical TPs-reproduction of the class 4 with a considerably longer computational time.

The Figure 5.15 and Figure 5.16 compare the TPs-reproduction of the selected realizations of a four-step quenching TSIM method, and SISIM, with their corresponding Markov chain models. In general, it is clear that the TPs-reproduction of the TSIM method was considerably better except for some of the vertical TPs of the class 4 .

From Figure 5.15 and Figure 5.16, it can be inferred that except about the vertical transitionprobabilities of the soil class 4, the transition-probabilities of the selected realization (realization 37) of the SISIM method, was weaker than that of the TSIM methods.

\subsubsection{Variograms-reproduction of the transition-probability Markov chain simulations}

As one of the minimum indexes to assess the acceptance and goodness of the spatial variability structure-reproduction of the simulations evaluation, variograms can be applied besides the transition-probabilities.

To evaluate the model variograms-reproduction of the simulations, the following six key elements, were examined and evaluated; one for evaluating the set of realizations, and the five rest to assess the reasonability of each realization, separately. These elements included; (a) the extent of the scattering of the variogram lines in graph plane to evaluate 
the sets of realizations, and; (b) the general shape, (c) range, (d) sill, (e) nugget effect, and finally, (f) general behavior and slope of the variograms near the origin to evaluate each realization, separately.

Due to the higher probability (higher frequency) of coincidence of more frequent categories (bigger classes) with the drilling boreholes, the inferred variograms of the most frequent classes from the samples could be considered more reliable, and hence, their variograms and proportions-reproduction as a more emphasized acceptance condition. This fact is considered during the interpretations of the variograms and transitionprobabilities reproduction as well as histogram-reproduction of the simulations.

Figure 5.17 to Figure 5.19 summarize the horizontal and vertical variograms of different realizations for TP/MC method (TSIM simulations) and their corresponding model variograms for all twenty produced realizations of each method.

The variograms were calculated for the simulation methods of TSIM without any further quenching step, then with only one additional quenching step, and finally with two postquenching steps. These calculated simulation variograms were compared to their models. The general conclusions about the variograms-reproduction of the realizations of the TSIM simulation methods which can be drawn from these graphs as well Figure 4.3 (for the SISIM method) are summarized as following:

(a) TSIM had more similar variograms than those of SISIM especially after additional quenching steps. Adding the further quenching steps to the TSIM simulations for the vertical variograms of the all classes, the scattering of the variogram plots in the plotting area reduced. Likewise, for the variograms in main directions for class 4 , the dispersion of the variogram graphs of different realizations reduced after adding quenching steps. However, the horizontal variograms of the classes 1 to 3 represented slightly more dispersion after adding some further quenching steps. In general, it can be concluded that the dispersion extent of the variogram graphs of different realizations in the plot area was less than that of the SISIM method. This means that the TSIM simulation method, especially with added quenching steps has produced more similar variograms than what the SISIM method could.

(b) The overall variogram shapes and range considering the number of distinguishable structures and how quickly they reach their sills (not necessarily the expected sill), was quite similar to the model for vertical variograms especially after adding some quenching steps. For the horizontal 
simulation variograms on the other hand, they were somehow different. Although, considering the slopes of the second rise in the horizontal variograms, the simulation variograms with some quenching steps were slightly nearer to the model. The reason for considering the second rise is that the first part was speculated to reflect the short-range changes or the nugget effect parameter of the variograms.

\section{The Interpretation of the realization variograms of various TSIM simulation methods (with different quenching step numbers) :}

The general approach to interpret the simulation variograms, in this study, was based on the graphical assessments and comparisons of the simulation and model variograms of different soil classes in a range of directions for the spectrum of all realizations produced by different simulation methods. The variograms of all realizations produced by a specific geostatistical simulation method were plotted in a single graph to assess the dispersion of the variograms for all realizations of this method. In addition, in this graph, they could be compared with their corresponding variogram models to evaluate how good the realizations could reproduce the underlying variogram models. Figure 5.17 to Figure 5.19 represent the mentioned simulation and model variograms, each figure relevant to a TSIM geostatistical simulation method with no-, one-, and two-steps of post-quenching phases, respectively.

In general, the horizontal simulation variograms of the soil classes 1 to 3 , from their second rise, represent a kind of fluctuation resembling a hole-effect model. These fluctuations (for soil classes 1 to 3 ) were somewhat intensified after adding quenching steps.

This phenomenon could be interpreted as the occurrence of a kind of periodicity in such a simulation method. It should be considered that, the sample variograms and their corresponding fitted models reflect only an overall trend in the spatial variability structure especially due to the limited number of observations.

Therefore, the speculation could be that the available fluctuations did not necessarily signify an improper reproduction of the variability structure model. 

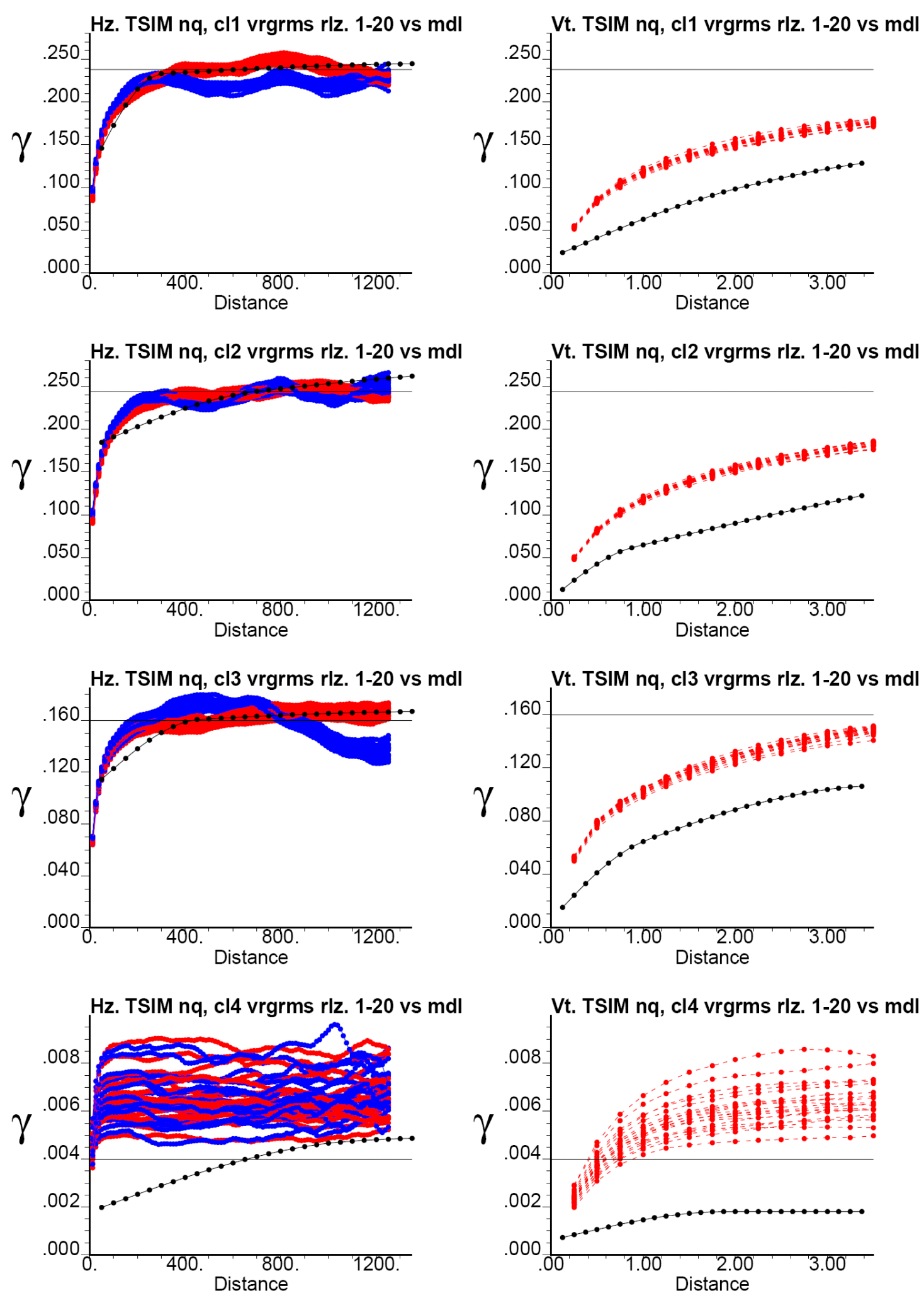

Figure 5.17 Indicator variograms of all 20 realizations generated by the TSIM simulation method without any quenching steps (colored lines), and their corresponding model variograms (black lines). The graphs on the left side represent the horizontal variograms (red for North-South, and dark blue for East-West directions), and the graphs on the right side represent the vertical variograms. 
Hz. TSIM 1q, cl1 vrgrms rlz. 1-20 vs mdl

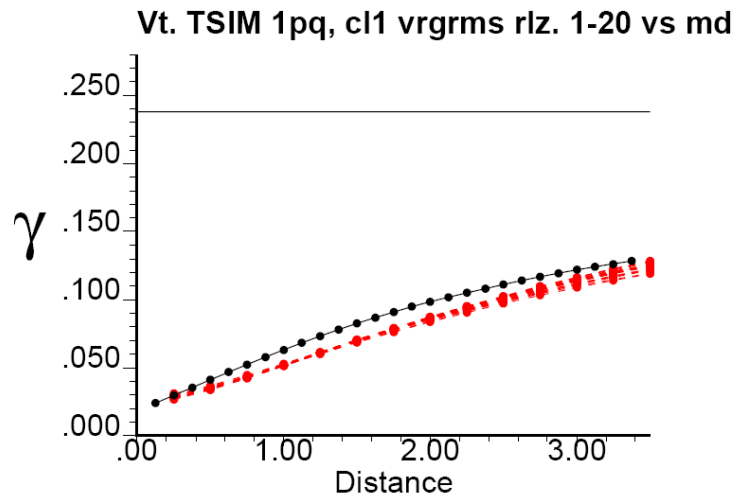

Hz. TSIM 1pq, cl2 vrgrms rlz. 1-20 vs md

Vt. TSIM 1pq, cl2 vrgrms rlz. 1-20 vs md
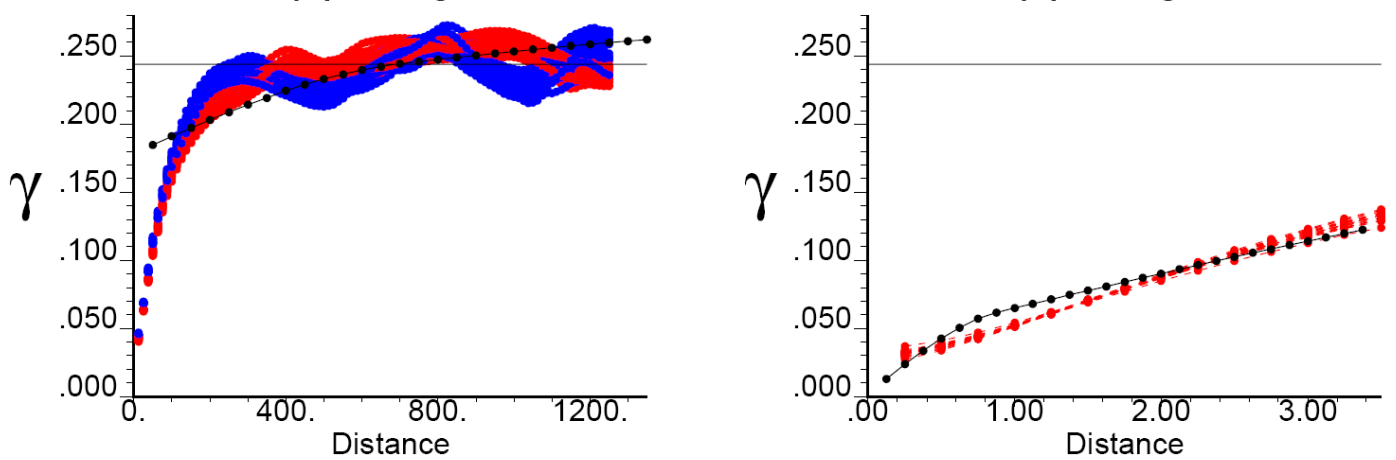

Hz. TSIM 1pq, cl3 vrgrms rlz. 1-20 vs md

Vt. TSIM 1pq, cl3 vrgrms rlz. 1-20 vs md
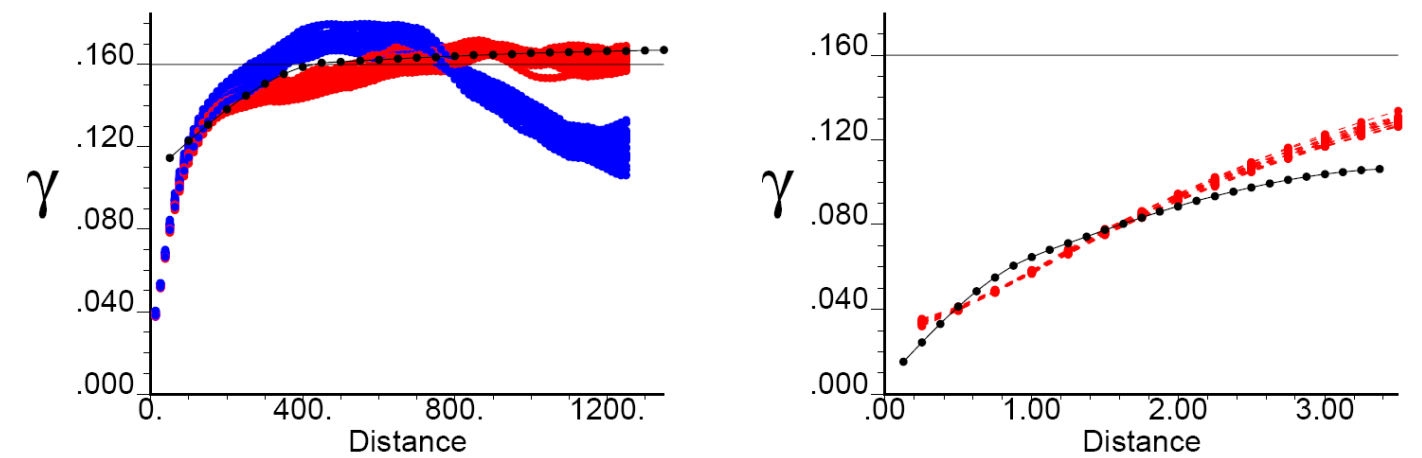

Hz. TSIM 1pq, cl4 vrgrms rlz. 1-20 vs md

Vt. TSIM 1pq, cl4 vrgrm rlz. 1-20 vs mdl
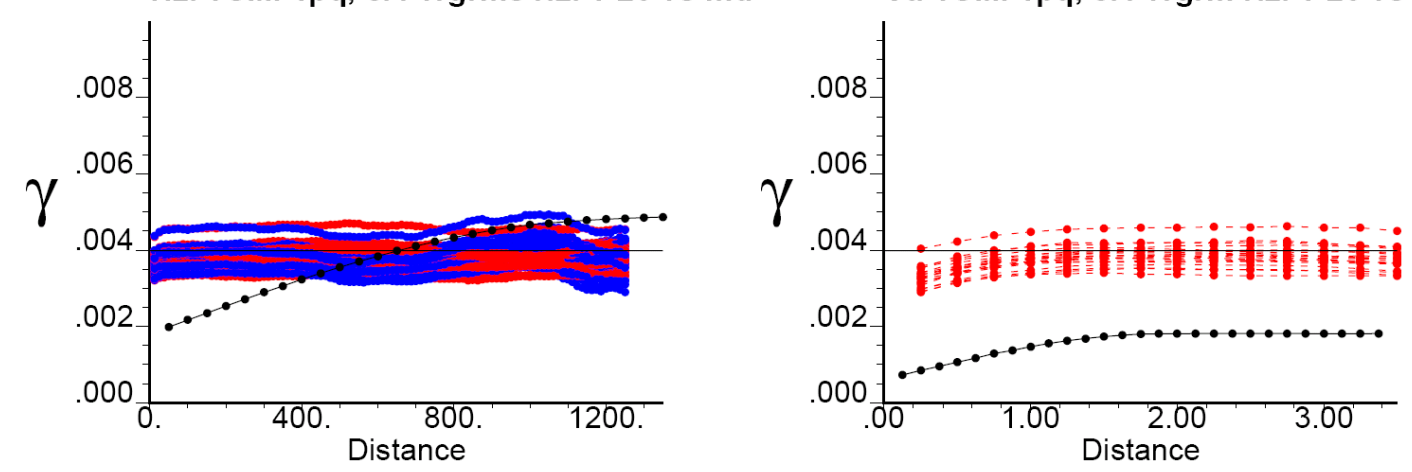

Figure 5.18 Indicator variograms of all 20 realizations generated by the TSIM simulation method with one post-quenching step (colored lines), and their corresponding model variograms (black lines). The graphs on the left side represent the horizontal variograms (red for North-South, and dark blue for East-West directions), and the graphs on the right side represent the vertical variograms. 

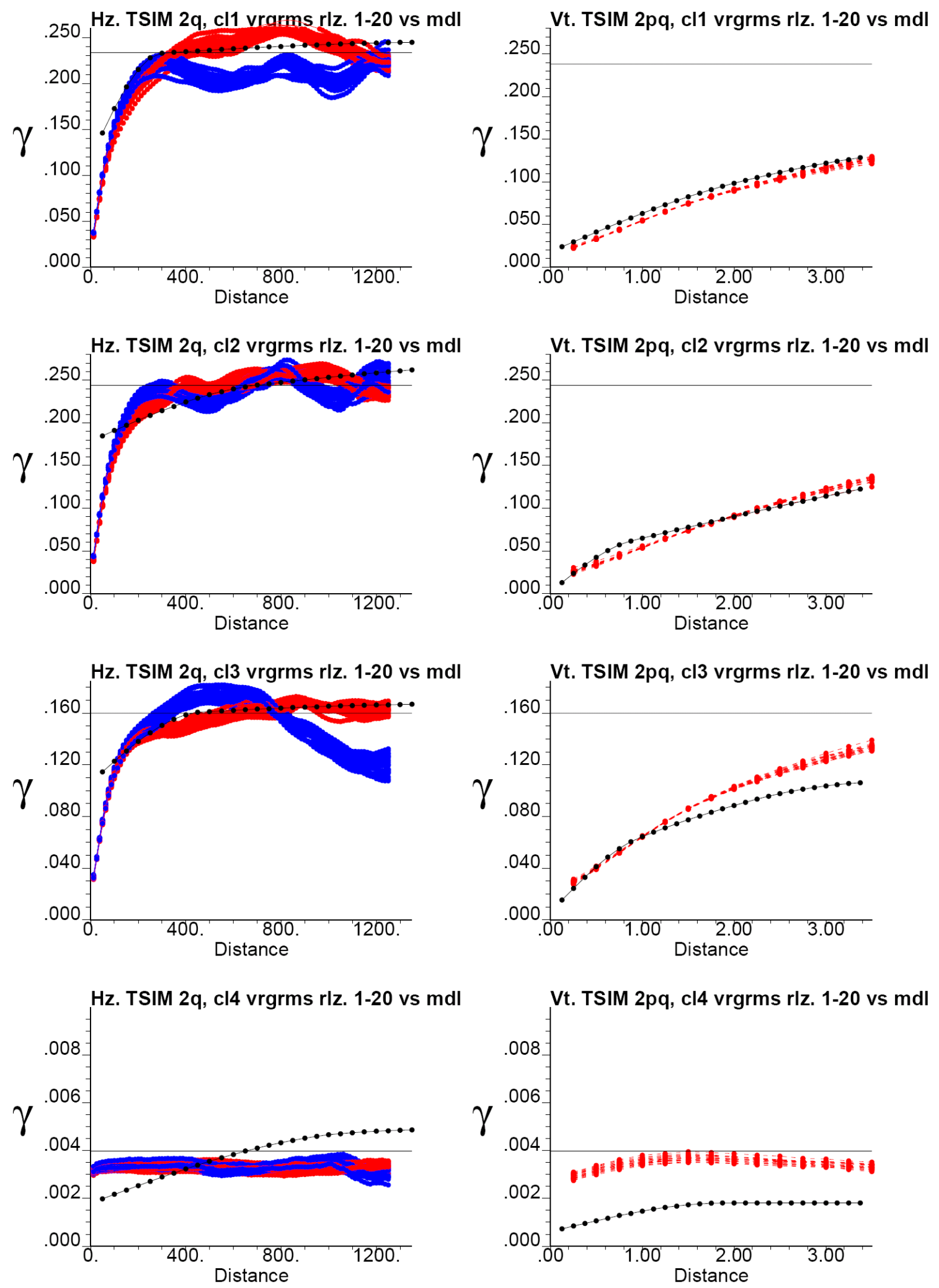

Figure 5.19 Indicator variograms of all 20 realizations generated by the TSIM simulation method with two post-quenching steps (colored lines), and their corresponding model variograms (black lines). The graphs on the left side represent the horizontal variograms (red for North-South, and dark blue for East-West directions), and the graphs on the right side represent the vertical variograms. 
In addition, the divergence between the variograms of the simulations in horizontal North-South and East-West directions somewhat increased in the TSIM simulations after conducting some quenching steps, except for the variograms of the soil class 4 . For the TSIM simulations without any quenching step, the variograms reached their sills in a shorter range than those with quenching steps (especially for the horizontal variograms of the soil classes 2 and 3) and, as mentioned before, the fluctuations were less, except for the simulation variograms of class 4 . Closing the eyes to these fluctuations, the overall tendency of these horizontal variograms with further quenching steps, except about class 4, was to reach their sills (but not exactly their expected sills), more or less at the same distances as those of the model variograms. In addition, in the second rise of the simulation variograms of the simulations with or without quenching steps, the beginning part of the graphs (nearer to the origin) represented a quicker rise than those of the model. The figures represent that the realization variograms are clearly closer to each other in shorter distances and they diverge more when the distance is increased. Therefore, their shorter-scale structures should expectedly be more similar than their long-distance structures.

Figure 4.1 to Figure 4.3 and Figure 5.17 to Figure 5.19 represent the SISIM and TSIM realization variograms, respectively. Considering these figures, the behavior of their realization variograms with each other and with their relevant variogram models can be compared. It seems that, the TSIM realizations represent closer overall shapes to the model than those of the SISIM method, in terms of their variograms.

In regard to variogram interpretations, two additional comments deserve pointing out here:

(1) The interpretation of the variogram nugget-effects in simulation variograms was not that straightforward. Considering the intercepts of the variogram lines and the vertical axis of the variogram graphs as the nugget effect, the nugget effects of the simulations would apparently be much lower than those of the model. These nugget-effect differences between the simulations and model variograms of soil classes 1 to 3 might be due to either the low variations in very short-scale of the simulations (i.e. a higher continuity) or the shortage of enough observations for approximating the sample variograms from input data. However, bearing in mind that the nugget-effect of a variogram is defined as the 
representative of a variogram value in very short-distances, it could be concluded that the first part of these variograms, which show the first quick rises and are corresponding to very short distances, could be considered as a representative for the nugget-effect value. A distance equivalent to the two first lag-distances of the model variogram was suggested as this short-range distance here. Hence, it was suggested in this study to consider a range of just less than 100 meters for the horizontal, and less than 0.4 meters for the vertical variograms as the nugget effect range. This distance is in the range of the observed first section of every variogram which represent very quick rises. With such an insight to the nuggeteffect issue, variogram nugget effects of the TSIM simulations without quenching steps were jus a bit more than those of the model. The nugget-effect became closer to that of the model after conducting some quenching steps on the TSIM simulations. About class 4, the nugget effects are considerably more than the model. This phenomenon could be due to the reason that, the short-scale variations of the simulation variables in horizontal direction were so high that it produced a much bigger nugget effect than that of the model. In fact, the simulation variograms for class 4 is very much like a pure nugget effect model that means the kriging estimation would act very much like a typical interpolation. Compared to the SISIM variograms, the difference of the simulation variogram nugget-effects among different realizations was much less in the TSIM method.

(2) The sills in average seem to be fairly close the expected (or model) sills in horizontal variograms of the TSIM method either with or without quenching steps, though about the class 4 , the difference were more. About the TSIM simulations without quenching steps, the vertical variograms of all soil classes and horizontal variograms of the soil class 4 , the sills were more different from those of the model. These sills got closer to the model and their variations decreased after performing quenching steps on the initial TSIM simulations without any quenching step. Compared to the SISIM variograms, their variations were less and, in general, the sill reproductions were better in the TSIM method. 
The general conclusion about the variogram reproduction of the TSIM method could be that the variogram reproduction of TSIM method especially with quenching steps was noticeably enhanced compared to that of the SISIM method.

\subsubsection{Geological soundness:}

All the models here were constructed, on the one hand, based on the specific conditioning data from drillings and identified geostatistical characteristics that are capable of reflecting underlying geological characteristics. But on the other hand, the non-exhaustive sampling can cause the missing of a wide range of information about data and variability inside the model. Therefore, all the facts and details about the geology of the model for unsampled locations within the study zone could not be captured by existing boreholes. Although the geostatistical estimation and simulation methods rely basically on the geostatistical assumptions and schemes, some geological facts still should be taken into account within their framework in different ways. For instance, the separation of the stationary zones, decisions about variogram model parameters (such as nugget effect, anisotropy directions, etc), and estimation parameters (such as search radius, etc) are among the factors that take the geological information and interpretation of the experts into the consideration which can be included in the geostatistical models by geological considerations. Some methods such as transition-probability Markov chain simulation method (TP/MC) facilitate the integration of geological and physical information into the geostatistical models. In the TP/MC method, some geological information such as mean length, and juxtapositioning patterns of lithofacies can also be included in modeling. However, the geostatistically generated models still need verification for their geological soundness in addition to checking their geostatistical and statistical consistency to ensure their consistency with the geological interpretations and expectations. Thus, one of the most important minimum criterions of the acceptance of geostatistical simulations is their geological soundness.

However, to the extent of author's knowledge and investigation, straightforward quantitative criterions to evaluate the geological acceptability of the geostatistical simulations have not been represented in the literature relevant to the geostatistical or comparable techniques. Most of the available geological evaluation criterions are simply subjective and interpretive. In addition, since the modeled classes are geotechnical-based, 
it is not straightforward to make a close link between the generated geotechnical simulations and geological interpretations. Though fortunately, the available geotechnical classes in these models convey an extent of geological meaning since the average composition of their particle size is getting finer from the geotechnical soil class 1 to soil class 3 while the mineralogical composition of the soil classes is also considered. In other words, soil class 1 contains the coarsest particles (including gravel, sand and their mixtures) whereas the class 3 (including clay and silt) has the finest particles. The soil class 4 falls in the organic materials category while it is a very minor constituent of the gathered samples. Subsequently, the expected frequency of the occurrence of class 4 in the model would also be very low.

The quaternary sediments construct this sedimentary set and can be investigated as a fluvial system. The river has flown and slowed down along the valley bed and deposited the existing sediments. These deposits could reflect various patterns of the fluvial systems such as probable river-bed changes, braided river forms, and meanderings during their sedimentation course which could be combined with other sedimentation features. The changes in the river-bed location and its elevation were expected and could be identified in the model.

The sedimentation paths, directions, and locations may change continually to form a more complex sedimentary structure, due to the variations in the river-bed.

Some observed patterns in the simulation models resemble a meandering or braided river system.

A meandering system is formed when the flowing water in a stream erodes the banks of the river and widens its valley. Formation of such a structure happens on an almost level landscape and where the banks of the stream can easily be eroded. ${ }^{1}$

In a meandering system, the coarser sediments are deposited where the flow slows down inside the river path curves and the finer sediments are deposited in the opposite side. Therefore, the expected geometry of the coarse sediments accumulations could be a set of channel-like features.

According to the simulation results (Figure 5.20 to Figure 5.34), the coarse sediments occur mainly in the middle parts of the valley.

\footnotetext{
${ }^{1}$ Some example references defining the meandering are listed here; "Meander (river System Component)." Encyclopedia Britannica Online. Encyclopedia Britannica. Web. Jan. 2012; "Meander." Wikipedia. Wikimedia Foundation, Winter 2011. Web. Jan. 2012. http://en.wikipedia.org/wiki/Meander; "Meander" in "Geological terms beginning with 'M'," from Dictionary (Geology and earth science terms and definitions), Website of geology.com, [On-line]. Available: http://geology.com/dictionary/glossary-m.shtml.
} 
Some attempts to evaluate the geological soundness of the selected realizations were conducted here considering the statistical measures including the transition-probabilities and variograms which can consider valuable extent of geological information in the model.

The essential points about the geological assessments of simulation models can be summarized as following:

(1) In the selected simulations, the predominant soil category observed in the middle of the basin is class 1 which is the representative of a less wellsorted sedimentary group and contains the coarsest particles and comprises the coarsest average grain size. The geometry and location of class 1 masses in the model give the impression of different probable sedimentary features such as the paths of riverbed and their changes, meandering, braided rivers system, and so on, with changing and accumulating sediments processes.

(2) An extent of connectivity and layering is obvious in masses of different categories in the simulation model which can resemble sedimentary system characteristics. This feature is better generated in the TSIM simulations. The Walther's law of facies succession suggests that the vertical sequence of facies reflects the lateral changes of the facies in the lack of nonconformities (Leeder 1982, p. 122). Hence, the TPs1 (and their corresponding models) can be used to recognize the neighboring categories, especially for the more reliable vertical directions (Figure 5.3 and

(3) Figure 5.4). According to these graphs, the class 2 is the most likely class to occur beside the class 1 while the class 3 is a little less likely to be adjacent to the class 1 although its probability is still not that low. The geological occurrence of the class 3 (the finest material) beside the class 1 (the coarsest composition), as it was also very probable according to the transitionprobabilities, could be due to different reasons such as the rush of the finer sediments from the basin sides to the middle of the basin during the erosion and sedimentation. Another probable reason could be that the river side is usually adjacent to the fine materials where the river current border is finished (see the rightmost side of the Figure 5.26 and Figure 5.27). In

\footnotetext{
${ }^{1}$ TPs in this document, stands for the transition probabilities
} 
addition, a meandering stream can leave the coarse and fine sediments in two opposite side. Naturally, the intermediate sized compositions could usually be accommodated somewhere in between. These fine materials (class 3) near the slope sides sometimes occur above the coarse compositions (class 1) which can geologically be a plausible feature. This phenomenon is observed more in the eastern side of the basin.

(4) Very limited samples of organic materials (class 4 in this model) have been observed in samples and hence they have occupied a very minor volume of the model. The source of organic materials in the zone of model could be due to various reasons. For example, they might have been brought from the upper layers to the lower levels during the drilling and sampling course. Obviously, the upper layers are closer to the zones of warmer sedimentation periods of deposition and are more likely to contain organic materials. They also can belong to the transition zones to the upper layers with more probability of containing the organic sediments. Even they might have been recorded due to the misinterpretation of some samples especially since they only constitute a limited number of samples and they can be resembled and mistaken with similar sediments of the same appearance ${ }^{1}$.

The general conclusion that might be drawn from the above discussion could be summed up as following:

(1) No considerable objection against the geological soundness of generated simulation models could be identified here.

(2) The geological reasonability of the TSIM simulation was even better than that of the SISIM simulations due to the weaker layering structure and more elongations of the material bodies in the vertical direction.

Hence, the models can be initially accepted and considered for further evaluations. The attention could be paid that such a model with a connected zones of coarse alluvial sediments (like what there is here for class 1), can act as a better flow medium for the fluid flows that might be underestimated in the disseminated patterns of the SISIM simulations. This point could become very important in subsurface flow simulations or geotechnical evaluations. Such a less-disseminated pattern also seems closer to the

\footnotetext{
${ }^{1}$ As mentioned before, the characterization and classification of the samples were mainly based on interpretive methods.
} 
geological realities of the comparable fluvial systems. Figure 5.34 to Figure 5.36 represent how adding the quenching steps in TSIM method can increase the geological continuity.

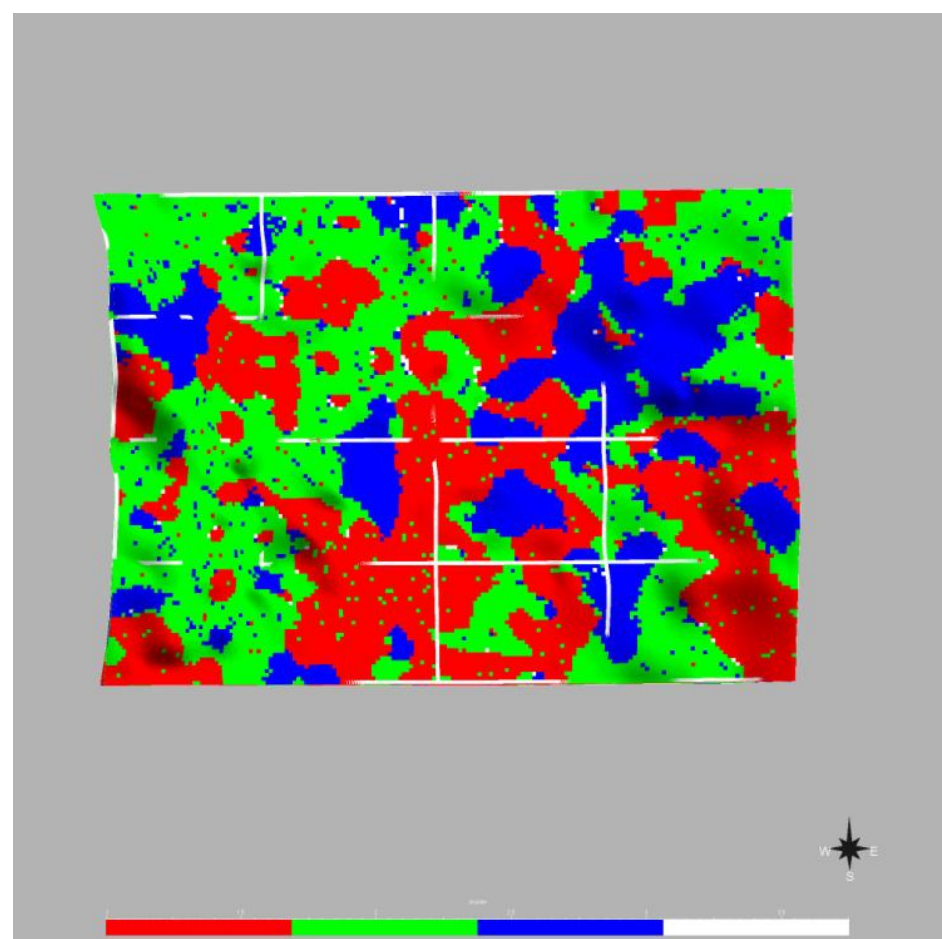

Figure 5.20 A perspective top view of the realization 12, generated by the TSIM with two postquenching steps simulation method. The model represents a $2180 \mathrm{~m}$ distance in EW and a $1580 \mathrm{~m}$ distance in NS direction. 


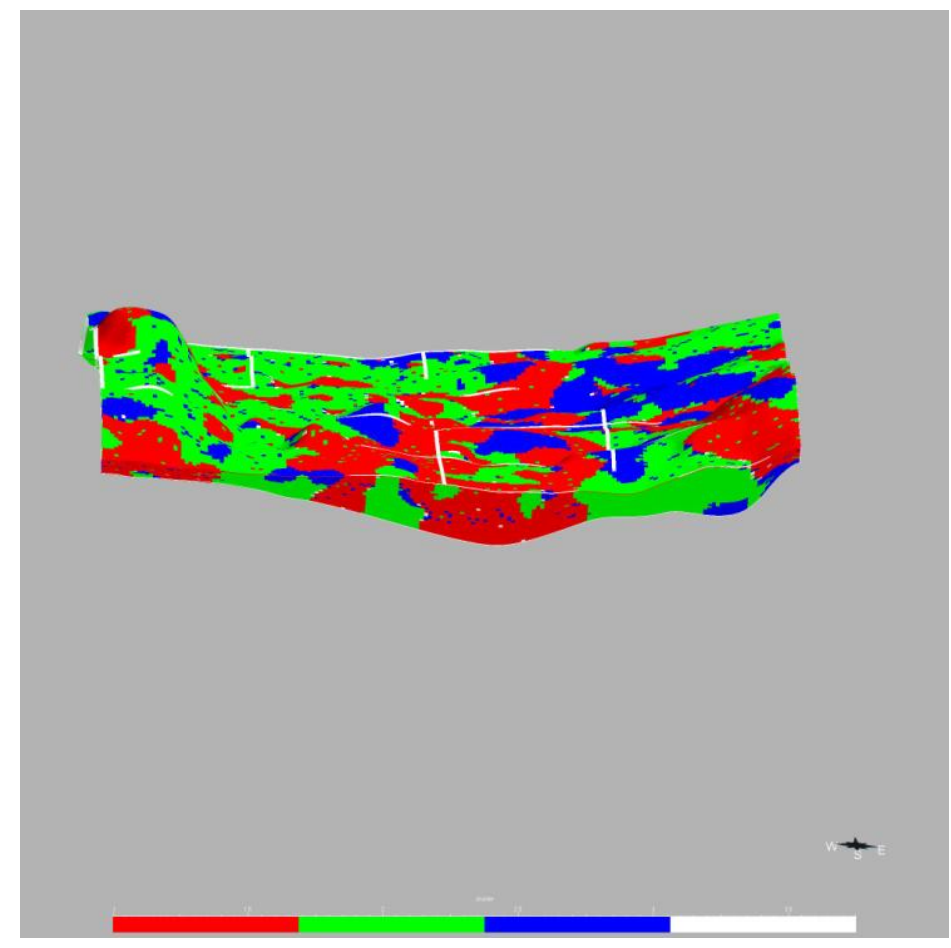

Figure 5.21 A perspective side view of the realization 12, generated by the TSIM with two postquenching steps simulation method. The model represents a $2180 \mathrm{~m}$ distance in $\mathrm{EW}$ and a $1580 \mathrm{~m}$ distance in NS directions with 15x exaggeration in the vertical direction.

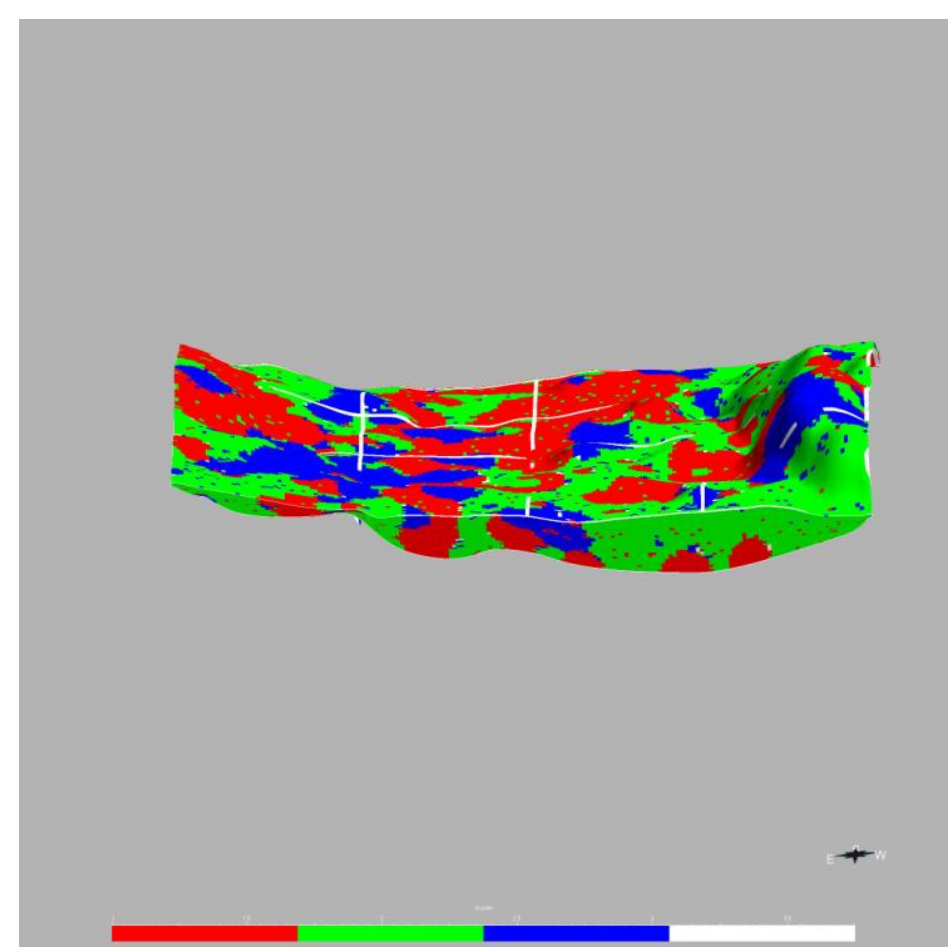

Figure 5.22 A perspective side view of the realization 12, generated by the TSIM with two postquenching steps method. The model represents a $2180 \mathrm{~m}$ distance in $\mathrm{EW}$ and a $1580 \mathrm{~m}$ distance in NS directions with 15x exaggeration in the vertical direction. 


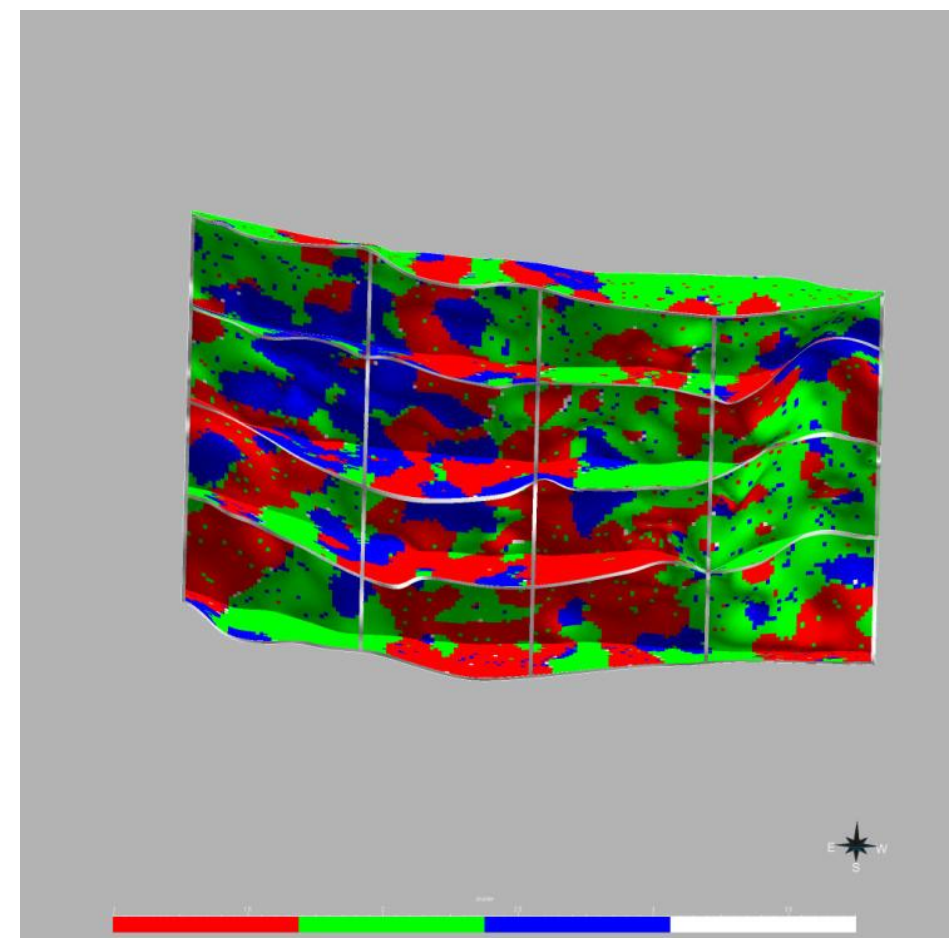

Figure 5.23 A perspective bottom view of the realization 12, generated by the TSIM with two postquenching steps simulation method. The model represents a $2180 \mathrm{~m}$ distance in $\mathrm{EW}$ and a $1580 \mathrm{~m}$ distance in NS directions with 15x exaggeration in the vertical direction.

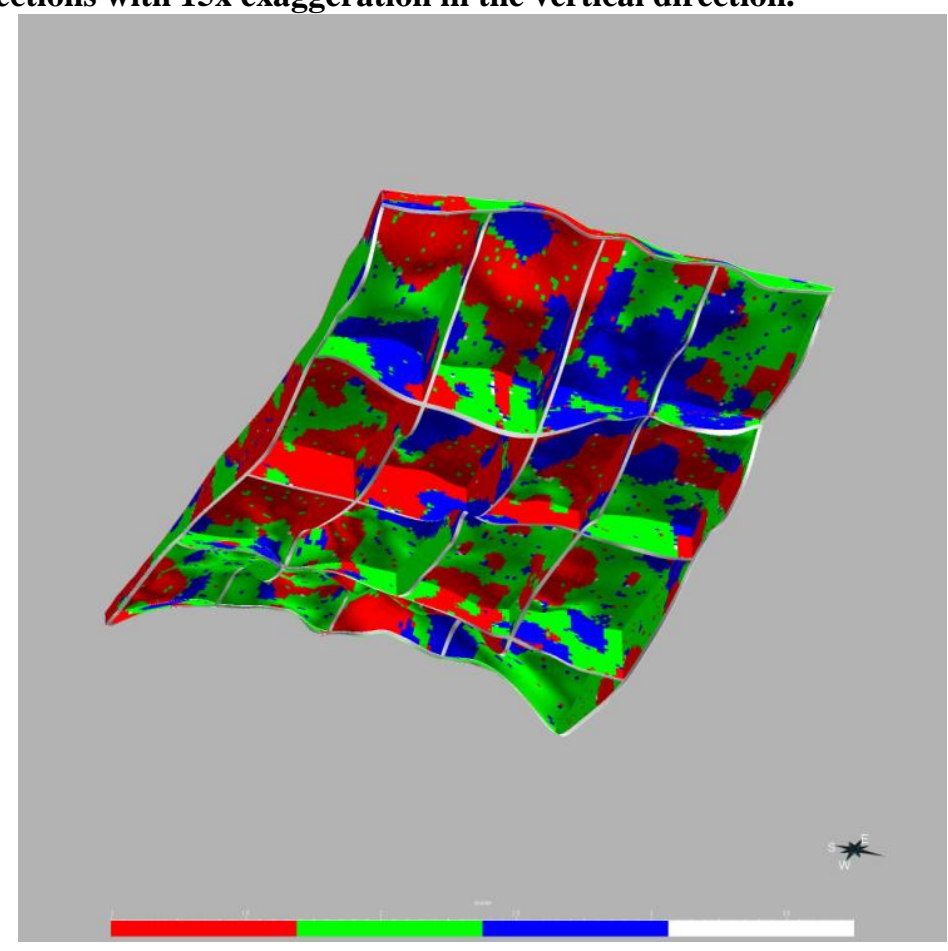

Figure 5.24 A perspective bottom view of the realization 12, generated by the TSIM with two postquenching steps simulation method. The model represents a $2180 \mathrm{~m}$ distance in $\mathrm{EW}$ and a $1580 \mathrm{~m}$ distance in NS directions with 15x exaggeration in the vertical direction. 


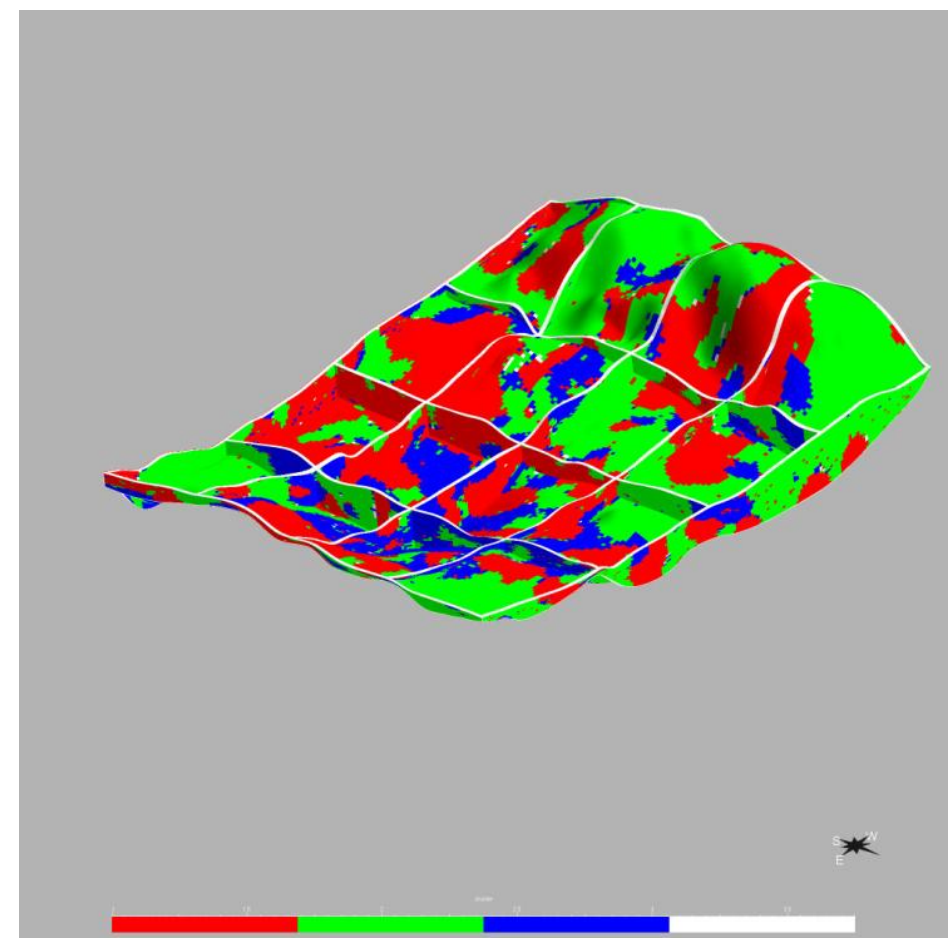

Figure 5.25 A perspective bottom view of the realization 12, generated by the TSIM with two postquenching steps simulation method. The vertical slice 21 has been depicted. The model represents a 2180m distance in EW and a 1580m distance in NS directions with 15x exaggeration in the vertical direction.

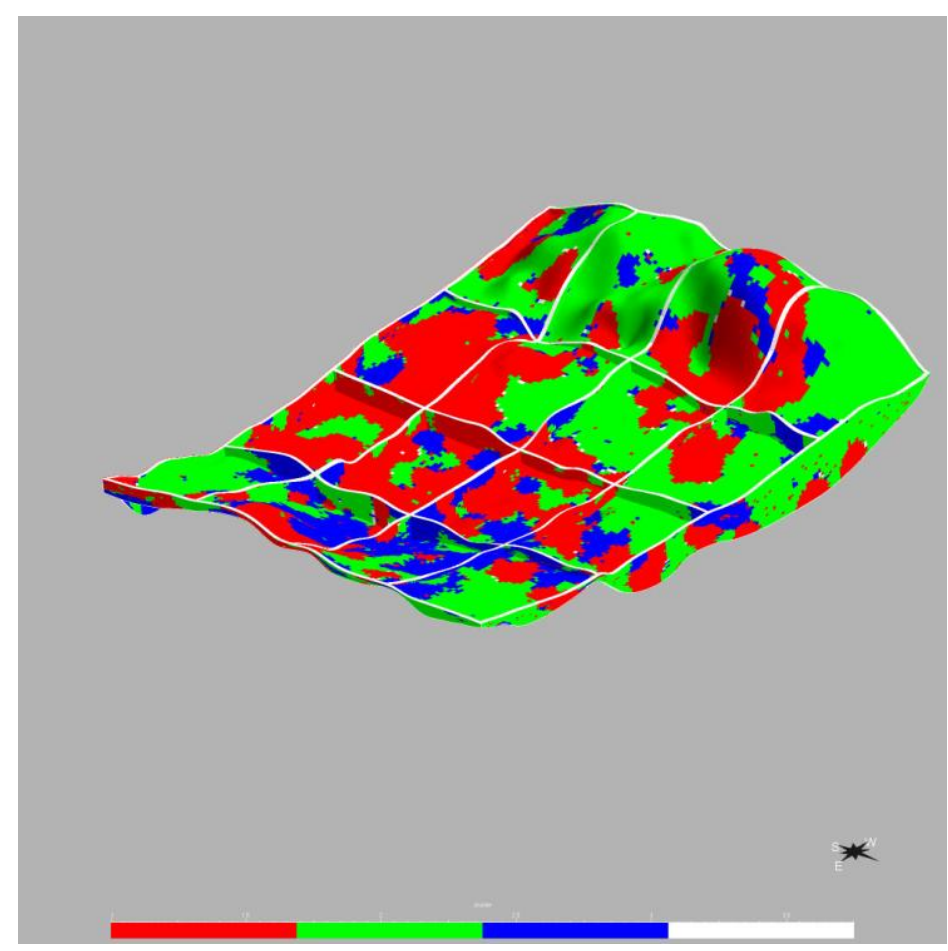

Figure 5.26 A perspective bottom view of the realization 12, generated by the TSIM with two postquenching steps simulation method. The vertical slice 14 has been depicted. The model represents a $2180 \mathrm{~m}$ distance in $\mathrm{EW}$ and a 1580m distance in NS directions with 15x exaggeration in the vertical direction. 


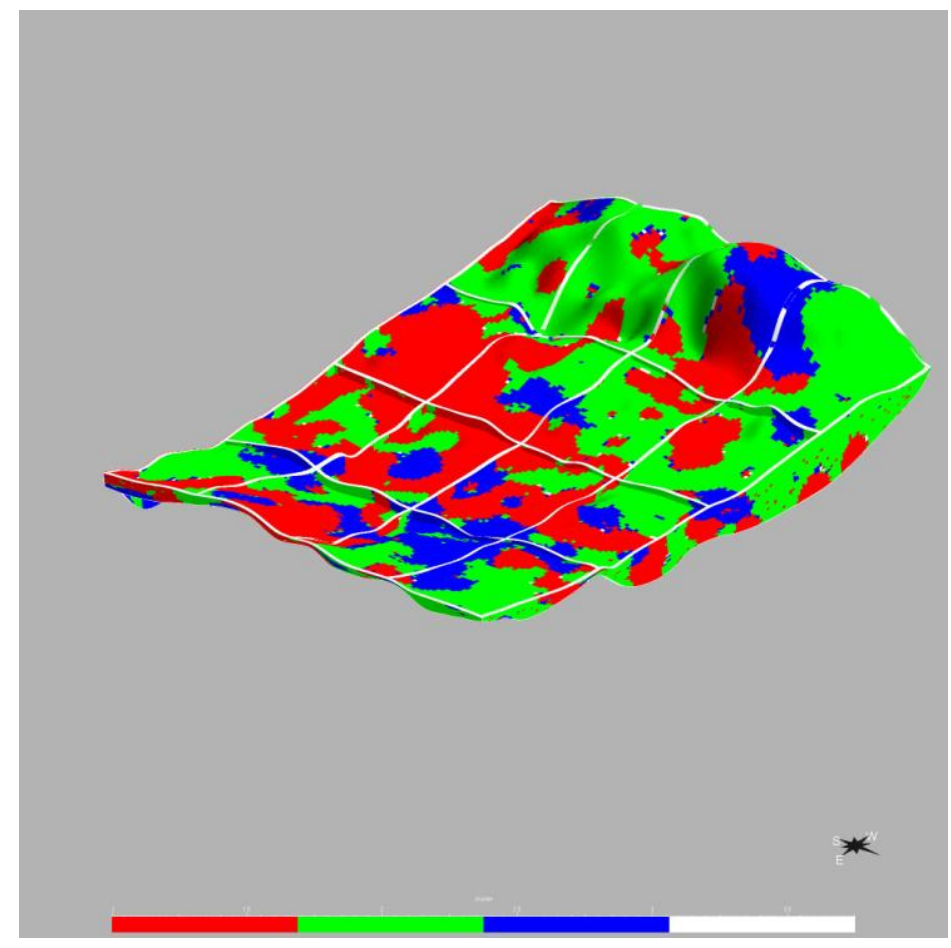

Figure 5.27 A perspective bottom view of the realization 12, generated by the TSIM with two postquenching steps simulation method. The vertical slice 7 has been depicted. The model represents a 2180m distance in EW and a 1580m distance in NS directions with 15x exaggeration in the vertical direction.

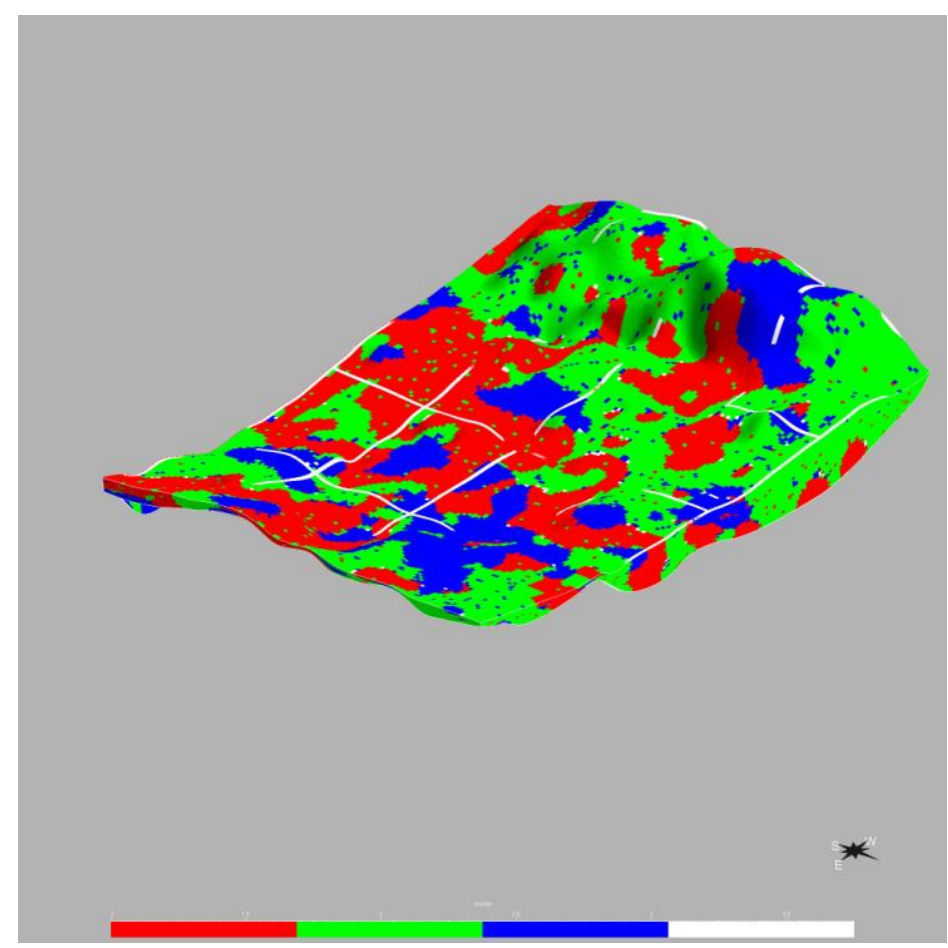

Figure 5.28 A perspective bottom view of the realization 12, generated by the TSIM with two postquenching steps simulation method. The vertical slice 2 has been depicted. The model represents a $2180 \mathrm{~m}$ distance in $\mathrm{EW}$ and a $1580 \mathrm{~m}$ distance in NS directions with $15 \mathrm{x}$ exaggeration in the vertical direction. 


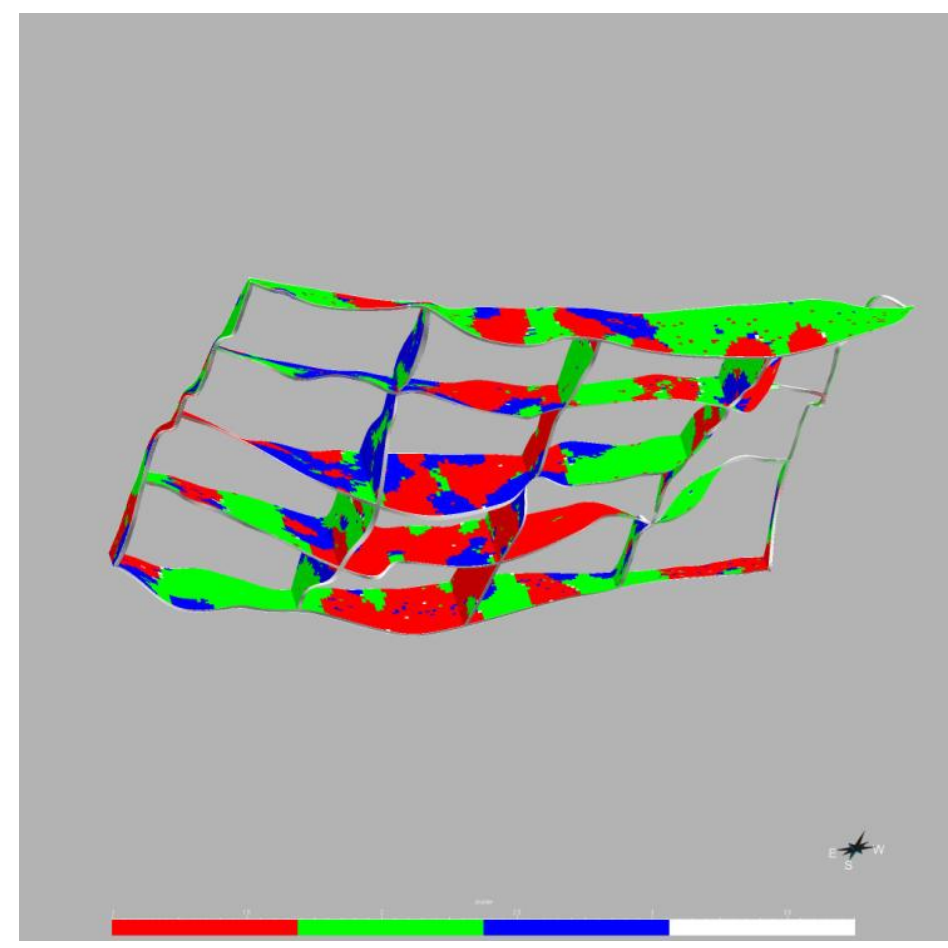

Figure 5.29 A perspective fence-model bottom side view of the realization 12, generated by the TSIM with two post-quenching steps simulation method. The vertical slice 2 has been depicted. The model represents a $2180 \mathrm{~m}$ distance in $\mathrm{EW}$ and a $1580 \mathrm{~m}$ distance in NS directions with 15x exaggeration in the vertical direction.

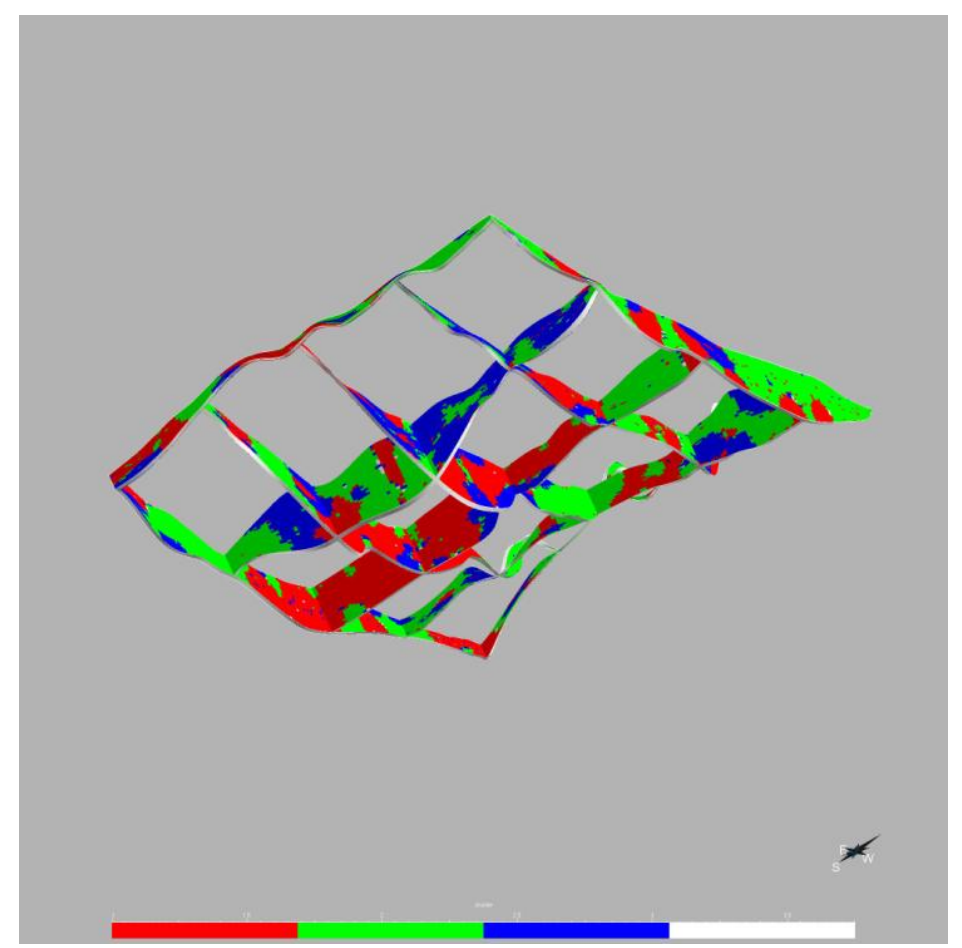

Figure 5.30 a perspective fence-model bottom view of the realization 12, generated by the TSIM with two post-quenching steps simulation method. The vertical slice 2 has been depicted. The model represents a $2180 \mathrm{~m}$ distance in $\mathrm{EW}$ and a $1580 \mathrm{~m}$ distance in NS directions with $15 \mathrm{x}$ exaggeration in the vertical direction. 


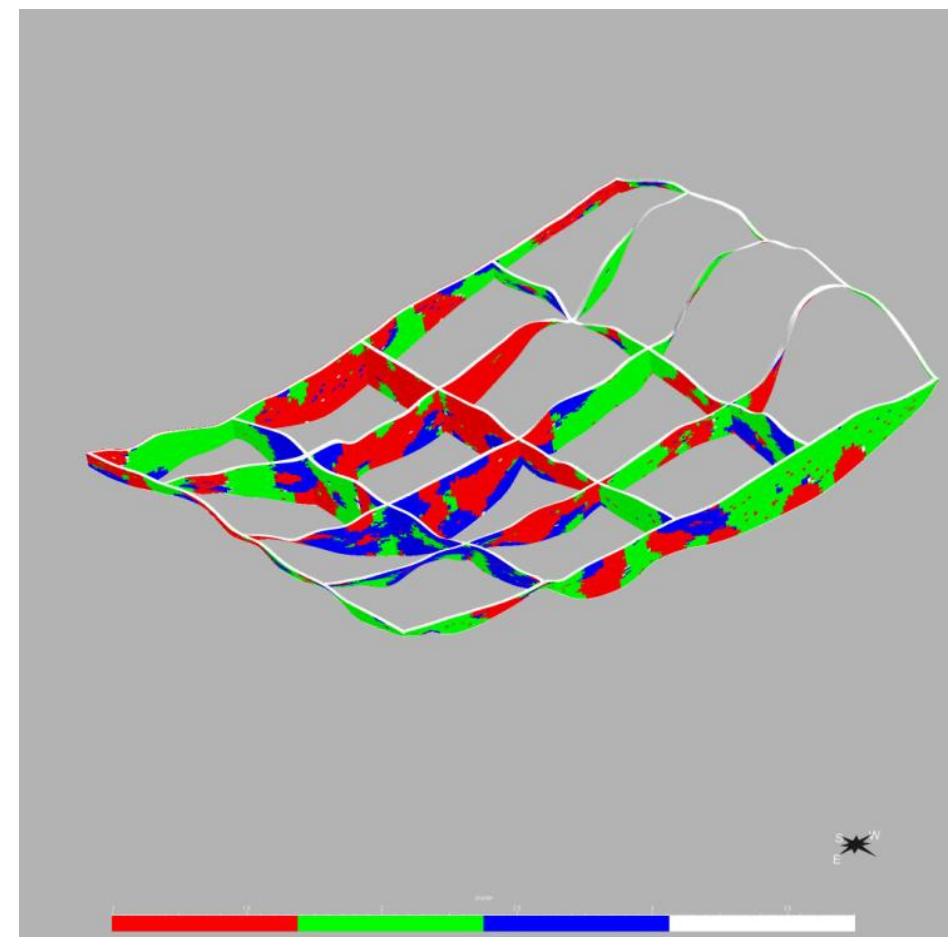

Figure 5.31 a perspective fence-model top view of the realization 12, generated by the TSIM with two post-quenching steps simulation method. The vertical slice 2 has been depicted. The model represents a $2180 \mathrm{~m}$ distance in EW and a 1580m distance in NS directions with 15x exaggeration in the vertical direction.

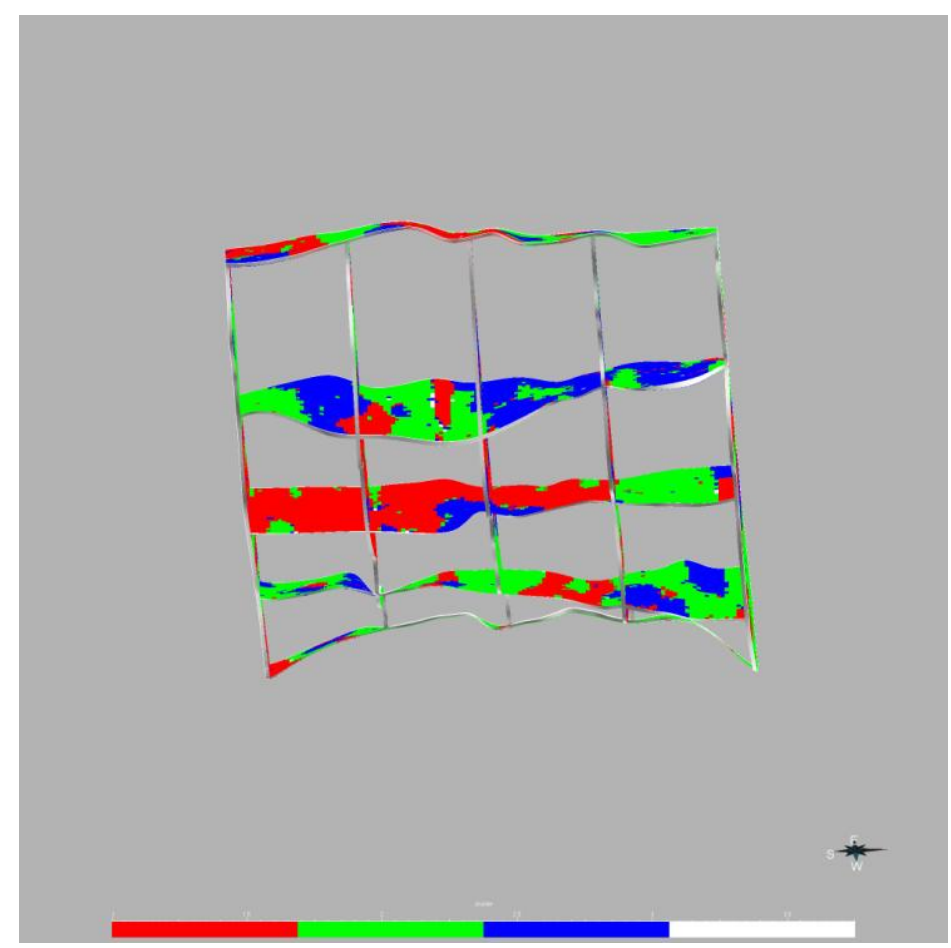

Figure 5.32 A perspective fence section bottom side view along the NS direction of the realization 12, generated by the TSIM with two post-quenching steps simulation method. The vertical slice 2 has been depicted. The model represents a $2180 \mathrm{~m}$ distance in $\mathrm{EW}$ and a $1580 \mathrm{~m}$ distance in NS directions with $15 x$ exaggeration in the vertical direction. 


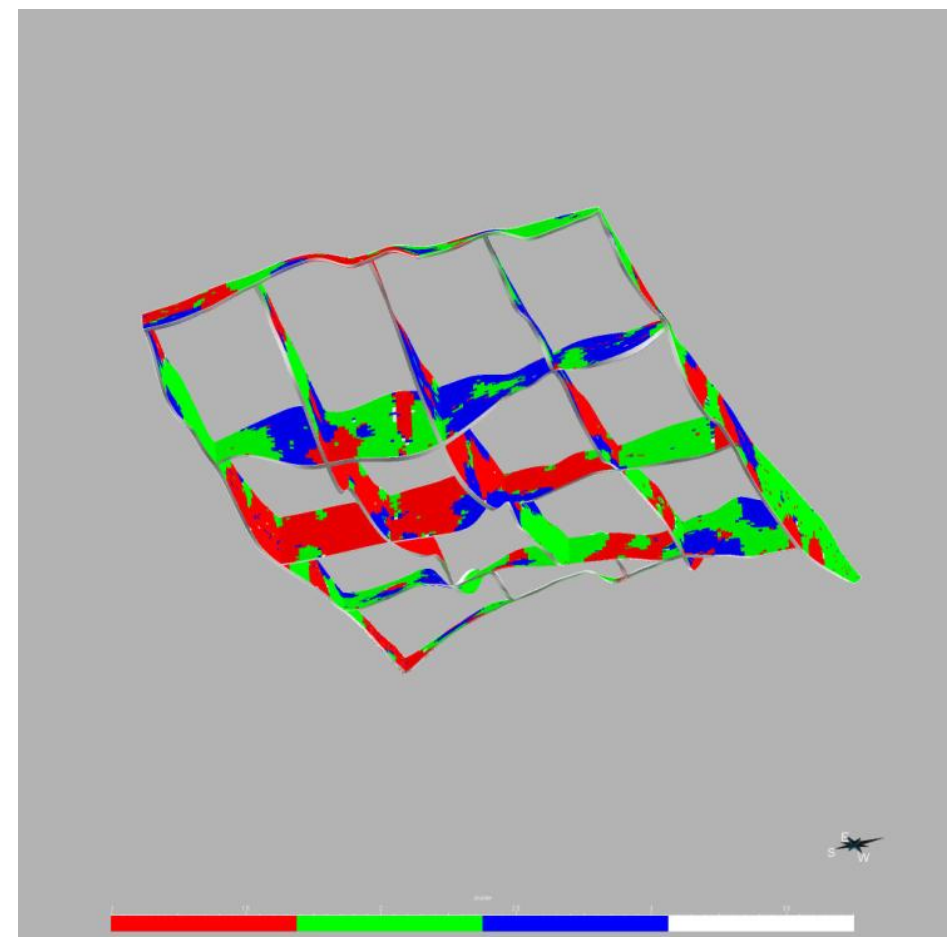

Figure 5.33 Another perspective fence section bottom view along the NS direction of the realization 12, generated by the TSIM with two post-quenching steps simulation method. The vertical slice 2 has been depicted. The model represents a $2180 \mathrm{~m}$ distance in $\mathrm{EW}$ and a $1580 \mathrm{~m}$ distance in NS directions with $15 x$ exaggeration in the vertical direction.

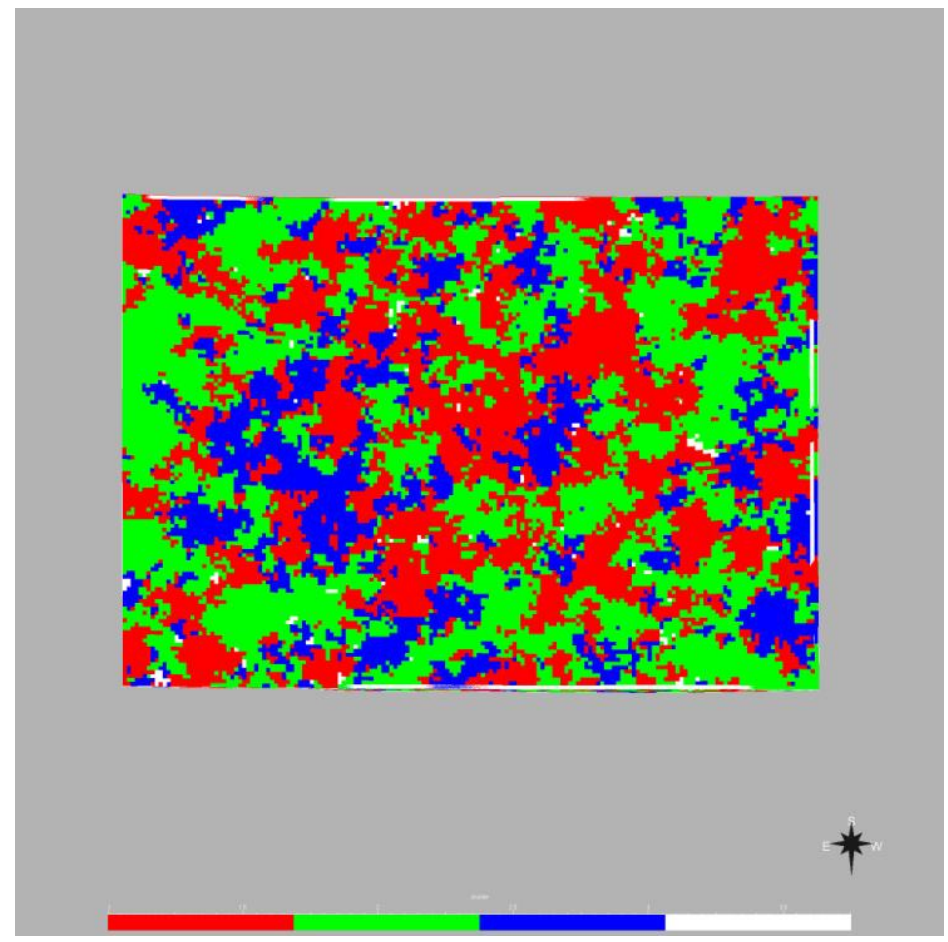

Figure 5.34 A top view of the realization 16, generated by the TSIM without any quenching step simulation method. The model represents a $2180 \mathrm{~m}$ distance in $\mathrm{EW}$ and a $1580 \mathrm{~m}$ distance in NS directions. 


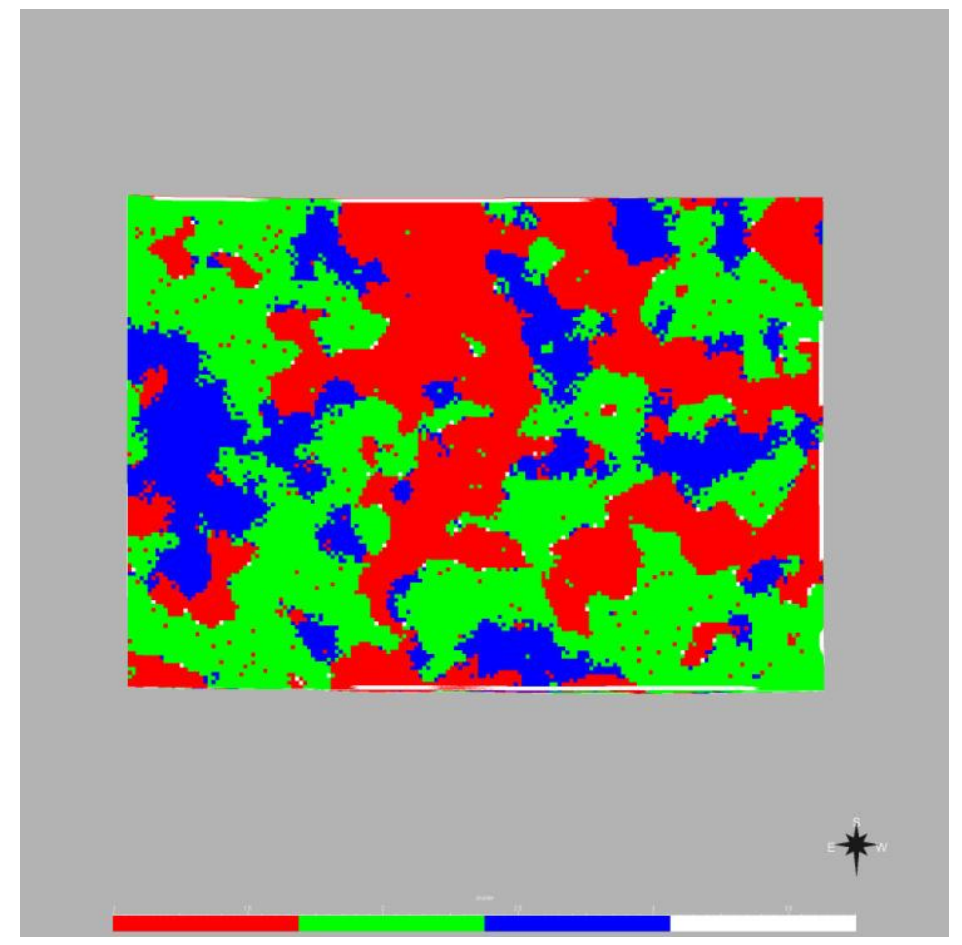

Figure 5.35 A top view of the realization 19, generated by the TSIM with a quenching step simulation method. The model represents a $2180 \mathrm{~m}$ distance in $\mathrm{EW}$ and a $1580 \mathrm{~m}$ distance in NS directions.

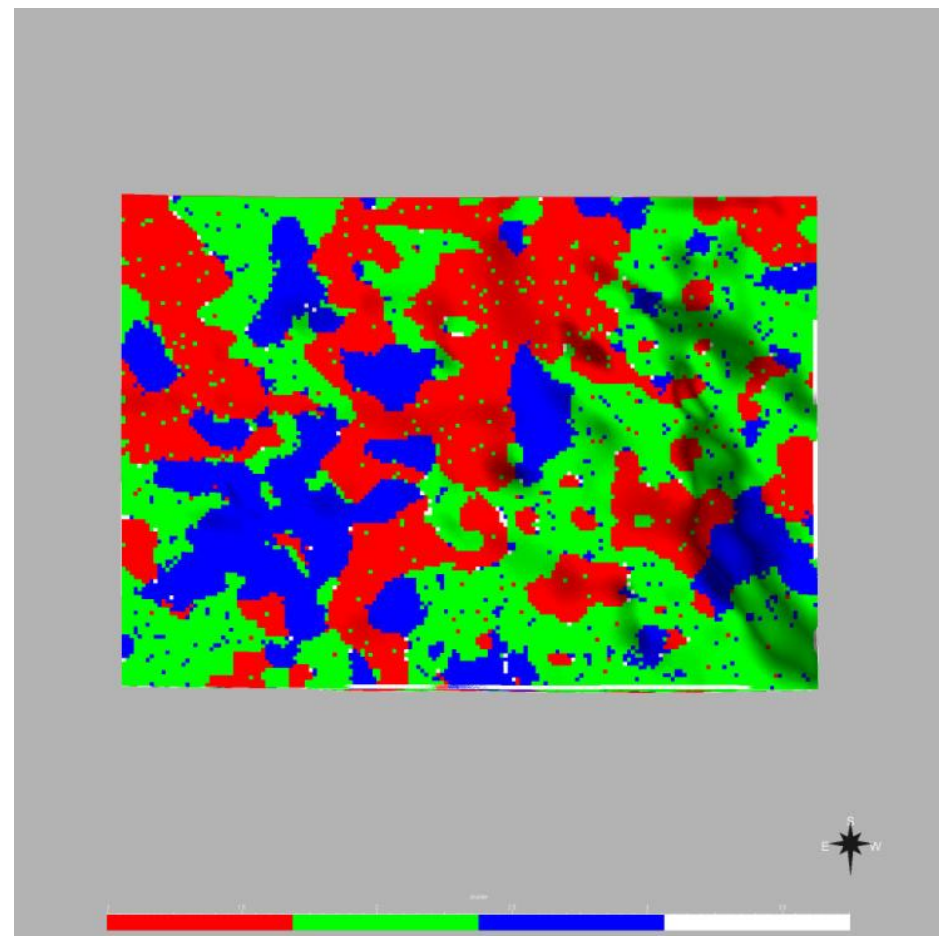

Figure 5.36 A top view of the realization 12, generated by the TSIM with two quenching step simulation method. The model represents a $2180 \mathrm{~m}$ distance in $\mathrm{EW}$ and a $1580 \mathrm{~m}$ distance in NS directions. 


\subsection{Choosing the best realizations}

\subsubsection{Overview:}

One of the most essential steps in analyzing the produced geostatistical analyses is to select a set of produced realizations which conforms the best to the expected characteristics. To choose the best realizations of a simulation method, a stepwise approach has been suggested here. The reasons behind adopting such a stepwise method can be summarized as following:

(1) Reducing the computational difficulties: The computation and verification of all statistics and objective criteria (such as geology, etc.) for all of the realizations would be remarkably demanding. Therefore, it seems more reasonable and practical to limit the number of investigated realizations as much as possible, to make the evaluations more feasible.

(2) Enhancing the fulfillment chance of the other necessary criteria: It seems that if some of the criterions such as proportions-reproduction are met, it can enhance the chance of the fulfillment of the other criterions as well. For example, in a simulation that the proportions of the categories are reproduced better, the chance of a better variograms-reproduction also would expectedly be improved. This suggested idea was evaluated here with the variograms-reproduction test of the best and worst proportions-reproducing realizations of the SISIM geostatistical simulation method (Figure 4.2). A similar comparison was also made about the TPs-reproduction test of the best and worst proportions-reproducing realizations of the SISIM method and as it is obvious from Figure 4.4 and Figure 4.5 , the general result is alike; the best proportions-reproducing realization produces the TPs much better. Fortunately, these results confirmed that a realization of the SISIM method with a better proportions-reproduction gives also in average a better variograms- and TPs-reproduction. One of the reasons for a better variograms-reproduction in a better proportions-reproducing simulation is that, in the indicator variograms, the simulation variogram sill is a function of the proportion of the corresponding category, i.e., $c=p(1-p)$ (Jones and Yuan 2001). It is also known that, the sill of $t_{m k}(h)$ approaches to $p_{k}$, the proportion of 
category $k$, whether $m=k$ or $m \neq k$ (Carle and Fogg 1996, p. 457). Moreover, it seems that, there could be a relation between the proportion of a category and its mean length suggesting that the (absolute value of the) slope near the origin of the auto-transition-probability of a soil class and its proportion should probably have an inverse relation. This inference was made based on the Equation (5-17) and the implication of the existence of a relation between the proportion and mean length of a soil category. The later claim infers that the larger the mean length of a soil category in various directions, the larger the proportion of this category: It could be suggested that the bigger proportion of a category also would lead to a larger mean length. Since the slopes near the origin of the auto-transition-probabilities are inversely related to mean lengths of the categories (according to the Equation (5-17)), this slope should also, more or less is, inversely linked to the relevant proportions. Therefore, it could be suggested that the near origin slopes should be roughly larger in auto-transition-probabilities of the soil classes with smaller proportions, and vice versa. For instance, the soil class 4 with the lowest proportion should represent the largest auto-transition-probably slope near the origin. In other words, near-origin auto-transition-probability of the soil class 4 should roughly demonstrate the largest while the auto-transition-probability of the soil class 2 should have approximately the least slopes among those of all the soil classes. According to Figure 5.3 and

(3) Figure 5.4, the existence of this behavior in the transition-probability models is more or less confirmed for the soil classes in horizontal and vertical directions. Although for soil classes 1 and 2 which had rather similar proportions, these slopes near the origin (and their corresponding transition rates, i.e. -0.0074 vs. 0.0086 in horizontal directions, and -0.17087 vs. -0.17087 in vertical direction) were rather equal or even slightly larger for soil class 2 . Nevertheless, they were not dramatically unlike. This slight deviation form the mentioned expectation could be due to the adjustment of the Markov chain models to fit the necessary statistical constraints during their modeling. In addition, other spatial variability factors might affect the transition-probabilities and their corresponding models.

As a result, it can be suggested that the evaluations of the realizations could start from the proportions-reproduction test as a key initial step and followed by testing the other criteria 
in the next phases. Because, it was suggested that with a better proportions-reproduction of a realization, the sills of the transition-probabilities and variograms as well as the rough near the origin slopes of the auto-transition-probabilities could also be reproduced better. Hence, the selection of better proportions-reproducing realizations would be an important progress in screening realizations with better fulfillment of spatial variability structure reproduction and the other minimum required acceptance criteria.

\subsection{Some attempts to improve the TSIM algorithm}

The results of modeling categorical parameters in the present study suggest a noticeably better performance of the TSIM methods in regenerating the complex geological features in geostatistical simulations especially over the SISIM method. As discussed before, the qualities of the mentioned simulations were assessed based on a number of statistical and geological criterions. Although the efficiency of the TSIM methods was satisfactory, they demanded a considerable computational cost including a long computational time and more complicated models. On the one hand, performing the post-quenching steps on the realizations of the SISIM method, as another alternative, was much quicker than conducting post-quenching steps on the TSIM simulations without quenching steps. Yet, the qualities of final results produced from the SISIM method were not comparable to those of the TSIM methods which produced much better results.

Therefore, an idea was to keep the mentioned advantages of the TSIM method(s) while reducing the algorithm running-time and computational demand of the TSIM algorithm in addition to increasing the capability of integrating more information in the model. To reach this goal of time and computational efficiency as well as a better data integration capability, several attempts were made in the framework of this thesis. Unfortunately, the mentioned attempts have not totally been accomplished and completely succeeded yet. Although they did not reach a deadlock and represented some promising signs, further attempts and several ideas could still be conducted and tested to improve the TSIM algorithm to enhance the qualities and performance of the algorithm.

In all of the proposed corrections for the geostatistical simulation algorithms, the key idea was to modify and update the conditional density function of the local probabilities during the simulation stages. 
Among the examined methods for improving the results were the Bayesian updating to integrate the expert's knowledge or secondary data. As an example, the variations of the likelihood of the categories occurrence in different zones were considered as complementary information to improve the estimation models. However, no significant improvement was detected in the results of this case. Perhaps, this outcome was due to the nature of the data while performing further tests on other data-sets could show clearer distinctions in the performance of the different algorithms.

Another idea was to imitate the simulated annealing procedure (to make the simulated results and the target closer to each other) in terms of the transition-probabilities, just in the newer approach, during the simulation algorithm rather than after that. The expected advantages were:

(1) Reducing the computational time.

(2) Further improvements in the final models because of forcing the algorithm to produce sensible patterns from the beginning rather than modifying the produced patterns after their generations.

In this framework, the local CCDFs for the geostatistical simulations had to be updated during the simulation course. Some attempts have been reported in the literature for updating the CCDFs during or after the simulation course based on the proportions of the facies to avoid a notable departure from the expected ones for instance about the SISIM method (Scares 1998; Journel and Xu 1994; Goovaerts 1996. The simulation methods like indicator simulation methods that do not rely on the transformation and backtransformation of the distribution of data and results do not also reproduce the priori cumulative distribution function. Hence, the categories distribution function (proportions) of the realizations generated by these methods usually deviate considerably from the expected distributions. Several methods have been proposed to force the realizations of these methods to reproduce the expected proportions (Scares 1998; Journel and Xu 1994; Goovaerts 1996. Although, the only concern was not to reproduce the expected proportions, a similar idea could be applied to improve the TSIM method based on the deviations of the produced proportions from their expected values so that the transitionprobabilities also could be reproduced acceptably. Nevertheless, for new proposed framework this correction would be conducted considering the deviations of the local CCDFs in the framework of the transition-probabilities conditioned to the nearest points 
to the simulation grid node and the deviation of the observed transition-probabilities in the simulation from those of the (Markov chain) models.

To conduct these corrections in the TSIM algorithm, some modifications were made in the TSIM Fortran program to estimate the global transition-probabilities of each realization at each simulation step and update the local conditional density functions during the simulation run. However, several simplifications were considered in this idea to make the changes more practical. Some of the mentioned simplifications included;

(a) The transition-probabilities were computed for a simple specific neighborhood of the simulation point with averaging the distance and transition-probabilities inside the neighborhood. The neighborhood was defined by some vectors from the simulation point. For instance, a set of vectors like $(0,0,+1),(0,+1,0),(0,0,-1),(0,-$ $1,0)$, etc. were defined. In this case, for example, the vector $(0,+1,0)$ shows a simulation node in the neighborhood which is located in only one node distance of $\mathrm{y}$ direction from the simulation point. The program was using these vectors to identify the neighborhood in which it was searching for simulated or data points to calculate the transition-probabilities.

(b) The model transition-probabilities were estimated using the corresponding Markov chain model of the transition-probabilities for a single short lag.

(c) A simple corrector for updating the local CCDFs were considered during the simulation proportional to the extent of the deviation of the calculated overall TPs till that stage of the simulation from the model TPs. Therefore, this corrector was adding a value to the local proportion of a category when it became less than the expected proportion calculated to the previous simulation stages and subtracting a value in the contrary case. An important point is that, the local CCDFs were conditioned to the neighboring data or simulated points. This corrector in general was defined as a vector that were added to or subtracted from the proportions vector calculated as the local CCDF in a simulation stage. For example, if there are three points in the neighborhood, with the categories 2 in two of them and category 1 in the other one then it should be calculated that what are the probabilities of transition from classes 2 and 1 to each of the other possible categories in average? This calculation were conducted based on the existing simulated and sample points till that stage of the simulation and what the difference of them to the expected probabilities was. 
In spite of some achieved improvements in the results, artifacts also were seen and in general, the achieved advantages were not that sufficient so far to present the proposed method as a sufficient enhancement in the TSIM method while it needs further development and adjustments. The reason could be the existence of several simplifications.

The mentioned improvements were evaluated from two points of view; how quicker the program in a specific condition (i.e. specific data-set and parameters) did work, and how better the produced simulation patterns were. Therefore, if the new algorithm could produce at least a comparable simulation patterns to those produced by the TSIM method with some quenching steps in a shorter time, it would be concluded that the proposed method have functioned well. However, in spite of quicker run-time, the produced patterns from this algorithm were not satisfactory-enough yet. Although the logic of the corrections seems reasonable, the applied simplifications and some other points in the algorithm could affect the overall results and reduce their qualities.

Another proposed approach was to imitate the multiple-grid simulation strategy in which the quenching was started from a coarser simulation-grid before going to the finer ones that could probably reduce the quenching time and increase its efficiency due to a less need to the pixels-swapping during the quenching steps. Still, a considerable improvement was not seen yet, especially in terms of the computational time though the proportion reproduction became better. The simplified test here was by performing the simulations stage-by-stage from the coarser grids and feeding the results as conditioning data to the next finer-grid stage. However, it is expected that by employing more proper programming techniques, the algorithm could become quicker and more efficient.

In addition, there were also some unanswered questions such as "should any selection be done in each simulation stage to feed the selected realization into the next simulation stage or not?" Some cases have been tested to answer this question but additional assessments are required to be accomplished yet to reach a reasonable answer.

Because the desirable goal was not fully achieved so far in this investigation and more attempts are necessary to reach the expected point, the more details were not explained here. 


\subsection{Closing remarks for chapter 5 :}

The suggested steps to assess of the minimum acceptance statistical criteria for the geostatistical simulations in the present study can be summarized as following:

1. Evaluation of the input data reproduction as the initial condition which should be fulfilled for all the realizations, with some fluctuations.

2. The proportions-reproduction should be checked in the next step, from which a less number of realizations will be suggested for the evaluations in the next stages. The selected realizations from this step are expected to represent a better variograms-reproduction according to the discussions in the previous sections.

3. The spatial variability reproductions including the variograms- and transition-probabilities-reproductions should be assessed next. The examples and logical reasoning suggested that each of the variograms and transition-probabilities convey a side of the spatial variability structure. Therefore, their simultaneous application can improve the understanding and verification level of the spatial variability reproduction for the realizations which is suggested for similar cases.

The mentioned steps include solely the statistical checks of the realizations. However, the geological confirmation of the realizations should be considered as another important criterion. However, as explained before, due to the difficulty of the one-by-one geological check of all the realizations, the assessments for geological soundness were conducted on the selected statistically sound realizations.

At the end of this study, since the selected realizations in terms of proportionsreproduction fulfilled the other criteria as well, they were selected as the best realizations. It was also suggested that this stepwise criterion could be applied for other similar problems too. 


\section{Comparison of different geostatistical simulation methods based on their results}

\subsection{Overview}

In addition to the selection of the best realizations and checking their acceptability and quality, a question may arise that which simulation method (algorithm) in general worked better and what were the pros and cons of each one?

To answer this question appropriately, the following three factors were considered in this study:

(1) Geostatistical and statistical factors.

(2) Geological considerations.

(3) Computational considerations.

Following, more details about each factor have been discussed.

\subsection{Evaluations of the geostatistical simulation methods}

\subsubsection{Based on the (geo-) statistical factors}

These assessments included evaluating how good the realizations of each simulation method (algorithm), in general, met the statistical and geostatistical requirements. In other words, the aim was to evaluate how close the statistics and geostatistics of the results for each simulation algorithm to the expected quantities or qualities were, considering a number of statistical measures. A simulation method or algorithm which either can generate a more number of realizations with acceptable statistical and geostatistical factors or closer statistics and geostatistics to the target would be regarded as a better simulation approach. To conduct such evaluations, one might consider the proposed criterions by Oy Leuangthong et al. 2004 like minimum conditions to regard a realization as an acceptable one but this time to compare the efficiencies of different simulation methods. Yet, in the initial proposed scheme, the attention was drawn to the minimum conditions to validate the realizations of a simulation algorithm rather than evaluating the simulation algorithm itself. It seems that, evaluating and comparing the same criterions 
among a set of the results from different simulation methods could provide a framework to compare different simulation methods. However, these criterions were expressed more qualitatively than quantitatively in the initial framework. Ortiz and Leuangthong (2007) have introduced a quantitative statistical scheme to validate the variogram-reproduction of Gaussian simulations using some statistics like $\mathrm{T}^{2}$-Hotelling's test (an extension of Student's T-test). However, the verification of the multivariate Gaussian behavior of the SISIM and TSIM methods was tremendously demanding due to their extremely complex natures for such indicator data $(\mathrm{I}=1$ or 0$)$ or their finally inferred categories (i.e. soil categories here). Actually, Emery's method is more suited for continuous data with a Gaussian behavior. Emery (2008) also suggested an analytical method to validate the geostatistical simulation algorithms based on some statistical tests to evaluate the agreement between the experimental statistics (such as means, variograms, and indicator variograms, calculated for several realizations) of the simulated random field and corresponding model statistics with a statistical acceptance tolerance. Obviously, the existing fluctuations in the experimental statistics of the geostatistical simulations should be considered. He discussed the basic assumptions, relevant statistical relations, his proposed statistical tests, and how to apply the mentioned statistical measures and checks. Relevant computer codes have also been provided by him. However, in this study, the variograms-reproduction checks were conducted qualitatively and considering the relevant plots. Yet, a brief description about the Xavier Emery's quantitative method, its basic assumptions, and why it has not been applied here has been discussed very briefly in the coming parts.

Given that the statistics of the finite simulated fields and the model have been calculated, some fluctuations among the statistics of the realizations as well as some departures from the model should naturally be observed. Emery's approach basically determines whether or not the agreement between the simulations and model statistics considering the observed fluctuations among them can be rejected in an acceptance tolerance? This tolerance can be expressed in terms of some statistical concepts such as the significance or confidence levels. However, in the case that the accordance of these two sets of statistics (from model and from simulation results) is not rejectable, the suitability of a simulation algorithm can not necessarily be admitted. In Emery's method (Emery 2008), the mean and dispersion variance of the simulated random variable in the generated realizations are tested using the T-student test. In this study, however, the application of the chi-square together with the deviation rates framework (the latter suggested by the 
author) is proposed to validate and compare the categorical parameters histogramreproduction of different simulation methods. Another statistical test that Emery suggested to compare the algorithms was the variogram-reproduction in single or multiple lags. The $\mathrm{T}$ - and $\mathrm{T}^{2}$ - tests are applied in his scheme (Emery 2008) to check the existence of a bias in the model variogram reproduction and compare the simulation algorithms so that one can determine the confidence-interval with a pre-specified probability of $(1-\alpha)$. However, this could not provide a clear criterion to compare the algorithms. In addition, this confidence-interval will be a function of the standard deviation of the simulation variograms and the number of realizations. Having a glance at the calculated variograms of the SISIM simulations for the number of 20 and 100 realizations, respectively (Figure 6.1 and Figure 6.2 ), it can be suggested (qualitatively) that the standard deviation of the variograms of the 20 and 100 realizations should approximately be similar because the dispersions of the two mentioned series are very similar according to the related variogram graphs. On the other hand, the numbers of the realizations are considerably different and the term $\sqrt{N-1}$ in the corresponding formula, i.e.

$$
\begin{gathered}
\left.\Gamma_{D}(h)=\frac{1}{2 K_{h}(0)} \int_{D \cap D_{-h}} \mathbb{I}(x+h)-Y(x)^{-2}\right] d x, \\
\frac{\bar{\Gamma}_{D}(h)-\gamma(h)}{S_{\Gamma_{D}}(h)} \sqrt{N-1} \sim T_{N-1}
\end{gathered}
$$

will notably be different among 20 and 100 realizations $\left(\sqrt{N_{1}-1}=4.358\right.$ with $\mathrm{N}_{1}=20$ is well under the half of the $\sqrt{N_{2}-1}=9.949$ with $\mathrm{N}_{2}=100$ ).

In the above equations (6-1 and 6-2), the regional variogram of the random field $Y$ over domain $D$ with the average of $Y(D)$ for a given lag vector $h$ is $\Gamma_{D}(h), D_{-h}$ stands for the domain $D$ shifted by the vector $-h, K_{h}$ is the geometric covariogram of $D_{h} \cap D_{-h}$ and specifically $K_{h}(0)$ is the measure of $D_{h} \cap D_{-h}$, and finally $S_{\Gamma_{D}(h)}$ is the standard deviation of the regional variogram of the random field (Emery 2008, pp. 1611-1612).

Because the expected variogram values at lag $h$, i.e. $\gamma(h)$ are the same for both cases and the critical t-values, $\mathrm{T}_{\mathrm{N}-1}$, for both sets of realizations are also almost equal (2.093 vs. 1.984), the calculated interval for the variogram average $\bar{\Gamma}_{D}$ will be much wider for the 100-realizations set than that of 20-realizations set. Therefore, in this case, the Emery's method does not help so much in comparing the simulation methods (algorithms). Clearly, it would not provide so much information about the acceptability interval of the 
simulation variograms of each simulation method since it gives two different evaluations of the acceptability for two spectrums of realization variograms. Based on the same framework that Julian Ortiz et al (2007) have discussed, when the calculated values of $t$ (for a single-lag test) or $\mathrm{t}^{2}$ (for a multiple-lag test) exceed their critical values from $\mathrm{T}$ (for single- lag tests) or F (for multiple-lag tests) distributions with the corresponding degrees of freedom (DF) and significance level $(\alpha)$, the hypothesis that the mean vector of realization-variograms (including the mean variogram values in a number of lag distances) is the representative of the input model variogram would be rejected (Emery 2008, pp. 1611-1612). It could be suggested that if the variograms-reproduction of a method is rejected while it can not be rejected for another method, the second method functions better in terms of variogram reproduction. The reason for this deduction is because there is no strong evidence that the variograms-reproduction of the latter method to be rejected with the specified significance level.

The reasons which based on them it was decided not to apply Emery's method here for the comparison of the simulation techniques and adopted another methodology have been summarized as following:

(b) The multi-Gaussian assumption as a perquisite for the Emery's suggested framework could not readily be proved and verified for such a discrete-value simulation scheme. Basically, the Emery's suggested method was suited for continuous attributes and not for the categorical ones.

(c) The method could potentially be applied for variograms or transitionprobabilities reproduction evaluation because the values of variograms or transition probabilities could be seen as random fields. However, it can impose considerable complications and more extra calculations whereas the proposed qualitative comparisons in the present study could be conducted much easier.

Emery's suggested framework does not provide a straightforward platform to compare the variograms-reproduction among different simulation methods. Actually, it can solely test whether or not in a simulation method, the average variogram falls out of an acceptable interval form the model with a specific statistical significance-level? If it falls out of the mentioned acceptance interval, one can reject the variogram reproduction. However, if it does not fall out of the mentioned interval, the variogram reproduction can not be rejected in the relevant simulation method. Therefore, Emery's method does not add so much 
value to the variogram reproduction evaluations. Especially this is because; the mentioned interval depends very much on the standard deviation of the simulation variograms (experimental variograms calculated from the simulation results) and the number of realizations in this method. Therefore, it does not facilitate the comparison of different simulation methods noticeably. For example, in general, the standard deviation of the simulation variograms is considerably higher in SISIM method compared to that of the TSIM method. Therefore, the acceptance interval of the average variogram should be much wider for the SISM than that of the TSIM method considering the same significance-level. However, the higher standard deviation means a higher deviation of the spectrum of simulation variograms from the model. In other words, the acceptable departures of the average and the model variograms are higher in SISIM compared to those of the TSIM method. Nevertheless, the Emery's method indicates that the latter has higher chance to be rejected. Considering the mentioned drawbacks of the method suggested by Emery (2008) and Ortiz et al. (2007) for variogram and other statistics reproduction test, a qualitative scheme was applied in this study to compare the efficiencies of different simulation methods. Though the proposed scheme in the present study has been devised more qualitatively, a development of quantitative criterions could also be suggested.

(d) The general proposed framework here for the comparison of the simulation methods was to follow a stepwise workflow, starting from testing the conditioning to input data, histogram-/proportions-reproductions of the simulations, the variogram and transition-probabilities reproduction, and finally the geological soundness. For variograms and TPs-reproduction test, a qualitative verification and comparison scheme like in the previous section (for selecting the best realizations) was suggested, just this time considering the extent of difference from the model and their goodness among different methods. In this case, the dispersion of variograms among different realizations was considered as a disadvantage because the more simulation variograms dispersion the more realization variograms will depart from the model variograms. With such an insight, the variograms-reproduction for SISIM method was in general weaker compared to TSIM methods, especially for TSIM methods with post-quenching steps. Although, in the vertical variograms of the 
TSIM method without quenching, the difference of the variogram values from the model are more but closer to each other. In other words, in the TSIM method without quenching steps, the simulations have much closer spatial structures together but they are more different from the model in vertical structure comparing to the SISIM method. Nevertheless, adding the quenching steps, improves this difference to the model significantly but the results are even closer to each other. Therefore, considering the proposed criterions, a general judgment could be that the TSIM has been more efficient than SISIM in terms of statistical parameters reproduction, especially when further quenching steps are considered.

As discussed before and according to the mentioned criterions, one can conclude that, in general, the TSIM methods especially with some quenching steps produced much better realizations in terms of geostatistical criterions fulfillment and even in the geological soundness insight. The application of too many quenching steps, although, did not improve the results considerably and can be avoided.

\subsubsection{Evaluations based on geological acceptability:}

As discussed before in Section 5.3.6, the patterns produced by the TSIM method were geologically much sounder than those of the SISIM method.

In addition, there are more possibility of integrating the subjective information and geological interpretations in the TSIM framework.

The details of how this conclusion was made, has been addressed in that Section 5.3.6.

\subsubsection{Evaluations based on the speed of the algorithms and the ease of their applications:}

About the speed of the running any algorithm, the SISIM method was considerably quicker. The ease of applying each method does not seem greatly different. Only in the TSIM method, more care should be drawn to the perquisites of the Markov chain models during the modeling of the transition-probabilities. However, this difficulty does not seem to be a great challenge. In addition, in the case of the absence of abundant observations, 
the TSIM method that can integrate more subjective information and geological interpretations could be applied easier.

The worst drawback of the TSIM algorithm is its considerably longer run-time. For example in the PC system ${ }^{1}$ which has been applied for this research, producing a TSIM realization with two quenching steps for a simulation grid of $175 \times 124 \times 28$ took about 1 hour, 6 minutes, and 7 seconds while as a SISIM realization was produced in about 1 minute, and 20 seconds. Even a TSIM simulation without any quenching steps took about 27 minutes which is still considerably much more than the SISIM simulation time. Although, the computational cost is much higher in the TSIM method, it can be considered an acceptable shortcoming because with the advent of high-speed computers and computational techniques, this cost would become affordable for the benefit of producing better realizations in terms of statistical and geological soundness.

Still, some additional improvements can be proposed to overcome the problem of TSIM being too much time-consuming. A more detailed discussion about the efficiency of TSIM and its comparison to the other geostatistical simulation methods as well as some attempts and suggestions for improving the TSIM method and its efficiency will be given in the coming Sections 5.57.4.

\subsection{A number of practical points on modeling steps in this research}

The application of the Wingslib software as a MS Windows interface between the user and the GSLIB programs, not only facilitates the use of those programs but it also provides a framework to combine or execute them and show the results for a considerably big numbers of times and in each favorable orders by its Batch script window, run the external programs, and store the $\log$ window to evaluate the execution details of each program and analysis.

\footnotetext{
${ }^{1}$ Intel(R) Pentium(R) 4 CPU, 3.2 GHz, 3.2 GHz, 2.00 GB of RAM, Microsoft Windows XP, Professional x64 Edition Version 2003, Service Pack 2.
} 

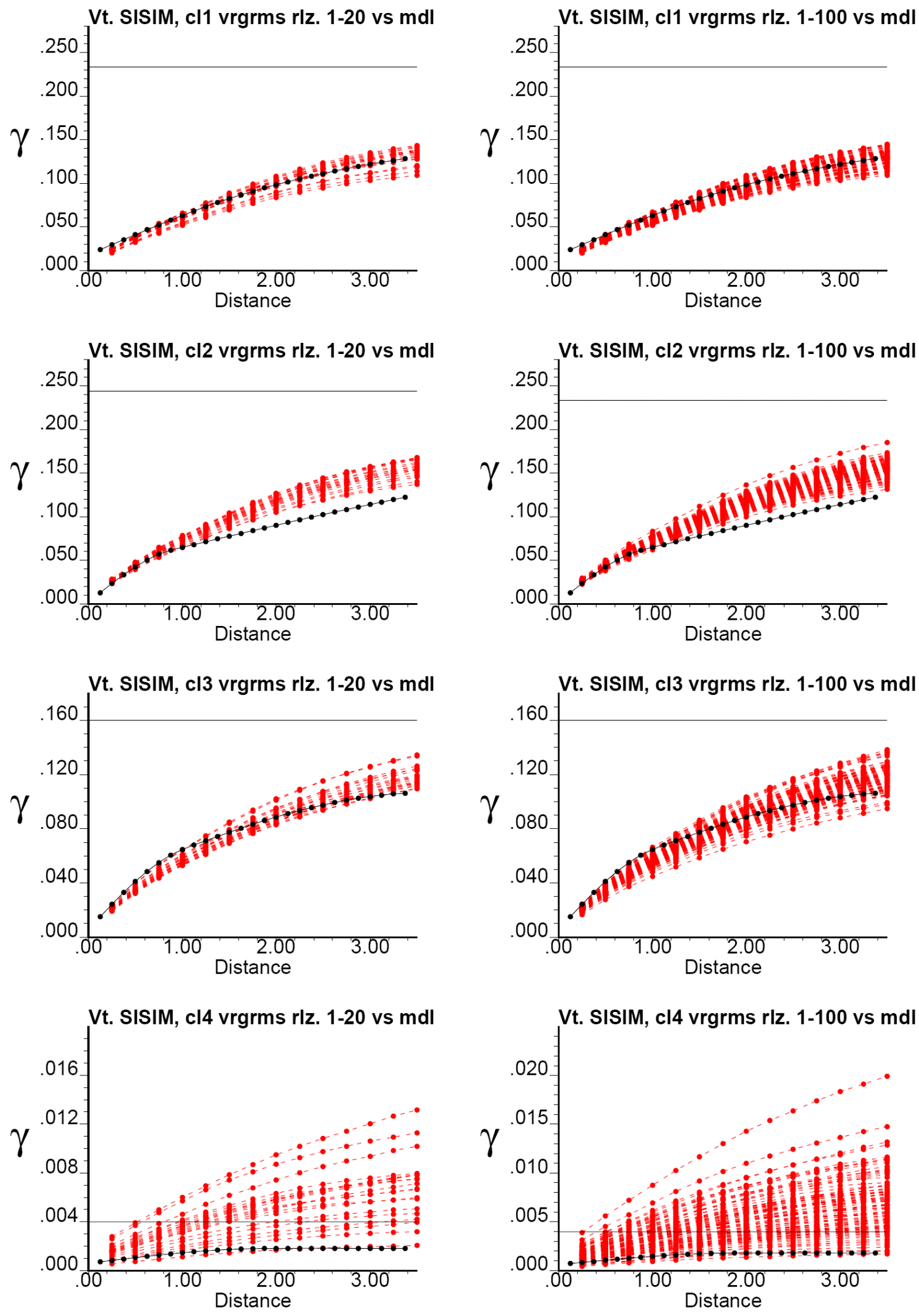

Figure 6.1 Vertical indicator variograms of the generated realizations by the SISIM method of the 20-realization run (left-side red lines) and 100-realization run (right side red lines) and their corresponding models (black lines). 

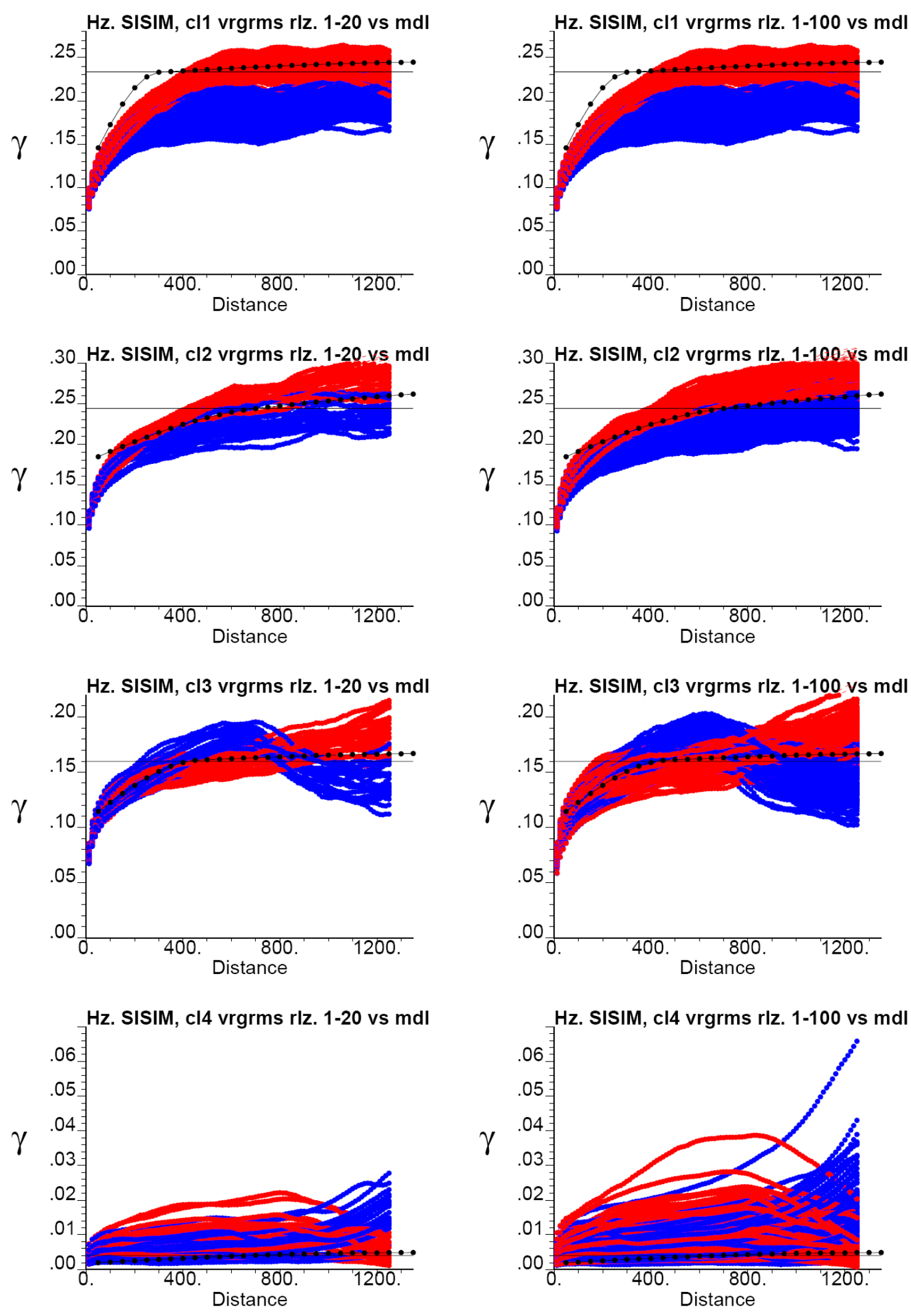

Figure 6.2 Horizontal indicator variograms of the generated realizations by the SISIM method of the 20-realization run (left-side red lines) and 100-realization run (right side red lines) and their corresponding models (black lines). 
As it is clear in this dissertation, there were some steps in which the calculation of a parameter such as indicator variogram of the realizations has been performed for a big number of times. To be able to do so, the Batch script window of the Wingslib software was applied. For this purpose, first, the required parameter files including parameters which had to be changed (e.g. the realization number) had to be created and stored with different names. In this research, the MS Excel pages with some functions and macros were employed to create a big file including the parameter files parameter units. The Replace pioneer 2.63 software was applied to split the units of parameters so that each unit as a representative of a parameter file contained the required parameters for that round of calculation. Each file was saved with a different name in which there was a base name ending with a different number, for example; "GR-vt_tsim...1.par", "GRvt_tsim...2.par", GR-vt_tsim...N.par". All the produced parameter files were imported into the Wingslib atmosphere (added to the program trees) by the Import all parameter files option in File menu of the main Wingslib toolbar. To call the created programs for running or viewing, the best way would be to create a Batch script file (with *.wgb extension) including the name of the mentioned parameter files which can be created again in a MS Excel sheet where the name of the parameter files can be changed by adding a number to the base name of the parameter file. The general format of Batch script file line stars with Run or View according to the aim of user either to run a program or show its results. The following line is an example of such a line in a Batch script line:

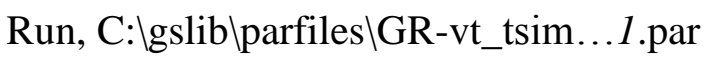

Run, C:Igsliblparfiles\GR-vt_tsim...2.par

....

$\cdots$

Run, C:|gsliblparfiles\GR-vt_tsim...N.par

For managing the big ASCII files, the Textpad software was applied. For instance, since the calculation of the frequency of the soil categories in the produced realizations is very time-consuming for the Wingslib, the output ASCII file lines including the category codes (e.g. 1, 2, 3, 4) were found, marked, selected, and copied into another empty page. The number of lines including this category could be read in the Textpad software to find the frequencies of the categories in a specific realization or even in all the realizations.

Another practical point is related to the postscript files produced by Wingslib as the graphical outputs of the programs. Since the graphical and text options of the software is 
very limited for making a suitable favorite graphical output, an applied solution in this research was to edit the *.ps or *eps files by a normal text editor such as Textpad. The Textpad software was especially very suitable because of its capabilities to work with big ASCII files and other capabilities such as producing macros. 


\section{Summary and conclusions}

\subsection{Synopsis}

A high-resolution and accurate subsurface model which integrates a wide range of information from various sources such as samples and observations, expert's and priori knowledge, prediction or simulation results, etc., is an essential tool in evaluations of numerous practical problems such as mining and mineral resources, hydrocarbon or geothermal reservoirs, hydrogeology, and so on. More specifically, having a precise subsoil and subsurface stratification, existing material types, and underlying geological characteristics is of vital importance for the geotechnical engineering applications (e.g. Farshbaf Aghajani and Soltani-Jigheh 2009, p. 347). However, the limited and at times indirect accesses to the subsurface as well as the high heterogeneities and complexities in subsurface, make the modeling practice even more challenging.

In such applications, the aim would be to construct the complex subsurface model(s) thoroughly and precisely, having a limited number of samples usually distributed irregularly over the study area. This model(s) should reflect and imitate the underlying real characteristics. With such a limited data sources, geostatistical methods offer appropriate alternatives to establish an accurate model while increasing the capability of combining more information as well as providing the uncertainty measures of the modeling.

In the conducted project, the aim was to construct a three-dimensional model of the subsurface in a test site of the Göttingen area to evaluate the capabilities of geostatistical methods to model, estimate, and simulate the facies, or more specifically here, unconsolidated sediment types, using geostatistical and stochastic methods. The modeled parameters were geotechnical categories of the subsurface unconsolidated materials form the Quaternary here. In general, modeling such categorical parameters, like the facies types, is usually the initial step to construct the continuous variable models such as the models of permeability, hydraulic conductivity, and so on (Deutsch 2002, pp. 21-25). Due to the improved and proper results obtained in this study, the explained steps for making such a model can be suggested in constructing the similar models for similar conditions. In the Göttingen project, a wide range of data which had been collected from previous studies and ongoing measurements (at the time of the start of this research) such as several drillings were used as the basis for constructing the expected model. The 
classification of the taken samples had been conducted according to a framework based on German geothechnical standard of DIN 18196 to obtain the necessary data for subsurface modeling in the department of Applied Geology of the University of Göttingen (Dr. Bianca Wagner, Dr. Torsten Lange, and other project coworkers).

To achieve the sample data in vertical direction, the data were taken vertically in equidistance spacing along the sampling drillings of the test site. In pixel-based modeling of facies, the first step after collecting the available data is to assign the indicator values and create an indicator field for each soil or facies category. This indicator value equals to $\underline{1}$ at the locations where the relevant category exists and $\underline{0}$ otherwise. These indicator values are applied as the basis to the geostatistical estimation and simulation of the existing soil categories.

Similar to what has been conducted in the present study, the estimation/simulation grid space should be transformed into a new one which better includes the stationarity of estimation/simulation space according to the stratigraphic structure and genesis of the layers. The transformed grid system chosen in this study was proportional to the top and bottom surfaces of the target layer due to the deformation of the layers during the sedimentation and the lack of significant erosion. It should be reminded that the quite homogeneous and sometimes containing artificial fillings uppermost layer was excluded in this model and has been modeled separately in order to improve the stationarity conditions inside the study zone and take the geological knowledge into account by delineating the geologically more similar units in separate modeling zones. Hence, the hill sides (which include the solifluction materials) as well as the deep holes in the modeling area were not included in this model and have been modeled separately.

In the original space, as it was expected for the target layer, strong correlations were detected between the elevations of top and bottom surfaces and a strong negative correlation between the bottom surface elevation and the thickness. This has represented that, the top and bottom surfaces of the target layer are correspondent to each other while the layer is thicker wherever the bottom surface is in a lower elevation.

In the transformed-grid space, the stationarity assumption was adopted since the soil categories were distributed almost homogenously over different parts of the model. The scatter-plots of the soil categories against the distance-coordinates and indicator variograms of soil categories in the transformed space were employed to evaluate and confirm the existence of stationarity conditions. In these scatter-plots and indicator variograms, there were no evidences of significant detectable trends. The evaluation of 
the variograms to assess the existence of probable trends is a common solution to detect notable available trends.

Then the spatial structures of the data variability were calculated and modeled for the directional indicator variograms of each soil category. The variogram models validation and then the indicator kriging were the next steps.

Indicator variogram models and estimation models were validated and some trial-anderror adjustments in the models as well as the estimation parameters were applied based on the two methods of cross-validation and jackknifing. The cross-validation and doing jackknife of the models of categorical attributes requires special techniques which have been discussed in section 3.2.3 .

The cross-validation and jackknifing of the models and re-plotting the variograms with a less number of boreholes here (e.g.

Figure 3.5) suggested that the selected models were not only acceptable-enough but still a less number of drillings (even one-half of drillings for a similar area) could be applied in comparable cases to achieve more or less similar accuracies.

The indicator kriging (IK) can be performed for the indicator variables to produce the probability of the existence of each soil category in each estimation point. However, the favorite outcome would be a three-dimensional map of the predicted soil facies. To do so, the soil classes with the highest estimated probability of occurrence (from indicator kriging) could be assigned to each estimation point of the model. The ik3d or kt $3 \mathrm{~d}$ programs of the GSLIB (or Wingslib) software can be employed to calculate the probability of the occurrence of each soil class at each estimation location. The first program (ik3d) calculates these probabilities, and performs the order-relation violation corrections all at one run and hence is much simpler in application. However, it does not let the user to consider different anisotropy or estimation parameters for various soil classes. The application of the second program (kt3d) makes this possible but with extra steps to conduct the order-relation violation corrections and perform the IK separately for each soil category which would be dramatically time-consuming and challenging. In addition, the estimation variances as a measure of estimation uncertainty can be obtained in the second method. In this study, however, in spite of testing both methods, the ultimate decision was to choose the first method and use its results for producing the final outcomes of the IK estimations. In the next step, a MATLAB code, written by the author of this dissertation, was employed to assign the most probable soil class (according to the 
IK probability estimations) to each estimation point. The final three-dimensional model was then created after back-transforming the estimation grid into the original system.

The indicator kriging method is not capable of reproducing the expected features and geology so effectively since it contains the smoothing problem. In other words, the subtle variations and global features could not be reflected in the generated models. Actually, kriging produces patterns in the model that do not imitate the geological realities so adequately. In other words, kriging is globally inaccurate, e.g. generally it does not reproduce the expected global histogram. In other words, kriging can produce maps with minimum estimation error while its produced maps could not reproduce the extremes and global features. These subtle variations can become particularly essential in some applications such as modeling the fluid flows in the porous media. The only point here is that this discussion is about the indicator fields of the estimations using IK. Nevertheless, the behavior of final estimated categories by the mentioned scheme of the most probable soil class at each estimation point could be more different and complicated to easily be predicted. Still, the global-statistics-reproduction would not necessarily be fulfilled in IK final estimations of the soil classes or any categorical attribute (such as lithofacies and so on). Figure 3.3 and Figure 3.4 represent this fact that the IK estimation of soil classes does not reproduce the expected global histogram and indicator variograms so adequately. However the results of the cross-validation analyses confirms its being locally accurate (see parts (a) and (b) from Section 3.2.3).

Geostatistical simulation methods provide a set of solutions to overcome the aforementioned problem of the kriging. Therefore, they can produce the patterns which better resemble the reality of the subsurface by putting the minimum squared error condition aside to make them capable of reproducing the fine variations and global statistics more adequately (Hohn 1999, p. 180).

The sequential indicator simulation (SISIM) and the transition probability Markov chain (TP/MC) methods as solutions for reproducing the local fine variations and global geological features were applied, assessed, and compared to each other, in this study.

The final geostatistical simulation outcomes and assessments of their methods have suggested the application of TP/MC method as a more suitable solution in simulating the subsurface categorical parameters over a number of other methods in sedimentary environments.

The quality of simulations was then assessed, the best realizations were suggested for further evaluations, and the simulation methods were compared. 


\subsection{Evaluations of the geostatistical realizations and simulation methods}

To evaluate the quality of generated geostatistical realizations and decide on their acceptability, several statistical and geostatistical as well as geological criterions were assessed. Evaluated statistical/geostatistical criterions included conditioning of the simulations to the input data, along with the histogram-, indicator variograms-, and transition-probabilities-reproduction of the simulations. Though, the assessment of geological soundness was a more challenging question due to its more interpretive and subjective than quantitative nature, or at least the lack of a general quantitative framework for such evaluations. In addition, since the geotechnical classes considered in this study did not directly correspond to the geological categories, although these categories (that were present in this test site) conveyed some geological meanings such as their grain-size composition or the sediment types, the evaluation of the geological soundness for these simulations was not so an easy and straightforward task. Although in the TP/MC framework, one can integrate an extent of geological information such as the length statistics of the layers and juxtapositioning tendencies as well. Some additional concepts were also applied in this study to evaluate the soundness of the generated geological simulations. Among these complementary concepts for geological evaluations of the quality of simulations, the coarsening upward or downward patterns, and the connectivity and layering of the soil categories were also considered in this study. Moreover, the Walther's law of facies succession was deemed to infer the soil classes which probably can occur beside each other bearing in mind the vertical successions observed in the boreholes (see Section 5.3.6). The preference of applying the vertical successions observed in the boreholes was because of the abundant and more reliable information with much higher density of the samples along the boreholes (in the vertical direction). From these geological acceptability evaluations it could not be a big contradiction to the expectations found. The employed framework to evaluate the geological soundness of models for this study can be applied for the similar cases while the development of a more general quantitative scheme could be proposed.

The test of conditioning to the input data was simply done using a MATLAB code, written by the author of this thesis for such purposes. This program finds the nearest 
simulation or estimation grid nodes to the sample points, compares there quantities with the observation, and finally calculates the ratio (or percentage) of simulation points in sample locations which have correctly been conditioned to the input data. As mentioned before, the conditioning-test results were quite sound except that they were weaker for the TSIM methods especially with more quenching steps (almost $89.5 \%$ to $95.22 \%$ for the TSIM methods and above $99 \%$ for SISIM method).

In order to check the histogram-reproduction of the simulations, two methods of deviation rates framework and the chi-square test of the homogeneity of populations were proposed and applied here. Some variants of these two methods have already been employed in several other applications and statistical methodologies with differences in formulation details and applications. For instance, the first framework contains a very similar criterion to the absolute percent error (APE) known also as absolute percent deviation (APD) and its other variant, mean absolute percent error/deviation (MAPE/MAPD) criterion ("Mean Absolute Percentage Error." Wikipedia. Web. Accessed: Feb. 2012). Such criteria have been widely applied in time-series analyses (e.g. Yaffee and McGee 2000, p.17 and "Time-series Forecasting Error Statistics." Time-series Forecasting Error Statistics Web. Accessed: Feb. 2012) due to their simplicity and straightforward relation to the target values. However, in the first framework proposed in this study by the author termed here as the deviation rates framework, some special steps and conditions, as well as some extra criteria, beside the APE or MAPE criterion, have been considered with specific steps to target the goals of proportions-reproduction check of subsurface geostatistical simulations more adequately. In this method, the proportions of soil categories inferred from the borehole data (with necessary corrections to make them representative) constructed the reference distribution. Then, the rate of deviation for the proportions of each soil category from its reference value for each realization was calculated. The soil classes with the highest proportions in the reference distribution (i.e. the biggest classes) were regarded as the key categories. Any realization that its deviation rates for these key categories (from their reference values) were greater than an acceptable deviation rate was rejected. The advantage of using this routine is not only for having a straighter link to the proportion values but also for its practical simplicity. In addition and more importantly, this method puts more emphasize on the bigger classes which are statistically more reliable and at the same time, it forces the proportions of the smaller classes to become volumetrically closer (relative to the whole volume of the model) to those of the reality. The deviation rates framework proposed here contains a 
similar criterion to the absolute percentage error (APE) applied in some geostatistical literature (e.g. He et al. 2009, p. 627) but with additional considerations and improvements which has been explained in part (2)a (Section 5.3.3, Chapter 5). To the knowledge of the author, this proposed framework has not yet been used in geostatistical simulation problems or at least the author is not aware of the existence of such a framework.

The second proportions-reproduction test which has been applied here (i.e. the chi-square test of homogeneity of populations) is being used to evaluate the homogeneity of population tests which indicate whether or not two samples come from the same populations. However, to the extent of the author's knowledge and search, this criterion has not been employed in geostatistical applications yet. Therefore, the author suggests the insight of homogeneity test for evaluating the acceptability of proportionsreproduction of a simulation in geostatistical simulations of the categorical variables especially that it does not consider any of the available distributions as the reference distribution.

The next step was to assess the variograms-reproduction of the geostatistical realizations selected from the previous stages. To test the general variograms-reproduction of all realizations of a geostatistical simulation method, a graphical routine was applied. In this method, for each soil category in vertical and horizontal directions, the model indicator variograms as well as experimental indicator variograms of all the realizations of a simulation method were plotted in the same graph. Then, the general behavior of experimental indicator variograms and their corresponding model was compared to each other. A similar approach was applied for the TPs-reproduction test. In addition, the Julian Ortiz's proposed analytical method (Ortiz 2007) for variograms-reproduction test together with the reasons believed here for its inappropriateness for such applications has been discussed. Yet, some more suitable analytical methods as well as necessary computer codes could be suggested to be developed for similar problems.

The geological soundness evaluations were also conducted in this study to evaluate how reliable the generated simulations are. Still, no considerable contradictions to the expected geologies have been found in this case though this evaluation was considerably qualitative than quantitative.

Besides the evaluation of the realizations generated by these simulation methods, the simulation methods themselves could also be compared to each other to imply and suggest the best simulation algorithm(s). This comparison of different simulation 
algorithms was also conducted in this study. Again, based on the criteria similar to those used in selecting the best realizations of simulation methods as well as some other ones, the TP/MC (TSIM simulation method) was decided to be much better than the SISIM method while the biggest disadvantage of the TP/MC algorithm (i.e. its rather big runtime demand) was still there. The algorithms with more number of realizations which represented statistical/geostatistical and geological acceptability or closer statistics/geostatistics and geology to that of the expectation were suggested to be more efficient. The details of these criteria and evaluation of the efficiencies of these simulation methods have been explained in Chapter 6 .

\subsection{Some suggestions, comparisons, and conclusions inferred from this study}

Investigations conducted in this study for the proportions-reproduction, suggested that the simulations produced by the TP/MC (TSIM) method had considerably more acceptable quality than those of the SISIM method. This judgment were based on the (geo)statistical and geological criterions mentioned in the previous section. Hence, the two explained frameworks for proportions-reproduction evaluations could be recommended as key tests for evaluating the geostatistical simulations and selecting the best ones. For the TPs- and variograms-reproduction evaluations, more appropriate criteria and computer codes could be developed. In addition, it can be suggested that the mentioned framework can be applied in the similar cases of modeling the facies or other categorical attributes in subsurface and for the similar cases of the geotechnical models.

The TPs- and variograms- reproduction analyses in the present study have confirmed the acceptability of the reproduction of the spatial variability structures of all selected realizations from the previous stages. In other words, the TP/MC realizations which were evaluated to be acceptable in terms of conditioning to the input data as well as proportions-reproduction represented also acceptable TPs- and variograms-reproduction.

Although the other available algorithms for simulation of categorical variables such as multiple-point statistics (MPS), truncated pluri-Gaussian simulation (TPGS) method, object-based simulation method, and so on, have not been applied in this study, however, the results from other literature as well as logical reasoning which has been discussed in the previous section, have suggested the TP/MC method as one of the best solutions for 
the applications similar to this study. This comparison should especially be defensible, since, no training images or a speculation of the transition patterns among soil categories or any special information about how the geological objects may look like, were available in this study to make it suitable to use the other simulation methods. It seems that the application of TP/MC method, with the suggested framework here, in similar cases should noticeably be suitable so that its application could be suggested for similar cases too.

There were several (while still not so many) literature about the application of TP/MC (i.e. TSIM or T-PROGS) method in three-dimensional modeling of subsurface categorical attributes discussing the suitability of TP/MC and SISIM methods and their comparison to each other or to the other geostatistical simulation methods (e.g. Weissman et al. 2002, Maji et al. 2006, Weissman et al. 1999, and Schornberg et al. 2010, dell'Arciprete et al. 2012).

In fact, the results of the present dissertation as well as those of some others from the literature (e.g. Weissman et al. 2002, Maji et al. 2006, Weissman et al. 1999, and Schornberg et al. 2010) have demonstrated a sizeable efficiency of the TP/MC method in producing sound geostatistical simulations (apart from its rather long running time) especially over the SISIM technique. For that reason, some attempts also were made here to improve the TSIM algorithm by reducing the computational time, enhancing the simulation quality, and/or adding more data integration capacity to the method. Still, further researches are required to accomplish the improvements of this algorithm while a notable improvement was not achieved in this study yet especially due to the limited time of this research. However, these attempts did not reach a deadlock but the research time limits, hindered further efforts.

Moreover, some suggestions could be made to evaluate the effect of combining TSIM with other geostatistical simulation techniques such as multiple-point geostatistical simulation methods.

However, because in the TP/MC method, the modeling of the transitions from any category to another one in each distance has direction, it can be expected that this method would work more efficiently than the other two-point geostatistical techniques as this research and the similar literature (e.g. Weissman et al. 2002, Maji et al. 2006, Weissman et al. 1999, and Schornberg et al. 2010) also suggest. Yet, as another suggestion, the efficiency of the multiple-point geostatistical (MPS) methods for similar problems can be tested and compared to that of the TP/MC method (e.g. like dell'Arciprete 2012), or at 
least the produced results out of the TP/MC method could be evaluated using the MPS methods (e.g. Boisvert, J B et al. 2010). A clearer suggestion in combination of the TP/MC with MPS method could be the application of MPS metrics (e.g. Boisvert, J B et al. 2010) either in the simulated annealing stage of the TP/MC method in simulation optimization or the selection of the best realizations produced by the TP/MC method. These checks can be especially more interesting for modeling the curvilinear, long-range, and complex geological patterns. However, the MPS methods entail some shortcomings and difficulties that could make hinders for easily using them in similar applications. For instance, the necessity of having the training images (TIs) besides their probable big influence on the simulation results rather than considering the effect of real underlying structures (e.g. Ortiz 2004) could be pointed out from these shortcomings. The growing use of the MPS methods in the petroleum applications due to their usually limited available data for estimation and simulation is of the reasons for using training images and the MPS methods suitability. However, there are typically more available data in the mining applications or comparable cases like the geotechnical subsurface modeling where plenty of local data could be present.

Another alternative to the SISIM and TP/MC algorithms for the facies and categorical data simulation is Truncated Gaussian simulation (TGS) method which has frequently been applied in facies modeling of reservoirs especially for hydrocarbon modeling. In the truncated Gaussian simulation method, the realizations of a continuous Gaussian variable is generated and truncated in series of thresholds to produce the categorical variables in simulations. This method can work sensibly when a clear ordering in the facies sequence is expected (Deutsch 2002, p. 204). Then the categorical variable can be seen as a continuous Gaussian variable that is truncated in specific thresholds (Deutsch 2002, p. 204). The produced patterns form this method would represent much better connectivity than those produced by the methods based on Gaussian fields such as SISIM, while the ordering in the facies occurrence would be kept. In addition, the spatial structure for the variability of the facies reflected by their indicator variograms would also be reproduced adequately. However, the TGS method has some restrictions such as its inapplicability for the cases where a clear ordering in the occurrences of categories is not expected. For instance, in this study, there were many (horizontally or vertically) adjacent soil class 3 samples found next to the soil class 1 which means that there were no ordering in the transitions of the categories in this data-set. In addition, in the TGS method, modeling different anisotropies for different categories (i.e. each soil class here) is not possible. The 
truncated pluri-Gaussian (TPGS) simulation is an extension of the TGS method which makes the reproduction of the geologically more complex transitions feasible among the various classes. It makes use of more than one Gaussian random variable. Then, it truncates these Gaussian random variables in specific thresholds to obtain the simulation categories. The TPGS method enjoys some advantages such as resolving short-scalevariations problem, a good reproduction of proportions and indicator variograms or crossvariograms (Galli et al. 1994, p. 217; Cáceres et al. 2010, p. 2; Loc'h and Galli 1997; Deutsch 2002, pp. 204-205), and the capability of including some geological interpretations for example about the possible transitions among different categories or locally varying proportions (Loc'h and Galli 1997). However, this method still suffers from some drawbacks. For instance, it is not easy to define the simulation model parameters, such as thresholds or transition rules, etc., to obtain the expected geological features. Another option could be the application of cleaning cell-based facies realizations. This method not only reduces the effect of short-scale variations of the classes pattern produced by the traditional simulation methods, it can also maintain the proportions-reproduction of soil classes in the simulations (Deutsch 2002, p. 210). One of the solutions to perform cleaning cell-based facies of realizations is based on the dilation and erosion scheme. This solution is suited for cleaning images including only two facies although a nested approach can be implemented to produce a more complicated simulation (Deutsch 2002, p. 210). Some other Markov chain simulation methods such as the coupled Markov chain (CMC) simulation method (e.g. Elfeki and Dekking (2007); the Markov chain geostatistics (MCG) by Li and Zhang (2010), or Li et al. (2005)) have been proposed to simulate the facies in the models. However, they are not still sufficiently developed and suited for three-dimensional modeling, while they sometimes can produce artefacts in the simulations. Some more details especially about the practical side of these methods have been summarized in Table 1.3.

Further investigations can still be made to improve and test the available categoricalvariable simulation methods and compare them to each other.

\subsection{Suggestions for further research}

Based on the suggested evaluation criteria, the results of the TP/MC simulations of the soil facies, in the case of Göttingen test site, have presented sizably more sound results 
compared to those of the SISIM method. Hence, the application of the TP/MC method about the other similar cases is suggested and speculated to offer considerably enhanced quality of simulations compared to those of the SISIM and traditional methods and sensible simulated patterns in general. This claim can be supported by theoretical discussions and practical findings mentioned in literature (e.g. Carle and Fogg 1996; Carle and Fogg 1997; Si-Yong Lee et al. 2007, Carle et al. 1998, Schornberg et al. 2010; Weissman et al. 1999; Ritzi 2007).

As discussed before, the transition-probability Markov chain simulation method improved the histogram reproduction, spatial variability structure reproduction (evaluated here by indicator variograms and transition-probabilities), the connectivity and structure of the sedimentary geo-bodies and hence the geological soundness (mostly qualitatively). These TSIM methods are also more capable of integrating information in the model such as the length statistics of the geological bodies. Therefore, the application of the TSIM methods could be suggested for similar problems. However, they still face some important drawbacks such as the long computational time and more practical and theoretical complexity. Pursuing the attempts pointed out in Chapter 5 (Section 5.5) is suggested also here to improve the TSIM algorithm to achieve a more high-quality simulations as well as a faster algorithm with a capability of more data integration. In general, some goals, such as the following points, could be defined for further researches and improvements of the transition-probability Markov chain technique:

(a) Reducing the computational time and practical complexity of the algorithms.

(b) Adding the capacity of integrating more information in the simulation models.

(c) Making automations on the most of the simulation and models-validation steps in the framework of a software wizard and guided steps; e.g. finding the best parameters for estimations and simulations, testing the conditioning on the input data, histogram-reproduction assessments, checking the transition-probabilitiesand variograms-reproduction of the realizations, integrating the length statistics in the simulation models more directly, integrating more geological information in the simulation models, suggesting the best realizations and assessing the quality of the realizations in terms of the necessary geostatistical, statistical and geological factors, and so on.

(d) Evaluating the capability of combing the transition-probability Markov chain simulation models with the other geostatistical techniques such as multiple-point 
geostatistics, artificial neural networks, and so on. For instance Boisvert et al. 201 suggested multiple point metrics as other minimum criteria to be to accept a geostatistical realization. (Bohling and Dubios 2003; Ortiz and Deutsch 2004). In other words, a clearer suggestion in combination of the TP/MC with MPS method could be the application of MPS metrics (e.g. Boisvert, J B et al. 2010) either in the simulated annealing stage of the TP/MC method in simulation optimization or the selection of the best realizations produced by the TP/MC method. These checks can be especially more interesting for modeling the curvilinear, long-range, and complex geological patterns. However, the MPS methods entail some shortcomings and difficulties that could make hinders for easily using them in similar applications (e.g. Boisvert, J B et al. 2010). The reason behind suggesting the combination of the multiple-point (MPS) geostatistics with the TP/MC method is that the TSIM algorithm still relies on a two-point statistic (TP) that might be incapable of wholly capturing the spatial structure of the geological complexities, especially for modeling too sophisticated patterns.

(e) In the cases of the existence of analogues, complementary and auxiliary information could be taken into account to improve the models such as Markov chain models of transition-probabilities.

(f) The application of a more general analytical method similar to what have been applied to evaluate the proportions-reproduction of the simulations (e.g. like the homogeneity tests or deviation-rates framework (proposed here by the author)) could be suggested for the variograms- or TPs-reproduction checks in similar applications.

Yet, as another suggestion, the efficiency of using some other geostatistical facies simulation methods such as multiple-point geostatistics (MPS) for similar problems can be tested and compared to that of the TP/MC method.

Moreover, some suggestions could be made to assess the effect of combining TSIM with other geostatistical simulation algorithms such as multiple-point geostatistical simulation methods.

Another suggestion could be the evaluation of the effects of using such simulations in different applications such as flow simulations, temperature or geotechnical parameters to 
evaluate the outcomes of these methods using the results of such simulations and assess which one could be appropriate with which application.

Due to the normal limitations during a $\mathrm{PhD}$ period, the cited suggestions could not be followed at the moment and can be pursued later. 


\section{Acknowledgment}

This $\mathrm{PhD}$ research, either accomplished particularly well or fair, could not be achieved excepting the presence of the chain of several positive factors in spite of numerous hinders, confusions, tough and even disappointing moments. $\mathrm{A} \mathrm{PhD} \mathrm{period,} \mathrm{in} \mathrm{nature,} \mathrm{is} \mathrm{a}$ challenging but sweet procedure of practicing the reaching and crossing the boarders of the existing knowledge. $\mathrm{A} \mathrm{PhD}$ is not only a scientific procedure, but it is also a life going on beside our personal lives or even a big piece of it. When I am looking back, I see countless kindnesses and favors and guidance, scientific and emotional supports from many generous people around me. So, I am heartily and sincerely thankful from all of them for all of their favors and kindnesses towards me. Undoubtedly, after the mighty god, I should thank first of all, my homeland where I was born, grown up, and breathed, for giving me the opportunity to play a role, even so small, in its future. It would be almost impossible for me to start a $\mathrm{PhD}$ except with a financial support and fortunately that was mainly provided by a full scholarship from the Ministry of Science, Research and Technology of the Islamic Republic of Iran and I appreciate and thank it very much. Most especially, I thank my patient and understanding supervisors, Professor Dr. Ingenieur Thomas Ptak and Professor Dr. Martin Sauter for their various kind supports, including the scientific advices, and the facilities, official, and some financial aids. I will never forget their cooperation, patience, and understanding during my research. I can not also forget the kind help and partial financial aid from "Studium International" in the last seven months that reduced my financial concerns to concentrate more on my dissertation. In addition, I am so grateful to my nice colleagues in the Applied Geology department of the Georg-August Unviersity of Göttingen especially to the Göttingen modeling project colleagues; Dr. Torsten Lange, and Dr. Bianca Wagner and the other colleagues including Dr. Till Heinrichs, Dr. Chicgoua Noubactep, and PD Dr. Ekkehard Holzbecher.

I also appreciate the supports of my colleagues in Sahand University of Technology (Tabriz, Iran), especially the Faculty of Mining engineering especially, Associate Professor Dr. Hamid Aghababaei, Associate Professor Dr. Mohammad Jafar Mohammadzadeh Fakhri, Assistant Professor Dr. Parviz Pourghahramani. Iranian students' scientific representatives in Schengen area Dr. Farhad Rahmati, Dr. Mohammad Hossein Abdollahi, and. Dr. M. R. Meshkatoddini had a great share in my work by their understanding guidance and helps. 
Lots of friends outside our faculty also devoted their considerable and open-handed helps and assists, above all, Dr. Iraj Gholami in the faculty of Physics of the University of Göttingen, Dr. Raimon Tolosana Delgado from department of sedimentology of the University of Göttingen and the applied Mathematics of the University of Girona, Spain, and Professor Dr. Ingenieur Uwe Haberlandt from the Institute of Water Resources Management of the University of Hannover. Moreover, I am deeply thankful from many people who generously helped and guided me via email, above all, Associate Professor Dr. Gary Weissman (University of New Mexico) and other people who kindly helped me by email especially Professor Georg Christakos, Dr. Steven F. Carle, Dr. Weidong Li, Dr. Xavier Emery, Dr. Julian Ortiz, Professor Robert Ritzi (Wright State University, USA), Engineer Behrang Koushavand (University of Alberta), Professor Dr. Helmut Schaeben (Technical university of Freiberg), Professor Dr. Jef Caers (Center for Reservoir Forecasting, Stanford University).

Definitely, the friends who gave me the courage to stay determined and motivated always especially in my gloomy and disappointing moments in my personal life and $\mathrm{PhD}$ time especially Dr. Reza Khorasani, Dr. Seyed Habib Ebrahimnejhad Rahbari, Dr. Tobias Geyer, Dr. William Alkhoury, Dr. Hassan Sayyari , Dr. Bijan Nekouei, and some others whom I might have forgotten to name them here, share in any achievement in my $\mathrm{PhD}$. Also, I appreciate the supports and advice of my nice friends Dr. Saber Qanbari, Dr. Alireza Moshki, Dr. Mohsen Alizadeh, Mr. Seyed Zeynalabedin Hosseini, Mr. Ammar Rafeie Emam during my research and emotionally supporting me.

The understanding former scientific representatives of Iranian students in Europe; Dr. Farhad Rahmati and Dr. Mohammad Hossein Abdollahi and our present scientific representative, Dr. Mohammad Reza Meshkatoddini, and my appreciative colleague in the Sahand University of Technology Dr. Esmaeil Najafi Aghdam who was doing his $\mathrm{PhD}$ in Paris some years ago and did me a lot of favors in sorting-out the official hinders, and kind helps of Professor Dr. Mohammad Ali Keynejhad (the founder of Sahand University of Technology), Associate Professor Dr. Mohammad Reza Chenaghlou, Professor Dr. Farzan Ghalichi, the presidents of Sahand University of Technology who compassionately supported me from the beginning of the way.

Very importantly, all the kindnesses, patience, and helps of my beloved parents Tayebeh Sanatgar Jeddi and Esmaeil Ranjineh Khojasteh, and my brothers; Khalilollah, and Engineer Ataollah can never be forgotten. Especially, I thank my understanding brother, 
Engineer Ata. Ranjineh Khojasteh who also, as an expert, provided me several resources and opinions related to the geotechnical issues.

I should sincerely and specially thank Mr. Sadegh Nasiri Alqalandis, Mrs. Robabeh Nasiri Alqalandis, and Mrs. Nasrin Soleymani Shiraz who assisted me devotedly in the official affairs of getting my scholarship from the Ministry of science in Iran.

I heartily appreciate all of these people and whom I forgot to name them and again and most especially, I thank again my kind advisers Professor Dr. Ingenieur Thomas Ptak, and Professor Dr. Martin Sauter. 


\section{List of important acronyms, abbreviations, and initialisms}

BME

Bayesian maximum entropy method for geostatistical simulation

CCDF conditional cumulative density (= distribution) function

CMC coupled Markov chain geostatistical simulation method

DR or D.R. deviation rate of a proportion

IK. indicator kriging geostatistical estimation technique

MCMOD. an algorithm and program of the TPROGS software for transition probabilities modeling using Markov chain models MC Markov chain

MPS multiple-point statistics method for geostatistical simulation

SGS. sequential Gaussian simulation

SISIM sequential indicator (geostatistical) simulation method

SRSD. sum of the rates of squared deviations

TGS. Truncated

Gaussian simulation.

TSIM. .the geostatistical simulation algorithm and program of the TP/MC method

TP Transition probability between two categories

TP/MC transition-probability Markov chain geostatistical simulation method 
TPC triple-Markov chain geostatistical simulation method

T-PROGS or TPROGS The computer software package of TP/MC method in the FORTRAN programming language.

Q-Q plot quantile-quantile plot to compare two distributions

$S$ the entropy of bed-to-bed transition frequencies

$\pi_{i j}$ embedded transition probability from category $i$ to category $\mathrm{j}$

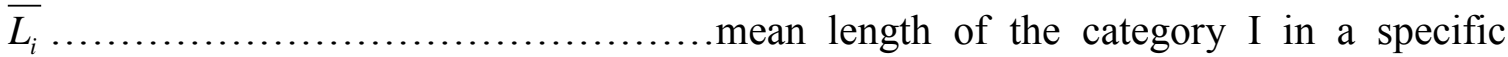
direction

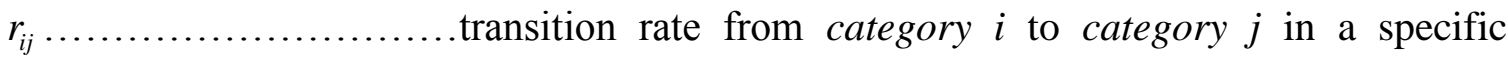
direction $t_{i j}(h)$ transition probability from category $i$ to category $j$ along a separation vector of $h$ in a specific direction

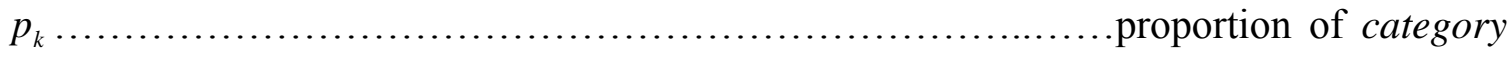
$k$ $\chi^{2}$ chi-square (or chi-squared) statistic

$\gamma^{*}(h)$ sample (=experimental) variogram of regionalized variable along a separation vector of $h$ $\gamma(h)$ .model variogram of regionalized variable along a separation vector of $h$ 


\section{Bibliography}

"Advanced Mapping of Environmental Data: Geostatistics, Machine Learning and Bayesian Maximum Entropy." - Wiley Online Library. N.p., n.d. Web. 23 May 2012. <http://onlinelibrary.wiley.com/book/10.1002/9780470611463>.

Aghajani, H . Farshbaf, and H . Soltani Jigheh. "Reduction of Landslide Risk in Substituting Road of Germi-Chay Dam." Geotechnical Risk and Safety: Proceedings of the 2nd International Symposium on Geotechnical Safety and Risk, Gifu, Japan, 11-12 June 2009. Ed. Yusuke Honjo, Takashi Hara, Makoto Suzuki, and Feng Zhang. Boca Raton: CRC, 2009. N. pag. Print. Print ISBN: 978-0-41549874-6, eBook ISBN: 978-0-203-86731-0, DOI: 10.1201/9780203867310.ch45.

Armstrong, M. ,. Basic Linear Geostatistics. Berlin: Springer, 1998. Print.

Arp, G., Hoffmann, V.-E. S. Seppelt, and W. Riegel. "Exkursion 6: Trias Und Jura Von Göttingen Und Umgebung." Proc. of 74. Jahrestagung Der Paläontologischen Gesellschaft, Göttingen. N.p.: n.p., Oktober 2004. 147-92. Print. Originally cited in Wagner et al. (2007).

"Bearing Capacity." Wikipedia. Wikimedia Foundation, 05 Apr. 2012. Web. Apr. 2012. <http://en.wikipedia.org/wiki/Bearing_capacity>.

Bennett V, George L., Gary S. Weissmann, Gregory S. Baker, and David W. Hyndman. "Regional-scale Assessment of a Sequence-bounding Paleosol on Fluvial Fans Using Ground-penetrating Radar, Eastern San Joaquin Valley, California." Geological Society of America Bulletin 118.5 (2006): 724-32. Print.

Bierkens, Marc F.P., and Henk J.T. Weerts. "Application of Indicator Simulation to Modelling the Lithological Properties of a Complex Confining Layer." Geoderma 62.1-3 (1994): 265-84. Print. Online 10.1016/0016-7061(94)90040-X 
Bierkens, Marc F.P., and Henk J.T. Weerts. "Application of Indicator Simulation to Modelling the Lithological Properties of a Complex Confining Layer." Geoderma 62.1-3 (1994): 265-84. Print. Online DOI: 10.1016/0016-7061(94)90040-X

Bohling, G. C., and M. K. Dubois. An Integrated Application of Neural Network and Markov Chain Techniques to Prediction of Lithofacies from Well Logs. Rep. no. 2003-50. KGS (Kansas Geological Survey) -- Energy Resources -- Open-file Reports, June 2005. Web. 2008-2009.

<http://www.kgs.ku.edu/PRS/publication/2003/ofr2003-50.pdf>.

Boisvert, Jeff B., Michael J. Pyrcz, and Clayton V. Deutsch. "Multiple Point Metrics to Assess Categorical Variable Models." Natural Resources Research 19.3 (2010): 165-75. Print.

Caers, J. "Adding Local Accuracy to Direct Sequential Simulation." Math. Geosciences 32.7 (2000): 815-50. Web. DOI: 10.1023/A:1007596423578

Caers, J., and T. Zhang. "Multiple-point Geostatistics: A Quantitative Vehicle for Integrating Geologic Analogs into Multiple Reservoir Models." Integration of Outcrop and Modern Analog Data in Reservoir Models. By G. M. Grammar. Ed. P. M. Harris and G. P. Eberli. Vol. 80. N.p.: AAPG, 2004. 383-94. Print.

Caers, Jef. Petroleum Geostatistics. Richardson, TX: Society of Petroleum Engineers, 2005. Print.

Caers, J.k., S. Srinivasan, and A.g. Journel. "Geostatistical Quantification of Geological Information for a Fluvial-Type North Sea Reservoir." SPE Reservoir Evaluation \& Engineering 3.5 (2000): 457-67. Print.

Carle, S. F., and G. E. Fogg. "Modeling Spatial Variability with One- and Multidimensional Continuous Markov Chains." Math. Geosciences 29.7 (1997): 891917. Print. online DOI: 10.1007/BF02083656 
Carle, S. F., E. M. Labolle, G. S. Weissmann, and D. V. Brocklin. "Conditional Simulation of Hydrofacies Architecture: A Transition Probability/Markov Approach, in Hydrogeologic Models of Sedimentary Aquifers." Hydrogeologic Models of Sedimentary Aquifers. By Gordon S. Fraser and J. Matthew Davis. Vol. 1. Tulsa, OK: SEPM (Society for Sedimetary Geology), 1998. 147-70. Print. Ser. 1.

Carle, S. F. "Use of a Transition Probability/Markov Approach Geostatistical Simulation of Facies Architecture." Applied Reservoir Characterization Using Geostatistics. Proc. of American Association of Petroleum Geologists (AAPG) Hedberg Symposium, The Woodlands, TX, USA. Geosciences and Environmental Technologies Division, Lawrence Livermore National Laboratory, Livermore, California 94551, 16 Aug. 2008. Web. 2009. <ftp://geologyftp.gg.uwyo.edu/YeZhang/Geosta_Readings/Enviromental/Transitio nProbability.pdf $>$.

Carle, Steven F., and Graham E. Fogg. "Transition Probability-based Indicator Geostatistics." Mathematical Geosciences 28.4 (1996): 453-76. Print.

Carle, Steven F. Carle, Steven F. "T-PROGS: Transition probability geostatistical software." University of California, Davis, CA (1999). Web. 2007. <http://gmsdocs.aquaveo.com/t-progs.pdf>. Carle, Steven F. "T-PROGS: Transition probability geostatistical software." University of California, Davis, CA (1999).

Chessa, Antonio G. "A Markov Chain Model for Subsurface Characterization: Theory and Applications." Mathematical Geology 38.4 (2006): 503-05. Print.

Chiles, Jean-Paul, and Pierre Delfiner. Geostatistics: Modeling Spatial Uncertainty. New York: Wiley, 1999. Print. 
"Chi-Square Test of Homogeneity." Chi-Square Test of Homogeneity, AP Statistics Tutorial. StatTrek.com, Stat Trek Website, n.d. Web. Summer 2011. <http://stattrek.com/ap-statistics-4/homogeneity.aspx>.

Christakos, George. Modern Spatiotemporal Geostatistics. Oxford: Oxford UP, 2000. Print.

Clausen, Flemming Lund. A Geostatistical Study , South Greenland. Roskilde, Denmark: Risøo National Laboratory, 1982. Print. Online Available: www.risoe.dk/rispubl/reports_INIS/RISOR468.pdf, Web accessed: Web. Aug.Sept. 2012. <http://www.mineweb.com/mineweb/view/mineweb/en/page674?oid=123488>

Dai, Z., R. Ritzi Jr., and D. F. Dominic. "Improving Permeability Semivariograms with Transition Probability Models of Hierarchical Sedimentary Architecture Derived from Outcrop Analog." Water Resources Research 41.W07032 (2005): 1-13. Web. Online doi:10.1029/2004WR003515.

"Danie G. Krige." Wikipedia. Wikimedia Foundation, Mar. 2012. Web. Apr. 2012. <http://en.wikipedia.org/wiki/Danie_G._Krige>.

Das, Braja M. Geotechnical Engineering Handbook. Ft. Lauderdale, FL: J. Ross Pub., 2011. Print.

Davis, Bruce M. "Uses and Abuses of Cross-validation in Geostatistics." Mathematical Geology 19.3 (1987): 241-48. Print.

Davis, John Clements. Statistics and Data Analysis in Geology. New York: Wiley, 2002. Print.

De-fu, Che, Wu Li-xin, and Yin Zuo-ru. "3D Spatial Modeling for Urban Surface and Subsurface Seamless Integration." Geoscience and Remote Sensing Symposium. 
Proc. of Geoscience and Remote Sensing Symposium, 2009 IEEE International, IGARSS 2009, Cape Town. Vol. 3. N.p.: n.p., 12-17 July 2009. III-392-II-395. Web. 2012.

<http://ieeexplore.ieee.org/stamp/stamp.jsp?tp=\&arnumber=5417787\&isnumber= 5417750>. IEEE Xplore digital library, doi: 10.1109/IGARSS.2009.5417787.

Dekking,, M., A. Elfeki, C. Kraaikamp, and J. Bruining. "Multi-Scale and MultiResolution Stochastic Modeling of Subsurface Heterogeneity by Tree-Indexed Markov Chains." Computational Geosciences 5.1 (2001): 47-60. SpringerLink. Springer Science Business Media (Springer Netherlands)/ Kulwer Academic Publishers, Printed in Netherlands, 01 Mar. 2001. Web. 2008-2010. <http://dx.doi.org/10.1023/A:1011610003277, http://www.springerlink.com/content/kkt76458r427530h/>. Online doi: 10.1023/A:1011610003277. ISSN: 1420-0597. dell'Arciprete, Diana, et al. "Comparison of three geostatistical methods for hydrofacies simulation: a test on alluvial sediments." Hydrogeology Journal 20.2 (2012): 299311.

"Deterministic Model." What Is ? Definition and Meaning. BusinessDictionary.com. WebFinance, Inc., n.d. Web. Sept. 2012. <http://www.businessdictionary.com/definition/deterministic-model.html>. Deutsch, Clayton V., and A. G. Journel. GSLIB: Geostatistical Software Library and User's Guide. New York: Oxford UP, 1998. Print.

Deutsch, Clayton V. Geostatistical Reservoir Modeling. Oxford: Oxford UP, 2002. Print. D'Or, D., and P. Bogaert. "Spatial Prediction of Categorical Variables with the Bayesian Maximum Entropy Approach: The Ooypolder Case Study." European Journal of Soil Science 55.4 (2004): 763-75. Print. 
D’Or, D. "Spatial Prediction of Soil Properties: The Bayesian Maximum Entropy Approach." Diss. Université Catholique De Louvain, AGRO/MILA - Département Des Sciences Du Milieu Et De L'aménagement Du Territoire, 2003. DIAL : Spatial Prediction of Soil Properties the Bayesian Maximum Entropy Approach. 2003. Web. 2009-2010. <http://hdl.handle.net/2078/4592>.

Ecker, M. D. "Geostatistics : Past, Present and Future." Encyclopedia of Life Support Systems (EOLSS). Developed under the Auspices of the UNESCO, Eolss Publishers, Oxford ,UK. [http://www.eolss.net] , Accessed from : Mark D. Ecker's Homepage in the Website of the Department of Mathematics, University of Northern Iowa, Cedar Falls, IA, 50614-0506, 15 Oct. 2005 (originally in 2003). Web. Winter 2011. <www.math.uni.edu/ ecker/geostatppf3.doc>.

Elfeki, A. M. M., F. M. Dekking, J. Bruining, and C. Kraaikamp. "Influence of Fine-scale Heterogeneity Patterns on the Large-scale Behaviour of Miscible Transport in Porous Media." Petroleum Geoscience 8.2 (2002): 159-65. Print.

Elfeki, Amro M. M., and F. M. Dekking. "Reducing Geological Uncertainty by Conditioning on Boreholes: The Coupled Markov Chain Approach." Hydrogeology Journal 15.8 (2007): 1439-455. Print.

Elfeki, Amro M.M. "Reducing Concentration Uncertainty Using the Coupled Markov Chain Approach." Journal of Hydrology 317.1-2 (2006): 1-16. Print.

Elfeki, Amro. "Prediction of Contaminant Plumes (shapes, Spatial Moments and Macrodispersion) in Aquifers with Insufficient Geological Information." Journal of Hydraulic Research 44.6 (2006): 841-56. Print.

Emery, Xavier. "Statistical Tests for Validating Geostatistical Simulation Algorithms 约." Computers \& Geosciences 34.11 (2008): 1610-620. Print. 
Fahmy, H. M., and E. A. Elsayed. "Detection of Linear Trends in Process Mean." International Journal of Production Research 44.3 (2006): 487-504. Print.

Falivene, O. A. "Statistical Grid-based Facies Reconstruction and Modeling for Sedimentary Bodies: Alluvial-palustrine and Turbiditic Examples." Geologica Acta 5.3 (2007): 199-230. Print.

Falivene, O. A. Testing Three-dimensional Facies Reconstruction and Modeling Techniques Applied to Cored and Outcropping Analogues. Examples from Swamp Coal Zones to Alluvial Fans and Marine Turbidite Sequences. Diss. University of Barcelona, Spain, 2006. N.p.: n.p., n.d. Print.

Falivene, O., L. Cabrera, and A. Sáez. "Large to Intermediate-scale Aquifer Heterogeneity in Fine-grain Dominated Alluvial Fans (Cenozoic As Pontes Basin, Northwestern Spain): Insight Based on Three-dimensional Geostatistical Reconstruction." Hydrogeology Journal 15.5 (2007): 861-76. Print. Online DOI: $10.1007 / \mathrm{s} 10040-007-0187-8$

FALIVENE, O., L. CABRERA, J. A. MUÑOZ, P. ARBUÉS, O. FERNÁNDEZ, and A. SÁEZ. "Statistical Grid-based Facies Reconstruction and Modelling for Sedimentary Bodies. Alluvial-palustrine and Turbiditic Examples." Geologica Acta 5.3 (2007): 199-230. Geologica Acta. Library of Geology (UB-CSIC) | Faculty of Geology, Martí I Franquès S/n 08028 Barcelona | Spain, 2007. Web. 2008. 〈http://www.geologica-acta.com/pdf/vol0503a01.pdf>.

Farmer, Chris L. "Geological Modeling and Reservoir Simulation." Mathematical Methods and Modelling in Hydrocarbon Exploration and Production. Ed. Armin Iske and Trygve Randen. Berlin: Springer, 2005. 119-202. Print. 
Feyen, L., and J. Caers. "Quantifying Geological Uncertainty for Flow and Transport Modeling in Multi-modal Heterogeneous Formations." Advances in Water Resources 29.6 (2006): 912-29. Print.

Fleckenstein, Jan H., Richard G. Niswonger, and Graham E. Fogg. "River-Aquifer Interactions, Geologic Heterogeneity, and Low-Flow Management." Ground Water 44.6 Understanding (2006): 837-52. Print.

"Geotechnical Engineering." Wikipedia. Wikimedia Foundation, 05 Aug. 2012. Web. 09 May 2012. <http://en.wikipedia.org/wiki/Geotechnical_engineering>.

Goovaerts, P. "Stochastic Simulation of Categorical Variables Using a Classification Algorithm and Simulated Annealing." Mathematical Geology 28.7 (1996): 90921. Print.

Goovaerts, Pierre. Geostatistics for Natural Resources Evaluation. New York: Oxford UP, 1997. Print.

Grüger, E., H. Jordan, D. Meischner, and P. Schlie. "Mittelpleistozäne Warmzeiten in Göttingen, Bohrungen Ottostraße Und Akazienweg." Geologisches Jahrbuch Reihe A.134 (1994): 167-210. Print. originally cited in Wagner et al. 2007.

Gringarten, E., and C. V. Deutsch. "Teacher's Aide Variogram Interpretation and Modeling 1." Mathematical Geology 33.4 (2001): 507-34. Print. ISSN: 08828121 Hamilton, J. J. "Foundations on Swelling or Shrinking Subsoils." Canadian Building Digests CBD-84 (1966): n. pag. CBD-84. Foundations on Swelling or Shrinking Subsoils. National Research Council Canada, 29 July 2005. Web. Dec. 2011. <http://www.nrc-cnrc.gc.ca/eng/ibp/irc/cbd/building-digest-184.html>.

Hattermann, F. F. "Einführung in Die Geostatistik (4)." Lecture. Home Page of Dr. F. Fred Hattermann (Teaching). Potsdam-Institute Für Klimafolgenforschung (Potsdam Inst. For Climate Reseearch), 29 June 2011. Web. Sept.-Oct. 2011. 
$<$ http://www.pik-

potsdam.de/ fred/geostatistik/einf\%FChrung\%20in\%20die\%20geostatistik\%204. pdf $>$.

Hengel, Tomislav. A Practical Guide to Geostatistical Mapping of Environmental Variables. Rep. JRC European Commission Publication/European Soil Portal, 2007. Web. Sept. 2012.

<http://eusoils.jrc.ec.europa.eu/esdb_archive/eusoils_docs/other/eur22904en.pdf〉. 143 pages, ISN 27261.

Hoffmann, Arp G., V.-E. Seppelt, S., and W. Riegel. Trias Und Jura Von Göttingen Und Umgebung. Rep. no. Exkursion 6. N.p.: n.p., Oktober 2004. Print. 74. Jahrestagung der Paläontologischen Gesell-schaft, Göttinge, pp. 147- 192.

Hohn, Michael Edward. Geostatistics and Petroleum Geology. 2nd ed. Dordrecht: Kluwer, 1999. Print. ISBN 978-0-412-75780-8, Nov 30, 1998, 248 pages.

Isaaks, Edward H., and R. Mohan. Srivastava. An Introduction to Applied Geostatistics. New York: Oxford UP, 1989. Print.

Johansson J. "Which Soils Are Liquefaction Susceptible?" Soil Liquefaction Website. Department of Civil Engineering, University of Washington, n.d. Web. 14 Nov. 2011. <http://www.ce.washington.edu/ liquefaction/html/how/susceptible.html>. Jones, Thomas A., and Yuan, Z. Ma. "Teacher's Aide Geologic Characteristics of HoleEffect Variograms Calculated from Lithology-Indicator Variables." Mathematical Geology 33.5 (2001): 615-29. Web.

Journel, André G., and Wenlong Xu. "Posterior Identification of Histograms Conditional to Local Data." Mathematical Geology 26.3 (1994): 323-59. Print. 
Koltermann, Christine E., and Steven M. Gorelick. "Heterogeneity in Sedimentary Deposits: A Review of Structure-imitating, Process-imitating, and Descriptive Approaches." Water Resources Research 32.9 (1996): 2617-658. Print.

Lade, P., and Jerry A. Yamamuro. Physics and Mechanics of Soil Liquefaction:

Proceedings of the International Workshop on the Physics and Mechanics of Soil Liquefaction : Baltimore, Maryland, USA, 10-11 September, 1998. Proceedings of the International Workshop, Baltimore, Maryland, USA. Rotterdam: A.A. Balkema, 1999. Print.

Lade, P. V., and , Yamamuro, J. A. "Physics and Mechanics of Soil Liquefaction." Proc. of Proc. of the Int. Workshop, Baltimore, Maryland, USA, Baltimore. N.p.: n.p., n.d. N. pag. Print.

Lam, L., D.G. Fredlund, and S.L. Barbour. "Transient Seepage Model for Saturatedunsaturated Soil Systems: A Geotechnical Engineering Approach." Canadian Geotechnical Journal 24.4 (1987): 565-80. Print.

Lee, S.-Y., S. F. Carle, and G. E. Fogg. "Geologic Heterogeneity and a Comparison of Two Geostatistical Models: Sequential Gaussian and Transition Probability-based Geostatistical Simulation." Advances in Water Resources 30.9 (2007): 1914-932. Print.

Leeder, M. R. Sedimentology: Process and Product. London [etc.: George Allen \& Unwin, 1982. Print.

Leuangthong, Oy, Jason A. McLennan, and Clayton V. Deutsch. "Minimum Acceptance Criteria for Geostatistical Realizations." Natural Resources Research 13.3 (2004): 131-41. Print.

Li., W., B. Li., Y. Shi, D. Jacques, and J. Feyen. "Effect of Spatial Variation of Textural Layers on Regional Field Water Balance." Water Resources Research 37.5 
(2001): 1209-219. Effect of Spatial Variation of Textural Layers on Regional

Field Water Balance. American Geophysical Union (AGU), May 2001. Web.

2008-2009. <http://www.agu.org/pubs/crossref/2001/2000WR900304.shtml>.

Li, W. "The Nonlinear Markov Chain Geostatistics." Ed. C. Zhang. Geomathematics and GIS Analysis of Resources, Environment and Hazards, Beijing, China, August 2631, 2007 the 12th Conference of the International Association for Mathematical Geology : Proceedings of IAMG 2007. By International Association for Mathematical Geology. Conference, P. Cheng, P. Zhao, and F. Agterberg. Kingston, Ont.: International Association for Mathematical Geology, 2007. 57378. CiteSeerX. 2007. Web. 2009-2010. <http://citeseerx.ist.psu.edu/viewdoc/download;jsessionid=AFEF1610C3AA75E3 98591BE7B2E6D092?doi=10.1.1.94.5095\&rep=rep1\&type $=$ pdf $>$. Accessed from website: http://citeseerx.ist.psu.edu/viewdoc/summary?doi=10.1.1.94.5095

Li, Weidong, and Chuanrong Zhang. "Linear Interpolation and Joint Model Fitting of Experimental Transiograms for Markov Chain Simulation of Categorical Spatial Variables." International Journal of Geographical Information Science 24.6 (2010): 821-39. Print. Online DOI: 10.1080/13658810903127991

Li, Weidong, and Chuanrong Zhang. "A Single-chain-based Multidimensional Markov Chain Model for Subsurface Characterization." Environmental and Ecological Statistics 15.2 (2008): 157-74. Print.

Li, Weidong, and Chuanrong Zhang. "A Single-chain-based Multidimensional Markov Chain Model for Subsurface Characterization." Environmental and Ecological Statistics 15.2 (2008): 157-74. Print. 
Li, Weidong, Chuanrong Zhang, James E. Burt, A.-Xing Zhu, and Jan Feyen. "Twodimensional Markov Chain Simulation of Soil Type Spatial Distribution." Soil Science Society of America Journal 68.5 (2004): 1479-490. Print.

Li, Weidong. "A Fixed-Path Markov Chain Algorithm for Conditional Simulation of Discrete Spatial Variables." Mathematical Geology 39.2 (2007): 159-76. Print.

Li, Weidong. "Markov Chain Random Fields for Estimation Of Categorical Variables." Mathematical Geology 39.3 (2007): 321-35. Print.

"Linking Hierarchical Stratal Architecture to Plume Spreading in a Lagrangian-based Transport Model: 2. Evaluation Using New Data from the Borden Site." Linking Hierarchical Stratal Architecture to Plume Spreading in a Lagrangian-based Transport Model: 2. Evaluation Using New Data from the Borden Site. N.p., n.d. Web. 24 May 2012. <http://www.agu.org/pubs/crossref/2010/2009WR007810.shtml>.

"Liquefaction Potential of Cohesionless Soils Geotechnical Design Procedure." N. Y. State Dep. of Transportation. Ed. Robert A. Burnett. Geotechnical Engineering Bureau, N. Y. State Dep. of Transportation. Engineering Division -Office of Technical Services, Geotechnical Engineering Bureau, Apr. 2007. Web. Oct.-Nov. 2011. <https://www.dot.ny.gov/divisions/engineering/technical-services/technicalservices-repository/GDP-9b.pdf $>$.

Liquefaction Potential of Cohesionless Soils Geotechnical Design Procedure. Rep. Vol. GDP-9. N. Y. State: Geotechnical Engineering Bureau, N. Y. State Dep. of Transportation. Engineering Division -Office of Technical Services, Geotechnical Engineering Bureau, Apr. 2007. Geotechnical Engineering Bureau, Manuals. Web. Fall 2011. <https://www.dot.ny.gov/divisions/engineering/technicalservices/technical-services-repository/GDP-9b.pdf>. 
Marinoni, O. "Improving Geological Models Using a Combined Ordinary-indicator Kriging Approach." Engineering Geology 69.1-2 (2003): 37-45. Print.

Matheron, G. "Principles of Geostatistics." Economic Geology 58.8 (1963): 1246-266. Print.

McKillup, Steve, and M. Darby Dyar. Geostatistics Explained: An Introductory Guide for Earth Scientists. Cambridge, UK: Cambridge UP, 2010. Print.

"Meander (river System Component)." Encyclopedia Britannica Online. Encyclopedia Britannica, n.d. Web. Jan. 2012. <http://www.britannica.com/EBchecked/topic/371575/meander>.

"Meander." Wikipedia. Wikimedia Foundation, Winter 2011. Web. Jan. 2012. <http://en.wikipedia.org/wiki/Meander>.

Meischner, Dieter. Der Leinetal-Graben, Eine Geologische Legende. Populärwissenschaftlicher Vortrag,Öff. Vortrag Am Wissentschafliche Zentrum Der Georg-August-Universität Göttingen, Transcript. Göttingen: n.p., 28.01.2002. Print. Unpubl. Diplomkartierung, Univ. Göttingen, Göttingen, Originally cited in Wagner et al. 2007.

"Model." : Schlumberger Oilfield Glossary. Schlumberger, n.d. Web. 09 Nov. 2011. <http://www.glossary.oilfield.slb.com/Display.cfm?Term=model>.

Myers, D. E. "Redirect Notice." Speech. International Association for Mathematical Geology (IAMG) Dsitinguisched Lecturer. 2008. Redirect Notice. University of Arizona, 2008. Web. Fall 2011. $<$ http://www.google.com/url?q=http://pangea.stanford.edu/ERE/research/scrf/IA MG-StudentChapter/docs/History-of-Geostatistics.ppt>. 
Myers, D. E. "WHAT IS GEOSTATISTICS?" WHAT IS GEOSTATISTICS? Dep. of Mathematics, University of Arizona, Apr. 2002. Web. Winter 2011. <http://math.arizona.edu/ myers/homepage/whatis.html>.

Myers, D. "Geostatistics Glossary." Geostatistics Glossary. Donald Myers’s Home Page, Dept. of Math., University of Arizona, n.d. Web. Sept.-Oct. 2011. <http://math.arizona.edu/ myers/homepage/glossary.html>.

"The Nonlinear Markov Chain Geostatistics." CiteSeerX The Nonlinear Markov Chain Geostatistics. N.p., n.d. Web. 24 May 2012. <http://citeseerx.ist.psu.edu/viewdoc/summary?doi=10.1.1.94.5095>.

Noppé, M. A. "PRACTICAL GEOSTATISTICS FOR ON-SITE ANALYSIS - A COAL EXAMPLE." Proceedings of the Mining Geostatistics Conference. Mining Geostatistics Conference, Kruger National Park, South Africa. Geostatistical Association of South Africa, 1994. Web. 2011. <http://www.snowdengroup.com/Files/GeoStats-4.pdf $>$.

Ortiz, J., and C. V. Deutsch. "Calculation of Uncertainty in the Variogram." Math. Geosciences 34.2 (Feb. 2002): 169-83. Web. Online DOI: 10.1023/A: 1014412218427

Ortiz, Julián M., and Clayton V. Deutsch. "Indicator Simulation Accounting for MultiplePoint Statistics." Mathematical Geosciences 36.5 (2004): 545-65. Print.

Orton, T. G., and R. M. Lark. "Estimating the Local Mean for Bayesian Maximum Entropy by Generalized Least Squares and Maximum Likelihood, and an Application to the Spatial Analysis of a Censored Soil Variable." European Journal of Soil Science 58.1 (2007): 60-73. Print.

Orton, T. G., and R. M. Lark. "Estimating the Local Mean for Bayesian Maximum Entropy by Generalized Least Squares and Maximum Likelihood, and an 
Application to the Spatial Analysis of a Censored Soil Variable." European Journal of Soil Science 58.1 (2007): 60-73. Print.

Park, Eungyu, A. Elfeki, and M. Dekking. "Characterization of Subsurface Heterogeneity: Integration of Soft and Hard Information Using Multi-dimensional Coupled Markov Chain Approach." UIST Conference October 22-25, 2003. Proc. of Underground Injection Science and Technology Symposium, Lawrence Berkeley National Lab., Berkeley, California. U.S. Environmental Protection Agency (OGWDW) and U.S. Department of Energy (FE-NETL and EM/OST), 2005. Web. Accessed: Winter 2011. <http://www.lbl.gov/Conferences/UIST/index.html>.

Park, Eungyu, Amro M. M. Elfeki, Yungoo Song, and Kangjoo Kim. "Generalized Coupled Markov Chain Model for Characterizing Categorical Variables in Soil Mapping." Soil Science Society of America Journal 71.3 (2007): 909-17. Print.

Park, Y.-J., E. A. Sudicky, R. G. McLaren, and J. F. Sykes. "Analysis of Hydraulic and Tracer Response Tests within Moderately Fractured Rock Based on a Transition Probability Geostatistical Approach." Water Resources Research 40.W12404 (2004): 1+. Analysis of Hydraulic and Tracer Response Tests within Moderately Fractured Rock Based on a Transition Probability Geostatistical Approach. American Geophysical Union (AGU), 9 Dec. 2004. Web. 2008-2010. <http://www.agu.org/pubs/crossref/2004/2004WR003188.shtml>. Online doi:10.1029/2004WR003188, 14 PP.

P.E., Gilbert Gedeon. "Bearing Capacity of Soils." Continuing Education and Development, Inc. CED Engineering, Greyridge Farm Court, Stony Point, NY 10980, Oct. 1992. Web. Fall 2011. 
<http://www.cedengineering.com/upload/Bearing\%20Capacity\%20of\%20Soils.pd $\mathrm{f}>$.

Pickard, David K. "Unilateral Markov Fields." Advances in Applied Probability 12.3

(1980): 655-71. Applied Probability Trust. Web. 2011.

<http://www.jstor.org/stable/1426425>. JSTOR.

A Practical Guide to Geostatistical Mapping of Environmental Variables. Rep. N.p., n.d. Web.

R. Khojasteh, E. Geostatistical Investigations of Petrophysical Reservoir Parameters in Nar Gas Field, Booshehr, Iran. Diss. Sahand University of Technology, Tabriz, Iran, Advisor: H. Agheababaei, Summer 2002. Print.

Rubin, Y., I. A. Lunt, and J. S. Bridge. "Spatial Variability in River Sediments and Its Link with River Channel Geometry." Water Resources Research 32.W06D16 (1996): n. pag. Web. 12 PP, Online doi:10.1029/2005WR004853

Sarma, D. D. Geostatistics with Applications in Earth Sciences. 2nd ed. Dordrecht: Springer, 2009. Print. Originally published by Capital Publishing Company, 2002, 2nd ed., 2009, 206 pages

Schlie, P. Hydrogeologie Des Grundwasserwerkes Stegemühle in Göttingen. Selbstverlag Der Geologischen Institute Der Georg-August-Universität Göttingen (internal Publication of the Faculty of Geology, Georg-August-University of Göttingen), Göttingen Department of Paleontology of the Geological Faculty. Vol. 43. Göttingen: n.p., 1989. Print. 143 pages, Figure 32 and Table 28. Internal publication of the Faculty of Geology, Georg-August-University of Göttingen. Originally cited in Wagner et al. 2007.

Schornberg, C., C. Schmidt, E. Kalbus, and J. H. Fleckenstein. "Simulating the Effects of Geologic Heterogeneity and Transient Boundary Conditions on Streambed 
Temperatures — Implications for Temperature-based Water Flux Calculations." Advances in Water Resources 33.11 (2010): 1309-319. Print.

Sinclair, Alastair J., and Garston H. Blackwell. Applied Mineral Inventory Estimation. Cambridge: Cambridge UP, 2002. Print.

Smith, Richard L. "Extreme Value Analysis of Environmental Time Series: An Application to Trend Detection in Ground-Level Ozone." Statistical Science 4.4 (1989): 367-77. Print.

"Soil Liquefaction." Wikipedia. Wikimedia Foundation, 16 May 2012. Web. 21 May 2012. <http://en.wikipedia.org/wiki/Soil_liquefaction>.

"Solifluction." The Free Dictionary. Farlex, 2009. Web. Winter 2011. <http://www.thefreedictionary.com/solifluction>. Originally has been taken from: The American Heritage ${ }^{\circledR}$ Dictionary of the English Language, Fourth Edition copyright $@ 2000$ by Houghton Mifflin Company. Updated in 2009. Published by Houghton Mifflin Company

Stefan, Schorn, and Et. Al. "Mineralienatlas Lexikon - Subrosion." Mineralienatlas Lexikon - Subrosion. Mineralienatlas, 1999-2012. Web. 21 May 2012. <http://www.mineralienatlas.de/lexikon/index.php/Subrosion>.

Stoter, Jantien E., and Peter Van. Oosterom. 3D Cadastre in an International Context: Legal, Organizational, and Technological Aspects. Boca Raton, FL: CRC/Taylor \& Francis, 2006. Print.

Strebelle, S. "New Multiple-point Statistics Simulation Implementation to Reduce Memory and CPU-time Demand." Proceedings to International Association for Mathematical Geology. IAMG 2003, Portsmouth,UK. N.p.: n.p., 2003. N. pag. Print. 
Sun, A. Y., R. W. Ritzi, and D. W. Sims. "Characterization and Modeling of Spatial Variability in a Complex Alluvial Aquifer: Implications on Solute Transport." Water Resources Research Article Number: W04402 44.4 (2008): 1-16. Characterization and Modeling of Spatial Variability in a Complex Alluvial Aquifer: Implications on Solute Transport. American Geophysical Union (AGU), 4 Apr. 2008. Web. 2009. <http://www.agu.org/pubs/crossref/2008/2007WR006119.shtml>.

T., Nix, Wagner B., Lange T., Fritz J., and Sauter M. 3D-Baugrundmodell Der Quartären Sedimente Des Leinetals Bei Göttingen: 3D-subsoil-model of the Quaternary Leinetal Sediments near Göttingen. Proc. of 17. Tagung Für Ingenieurgeologie Und Forum „Junge Ingenieurgeologen“, Zittau, Germany. N.p.: n.p., 2009. Print.

Wagner, B., J. Fritz, J. Nix, and M. Sauter. Ingenieurgeologische Modellierung Im Stadtgebiet Göttingen: 3D Modelling in Engineering Geology within the City of Goettingen. Proc. of 16. Tagung Für Ingenieurgeologie Und Forum,“Junge Ingenieurgeologen, Bochum, Germany. N.p.: n.p., 2007. 267-76. Print.

Wagner, Bianca. Geotechnical Unconsolidated Sediments Classification Scheme Tables for Sedimants with and without Organic Components. Table for the Scheme of the Geotechnical Soils Classification Scheme. N.p.: n.p., November 2009. Print.

Weisstein, E. W. "Eigenvalue." -- from Wolfram MathWorld. A Wolfram Web Resource, n.d. Web. Fall 2011. <http://mathworld.wolfram.com/Eigenvalue.html>.

Wikipedia Contributors. "Mean Absolute Percentage Error." Wikipedia. Wikimedia Foundation, 2012. Web. Mar. 2012.

<http://en.wikipedia.org/wiki/Mean_absolute_percentage_error>. 
Wunderlich, H. G. Zur Abfolge Und Altersstellung Quartärer Bildungen Im Stadtgebiet Von Göttingen Eiszeitalter Und Gegenw. 10. Vol. 10. N.p.: n.p., 1959. Print. pp. 41-55. Figure 5, Table 1; Öhringen. Originally cited in Wagner et al. 2007.

Yamamoto, Jorge Kazuo. "Correcting the Smoothing Effect of Ordinary Kriging Estimates." Mathematical Geology 37.1 (2005): 69-94. Print. online version DOI: $10.1007 / \mathrm{s} 11004-005-8748-7$

Zanon, Stefan, and Oy Leuangthong. "Implementation aspects of sequential simulation." Geostatistics Banff 2004. Springer Netherlands, 2005. 543-548. 


\section{Curriculum Vitae}

\section{Ranjineh Khojasteh, Enayatollah}

Email : enayatkhojasteh@yahoo.com

enayatkhojasteh@gmail.com

\section{Personal information}

First Name Enayatollah (Enayat)

Last Name Ranjineh Khojasteh

Birth date September $19^{\text {th }}, 1977$

Place of Birth Tehran, Iran

Parents Retired teachers.

Marital status Divorced

Hometown Tabriz

Ethnicity Azeri (Azerbayjani of Iran not Azerbayjan Republic).

\section{Educations}

Since October 2006

Ph.D student in Applied Geosciences department of Goettingen University (Research topic: Stochastic/geostatistical three-dimensional modeling of subsurface categorical variables (unconsolidated subsurface zone) and the application of Markov chains for geotechnical/geological characteristics in Goettingen area).

October 2000- September 2002

M.Sc. studies, Mining Engineering-Mineral Exploration, Sahand University of Technology, Tabriz, E. Azarbayjan Province, Iran.

M.Sc. Dissertation topic: Geostatistical estimation and simulation of Geological Parameters in Nar gas field, southern Iran

October 1995/96- July 2000

B.Sc., Mining engineering-exploration, Sahand University of Technology, Tabriz, E. Azarbayjan Province, Iran. B.Sc. Dissertation topic: Geochemical copper exploratory data indexes analysis in Anjerd,Ahar, East Azarbayjan Province, Iran.

1995

High school (secondary school) diploma, Math-Physics in Meshkat high school 


\section{Honors and prizes}

2004/2005

Won the full-scholarship of the Ministry of Science, Research, and Technology of Iran (I.R. of) for doing a $\mathrm{PhD}$ abroad through a highly competitive overall scientific exam.

2003-2005 Very successful teaching rank while giving lectures and managing some Laboratories in Sahand University of Technology.

2002 M.Sc. graduation with first rank in Sahand University of Technology, Faculty of Mining Engineering.

2000 Second rank of country prize in the overall entrance exams in M.Sc.-level of Mining Engineering universities of the entire Iran.

Before 1999 Awarded prizes in several national scientific and cultural competitions such as student mathematics and physics competitions and calligraphy in East Azrabayjan province.

\section{Professional experiences}

Software and computer skills gslib(WinGslib) for geostatistical modeling,T-PROGS for transition-probability-based geostatistical modeling, gOcad for three dimensional modeling and representations, Excel and visual basic macros, Programming (FORTRAN, MATLAB, Pascal, R, Scilab), Surfer, Word, some familiarity with ArcGIS, SAGA GIS, SPSS, etc.

Experiences highlight Geostatistical data analysis for three-dimensional subsurface modeling (especially indicator kriging, indicator simulations, transition-probability-based Markov chain modeling), computer programming, optical and descriptive minerals characterizations...

Since 2001 Three-dimensional stochastic and geostatistical modeling of subsurface.

One case in M.Sc for Nar gas field in southern Iran. During the Ph.D research, for geotechnical and geological facies of Goettingen area.

2000-2004

Consultant engineer in a gemstone exploration and feasibility study, Tabriz, Iran.

Winter 2003

Reserve assessment and estimation workshop for technical supervisors of mines in Orumieh Industries and Mines Office, West Azarbayjan Province, Iran.

Fall 2002- Summer 2005 Lecturer, Sahand University of Technology, Tabriz, Iran.

(Courses taught: exploration drilling, mineralogy, optical mineralogy, petrology)

1998

Geochemical exploration data analysis for copper indices around Anjerd,

Ahar, Tabriz, Iran. 


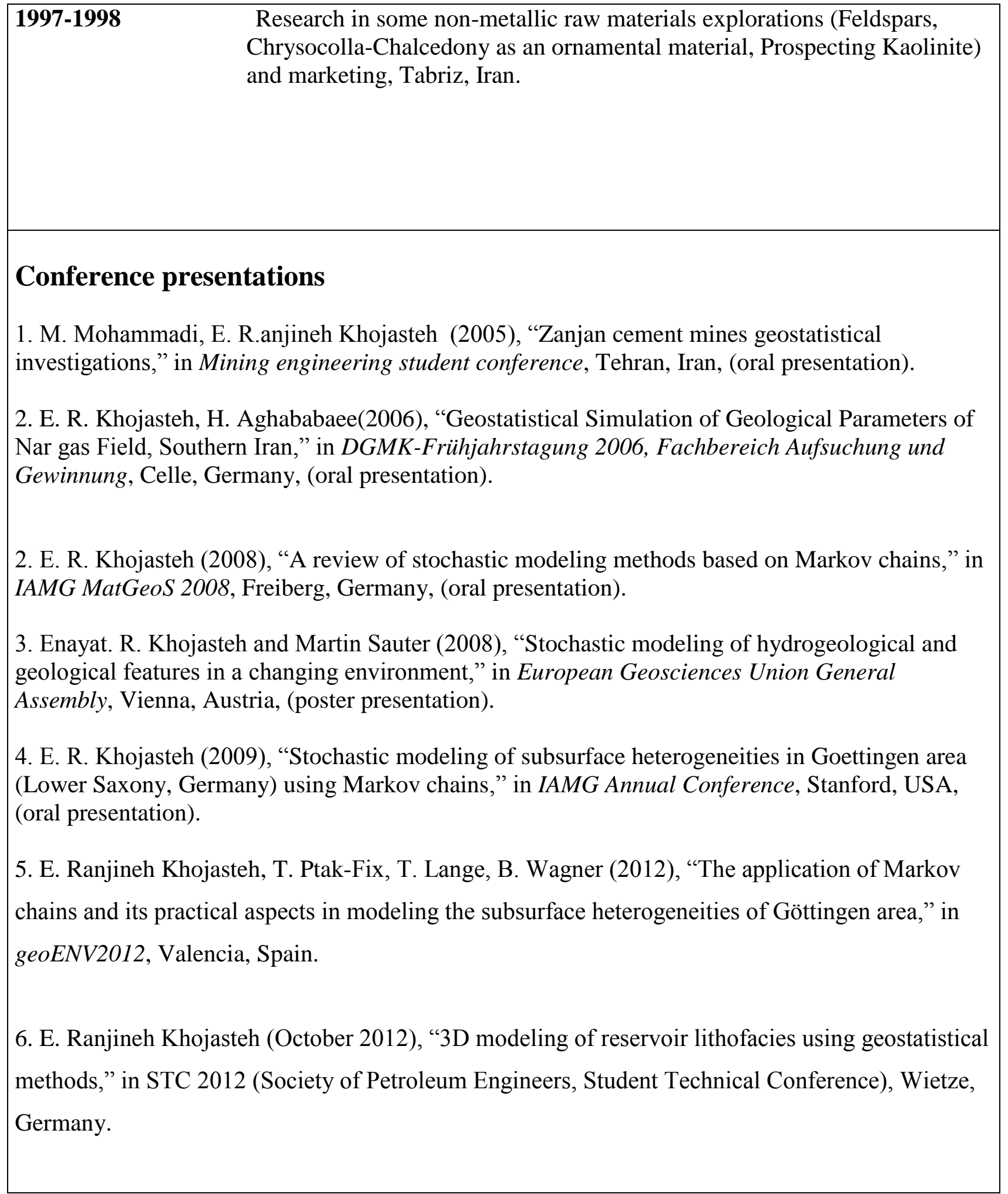




\section{Research and consultation experiences}

Students advising and consulting Zanjan cement project mines geostatistical evaluations, gemstones and faceting research project, ornamental rocks characterization and exploitation research.

Research project E. Ranjineh Khojasteh, Dr. Hamid Aqababaei, Engineer Behrang Koushavand, and colleagues from National Iranian Oil Company (2009), Iranian oil fields three-dimensional geostatistical seismic modeling.

\section{Languages}

English $\quad$ Good $($ IELTS grade $=6.5:$ Listening $=5.5$, Reading $=6.0$, Writing $=7.0$ , Speaking $=7.0$ )

German Low intermediate (last preliminary level including grammar and conversation (Grundstufe 3 and Grundstufe 4)).

Persian $=$ Farsi Fluent $($ Speaking, reading, and writing $)$

Azeri Fluent in speaking and listening comprehension (native), intermediate in writing and reading comprehension.

Other languages: Turkish: intermediate in speaking, reading and lower-intermediate in writing, Arabic: above basic in reading comprehension and listening, basic in speaking. Russian: reading the alphabets and interest to learn.

\section{Hobbies and Interests}

Sports Mount climbing, soccer, cycling, running...

Art Calligraphy, painting, music, etc.

Technical issues Minerals, gems and ores, faceting, electronic circuits and devices, optics, etc.

Other interests Travel, philosophy, history, archaeology, mathematics, languages, etc. 